\title{
3D SOLAR PHOTOVOLTAIC COMMUNITY ENERGY MODELING
}

\author{
by \\ Annie Chow \\ Master of Applied Science, Environmental Applied Science \& Management, Ryerson \\ University, 2012 \\ Honours Bachelor of Science, University of Toronto, 2008 \\ A dissertation presented to Ryerson University \\ in partial fulfillment of the requirements for the degree of \\ Doctor of Philosophy \\ in the program of
}

Environmental Applied Science \& Management

Toronto, Ontario, Canada, 2020

(C) Annie Chow, 2020 


\section{Author's Declaration}

I hereby declare that I am the sole author of this dissertation. This is a true copy of the dissertation, including any required final revisions, as accepted by my examiners.

I authorize Ryerson University to lend this dissertation to other institutions or individuals for the purpose of scholarly research.

I further authorize Ryerson University to reproduce this dissertation by photocopying or by other means, in total or in part, at the request of other institutions or individuals for the purpose of scholarly research.

I understand that my dissertation may be made electronically available to the public. 


\author{
3D Solar Photovoltaic Community Energy Modeling \\ Doctor of Philosophy 2020, Annie Chow \\ Environmental Applied Science \& Management, Ryerson University
}

\title{
Abstract
}

The aim of this research is to increase the assessment ability of solar energy utilization and planning support for clusters of different types of buildings in a mixed-use community. Particular focus will be placed on the analysis of community-based modeling, mapping and forecasting of solar potentials on the rooftops of buildings. New systems and methodologies with appropriate level of detail at a lower computational time are needed to accurately model, estimate and map solar energy potential at a high spatiotemporal resolution. To accomplish this goal and to develop an integrated solution, the assessment ability was investigated using two different types of studies: (1) 3D GIS modeling of a solar energy community, and (2) benchmarking of solar PV radiation software tools. A 3D GIS modeling and mapping approach was developed to assess community solar energy potential. A model was created in ESRI ArcGIS, to efficiently compute and iterate the hourly solar modeling and mapping process over a simulated year. The methodology was tested on a case study area located in southern Ontario, where two different 3D models of the site plan were analyzed. The accuracy of the work depended on the resolution and sky size of the input model. An assessment of solar simulation software tools was performed to evaluate their strengths and weaknesses for performing analysis in the PV modeling process. The software tools assessed were HelioScope, PVsyst, PV*SOL, Archelios, EnergyPlus, and System Advisor Model (SAM). The performance of the software tools were assessed based upon their accuracy in simulation performance against measured data, and the comparison of their physical functions and capabilities. A case study near London, Ontario with an $8.745 \mathrm{kWp}$ PV system installation was selected for analysis, and EnergyPlus was found to have predictions closest to measured data, ranging from $-0.6 \%$ to $3.6 \%$ accuracy. Based upon the GIS study and the evaluation of the six solar software tools, recommendations for the development of a future application to couple GIS with the internal submodels of the software tools were made to create the ideal tool for 3D modeling and mapping of solar PV potential. 


\section{Acknowledgements}

First and foremost, I would like to thank God for blessing me with the strength and courage to finish this dissertation successfully.

I would like to express my sincerest gratitude to my supervisors Dr. Alan S. Fung and Dr. Songnian Li for all the constant support, encouragement, patience and guidance throughout all these years. This dissertation would not have been possible without their vision. I cannot express enough thanks to my committee members Dr. David Naylor and Dr. Phil Walsh for their continued support and helpful advice. Special thanks to my Program Director Dr. Andrew Laursen, for his encouragement and support.

I am extremely grateful to my parents for all their sacrifice, love and support in helping me achieve my dreams. I am very thankful for my sister and best friend Vincy for believing in me and being by my side.

This dissertation would not have been possible without the encouragement and support of my fellow friends and lab mates Pallavi, Anahita, Navid, Danilo, King, Anna, Gulsun, Altamash, Kai, Andrew and Mohammad. You guys have my sincerest gratitude and thanks for always helping and being there for me. Last but not least, I am very thankful for my fat cats Momo and Mimi for sleeping on my desk while I worked. 


\section{Dedication}

This dissertation is dedicated to my beloved grandmother, Chow Yuen Yee. 


\section{TABLE OF CONTENTS}

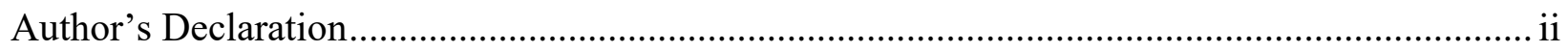

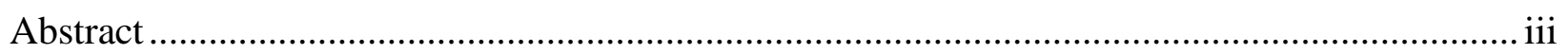

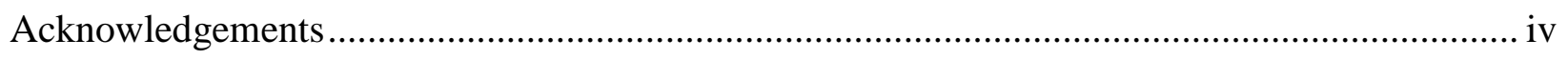

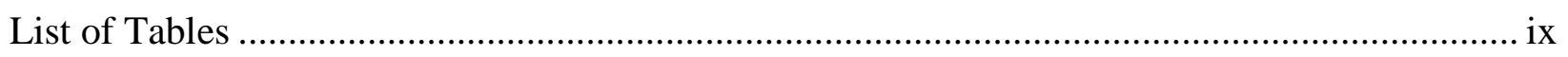

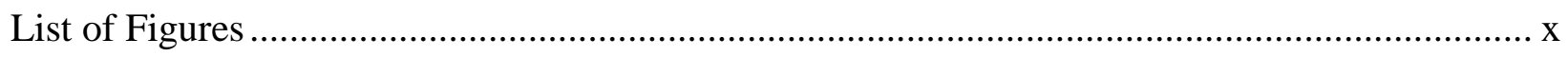

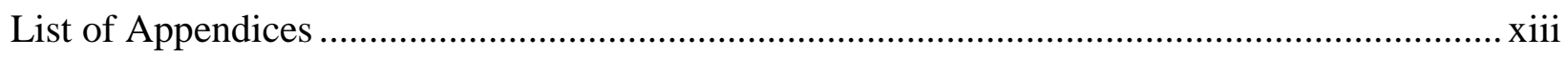

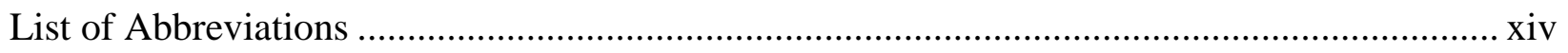

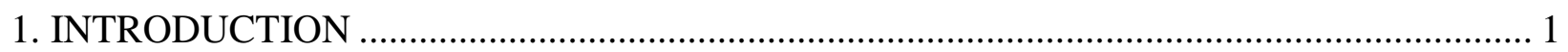

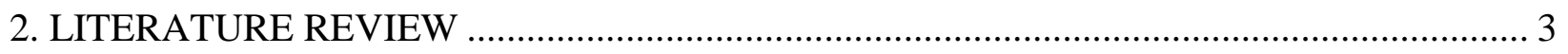

2.1. Solar Photovoltaic Energy ...................................................................................... 3

2.2. Solar Photovoltaic Production Modeling.................................................................... 5

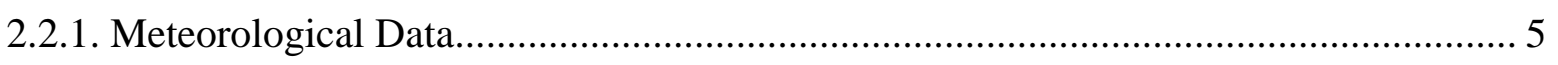

2.2.2. Solar Radiation Models................................................................................... 7

2.2.3. Photovoltaic Performance Models ........................................................................ 13

2.2.4. Inverter Models .............................................................................................. 15

2.2.5. Modeling PV System Losses .............................................................................. 16

2.3. Solar Energy Communities .................................................................................... 17

2.4. Urban Rooftop Solar Energy Modeling ................................................................... 18

2.5. 3D Urban Rooftop and Façade Modeling ................................................................. 20

2.6. Evaluation of Solar Simulation Software Tools ........................................................ 23

2.7. Summary of Solar Software Tools............................................................................. 28

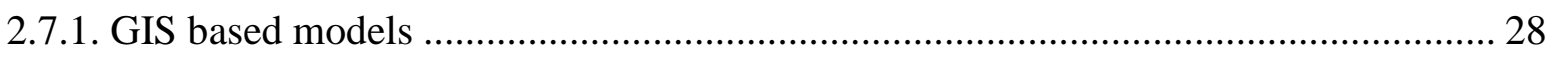

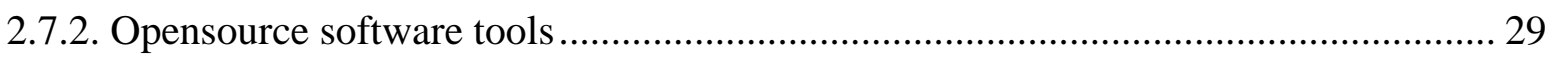

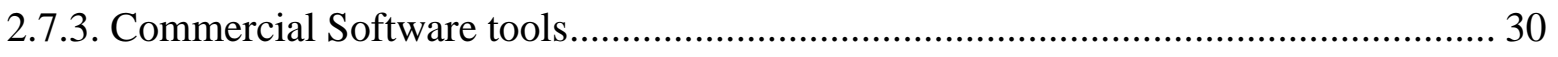

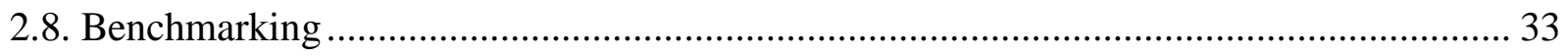

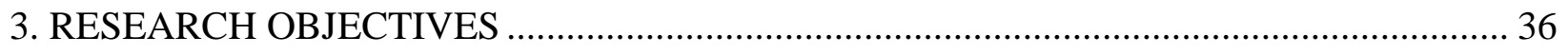

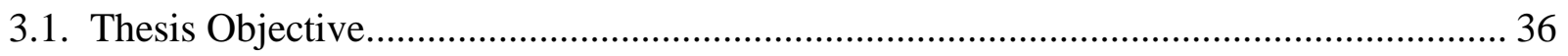

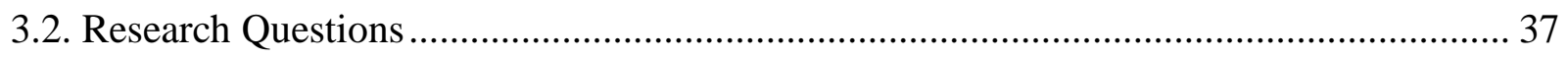

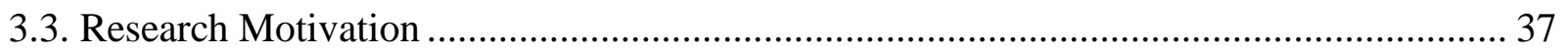

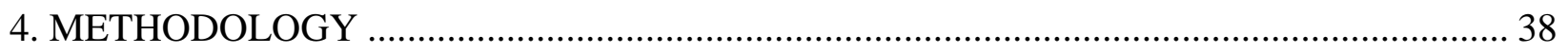

4.1. GIS Modeling of Solar Neighbourhood at a High Spatiotemporal Resolution ................ 39

4.1.2. Building of the 3D Model ................................................................................ 42 


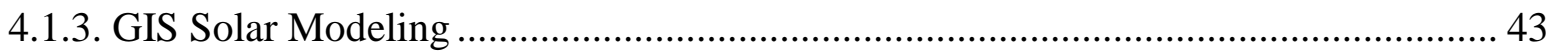

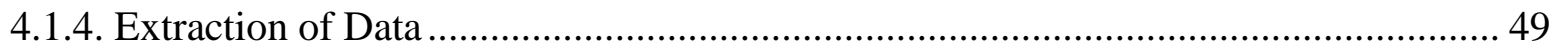

4.2. Benchmarking of 3D Solar Simulation Software Tools .................................................. 49

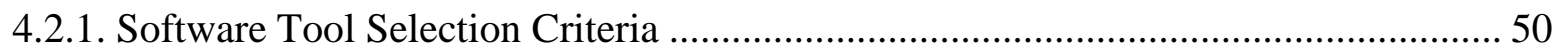

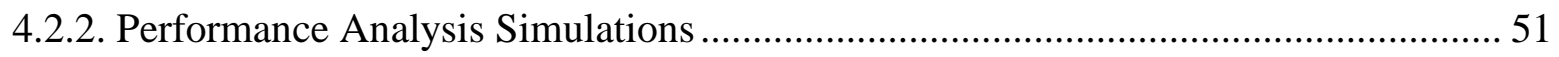

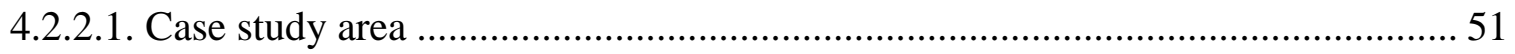

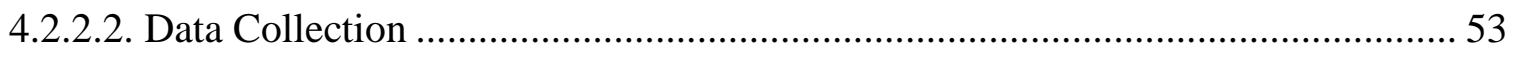

4.2.2.3. Simulation Procedures in the Software Tools................................................. 54

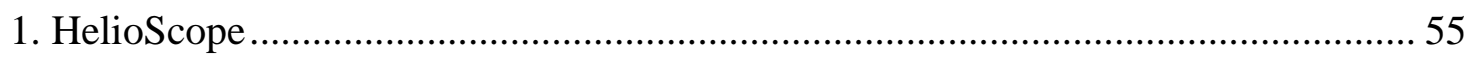

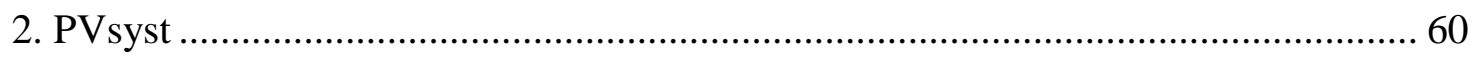

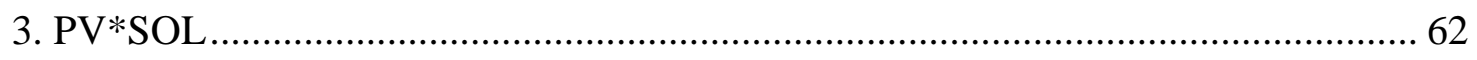

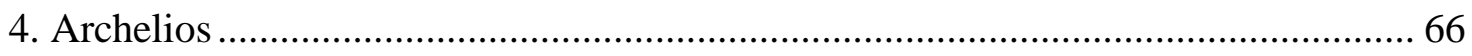

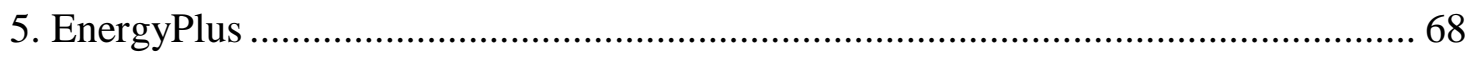

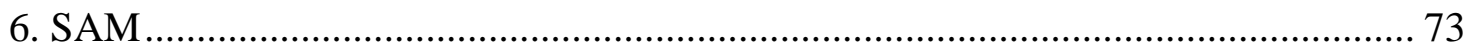

4.2.3. Physical Functions and Capabilities of the Software Tools......................................... 78

4.2.3.1. System Requirements and Availability ........................................................... 78

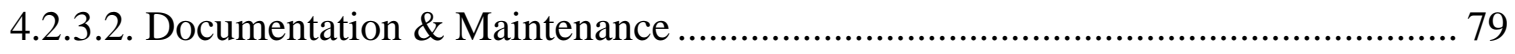

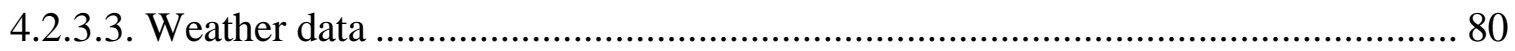

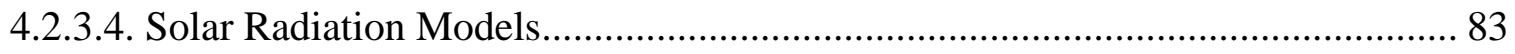

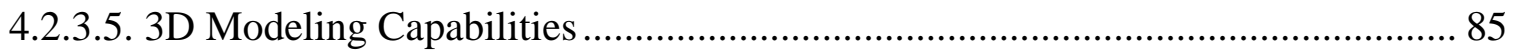

4.2.3.6. Shade Modeling ........................................................................................... 87

4.2.3.7. Maximum Modeling Capabilities .................................................................... 90

4.2.3.8. PV and Inverter Component Database ........................................................... 92

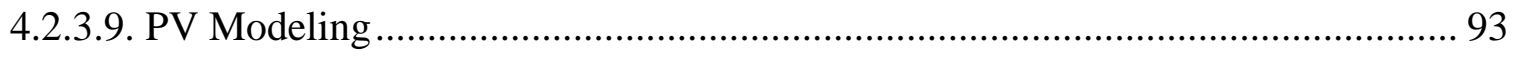

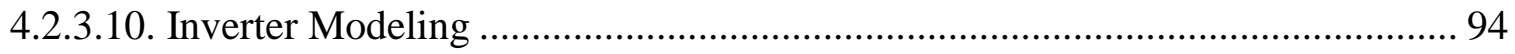

4.2.3.11. Modeling of System Losses ......................................................................... 95

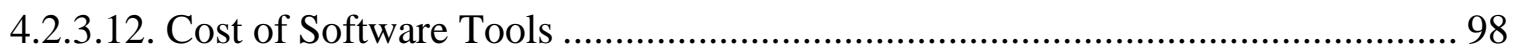

4.2.3.13. Report \& Analysis Output .......................................................................... 99

4.2.3.14. User-friendliness and level of expertise...................................................... 101

4.2.3.15. Modeling Flexibility .................................................................................. 102

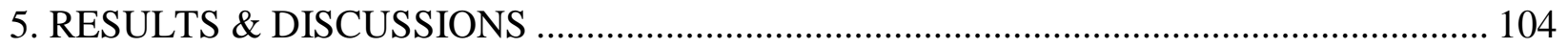

5.1. GIS Modeling of Solar Community Results .............................................................. 104

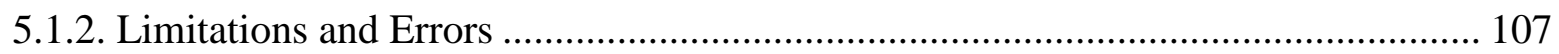

5.2. Benchmarking of Solar Simulation Software Tool Results .............................................. 108 


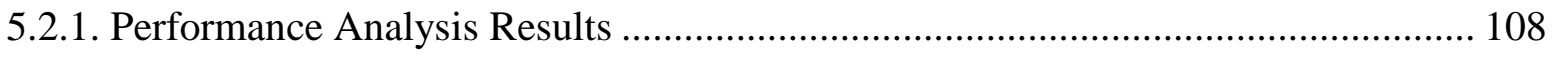

5.2.1.1. Annual PV Electricity Generation ............................................................... 110

5.2.1.2. Monthly PV Electricity Generation .................................................................. 111

5.2.1.3. Average Monthly Hourly PV Generation ...................................................... 115

5.2.2. Comparison of the Physical Functions and Capabilities of the Software Tools....... 122

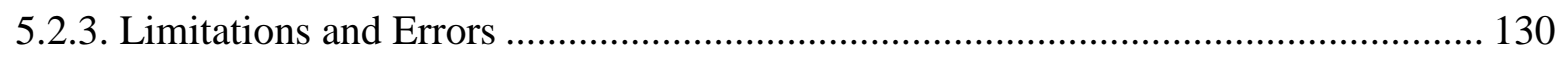

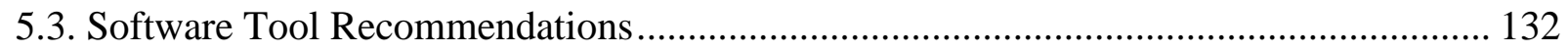

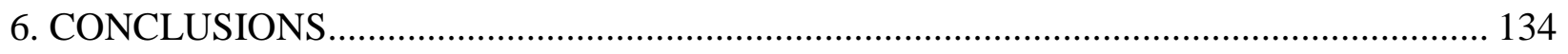

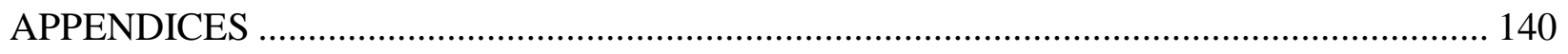

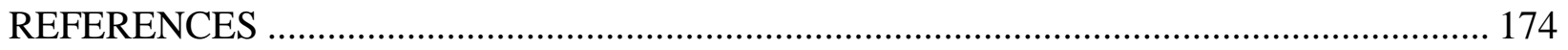




\section{List of Tables}

Table 1. Comparison of Benchmarking as an Empirical Method to Experiments and Case

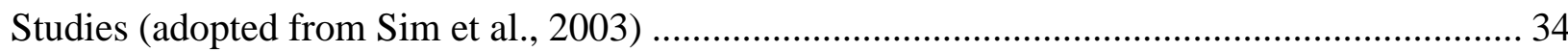

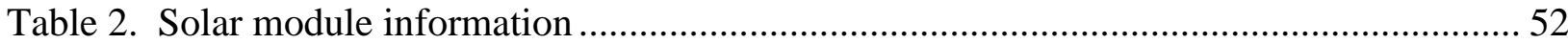

Table 3. PV Modeling Specifications ................................................................................ 52

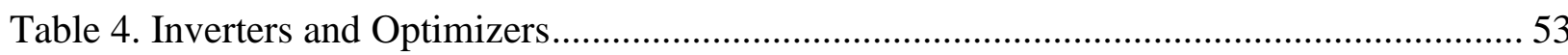

Table 5. Weather data information required for creating own solar resource data set in SAM. . 83

Table 6. Summary of DC loss values in SAM for central inverters, microinverters and DC

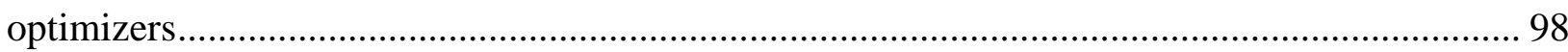

Table 7. Range of annual percentage error for HelioScope, SAM, PVSOL, EnergyPlus and PVsyst

Table 8. Monthly percentage error range and software tool the maximum and minimum was

predicted by.

Table 9. Comparison of the physical functions and capabilities for HelioScope, PVsyst,

PV*SOL, Archelios, EnergyPlus and SAM

Table 10. Software tools recommended for best physical functions and capabilities 


\section{List of Figures}

Figure 1. one-diode model (4 parameters) - considers shunt resistance infinite (adopted from Roberts et al., (2017)

Figure 2. One-diode model (5 parameters) - considers shunt and series resistance (adopted from

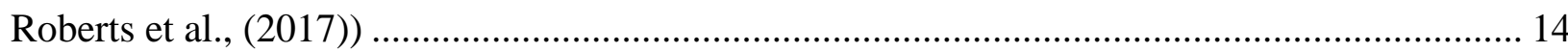

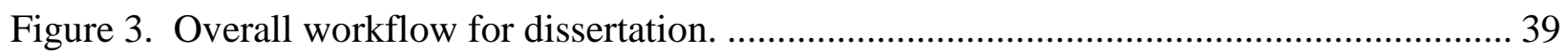

Figure 4. Methodology workflow for 3D GIS solar modeling of hourly solar potential ............. 41

Figure 5. 3D model imported into ArcScene in dae format...................................................... 42

Figure 6. Original site plan design - converted 2D raster digital elevation model (DEM) at a

resolution of $0.5 \mathrm{~m}$ converted from 3D file, projected onto orthophoto.

Figure 7. Converted 2D raster DEM at a resolution of $0.5 \mathrm{~m}$ converted from 3D file, projected onto orthophoto: (a) alternative community design, and (b) original case study design. 43

Figure 8. Graphic representation of the Area Solar Radiation Model for iterative analysis. ...... 47

Figure 9. Example of an annual solar insolation map generated from the methodology for the original site plan 48

Figure 10. Extruded 3D solar insolation map in ArcScene based upon Figure 5 and original DEM

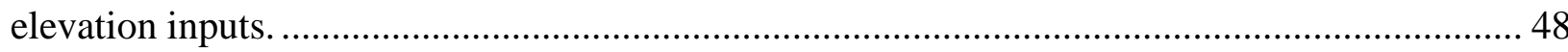

Figure 11. Photo of the case study residential home. ................................................................ 52

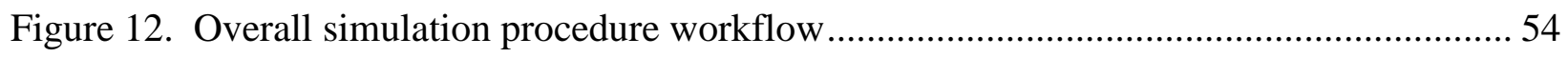

Figure 13. Mechanical layout design and selected parameters for case study area..................... 56

Figure 14. 3D view of case study area with neighbouring buildings and trees modeled as

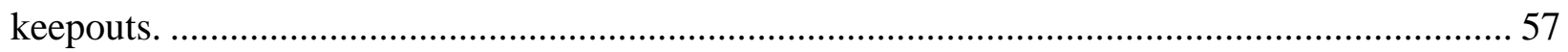

Figure 15. Electrical design layout of case study area with inverter and optimizers modeled.... 58

Figure 16. Condition Set 1 parameters - Perez transposition model.............................................. 59

Figure 17. Condition Set 2 parameters - Hay model.............................................................. 59

Figure 18. Imported 3D file of case study area into "near shadings" the 3D shading scene constructor for PV syst......................................................................................................... 61

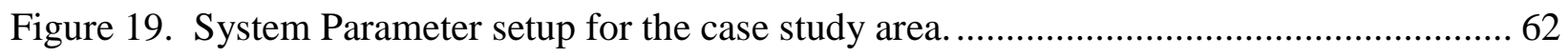

Figure 20. Case study house modeled in the 3D Editor of PV*SOL.......................................... 64

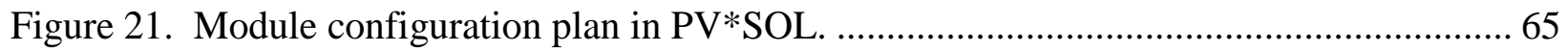

Figure 22. Case study area modeled in Trimble SketchUp with Archelios Pro plugin............... 66 
Figure 23. Irradiation computation in Archelios Pro for visualization of irradiation levels

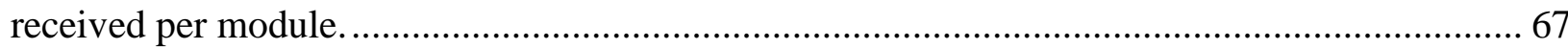

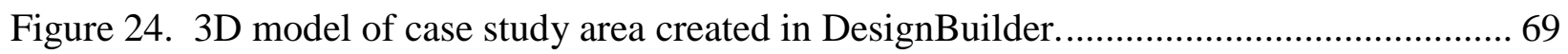

Figure 25. Simple PV model parameters used for simulation in DesignBuilder...................... 71

Figure 26. Equivalent one-diode model parameters for simulation of Canadian Solar CS6P-265P

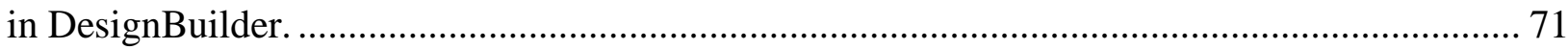

Figure 27. Electric load centre created for simulation......................................................... 72

Figure 28. 3D drawing representing the case study area in SAM's 3D shade calculator........... 76

Figure 29. Bird's eye view of case study area with Google maps underlay............................. 77

Figure 30. SAM 3D Shading Scene calculator depicting drawing capabilities....................... 87

Figure 31 . Monthly average hourly solar radiation of case study location in $\mathrm{Wh} / \mathrm{m}^{2}$............. 105

Figure 32. Percentage increase in average monthly solar radiation received for the alternative

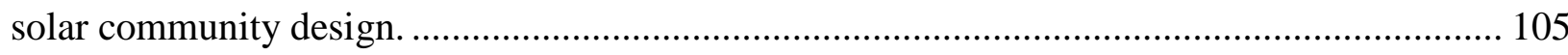

Figure 33. Average hourly solar radiation of case study location on the winter solstice. ......... 106

Figure 34. Average hourly solar radiation of case study location on the summer solstice......... 106

Figure 35. Percentage increase in average hourly solar radiation received for the winter and

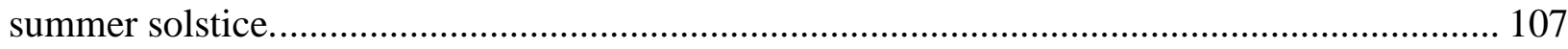

Figure 36. Simulations performed in each software tool................................................ 109

Figure 37. Annual modeled to measured percentage difference for HelioScope, SAM, PVSOL,

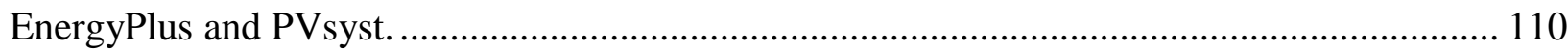

Figure 38. HelioScope monthly simulations compared to measured generation...................... 112

Figure 39. SAM monthly simulations compared to measured generation.............................. 112

Figure 40. PVSOL monthly simulations compared to measured generation........................... 113

Figure 41. EnergyPlus monthly simulations compared to measured generation..................... 113

Figure 42. PVsyst monthly simulations compared to measured generation........................... 113

Figure 43. Comparison of monthly percentage difference (modeled/measured -1$)$............... 114

Figure 44. Average monthly hourly PV generation for January......................................... 116

Figure 45. Average monthly hourly PV generation for February ...................................... 117

Figure 46. Average monthly hourly PV generation for March.......................................... 117

Figure 47. Average monthly hourly PV generation for April.............................................. 118

Figure 48. Average monthly hourly PV generation for May ............................................. 118 
Figure 49. Average monthly hourly PV generation for June........................................... 119

Figure 50. Average monthly hourly PV generation for July. ............................................ 119

Figure 51. Average monthly hourly PV generation for August. ......................................... 120

Figure 52. Average monthly hourly PV generation for September.................................... 120

Figure 53. Average monthly hourly PV generation for October. …................................... 121

Figure 54. Average monthly hourly PV generation for November. ................................... 121

Figure 55. Average monthly hourly PV generation for December...................................... 122 


\section{List of Appendices}

Appendix A. Example of GIS input parameters for Area Solar Radiation Model used for the

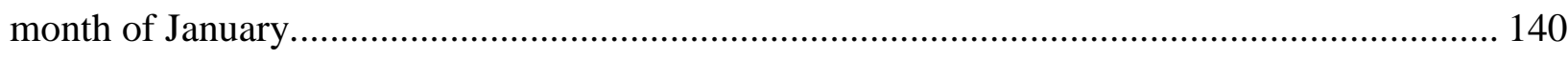

Appendix B. HelioScope Simulation Report ......................................................................... 142

Appendix C. PVsyst Simulation Report ............................................................................ 145

Appendix D. PV*SOL Simulation Report........................................................................... 151

Appendix E. Archelios Simulation Report ............................................................................... 163

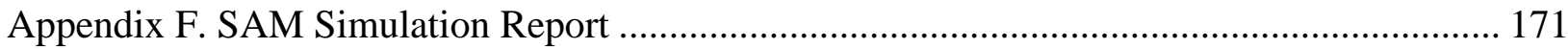




\section{List of Abbreviations}

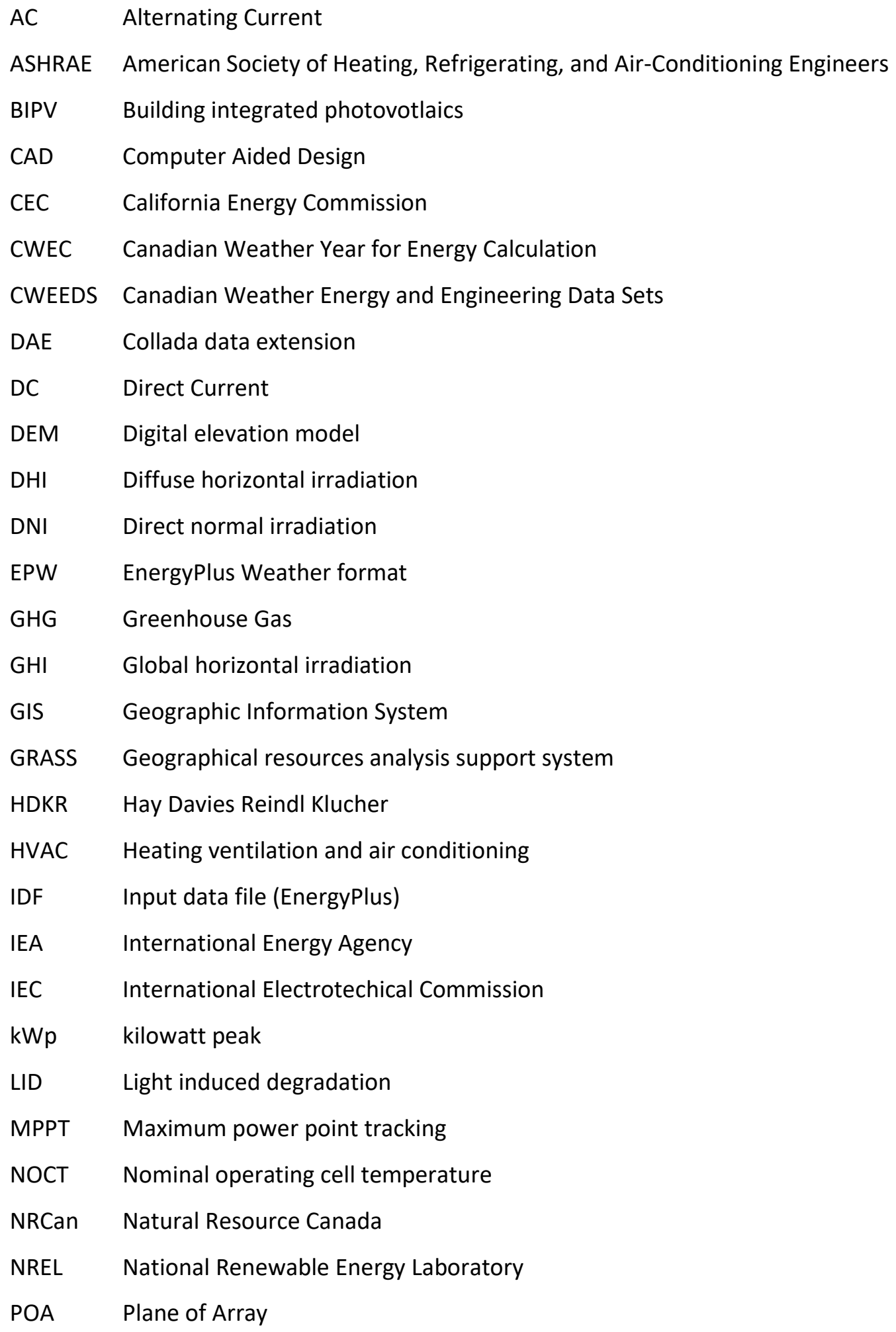




$\begin{array}{ll}\text { PV } & \text { Photovoltaic } \\ \text { SAM } & \text { System Advisor Model } \\ \text { STC } & \text { Standard Test Conditions } \\ \text { TMY } & \text { Typical Meteorological Year } \\ \text { TOU } & \text { Time-of-use }\end{array}$




\section{INTRODUCTION}

Cities, which approximately cover $2 \%$ of the Earth's surface, are responsible for over $80 \%$ of the world oil, gas and coal consumption and are the main contributors of greenhouse gas (GHG) emissions (Nouvel et al., 2014). Concerns over climate change and environmental sustainability present a challenge to shift and transform our traditional energy supplies that are based upon the usage of fossil fuels towards more renewable and non-polluting sources. Solar photovoltaic (PV) energy offers a sustainable way of providing society with a renewable source of energy and can help decrease the reliance on fossil fuel consumption. It is attracting increased attention from analysts in recent years due to the potential benefits it can yield towards a growing urban population, such as efficiency, economics, and environmental benefits (Budischak et al., 2013).

Buildings that are able to self-sustain themselves and use PV energy that is collected onsite are becoming an emerging trend. Net-zero energy buildings are buildings that generate as much energy from renewable sources as they consume in an average year (O'Brien et al., 2010). Renewable PV energy development and integration into communities can have a significant impact on the way electric utilities conduct their businesses moving forward. The adoption of solar communities has been relatively slow in Canada, due to associated high initial installation costs, and lack of policies to support the development of low carbon neighbourhoods. Advanced tools and simulations are needed in order to promote the benefits of net-zero solar communities and spur policy development in Canada.

The price of electricity in Ontario is controlled through time-of-use (TOU) pricing. This regime has divided electricity prices into three different TOU price periods: on-peak, mid-peak, and off-peak. These divisions are based upon the time of the day when electricity demand is at its highest, moderate and lowest, respectively. It is further divided into summer and winter pricing regimes. The peak generation of electricity from solar PV coincides with on-peak summer demand times in Ontario. This means that electricity produced by PV could help offset peak demands on the electricity grid and contribute to a more stable and sustainable energy supply. Hence, it is important to understand what effects the generation of PV so that electricity production could be maximized during these on-peak hours. There are many factors that effect the amount of solar PV electricity produced such as geographic location, system type and size, 
shading from nearby obstructions or buildings, available rooftop or surface area for installation, and the local environment. All these factors need to be understood and considered when estimating the production of PV electricity.

The goal of this dissertation is to increase the assessment ability of solar energy utilization and planning support for clusters of different types of buildings in a mixed-use community. Particular focus will be placed on the analysis of community-based modeling, mapping and forecasting of solar potentials on the rooftops of buildings. New systems and methodologies with appropriate level of detail at a lower computational time are needed to accurately model, estimate and map solar energy potential at a high spatiotemporal resolution. To accomplish this goal and to develop an integrated solution, the assessment ability was investigated using two different types of studies: (1) 3D GIS modeling of a solar energy community, and (2) benchmarking of solar PV radiation software tools.

A 3D GIS modeling approach was developed within ESRI ArcGIS to evaluate solar energy potential on a proposed mixed-use community, and six solar PV software tools (HelioScope, PVsyst, PV*SOL, Archelios, EnergyPlus, and System Advisor Model (SAM)) were evaluated. These six software tools were evaluated based upon their accuracy in simulation performance analysis, and the comparison of their physical functions and capabilities. Both case studies analyzed in this dissertation were located in southern Ontario, near the City of London. The end goal provided insights on how modeling and software tools could be used to support the planning and development of solar energy communities, and for the future development of a new hybrid software tool using GIS coupled with the internal submodels of PV solar radiation software tools.

This dissertation is organized as follows: (1) a detailed literature review regarding solar PV and its modeling process, (2) a discussion about the research objectives, (3) the developed methodology and approach, (4) a discussion about the results that were obtained from both case studies, and (5) the conclusions and areas for future improvement in this research. 


\section{LITERATURE REVIEW}

This chapter will begin by providing a discussion about the principles behind solar PV energy and solar PV production modeling. The meteorological data, solar radiation models, PV models, inverter models, and sources of system losses will be reviewed. The discussion will then focus on solar energy communities and the current state of research for urban rooftop and 3D rooftop and façade modeling. Lastly, this chapter will conclude with a discussion regarding the evaluation and current state of solar simulation software tools and software benchmarking.

\subsection{Solar Photovoltaic Energy}

Solar energy is one of the oldest energy sources ever used and has been recognized for a long time as a major source of renewable and sustainable energy. Becquerel was the first to discover the PV effect in selenium in 1839 (Kalogirou, 2014). The first practical PV applications were used in space in the 1950's, and it was not until the 1970's in which the interest in PV land-based applications exploded (Green, 2004).

Solar irradiance is defined as the intensity of solar radiation received on a surface at a given time and is usually expressed in Watts per square metre (W/m²) (CANMET, 1991). Whereas the term irradiation is defined as the incident energy per unit area on a surface, determined by the integration of irradiance over a specified time, such as an hour or a day (Duffie \& Beckman, 2013). The term insolation refers to the amount of solar energy received over a period of time (CANMET, 1991), which is specifically referring to solar energy irradiation (Duffie \& Beckman, 2013). Note that these terms will be used interchangeably in this thesis.

PV systems are comprised of cells that convert sunlight into electricity and are made up of various semiconductor materials that allow electrons to be freed from their atoms through sunlight exposure (NRC, 2002). This enables them to carry an electric current and ultimately produce electricity. Only some of the light that is received on the PV cell is converted into electrical energy. The rest is lost by reflection from the cell surface or in the form of heat (CANMET, 1991). PV systems are rated in peak power output (kWp), measured under standard test conditions (STC), which is the amount of electrical power that a system is expected to 
produce at solar noon on a clear day (Parida et al., 2011). The efficiency of a solar PV system is defined as the maximum electrical power output divided by the incident light power. Efficiency values for PV systems are generally recorded for a PV cell temperature of $25^{\circ} \mathrm{C}$ and incident light irradiance of $1000 \mathrm{~W} / \mathrm{m}^{2}$ and vary depending on the type of material used (Kalogirou, 2014).

Silicon is the major material used in making PV cells, due to its vast natural abundance on the earth and high efficiency (Tyagi et al., 2013). Crystalline silicone PVs dominates $80 \%$ of the market, and thin-film materials 20\% (Kalogirou, 2014; Tyagi et al., 2013). The materials most commonly used for PV production include silicon $(\mathrm{Si})$, compounds of cadmium sulfide (CdS), cuprous sulfide $\left(\mathrm{Cu}_{2} \mathrm{~S}\right)$, cadmium telluride (CdTe), copper indium diselenide (CIS) and gallium arsenide (GaAs) (Kalogirou, 2014; Tyagi et al., 2013; Parida et al., 2011).

A PV power generation system is comprised of multiple components including cells, mechanical and electrical connections, mountings, inverters and batteries (optional) (Parida et al., 2011). The overall performance of PV technologies does not just depend on these operating conditions, it depends on many other factors as well such as total available irradiation, geographic location (latitude), prevailing weather patterns, cloud haze, seasonal effects, orientation, inclination (tilt), and the local environment (Mardaljevic \& Rylatt, 2003; CANMET, 1991). The tilt of a PV array can significantly affect the amount of solar radiation received. PV panels are generally installed at a tilt equal to latitude, depending on the application. For example, a smaller tilt angle is sometimes used in order to minimize shading of neighboring modules, minimize wind load, and to increase radiation exposure in the summer months (Kalogirou, 2014).

The main environmental benefits that are associated with solar PV include: (i) reduced greenhouse gas emissions ( such as $\mathrm{CO}_{2}$ and $\mathrm{N}_{2} \mathrm{O}$ ) and prevention of toxic gas emissions $\left(\mathrm{SO}_{2}\right.$ and particulates), (ii) reclamation of degraded land, (iii) reduction of the required transmission lines of the electricity grids, (iv) improvement of the quality of water resources, and (v) generation of little noise during operation (Kaygusuz, 2009; Tsoutsos et al., 2005). If current growth trends continue for solar PV, it is projected that by 2050, PV could provide up to $11 \%$ of electricity worldwide, which would contribute to a reduction of $2.3 \mathrm{Gt}$ of $\mathrm{CO}_{2}$ emissions per year (IEA, 2010). 


\subsection{Solar Photovoltaic Production Modeling}

This section will be discussing the meteorological data, models and theories that are used in order to estimate solar PV energy production. This discussion will be broken down into: (1) meteorological data, (2) solar radiation models, (3) PV performance models, (4) inverter models, and (5) modeling of system losses.

\subsubsection{Meteorological Data}

Meteorological data sets are required in order to estimate the amount of solar radiation that lands on the PV array system. Weather data is specific per location as it varies by latitude, seasonality and prevailing weather conditions. Hence, having good weather data is very important for the accuracy of modeling of solar PV generation potential. Weather data can be measured from local ground-based weather stations, satellites, and even modeled for remote geographic locations. Meteorological data generally includes global horizontal solar radiation, direct beam solar radiation, horizontal diffuse solar radiation, temperature, cloud cover, wind speed and direction, along with other meteorological elements (Yates \& Hibberd, 2010). This type of data is generally expensive to collect, and relatively few weather stations collect this type of data (McKenney et al., 2008).

The most accurate weather data are derived from weather stations with well-maintained good quality instruments which represent the ground truth (Meteonorm, 2019). However, since weather stations are not installed everywhere and data can be missing, satellite data is then used. Satellite data are a valuable source of solar irradiation data as it can reach remote locations. Satellite data offers a good compromise to sparse ground data, and can be obtained from government agencies, such as NASA, or private companies which offer finer resolution (in time and space) such as SolarGIS and Clean Power Research (IEA, 2017). However, there are uncertainties to this approach due to lack of meteorological parameters, uncertainties in aerosol values, detection of multiple cloud layers, inaccuracies in areas with snow or no data north of $62^{\circ}$ (Meteonorm, 2019). In certain circumstances, when data is not available for a specific location, it is also possible to use insolation data from the station nearest the proposed site, or to interpolate between two or three stations within the vicinity (CANMET, 1991).

Weather data for a representative year used in building energy simulation programs are generally presented as typical meteorological year (TMY) or test reference year (TRY) files. 
TMY data sets have an annual data set that contains hourly meteorological values for typical conditions over a long period of time (approximately 30 years) (Wilcox \& Marrion, 2008). It should be noted that TMY data represent long term averages and are not indicative of a specific year's performance. In regard to solar radiation modeling, TMY data contain three solar radiation values and they are the total horizontal, direct beam, and horizontal diffuse radiation (Cameron et al., 2008). Most simulation software tools commonly use TMY, TMY2 or TMY3 datasets for input values. TMY2 are updated from TMY, and TMY3 data were later used to update TMY2 data. TMY2 weather files are widely used in simulations such as EnergyPlus and TRNSYS (Kalogirou, 2014). TMY2 and TMY3 files contain hourly records for solar radiation, dry bulb temperature, illuminance, precipitation, visibility, and snowfall. These weather data sets are available for more than 2100 locations globally (U.S. DOE, 2013).

For Canadian locations, TMY data used for building energy simulation are from the Canadian Weather for Energy Calculations (CWEC). This data is derived from the Canadian Weather Energy and Engineering Datasets (CWEEDS), which contain hourly weather data such as solar irradiance, temperature, dew point, pressure, wind speed and other relevant elements for building and solar energy simulations and design. The files were created for providing long-term weather records for use specifically in urban planning, design of energy efficient buildings, solar renewable energy systems and any areas of weather applicable studies (Environment Canada, 2008). The most up to date version of the CWEEDS/CWEC data was released in 2016, which provided new and updated files for 492 Canadian locations representing at least ten years of data from the period of 1998 - 2014 (Morris, 2016). Prior to this release, the last major release of CWEEDS files from Environment Canada was in 2005. For locations where data is not available, a tool called Meteonorm can be used to interpolate and extrapolate weather data for simulation.

Meteonorm is a meteorological database that contains a comprehensive database of weather data for almost any geographic location on the earth. It is a global climate database and generates accurate and representative typical years for any place on earth. The database is comprised of over 8000 weather stations and five geostationary satellites. It also uses interpolation models based on data from over thirty years of data for high accuracy datasets, which can calculate minute and hourly data for radiation parameters on inclined planes. Meteonorm measures over thirty different meteorological parameters such as global radiation, 
temperature, humidity, precipitation, days with precipitation, wind speed and direction, and sunshine duration, to name a few. Meteonorm is a versatile tool as it can generate thirty-six predefined output formats that cover industry standard simulation software in building design and energy, as well as user-defined output format if desired. Examples of these outputs include popular simulation formats such as TMY2, TMY3, EPW, TRNSYS, TRY, PVSOL, and PVsyst (Meteonorm, 2019). Meteonorm is a proprietary commercial software tool and is available at a fee.

\subsubsection{Solar Radiation Models}

Typical meteorological data generally include three values for solar radiation measured on a horizontal surface: direct normal (also commonly called beam) irradiance (DNI), global horizontal irradiance (GHI), and diffuse horizontal irradiance (DHI). The accuracy of a solar radiation model is affected by the weather at the geographical location and the quality of the weather data (Yates \& Hibberd, 2010). Difficulties are sometimes encountered with the measuring of the three components since most ground-based meteorological stations measure primarily global horizontal radiation (sum of beam and diffuse horizontal irradiance) (Liu \& Jordan, 1960). Hence, it is common practice to estimate the direct and diffuse components from GHI through the use of decomposition models. Decomposition models are used to estimate the DNI and DHI from measured global horizontal irradiance (GHI). Common ones include: Orgill and Hollands, Erbs et al. model, Maxwell model (DISC) and Ineichen et al. (DIRINT), and Reindl et al. (Roberts et al., 2017). Muneer (2004) provides a thorough discussion regarding these various models. Ideally, it is best to have all three components measured (if possible) in order to avoid additional uncertainty from the usage of the decomposition models.

In order to determine the amount of solar irradiance that a tilted surface receives, models are required to convert the amount received horizontally to that of the orientation and tilt of the PV panel commonly referred to as the plane of array (POA). Knowledge of this data is particularly important for PV type applications, since PV systems are generally installed on tilted surfaces in order to increase the amount of solar radiation received and to reduce reflection and cosine losses (Kalogirou, 2014). Calculation of direct (beam) energy on a slope when its value on the horizontal is known is a straightforward calculation based upon solar geometry, however, estimation of the diffuse component at a specific orientation and tilt, is not as simple. In order to 
estimate the amount of irradiance received on the POA the usage of transposition models are required. Transposition models refer to the calculation of the incident irradiance on a tilted plane, based upon the horizontal irradiance data.

There are three main components of solar radiation that are used in solar modeling algorithms and these are the beam (or direct), diffuse, and ground-reflected components (Perez \& Stewart, 1986). The basic input parameters that are required in order to generate the global irradiance received by a tilted plane for all irradiance models are: global horizontal irradiance $\left(\mathrm{G}_{\mathrm{h}}\right)$, direct normal irradiance $(\mathrm{I})$, diffuse horizontal irradiance $\left(\mathrm{D}_{\mathrm{h}}\right)$, the location's latitude $(\phi)$, the slope of the plane $(\beta)$, azimuth $(\gamma)$, Julian date $(\mathrm{n})$, true solar time $\left(\mathrm{t}_{\mathrm{s}}\right)$, and the surrounding ground albedo $(\rho)$ (Perez \& Stewart, 1986). The differences in models are mainly contributed to the way in which the three components of energy are handled which are the direct beam, skydiffuse and the ground-reflected components. Thus, the hourly energy $\left(\mathrm{G}_{c}\right)$ on a tilted plane is given by

$$
G_{c}=I_{c}+D_{c}+R_{c}
$$

where $I_{c}, D_{c}$ and $R_{c}$ are the direct, sky-diffuse and reflected components respectively (Perez \& Stewart, 1986).

Beam radiation is defined as the solar radiation received from the sun without having been scattered by the atmosphere (Duffie \& Beckman, 2013). Commonly referred to as the direct beam component, it is generally the easiest to calculate and its algorithm is identical in all models (Perez \& Stewart, 1986). The source of error for direct radiation is generally negligible provided that accurate input data are used. The direct beam component $I_{\mathrm{c}}$, can be found from direct irradiance $I$ and is defined as:

$$
I_{c}=I\{\max (0, \cos \theta)\}
$$

where $\theta$ is the solar incidence angle, $\cos \theta$ is defined as:

$$
\begin{gathered}
\cos \theta=\sin \delta \sin \phi \cos \beta-\sin \delta \cos \theta \sin \beta \cos \gamma+\cos \delta \cos \phi \cos \beta \cos \omega \\
+\cos \delta \sin \phi \sin \beta \cos \gamma \cos \omega+\cos \delta \sin \beta \sin \gamma \sin \omega
\end{gathered}
$$


and $\omega$ is the hour angle obtained from $\mathrm{t}_{\mathrm{s}}, \delta$ is the solar declination defined as:

$$
\delta=23.5^{\circ} \sin \left[\frac{(284+n) 360}{365}\right]
$$

and $\omega=180^{\circ}$ starting at midnight and decreases by $15^{\circ}$ per hour (Perez \& Stewart, 1986).

Diffuse or (sky-diffuse) radiation is defined as the solar radiation received from the sun after its direction has changed due to scattering in the atmosphere (Duffie \& Beckman, 2013). It refers to that part of the solar radiation arriving at the Earth's surface after first being scattered by obstructions in the atmosphere, such as by haze, dust, and reflection by natural and man-made surfaces (water, buildings, and mountains) (Rylatt et al., 2001). The diffuse component of solar radiation is very difficult to accurately model, as the distribution of radiance throughout the sky is very hard to replicate. The estimation of diffuse solar radiation is considered to be the predominant source of error associated with the models and where the main discrepancies arise (Perez \& Stewart, 1986). Sky models are needed in order to calculate the diffuse solar radiation and the most commonly used sky models are the circumsolar (clean and cloudless skies), isotropic (overcast skies), and anisotropic models (partly cloudy skies) (Chow et al., 2005; Pandey \& Katiyar, 2009).

\section{Circumsolar Model}

The Circumsolar Model theory is based upon clean and cloudless skies. The main assumption for this model is that all radiation arriving on a horizontal surface comes from the direction of the sun. In order to calculate the diffuse component for a tilted plane the following relationships are used:

$$
D_{c}=D_{h} r_{b}
$$

where $r_{b}$ is the ratio of the daily direct solar radiation incident on a tilted surface to that on a horizontal surface denoted as:

$$
r_{b}=\frac{\sin \delta \sin (\phi-\beta)+\cos \delta \cos (\phi-\beta) \cos \omega}{\sin \delta \sin \phi+\cos \delta \cos \phi \cos \omega}
$$


(Pandey \& Katiyar, 2009).

\section{Isotropic Model}

The Isotropic Model is the simplest model of them all and it assumes that the intensity of the diffuse component of irradiance is equal in all parts of the sky. Isotropic models are based on the assumption that the intensity of the diffuse sky irradiance is uniform over the entire sky dome, and the diffuse irradiance is the same regardless of direction/orientation. It only depends on the fraction of the sky dome that is seen by the surface. The isotropic sky distribution of light throughout the sky dome is the most popularly used method for calculating diffuse solar radiation (Perez \& Stewart, 1986). This model is more realistic than the circumsolar model as it does not only assume only for a clean sky. The isotropic model approximates conditions for overcast (cloudy) skies and is based upon the assumption that the intensity of the sky diffuse radiation is uniform over the entire sky dome (Pandey \& Katiyar, 2009; Ineichen 2011). This model simply defines diffuse irradiance as

$$
D_{c}=\frac{1}{2} D_{h}(1+\cos \beta)
$$

It is because of the acceptable level of errors that this model produces and its simplicity that it is widely used (Perez \& Stewart, 1986). The isotropic model tends to produce more conservative estimates (Duffie \& Beckman, 2013).

\section{Anisotropic Model}

The Anisotropic Model was developed after the isotropic model in order to refine its simple algorithm to accommodate the need for more accurate modeling as time progressed (Perez \& Stewart, 1986). The anisotropic model incorporates conditions for partly cloudy skies by multiplying the original isotropic algorithm by a new function. Anisotropic sky models assume that the diffuse sky irradiance is not uniform and is anisotropic at the horizon and in the circumsolar region. There exist many sky diffuse models, and some of the most commonly used anisotropic sky models include the Hay model (Hay, 1979), Perez model (Perez et al., 1987), Klucher model (Klucher, 1979), and Reindl model (Reindl, 1990) (Ineichen, 2011). A detailed review of most of these sky models can be found in Hay \& McKay (1985). A brief highlight of 
some of the more common models will be described below for the Klucher, Hay, and Perez models.

\section{Klucher model}

The Klucher (1979) anisotropic model was inspired by the isotropic model and the Temps \& Coulson (1977) model. The Temps \& Coulson (1977) model was an algorithm based upon clear day models for all sky conditions and applied a correction factor of $\left[1+\sin ^{3}\left(\frac{\beta}{2}\right)\right]$. Klucher refined their algorithm by incorporating conditions for cloudy skies by adding the factor $\mathrm{K}$ to get the following equation

$$
D_{c}=D_{h}(1+\cos \beta) \frac{1}{2}\left(1+K \sin ^{3}\left(\frac{\beta}{2}\right)\right)\left(1+K \cos ^{2} \theta \sin ^{3} Z\right) \text {. }
$$

Where $\mathrm{K}$ is a modulating function representing the degree of anisotropy and is used to account for cloudiness denoted by

$$
K=1-\left(\frac{D_{h}}{G_{h}}\right)^{2}
$$

When $\mathrm{K}=0$, it simulates conditions for overcast skies, and when $\mathrm{K}$ approaches 1 it simulates conditions for clear skies. Thus, the Klucher model is able to account for partly cloudy skies by taking into consideration the anisotropic effect of both circumsolar and horizon brightening with the isotropic effect. Note that when $\mathrm{K}=0, \mathrm{D}_{\mathrm{c}}$ becomes isotropic, and when $\mathrm{K}$ tends to $1, \mathrm{D}_{\mathrm{c}}$ accounts for clear sky conditions (Pandey \& Katiyar, 2009).

\section{Hay model}

Hay's model assumes diffuse radiation incident on a horizontal surface to be composed of circumsolar and uniform background sky-diffuse components (Muneer, 2004). Hay’s anisotropic model is based upon an isotropic and circumsolar component and is defined by

$$
D_{c}=D_{h}\left\{\frac{H-D_{h}}{H_{o}} r_{b}+\frac{1}{2}(1+\cos \beta)\left[1-\frac{H-D_{h}}{H_{o}}\right]\right\}
$$


Where $\mathrm{H}$ is daily total solar on a horizontal surface, and $\mathrm{H}_{\mathrm{o}}$ is the daily extraterrestrial solar radiation on a horizontal surface (Pandey \& Katiyar, 2009). The Hay model takes into account the effects of terrestrial direct radiation to extraterrestrial radiation, and this is where the differences occur in the algorithm as compared to the prior equations mentioned.

\section{Perez model}

The Perez (1986) anisotropic model is based on the analysis of three components in order to create a more detailed sky. The three dimensions take into account the simple representation of the sky dome, the effects of the circumsolar and horizon anisotropy factors (F1 and F2), and the $3 \mathrm{D}$ representation of those factors. The model can be basically described as a "sky hemisphere superimposing a circumsolar disc and horizon band on an isotropic background" (Chow et al., 2005). The Perez algorithm is then given as

$$
D_{c}=D_{h}\left[\left(1-F_{1}\right)\left(\frac{1+\cos \beta}{2}\right)+F_{1} \frac{a}{b}+F_{2} \sin \beta\right.
$$

Where $F_{1}$ and $F_{2}$ are empirical functions of the sky clearness and describe the circumsolar and horizon brightness respectively. The coefficients $a$ and $b$ account for the angle of incidence of the sun onto the inclined surface, where $a=\max \left(0, \cos (\theta)\right.$ and $b=\max \left(\cos 85^{\circ}, \cos \left(\theta_{z}\right)(\right.$ Roberts et al., 2017).

\section{$\underline{\text { Reflected component }}$}

Lastly, the ground-reflected component is considered to be challenging to model with great accuracy, but generally has the least amount of weight in the determination of hourly energy (as compared to the other two components of direct and diffuse radiation). The simplest approach to modeling the reflected component can be given by

$$
R_{c}=G_{h} A(1-\cos s) / 2
$$

where $\mathrm{A}$ is albedo and usually given a value of 0.2 or 0.7 for bare ground and snow covered respectively (Perez \& Stewart, 1986). The two main problems with estimating the ground- 
reflected radiation are the uncertainty of average reflectance of the neighbouring ground and the lack of an accurate model. Accurate estimation would require the detailed knowledge of the foreground type and geometry, its reflectivity, degree of isotropy, and details about the surrounding obstructions and skyline (Muneer, 2004).

\subsubsection{Photovoltaic Performance Models}

Photovoltaic performance models are mathematical representations used to estimate the energy yield of PV systems. PV performance models are used to determine how effective a PV system is at converting incident solar radiation on an array into usable power (Yates \& Hibberd, 2010). There are various models that are used to predict the I-V performance of a PV cell, module or array, ranging in various levels of complexity. The simplest method is to use the efficiency of the PV module, however, PV cell behaviour is most commonly represented using an equivalent circuit model which is composed of a current source, one or two anti-parallel diodes, with or without an internal series resistance, and a shunt/parallel resistance (Ma et al., 2014). The equivalent one-diode model is the most commonly employed model in engineering applications, as it offers a good compromise between simplicity and accuracy (Roberts et al., 2017). The two most commonly used variations of the one-diode model are the 4-parameter and 5 parameter models. The parameters that are used in the one-diode model are the: light current $\left(\mathrm{I}_{\mathrm{L}}\right)$, diode saturation current $\left(\mathrm{I}_{\mathrm{o}}\right)$, series resistance $\left(\mathrm{R}_{\mathrm{S}}\right)$, the shunt resistance $\left(\mathrm{R}_{\mathrm{sh}}\right)$, and the diode ideality factor $(\mathrm{n})$.

The one-diode 4-parameter model takes into consideration the series resistance case, in which the parallel resistance is considered as infinite and thus its effect is not taken into account. The 4-p model ignores the effects of the shunt resistance and has been found to be not as accurate as the 5-p model, as it does not fully reflect the effect of high temperatures on the current, and hence leads to a less accurate estimate of current (Ma et al., 2014). Its equation is given by:

$$
I=I_{L}-I_{D}-I_{S h}=I_{L}-I_{o}\left[\exp \left(\frac{V+I R_{S}}{n V_{t}}\right)-1\right]
$$

Where $\mathrm{V}_{\mathrm{t}}=\mathrm{N}_{\mathrm{c}} \mathrm{T}_{\mathrm{c}} \mathrm{k} / \mathrm{q}$ is the module thermal voltage with $\mathrm{N}_{\mathrm{c}}$ cells connected in series,

$\mathrm{T}_{\mathrm{c}}[\mathrm{K}]$ is the cell temperature 
$\mathrm{k}$ is the Boltzmann's constant $\left(1.381 \times 10^{-23} \mathrm{~J} / \mathrm{K}\right)$

$\mathrm{q}$ is the electron charge $\left(1.605 \times 10^{-19} \mathrm{C}\right)$.

The one-diode model 5-parameter model improves upon the accuracy of the one-diode 4-p model and takes into consideration parallel resistance as well as the series resistance. This is commonly called the five-parameter (5-p) model. Overall, the addition of the parallel resistance effect reduces the available electrical current, and the series resistance affects the output voltage (Ma et al., 2014). Its equation is given by:

$$
I=I_{L}-I_{D}-I_{S h}=I_{L}-I_{o}\left[\exp \left(\frac{V+I R_{S}}{n V_{t}}\right)-1\right]-\frac{V+I R_{S}}{R_{S h}}
$$

Figure 1 and Figure 2 depict the schematics for the one-diode model 4 and 5 parameter models respectively.

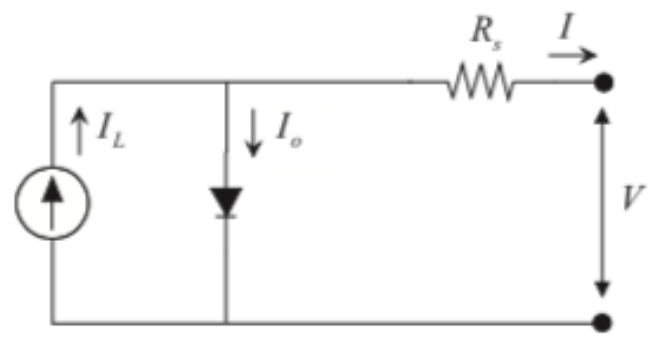

Figure 1. one-diode model (4 parameters) - considers shunt resistance infinite (adopted from Roberts et al., (2017)

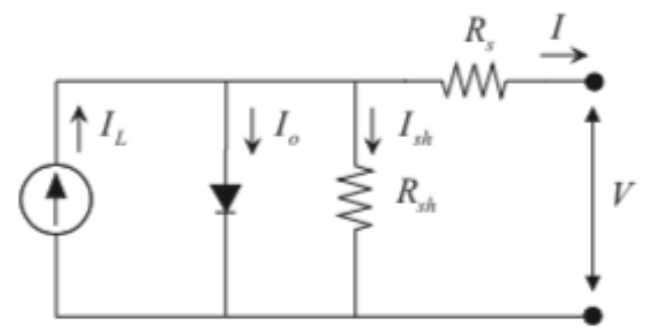

Figure 2. One-diode model (5 parameters) - considers shunt and series resistance (adopted from Roberts et al., (2017))

There are more complicated PV performance models that exist which incorporate more than one diode (IEA, 2017). For example, the two-diode model introduces an additional diode to the calculation which increases computational complexity significantly by increasing the number 
of coefficients required. The two-diode model is a non-linear equation and contains two exponential terms with up to seven unknown parameters (Ma et al., 2014). Hence, models with more than one-diode are not as frequently used for modeling.

Another example of a more complicated PV performance model is the Sandia model developed by Sandia National Laboratories in 2004 (Yates \& Hibberd, 2010). The model incorporates over thirty coefficients derived from outdoor testing conditions. The model includes four temperature coefficients, a polynomial representation of air mass, incident angle modifier, and a thermal model for cell and module temperature. This model is only used where testing for specific modules have already been done (Cameron et al., 2008).

\subsubsection{Inverter Models}

An inverter is used to convert the electricity output from direct current (DC) to alternating current $(\mathrm{AC})$. Inverters are rated by their total power capacity and rated by efficiency. The conversion of electricity from DC to AC represents a loss in the system and affects the performance of the system (Kalogirou, 2014). In most cases, the inverter efficiency is represented as a constant factor, which assumes that loss is linear over the operation range. However, this is not the case in real life situations, as it relies on the input voltage and on the load fraction at which the equipment is subjected (Roberts et al., 2017). Inverter efficiency varies with both DC power level and DC voltage, and this variation is heavily reliant on the manufacturer and inverter design (IEA, 2017). In general, the inverter is characterized by a power dependent efficiency $\mathrm{N}_{\mathrm{inv}}$, which is given by:

$$
N_{\text {inv }}=\frac{P_{\text {out }}}{P_{\text {in }}}=\frac{V_{a c} I_{a c} \cos (\varphi)}{V_{d c} I_{d c}}
$$

where

$\cos (\varphi)=$ power factor

$\mathrm{I}_{\mathrm{dc}}=$ current required by the inverter from the DC side

$\mathrm{V}_{\mathrm{dc}}=$ input voltage for the inverter from the DC side (Kalogirou, 2014).

Many methods exist to represent this electrical behaviour of inverters, from simple efficiency approximations based on manufacturers datasheet info, empirically derived equations, to more 
complex analytical based approaches to create a more accurate response of the inverter (Roberts et al., 2017).

The most commonly used approach in simulation software tools for modeling the behaviour of the inverter is to define the inverter's efficiency curve through the usage of manufacturer's data sheets and values. It uses the information for the maximum power rating, MPPT voltage range, the threshold power and the inverter's efficiency at various levels of loading (Yates \& Hibberd, 2010). More detailed models that are based upon measurements made at testing labs that measure efficiency at specific DC power and voltage levels exist as well, such as the Sandia inverter model (IEA, 2017). Again, usage of the Sandia inverter model is only available for inverter models in which test have been performed on.

\subsubsection{Modeling PV System Losses}

In real life, PV systems experience system losses in electricity output that need to be accounted for in the modeling process. These causes of system losses are commonly referred to as derate factors. Derate factors are a scaling factor that is applied to the PV array power output in order to account for the reduced electrical output in actual real-world operating conditions versus standard testing conditions (STC) (Roberts et al., 2017). Depending on the complexity of the model used for accounting for system losses, they can be either all grouped together or analyzed separately (for more accuracy). The following is a summary of the most common causes of system losses:

- Shading - Is very challenging to model and can significantly affect the output of a PV system. When a single derate factor is used to represent shading losses, this factor assumes that the shading losses are the same for every hour of the year. Most modeling tools tend to assume that the effects of shading are linear as well, which means that if $10 \%$ of the PV array is shaded, then you will lose exactly $10 \%$ of electricity production which is not a reflection of reality, as one single shaded cell on a PV can impact the whole module unproportionally (Yates \& Hibberd, 2010).

- Soiling losses - Effects vary depending on geographical location and weather conditions. In general, factors such as frequency and amount of precipitation, dew characteristics, wind patterns, snow, dust, proximity to sandy locations and so forth (Andrews \& Yates, 2015). Certain soiling losses are seasonal, such as snow losses. 
- Cell Temperature - module change temperature in response to changes in the POA irradiance, ambient air temperature, wind speed, and relative humidity. In general, most PV cells lose efficiency as temperature rises and typical rates are -0.3 to $-0.5 \%$ per ${ }^{\circ} \mathrm{C}$ above STC (IEA, 2017)

- DC losses - module nameplate DC rating, DC wiring, diodes and connections, mismatch, MPP tracker efficiency

- $\mathrm{AC}$ losses - AC wiring and transformer losses

- Mismatch losses - two main sources of mismatch losses are current mismatch and voltage mismatch. Current mismatches happen when the module that produces the least current limits the current in a PV source circuit, reducing its overall efficiency. (Andrews \& Yates, 2015)

- Other losses - light induced degradation (LID), cosine losses, angle of incidence, system aging, system availability

\subsection{Solar Energy Communities}

The concern over climate change and sustainability has motivated many community energy plans in Canada, in order to reduce GHG emissions and to become more energy self-sufficient (St. Denis \& Parker, 2009). This emerging trend requires communities to be able to generate a huge amount of its energy consumption through renewable technologies, such as PV. Renewable energy communities can be defined as:

A state-of-the-art community in which integrated, renewable energy technologies play the primary role in meeting the energy supply and demand needs of its residents, with the possibility of providing excess energy back to the grid or other communities. At a minimum, this community will have near zero or zero energy homes, integrated transportation modes with advanced vehicles, local renewable energy generation, and incorporate sustainable living practices (Carlisle et al., 2008).

Net-zero energy buildings are buildings that are able to generate as much energy from renewable energy sources as they consume in an average year (O’Brien et al., 2010). In general, most net- 
zero energy buildings will use a combination of solar PV technologies and energy efficient construction to be net-zero (Carlisle et al., 2008).

Micro-grids or community based renewable power generation systems provide many benefits as they can use centralized or decentralized (distributed) sources of power (Carlisle et al., 2008). If a community of a group of buildings is considered as a whole, optimal energy system infrastructure could exist due to the potential benefits of diversity in energy generation and demand. Grid connected PV systems in urban communities can help with electricity peak shaving, minimization of transmission and distribution losses and to increase grid capacity (Erdelyi et al., 2014). However, it is important that urban morphological layouts be considered for ideal orientation for PV installation.

There is a relationship between urban morphology and environmental sustainability (Sarralde et al., 2015). The impact of urban form on the solar potential of the community as a whole is important to consider, as mutual shading from buildings can cause significant losses in solar gain. Hence, parameters related to the urban form of neighbourhoods should be taken into consideration for realistic energy demand estimations (Tereci et al., 2013; Sarralde et al., 2015). Important design parameters that need to be considered for optimal solar neighbourhood design include the geometric shape of individual building units, density, and site layout (Hachem et al., 2012). The amount of solar access depends also on spatial and temporal factors. When analyzing solar potential of the community, it is important to keep the following concepts in mind: how to design building shapes, how to avoid mutual shading of buildings, and how to take into consideration the shape of the street (Hachem et al., 2011). It is for these reasons that detailed solar radiation modeling of the community's potential at a high spatiotemporal resolution is important, to see how various parameters affect the distributions in electricity generation potential.

\subsection{Urban Rooftop Solar Energy Modeling}

Solar radiation models are needed in order to estimate the amount of incoming solar irradiance on a particular location or surface. The urban environment is composed of complex 3D geometric objects and provides a series of challenges for the estimation of solar potential, as most existing models do not accurately consider the effects caused by urban shading, such as trees and building obstructions (Erdelyi et al., 2014; Esclapes et al., 2014). The major factors that 
affect the amount of solar irradiation received on a surface are geographic location, prevailing climatic conditions, urban micro-climate factors, orientation and the local environment (Mardaljevic \& Rylatt, 2003). Past research in this area used a variety of methods varying in levels of complexity and expertise.

There are various 2D commercial simulation software tools that provide quick estimates for solar potential without the need for the exact geometry of the rooftops or buildings. These decision-making software tools such as RETScreen and HOMER, are popularly used and provide quick estimations for solar PV potential based upon the location, climatic data, PV size, and PV input parameters for the calculation of PV electricity potential and financial returns. However, these tools are not able to consider the complex geometries of an urban neighbourhood or take into consideration shading caused by surrounding objects and trees.

Over the past two decades, geographic information systems (GIS) based approaches have been commonly used for large-scale solar radiation analysis of topographic (Dubayah \& Rich, 1995) and urban areas (Esclapes et al., 2014). GIS are powerful tools for analyzing and visualizing systems and have been extensively used in energy applications and infrastructures planning (Ramirez-Rosado et al., 2011). These GIS based solar radiation models can perform analyses on large scales using digital elevation models (DEM) or digital terrain models (DTM) and selected ground based or satellite data (Hofierka \& Zlocha, 2012).

Much literature has been published using GIS for urban rooftop solar modeling applications, ranging from the community level scale to large-scale analysis (e.g., Izquierdo et al., 2008; Jochem et al., 2009; Wiginton et al., 2010; Bergamasco \& Asinari, 2011; Nguyen \& Pearce, 2012; Strzalka et al., 2012; Chow et al., 2014; Kucuksari et al., 2014; Santos et al., 2014; Mainzer et al., 2017). These studies based upon GIS solar analysis functions use the topographic information that is stored in aerial images or DEMs to determine features such as individual rooftops, elevation, surface orientation and shadow casting. Based upon the desired extracted features these methods then estimate the solar radiation at every point of the DEM (Ruiz-Arias et al., 2009).

Many advances in the development of online mapping-based solar estimation tools have been made as well for quantifying solar PV potential and informing the public about the benefits and costs associated with PV. A few examples include PVGIS, Solar Boston, In My Backyard, PVWatts, San Francisco Solar Maps and Woodstock Solar Map (Chow et al., 2013). GIS based 
solar radiation models can be very beneficial to researchers because of their ability to map and interpolate complex spatial information. However, traditional GIS solar radiation models can only partially address the solar radiation problem, as they can only analyze $2 \mathrm{D}$ surfaces such as terrain and rooftops, with limited applicability to complex 3D urban surfaces (Hofierka \& Zlocha, 2012). This is due to the main limitation of the GIS solar radiation model, which is caused by using a stereographic projection of the sky, in which the whole sky is projected as a flat circle, which leads to inevitable errors caused by spatial distortion during the translation from 3D to 2D (Erdelyi et al., 2014). Also, when performing larger scale analysis at the block or postal code level, a loss in geospatial accuracy occurs (Resch et al., 2014). Note that when analysis is performed on DEMs of different resolutions, especially for complex scenarios, different estimations of elevation, slope, aspect and shadowing will be provided, which causes a loss in accuracy (Ruiz-Arias et al., 2009).

In summary, there exist many software tools and methodologies that have been developed for the assessment of rooftop PV potential. However, these methodologies lack the level of detail required for a comprehensive analysis of solar PV potential at the neighbourhood level. Hence, it is important to model the environment in a full 3D environment, and not just limited to the solar assessment of rooftops but to extend the analysis to facades $\left(90^{\circ}\right.$ vertical surfaces) and the surrounding environment as well. The accurate simulation of mutual shading between buildings in an urban environment is very important, as shading is one of the major loss mechanisms in PV electricity potential (Strzalka et al., 2012). This will lead to a better understanding of the area of interest and the various interactions between different objects (Catita et al., 2014). The understandings of these parameters are especially important for optimal solar neighbourhood planning.

\subsection{D Urban Rooftop and Façade Modeling}

Facades represent a great opportunity for increasing surface area available for PV deployment, as they comprise approximately $60 \%-80 \%$ of buildings surfaces in an urban environment (Esclapes et al., 2014). This far exceeds the available surface area on rooftops. Although vertical facades receive relatively less solar irradiation due to non-optimum inclination, the fact that there is more available surface area on facades then rooftops offsets this not so ideal quality (Catita et al., 2014). Especially in northern climates, where snow accumulation can be a 
potential problem for roof mounted solar PVs, facades represent a great solution to overcome this difficulty (Jochem et al., 2011). Another benefit for extending the analysis to vertical facades is that they will produce maximum power at different hours of the day, which will lead to a widening of the peak power production throughout the year (Redweik et al., 2013). For example, PV systems installed on rooftops would have higher output during the summer months, while facades would instead have peaks in spring and autumn (Romero Rodriguez et al., 2017b). It is for these reasons that it is important to extend analysis from rooftop solar potential to façades in a fully 3D modeling environment.

There have been some recent advances in the development of methodologies for the extension of solar potential assessment to vertical facades in urban environments. An early computer system developed to support solar access decision-making in a sustainable urban design perspective is called Townscope (Teller \& Azar, 2001). This software provided a 3D visual interface in an urban information system and is coupled with solar evaluation tools for solar community analysis of facades and rooftops. Another software tool called RADIANCE that is based upon ray tracing methods to identify the potential of facades and roofs in urban areas for PV potential have been used as well to study rooftop and façade solar availability (Compagnon, 2004).

Later studies performed by Robinson et al. (2006) focused on the development of a 3D city model for the modeling of solar potential on rooftops and facades of buildings.

The authors found that there was a need for a model that could handle the computational power and complexity required for the modeling of multiple buildings at once, and that could take into consideration a neighbourhood of about 50 - 500 buildings (Robinson et al., 2007). This led to the development of a program called SUNtool (sustainable urban neighbourhood modeling tool) for decision-making, design and optimization of sustainable neighbourhoods (Robinson et al., 2007). As time progressed, SUNtool became the predecessor to CitySim (Robinson et al., 2009).

CitySim is a 3D software tool for sustainable urban modeling that can handle a scale of analysis from a neighbourhood of just a few buildings to a district of several hundred to even an entire city. The modeling requirements can be summarized as follows: (1) site location and climate data, (2) choice and selection of default datasets for the type and age categories of buildings to be studied, (3) definition of the 3D form of buildings and building attributes, and (4) 
analysis. Using this software requires a certain level of user experience due to assignment of default values depending on the building and system design (Tereci et al., 2013).

Efforts in the development of methodologies and extending the solar analysis functionality of GIS based platforms to incorporate vertical facades have been performed by Jochem et al. (2011), Hofierka \& Zlocha (2012), Redweik et al. (2013), Catita et al. (2014), Erdelyi et al. (2014), Esclapes et al. (2014), and Takebayashi et al. (2015). These studies extend the functionality of the GIS solar modeling function by developing their own separate numerical algorithm based upon different sky-diffuse models to calculate the PV potential on facades. Takebayashi et al. (2015) tried to extend the rooftop analysis to facades by using only the solar radiation on the edge of the roof by reducing the virtual height of the building. Traditional GIS models are not capable of considering the facades of buildings since facades correspond to vertical discontinuities in the technically 2.5D DEM that they are based upon, as 2.5D models do not allow for the consideration of several heights in the same xy coordinate location (Redweik et al., 2013). Although many current GIS platforms provide 3D GIS capabilities to some extent, most of these capabilities are limited to visualization purposes only. Overall, there is a lack of adequate 3D capabilities for 3D solar applications (Hofierka \& Zlocha, 2012).

Romero Rodriguez et al. (2017a) used a 3D cityGML model in an urban simulation platform called SimStadt (developed at the University of Stuttgart) to perform a county wide regional scale assessment of rooftop PV electricity potential. The simulation methods were based on a building by building roof surface analysis and irradiance simulation and usage of reduction factors (rooftop, shading, PV) for the annual energy yield estimation. Due to the large amount of buildings in the study, only the geometry of the roofs were analyzed and the interaction between buildings for shadowing was not considered for shading. An Urban Shading Ratio function was added to SimStadt by Romero Rodriguez et al. (2017b), to calculate shadows on rooftops and facades, however only a resolution of $300 \mathrm{~m}$ minimum requirement to compute solar irradiance within an acceptable accuracy and to get a finer resolution estimate for such a large area was unfeasible computational efforts. Thus, for precise calculations, the more favourable option would be to use 3D modeling and building energy simulation software tools, however the main setback would be that it would only be able to handle small-scale regions (vs. larger scale regions from GIS) (Romero Rodriguez et al., 2017a; Romero Rodriguez et al., 2017b). 
There are numerous 3D industry and commercial based software tools that are capable of solar potential analysis at the rooftop and façade level. These software tools include PV*SOL, Skelion, Archelios, PVsyst, and Ecotect to name a few. Hachem et al. (2011) developed a methodology to investigate the solar potential of residential housing units in different neighbourhood designs using Google SketchUp and EnergyPlus. Analysis was performed on different neighbourhood layouts to investigate the differences in solar potential from the effect of parameters such as geometric shapes of individual units, density, site layout, and shape of rooftops. Similarly, Tereci et al. (2013) performed analysis at the residential community level, investigating the mutual shading effect between buildings and variations in urban form as to calculate the impact of urban form on energy demand using 3D CAD based model and EnergyPlus. Karteris et al. (2014) performed an urban scale analysis of facades for PV systems on residential multifamily buildings using SketchUp for CAD purposes, GIS for visualization, and EnergyPlus for solar potential calculation on the facades. Similarly, Sarralde et al. (2015) and Machete et al. (2018) performed 3D analysis on rooftops and facades at the neighbourhood level using a combination of the solar analysis functions in GIS and Ecotect Analysis.

This discussion has provided a summary of research studies that focus on the development of new methods and simulation techniques for the modeling of urban solar potential on rooftops and facades of buildings. These studies range in varying levels of complexity and expertise. The number of surface interactions and radiation exchanges also increase exponentially depending on the scale of the study which can present a significant impediment (Romero Rodriguez et al., 2017b). Hence, when conducting a study, it should be kept in mind the compromise between result accuracy and computational efficiency. There is a need for the evaluation of the physical functions and capabilities of the various software tools available to identify where their main discrepancies in estimation occur.

\subsection{Evaluation of Solar Simulation Software Tools}

There are numerous solar simulation software tools that are currently being used in the simulation community. Simulation programs have evolved over the past few decades from strictly text-based, programming languages to multiple interfaces for different outputs (Crawley et al., 2008). Differences in development history of the software tools, areas of focus, and levels of complexity results in differences in solar radiation estimation accuracy. This is primarily due 
to the techniques and algorithms that are used within the software tools (Chow et al., 2005). For the purposes of urban community simulation, it is important to achieve an adequate compromise between modeling accuracy, computational overheads and data availability (Robinson et al., 2009). There are several parameters that need to be considered when selecting a modeling software tool such as accuracy, sensitivity, computational time, cost, reproducibility, userfriendliness, level of detail of analysis, required data availability, and quality of the output (AlHomoud, 2001). The comparison can be difficult as there is no common language when describing the capabilities of different software (Li et al., 2014). Hence, it is important to be able to identify what each software tool can and cannot do, and the requirements.

Few studies have been performed on the detailed comparison between software capabilities and/or accuracy assessment of the solar simulation outputs. There are plenty of papers as mentioned in Sections 2.4 and 2.5 that focus on the development of new simulation techniques and models for the estimation of solar irradiance, or solar PV, however, little research has been performed regarding the calculative accuracy of commercial PV simulation software packages (Axaopoulos et al., 2014) or the comparison of the results from the individual submodels in the software tools. In addition, there continue to be underlying modeling gaps for system derates, emerging technologies, and characteristics of large systems (Freeman et al., 2013). Hence, this gap in the literature opens an opportunity for the thorough examination of the capabilities of these solar simulation software tools.

Crawley et al. (2005) performed an in-depth study contrasting the performance of twenty major building energy simulation programs. This study provided a good background for the various capabilities of building performance simulation software tools. The study included a brief overview of each program and a series of tables comparing the general modeling features such as zone loads, HVAC systems, building envelope, daylighting and solar, infiltration, ventilation, room air and multizone air flow, climate data availability, and links to other programs to name a few. The survey was very comprehensive, however, only the technical features of the software were examined and there was a lack of focus on the various solar functions of the simulation programs.

Yates \& Hibberd (2010) published an article on the production modeling for grid-tied PV systems which evaluated the performance of PV production modeling tools. The scope of the article was to look at the major software packages that were utilized by researchers, integrators 
and project developers in North America. The software packages that were reviewed in this study are: PVWatts, System Advisor Model, PV-DesignPro, PV*SOL and PVsyst. The study compared the POA irradiance calculations, as well as the electricity generation values. The conclusions of the research found that the radiation model components of the compared tools performed consistently, and the POA irradiance results were similar. In regard to electricity estimates, PVsyst presented the most conservative estimates and SAM the most aggressive, with an average difference of $9 \%$ between their estimates.

Lalwani et al. (2010) provided a review paper of twelve solar PV simulation software tools: RETScreen, PV F-Chart, SolarDesignTool, INSEL, TRNSYS, NREL Solar Advisor Model, ESP-r, PVsyst, SolarPro, PV-DesignPro-G, PV*SOL, and HOMER. The following information about each software tool were briefly discussed: (i) commercial availability and cost, (ii) working platform, (iii) working capacities, (iv) scope and outputs, and (v) updatability. A similar review study conducted by Sharma et al. (2014) was also performed on these solar simulation tools with the same review criteria as Lalwani et al. (2010). Both articles did not further elaborate on the functional capabilities of these software tools or provide any comparisons or analyses. The papers did not identify which tools are 2D or 3D and was not particularly helpful for identifying which simulation tool would be useful for 3D solar PV community energy analysis.

Cameron et al. (2011) performed a study comparing the variations in results in PV simulation in predicted energy output among modeling tools and users and compare that to actual measured values. Twenty different users were asked to perform a set of analyses for three systems along with recorded solar resource and weather data on whichever PV modeling platform or models they wanted. Seven different module performance algorithms were used: PVsyst, PVForm, PVWatts, the 5-parameter model from University of Wisconsin Solar Energy Laboratory, and the Sandia Array Performance Model. A wide range of results were obtained, even among those using the same modeling tools. Most of the predictions overestimated the measured results. The difference in results were attributed to uncertainty and variability in model inputs and the choice in derating factors chosen by the different users. Evidence suggests that uncertainty in model inputs causes many project developers to be conservative in their performance predictions, as many observers have reported that most systems have higher than expected performance. 
Lee et al. (2011) assessed the monthly and annual performance of four PV modeling software tools: PVsyst, HOMER, RETScreen, and SMA Sunny Design. This study was conducted using the data collected at the Desert Knowledge Australia Solar Centre solar technology facility. Five ground mounted PV systems on frames were analyzed, and one roofmounted PV system, totaling to six PV systems of various material (monocrystalline, polycrystalline, amorphous and $\mathrm{CdTe}$ ) under one type of sky model and array performance model. The results showed that all the PV modeling tools slightly underestimated the performance of the PV systems, which implies that the mathematical calculations tend to be conservative. All modeling tools had an average accuracy within 5\% of actual performance, and the order of accuracy from highest to lowest was found to be HOMER, RETScreen, PVsyst, and Sunny Design.

The International Energy Agency (IEA) Task 41 - Solar Energy and Architecture, conducted a review of solar design tools and methods from an architectural perspective (IEA, 2012). This study identified eighteen tools available for solar analysis and provided a brief overview of their capabilities. However, these software tools cover a broad spectrum of architectural functionalities and range from numerical outputs to daylighting analysis and are not all specific for 3D community energy modeling. Also, a performance/accuracy assessment of the outputs was not conducted in the study.

Axaopoulos et al. (2014) performed an accuracy analysis on six major PV estimation software tools: TRNSYS, Archelios, Polysun, PVsyst, PV*SOL, and PVGIS.

The analysis was done using actual climate data measured on site over the same calendar year, and by comparing the outputs of the software to the actual real electricity generated by a $19.8 \mathrm{~kW}_{\mathrm{p}} \mathrm{PV}$ park grid connected system. TRNSYS was found to be the most accurate software, and PVGIS to be the least accurate. The other software packages were generally found to overestimate global irradiation received by the PV modules but significantly underestimate the electrical energy generation of the installations. The main limitation of the study was that it only focused on the calculative accuracy of the software tools. The technical capabilities such as user interface, features and support were not discussed. Another limitation of this study was that it focused on the analysis of a PV park case study area, which does not take into consideration urban buildings and $90^{\circ}$ facades and neighbourhood building shading. 
Freeman et al. (2014) performed an analysis on nine PV systems using SAM, PVsyst, PV*SOL, and PVWatts. These tools were chosen due to their popularity. This study showed that all tools achieve annual errors within $\pm 8 \%$ and hourly root mean squared errors less than $7 \%$ for all systems. In particular, it showed that the SAM module model and irradiance input choices can change the annual error with respect to measured data by as much as $6.6 \%$ for these nine systems, although all combinations examined still fall within an annual error range of $\pm 8.5 \%$.

Freitas et al. (2015) performed a qualitative review of solar modeling tools in the urban environment ranging from 2D to 3D applications. Summaries of the different solar radiation model concepts were provided, highlighting the main descriptions of the software tools, however, no actual analyses and/or comparisons of the software tools were made.

In a study performed by Gurupira \& Rix (2017), three commonly used PV simulation software tools were compared: PVsyst, NREL SAM, and PVLib. The software tools were assessed against eight evaluation criteria: cost and commercial availability, working platforms, updatability, user-friendliness and ease of use, reporting and analysis options, modeling flexibility, performance and economic modeling capability, and validation of simulation results with field data. The authors found that all three tools underestimated the total system yield, and this is mainly attributable to the overestimation of losses in the systems default loss models. PVsyst was the most accurate with a 3.4\% difference, followed by SAM with $3.9 \%$ difference, and lastly PVLib with $5.1 \%$.

Jakica (2018) presented a study that provided an overview of approximately fifty solar simulation and design tools from various interdisciplinary fields. Disciplines that were looked at included daylighting, whole building energy, optical design, visualization/rendering (still movies/animation), gaming (real time interactive graphics) and PV/BIPV. Features such as accuracy, complexity, scale, computational speed, representation and building design process integration were analyzed in the paper in a broad multidisciplinary context for solar design with particular emphasis on Daylighting and spectral ray modeling purposes. The author provided a high-level overview of solar design tools and methods, however no actual analyses with real data or simulations were performed. The paper was focused on a broad overview of all solar tools available in the market (ray tracing, daylighting, etc), and was not focused specifically on 3D solar PV modeling. 


\subsection{Summary of Solar Software Tools}

There are a lot of solar simulation software tools that are out there in the world. Different software tools were created for different reasons and have many different features and capabilities that are suitable for certain tasks/projects over others. Due to the vast amount of software tools out there, this section will be providing a short description on the various tools that were identified in the literature review. This is in no way a comprehensive list of every tool available in the market. The purpose is to highlight the most commonly used software tools in research and industry based upon the literature review. This summary will be categorized into these main topics: (1) GIS based models, (2) open source software, and (3) commercial software tools.

\subsubsection{GIS based models}

\section{(i) PVGIS}

PVGIS is developed by the European Commission and is available for free to perform PV potential analysis anywhere in the world. It is an interactive map and web interface that allows you to select your location from anywhere on the map, and to determine the solar radiation and PV performance of your project. Most of the solar radiation data used by PVGIS are derived from satellite images (PVGIS, 2020).

\section{(ii) ArcGIS Solar Analyst}

The solar radiation analysis tools in ArcGIS under the Spatial Analyst license analyzes and maps the effects of the sun over a specific geographic location over a specified time range. It is commonly used to determine the solar and rooftop availability for PV applications. The two main functions used are the Area Solar Radiation tool (which is used to calculate the insolation across an entire landscape) and the Points Solar Radiation tool which is used to calculate the amount of radiant energy for a given location (specific x,y coordinate location).

\section{(iii) r.sun}

The r.sun model is a solar radiation modeling module that is available in the open source environment Geographical Resources Analysis Support System (GRASS) GIS developed by Hofierka \& Suri (2002). R.sun is a raster-based mapping program for the modeling and mapping 
of complex terrain on horizontal and inclined surfaces and is especially applicable for modeling large surface areas. It requires expertise programming skills and is run in C programming language.

\subsubsection{Opensource software tools}

(i) EnergyPlus

EnergyPlus is a building energy simulation software program for modeling building heating, cooling, lighting, ventilation and other energy flows. It was developed by the United States Department of Energy and receives inputs and outputs as text files as a command line interface. EnergyPlus is a modular structured software tool that acts primarily as a building energy simulation engine (Crawley et al., 2005).

\section{(ii) $E S P-r$}

ESP-r was developed at the University of Strathclyde over 30 years ago and is an open-source software tool for the modeling of building performance simulation. It is primarily used as a research tool and is geared towards the modeling of heat, air moisture, light and electrical power flows for total building energy performance. It does not have a user-friendly interface and requires specialist skills in programming (ESRU, n.d.).

\section{(iii) PVLib}

PVLib is an open-source toolbox developed at Sandia National Laboratories that provides a set of well documented functions and classes for modeling solar energy and simulating the performance of PV energy systems. It provides models for reference implementations and algorithms such as solar position, clear sky irradiance, irradiance transposition, DC power, and DC to AC power conversion (Holmgren et al., 2018). PVLib has no graphical user interface and is strictly a script-based simulation tool. PVLib uses code-level modular programming to perform the modeling of the PV system and is available in Matlab and Python versions. PVLib requires high level user expertise, and hence mostly attracts users from academia and research (Gurupira \& Rix, 2017). 


\section{(iv) System Advisor Model (SAM)}

SAM was developed by NREL in collaboration with Sandia National Laboratories in 2005, and is a free techno-economic desktop software program that facilitates decision-making in the renewable energy industry (SAM NREL, 2020). SAM is a free software tool and it contains a broad set of models and frameworks for performing detailed analysis of both system performance and financial analysis. Its first public release was launched in 2007. SAM is an advanced modeling tool and calculates PV system performance using mathematical submodels developed by governmental and academic organizations such as NREL, Sandia National Laboratories, the University of Wisconsin and others (Hren, 2015). SAM was originally released as the "Solar Advisor Model", however as more models were added each year to SAM, the name was changed to "System Advisor Model" to incorporate the addition of other renewable energy technologies.

\section{(v) PVWatts}

PVWatts was created by the National Renewable Energy Laboratory (NREL), and it is an interactive application and map that allows for the basic solar modeling of hourly or monthly PV solar production based on minimal inputs. PVWatts "estimates the energy production and cost of energy of grid-connected PV energy systems throughout the world. It allows homeowners, small building owners, installers and manufacturers to easily develop estimates of the performance of potential PV installations" ( NREL, 2020). PVWatts is a cloud-based software platform and it is one of NREL's most highly trafficked websites with over 20,000 users per month (Hren, 2015). PVWatts is primarily used in residential home PV system estimates or as a preliminary assessment tool for the feasibility of larger systems.

\subsubsection{Commercial Software tools}

\section{(i) Archelios}

Archelios is a PV sizing software that was developed in 2001 at the University of Savoie, France. Archelios is available commercially and is currently distributed by Trace software. It is an online application and uses a SketchUp plugin available for 3D shading analysis and simulation. It is a decision support tool, and allows for the design, simulation and economic analysis of PV projects. 


\section{(ii) Skelion}

Skelion is a SketchUp plugin for the 3D modeling and analysis of solar PV potential. It allows the user to quickly add solar PV installations to their 3D model and has a PV component database. Skelion calculates yearly shading losses ratio that the PV system receives and then it communicates with the PV calculators of PVWatts and PVGIS to produce an energy report (Skelion, n.d.). It does not do hourly analysis on its own.

\section{(iii) RETScreen}

RETScreen is a Clean Energy Management Software system for the modeling of renewable energy and cogeneration project feasibility analysis and is developed by Natural Resources Canada (NRCan). It is a spreadsheet-based software and can help analyze and optimize the energy production, technical and viability of a project (NRCan, 2019).

\section{(iv) HOMER Energy}

HOMER (Hybrid Optimization of Multiple Energy Resources) is an energy modeling software tool that was developed at the U.S. National Renewable Energy Laboratory (NREL). It is a software tool that contains many different models for the simulation of various renewable energy technologies and optimization and economic analysis functions (HOMER Energy, 2018).

\section{(v) TRNSYS}

TRNSYS (Transient System Simulation Tool) is a commercially available building energy simulation software tool that has been available for over 30 years. It is used primarily for the simulation the behaviour of building energy demands, HVAC equipment, thermal and electrical energy systems, renewables and has an extensive library of components for building energy modeling (TRNSYS, 2019).

\section{(vi) Ecotect Analysis}

Ecotect Analysis is an Autodesk CAD based software tool that was designed for the estimation and performance of solar energy potential analysis for buildings in an urban environment. It has a variety of performance analysis functions that are capable of handling the visualization, 
thermal performance, solar energy analysis, sun-path diagrams, shading, ventilation, environmental impacts and cost aspects of the simulation process (Marsh 2003; Crawley et al. 2008). The software is discontinued and no longer available as of 2015.

(vii) Aurora Solar

Aurora Solar is a web (cloud)-based optimization platform that automates the design, engineering and permit generation process of solar PV projects and installations (Freeman \& Simon, 2015). Users can generate rooftop drawings over a satellite image and design their PV system from the actual geometry of the rooftop and surrounding obstructions. It is a sales and design software for project installations and is not available for academic/student usage.

\section{(viii) Helioscope}

Helioscope is a commercial web/cloud-based PV system modeling tool that was released in 2014 by Folsom Labs. It is a design and engineering application that enables developers and engineers to simulate the performance of PV systems on an annual and hourly basis. It allows users to create fast and easy to use designs with features such as automatic module layout, in-browser 3D shading analysis, and cost-benefit comparisons. Helioscope has a graphical user interface that is online and uses Google Maps and Bing Maps for the project location. Its target audience are project developers, engineers, sales technicians and designers.

\section{(ix) PVsyst}

PVsyst was founded and developed by Andre Mermoud from the Univeristy of Geneva in 1992. PVsyst is a PC software package for the study, sizing and data analysis of complete PV systems. PVsyst is designed for architects, engineers and researchers, and it deals with grid-connected, stand-alone, pumping and DC-grid PV systems, and includes extensive meteorological and PV systems components databases, along with general solar energy tools (PVsyst, 2020). It has a detailed help menu that provides thorough explanations of the underlying models, theories and procedures that are used in order to provide a user-friendly approach for simulations. 


\subsection{Benchmarking}

The word "benchmarking" is a popularly used term, with applicability in many different fields with many opinions on what it is, why it is necessary, how it should be done, and what results to obtain (Rolstadas, 1995). The benchmarking term has been widely used to refer to many different activities and some of the main definitions that were come across in the literature are:

- According to the Merriam-Webster dictionary, the term benchmark is defined as "something that can be used as a way to judge the quality or level of other, similar things"

- According to the Oxford Dictionary, it is "a standard or point of reference against which things may be compared"

- According to the International Standard ISO/IEC/IEEE 24765 (2010) Systems and Software Engineering Vocabulary, the term benchmark is officially defined as "1) $a$ standard against which measurements or comparisons can be made, 2) a procedure, problem, or test that can be used to compare systems or components to each other or to a standard, and 3) a recovery file"

- "A test or set of tests used to compare the performance of alternative tools or techniques" (Sim et al., 2003).

Over the past few decades, benchmarks have proven to be useful to demonstrate performance competitiveness for products and to improve/monitor performance of products or technologies (Nambiar et al., 2009). Software tools need to be benchmarked because there is a need to assess the quality of them and the overall usefulness. A good benchmark provides a means for a level playing ground for competing ideas, allows repeatable and objective comparisons, and eliminates unpromising approaches (Tichy, 1998). Evaluating competing technologies on a common problem set is a powerful way to improve the state of the art and hasten technology transfer (Voorhees, 2005). Benchmarks have been utilized in many differing fields of research, such as speech understanding, information retrieval, pattern recognition, software reuse, computer architecture, performance evaluation, applied numerical analysis, algorithms, data compression, logic synthesis, and robotics (Tichy, 1998). In general, the community of interest may include those from academia, industry, and government for scientific research. Benchmarks can range 
from very simple that target a specific system, to very complex ones for complicated infrastructures and systems (Viera \& Madiera, 2009).

Benchmarking is an empirical method. It has characteristics from both case studies and experiments and shares features from both of these empirical methods. Table 1 summarises the comparison of benchmarking as an empirical method to experiments and case studies (Sim et al., 2003).

Table 1. Comparison of Benchmarking as an Empirical Method to Experiments and Case Studies (adopted from Sim et al., 2003)

\begin{tabular}{|c|c|c|c|}
\hline & Features & Advantages & Disadvantages \\
\hline $\begin{array}{l}\text { Characteristics } \\
\text { from Experiments }\end{array}$ & $\begin{array}{ll}- & \text { Use of } \\
& \text { control } \\
& \text { factors } \\
\text { - } & \text { Replication } \\
\text { - } & \text { direct } \\
& \text { comparison } \\
\text { of results }\end{array}$ & $\begin{array}{ll}\text { - } & \text { Direct } \\
& \text { comparison } \\
\text { of results }\end{array}$ & $\begin{array}{l}\text { - Not suitable } \\
\text { for building } \\
\text { explanatory } \\
\text { theories }\end{array}$ \\
\hline $\begin{array}{l}\text { Characteristics } \\
\text { from Case Studies }\end{array}$ & $\begin{array}{ll}\text { Little } \\
\text { control } \\
\text { over the } \\
\text { evaluation } \\
\text { setting (eg. } \\
\text { choice of } \\
\text { technology } \\
\text { and user } \\
\text { subjects) } \\
\text { - No tests of } \\
\text { statistical } \\
\text { significance } \\
\text { Some open- } \\
\text { ended } \\
\text { questions } \\
\text { possible }\end{array}$ & $\begin{array}{l}\text { - } \text { Method is } \\
\text { flexible } \\
\text { and robust }\end{array}$ & $\begin{array}{ll}- & \text { Limited } \\
\text { control reduces } \\
\text { generalizability } \\
\text { of results }\end{array}$ \\
\hline
\end{tabular}

As an experimental approach, its acceptability is based on the ability to reproduce the observations and the measurements, and the capability of generalizing the results through some form of inductive reasoning. These provide confidence in the results and the generalizability makes the results meaningful and useful beyond the specific setup used in the benchmarking process (Vieira \& Madeira, 2009).

Furthermore, benchmarking can help to identify weak points and critical issues, opportunities and areas for improvement, and to plan actions directed to achieve these improvements (Ukovich \& Zerilli, 1995). Benchmarking provides a public evaluation which contrasts with the 
descriptions of tools and techniques and their features and capabilities. It makes it difficult to hide the flaws of a tool or technique or to exaggerate the strengths, as this provides a transparent way of testing. Benchmarking results should be reproducible with the same tools/techniques (Sim et al., 2003).

To develop an approach to software benchmark design, it is important to know the interest in which why the benchmark is being performed. For example, a marketing company would perform a benchmark in order to determine the marketing power of its software products (Daneva, 1995). Frequently asked questions regarding performance benchmarking typically include: What should I measure? What units of measurement have other successfully used? With whom should I compare? Who should do the measuring? (Eloranta \& Crom, 1995). What are used as points of reference for a benchmark depends on the reason or objective for benchmarking (Eloranta \& Crom, 1995).

Huppler (2009) introduces 5 key aspects that make a benchmark good and they are:

(1) Relevant - the benchmark reflects something important to the reader of the results

(2) Repeatable - the benchmark can be run again with the same results

(3) Fair - all systems and/or software being compared can participate equally

(4) Verifiable - confidence that the results are real

(5) Economical - the benchmark can be run at an affordable cost A good benchmark that has longevity should include all 5 of these elements, though not necessarily in equal weights.

Maneva et al. (1995) suggested the following 6 step procedure for benchmarking: (1) state the goal, (2) define the relevant objects and their attributes, (3) define the set of competitive objects and compare them, (4) plan and accomplish the appropriate course of actions, (5) measure results against goal, and (6) re-evaluate and continue.

Since innovations in the computing and software industry are always evolving, appropriate representation is very important to ensure that the benchmark is not misused to represent something that was not intended, and that the benchmark can be developed within a reasonable time frame (Huppler, 2009). 


\section{RESEARCH OBJECTIVES}

This chapter will be discussing the objectives of this thesis, research questions and the motivation behind the research.

\subsection{Thesis Objective}

The objective of this thesis is to increase the assessment ability of solar energy utilization and planning support for clusters of different types of buildings in a mixed-use community. Particular focus will be placed on the analysis of community-based modeling, mapping and forecasting of solar potentials on the rooftops and facades of buildings. New systems and methodologies with appropriate level of detail at a lower computational time, are needed to accurately model, estimate and map solar energy potential at a high spatiotemporal resolution. In order to accomplish this goal and to develop an integrated solution, solar potential was analyzed using two different types of studies: (1) 3D GIS modeling of a solar energy community, and (2) benchmarking of solar PV radiation software tools.

\section{3D GIS modeling and mapping of solar energy community}

GIS are widely used for the modeling and mapping of solar energy potential. GIS has large database storage capabilities and the ability to monitor both time and space. Since itcan determine exactly when and where a specific event is occurring, it was of research interest to explore the 3D capabilities of GIS for solar potential analysis. The objective of this part of the thesis was on the development of a 3D GIS modeling and mapping approach at a fine spatiotemporal resolution to assess community-based solar potential.

\section{Benchmarking of solar simulation software tools}

There are numerous solar simulation software tools that are currently being used in the simulation community. Simulation programs have evolved over the past few decades from strictly text-based, programming languages to highly visually appealing interfaces with various different outputs. Parameters such as accuracy, functionality, computational time, cost, reproducibility, user-friendliness, level of detail of analysis and quality of the output need to be considered when selecting a modeling platform. An assessment of these software tools is 
important to evaluate their strengths and deficiencies for performing analysis in different stages of the PV modeling process. The performance of the software tools will be assessed based upon their accuracy in simulation performance analysis and the comparison of their physical capabilities and functions. A case study area with an existing PV installation will be analyzed. Based upon the evaluation of these software tools, recommendations for the software tools would be made suitable for 3D community energy analysis.

Note that although there are many environmental and financial benefits associated with PV energy, the scope of this dissertation will be limited to the modeling and simulation of PV solar energy potential. Issues such as environmental modeling, life cycle analysis, and other financial/economical modeling aspects will not be discussed in this thesis, however are important factors to be considered for future works.

\subsection{Research Questions}

In order to reach the goals of this thesis, some research questions that needed to be addressed include:

- What climatic data is available?

- What are GIS's capabilities for 3D solar energy modeling and mapping?

- What are the available solar software tools for 3D modeling?

- What are the functions and capabilities of these 3D PV modeling software tools?

- What are the models used in these software tools for calculation? For example: solar radiation models? PV models? Inherent assumptions behind the software tools? Causes in differences of outputs?

- How accurate are these software tools?

- Feasibility for usage in analyzing solar energy communities? Pros and cons?

- Which software tools should be recommended?

\subsection{Research Motivation}

The knowledge of when and how much energy is used is critical to the management of local energy networks (Berry et al., 2014). The load on the electricity grid fluctuates daily and 
seasonally and can peak during extreme climatic events (Berry et al., 2014). These peaks can cause interruptions to the electricity supply and become problematic. The electricity system in Ontario has undergone significant changes in the past ten years, transitioning from a winter peaking one (due to building heating) to a summer peaking situation (due to building cooling). This change in pattern is mainly due to the increased demand for air conditioning in the summertime, and a decrease in the usage of electricity for heating in the wintertime (Brown \& Rowlands, 2009).

With the increase in PV development, and the integration into the electricity generation distribution networks, PV has added benefits to the electricity system by reducing transportation and distribution losses, improvements in quality and continuity of service during on-peak hours (Caamano-Martin, 2008). This is particularly important as markets are increasingly placing a higher value on electricity that is available at peak demand times in congested areas due to the associated costs of transmission (Kemery et al., 2012). A potential medium to long-term benefit of PV electricity is that it defers future investments to increase grid capacity and reduces the additional power generation needed to meet peak demand periods (Caamano-Martin, 2008).

Since PV power generation coincides with the summer cooling peak period, PV electricity can act as a distributed electrical generation source in urban areas and can help reduce dependence on the distribution and transmission systems (where blackouts are a high probability) due to excessive transmission loads (Nguyen et al., 2012). Solar communities can have the potential to help alleviate this problem by reducing peak load or lowering generation peaks for different times of the day in which congestion is at its highest. The end goal is to optimize the net electricity supply/demand to and from the grid to obtain a zero-peak community.

\section{METHODOLOGY}

This chapter will be discussing the methodology approach for this thesis. The organization of this chapter will be divided into two main sections:

1. Usage of GIS to model and map solar energy potential on a large-scale community level

2. Benchmarking of $3 \mathrm{D}$ solar PV simulation software tools

a. Performance analysis against measured data

b. Physical functions and capabilities of the software tools 
Figure 3 provides a depiction of the overall workflow that was employed in this thesis.

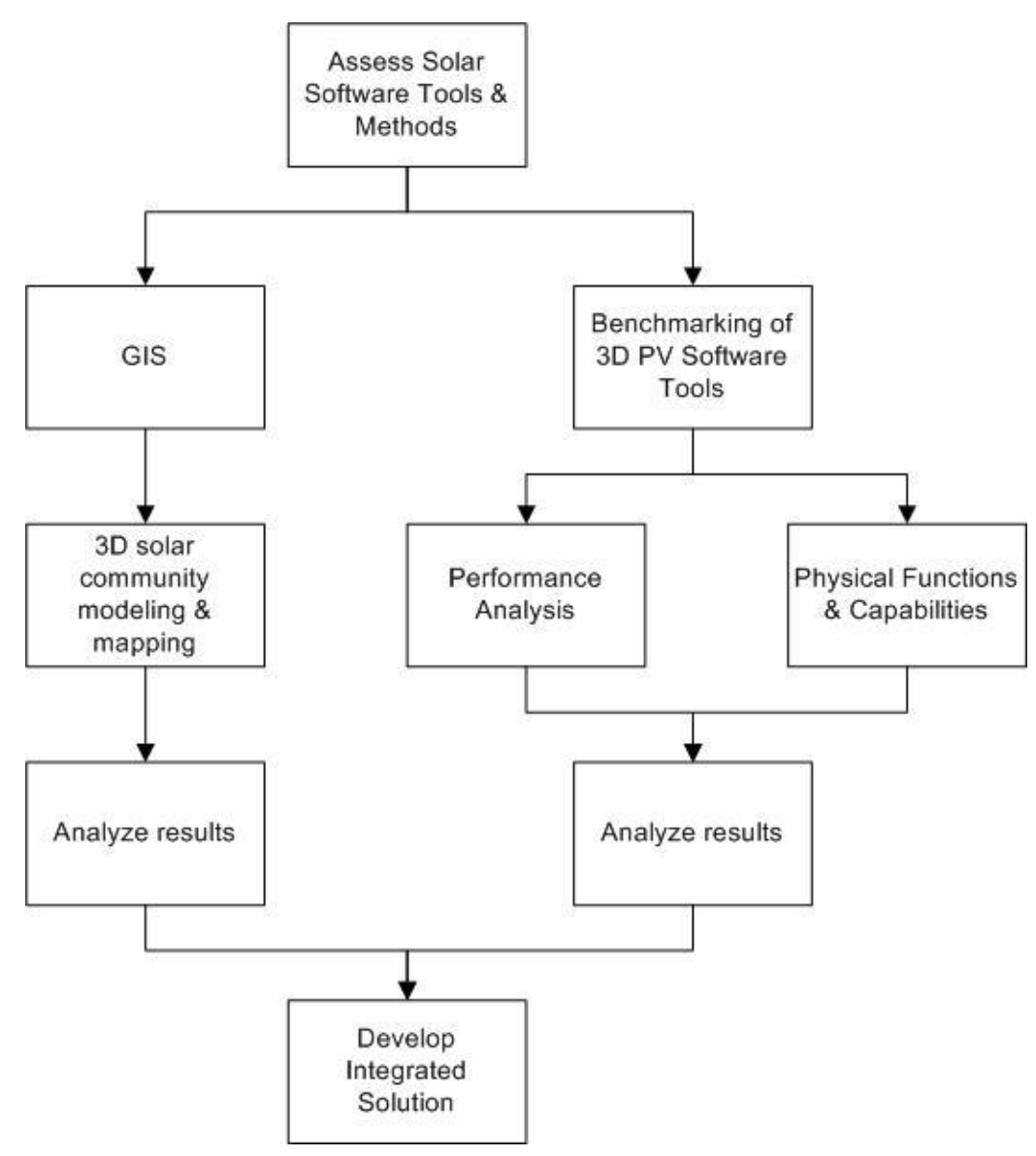

Figure 3. Overall workflow for dissertation.

\subsection{GIS Modeling of Solar Neighbourhood at a High Spatiotemporal Resolution}

This section of the thesis focuses on the development of a 3D GIS modeling approach at a fine spatiotemporal resolution to assess community-based solar energy potential. This research was published in Chow et al. (2014). GIS are particularly useful for such solar applications, as it has the ability to monitor both time and space and can determine exactly when a certain event or activity occurs (Longley et al., 2005). A model was created in ArcGIS 10.2, in order to efficiently compute and iterate the hourly solar modeling and mapping process over a simulated 
year. The Solar Analyst tool in ArcGIS allows for the modeling, mapping and analysis of solar insolation over a geographic area for specific time frames. It takes into consideration the effects from the atmosphere, latitude, elevation, inclination of slope, orientation, daily and seasonal shifts of the sun, and effects of shadows cast by surrounding topography and buildings.

A case study area for a future potential smart net-zero energy community was selected for analysis located in southern Ontario, Canada, in collaboration with our industry partner S2E Technologies Inc, through a Mitacs research internship. This site is approximately 70 acres in size and is to be the location of a multi-use community that will be comprised of 2000 living units (high rise, medium and townhomes), 100,000 square feet of office space and 400,000 square feet of retail/commercial space. A model of the site plan was obtained from the developers of the proposed solar community layout. Two situations were modeled: (i) original site plan as proposed by the developer, and (ii) alternative solar community design; in order to determine which community model was better suited for net-zero energy.

The 3D GIS modeling approach that was developed for the analyses of community based solar potential at a fine resolution was comprised of three main steps: (1) building a 3D case study model using high resolution orthophotos and CAD data; (2) estimation of solar potential; and (3) extraction of data. This methodology was tested on a case study area under two different 3D solar community design scenarios. This methodology is transferrable amongst any location provided the data are available, and Figure 4 provides a diagram of the overall workflow process. 


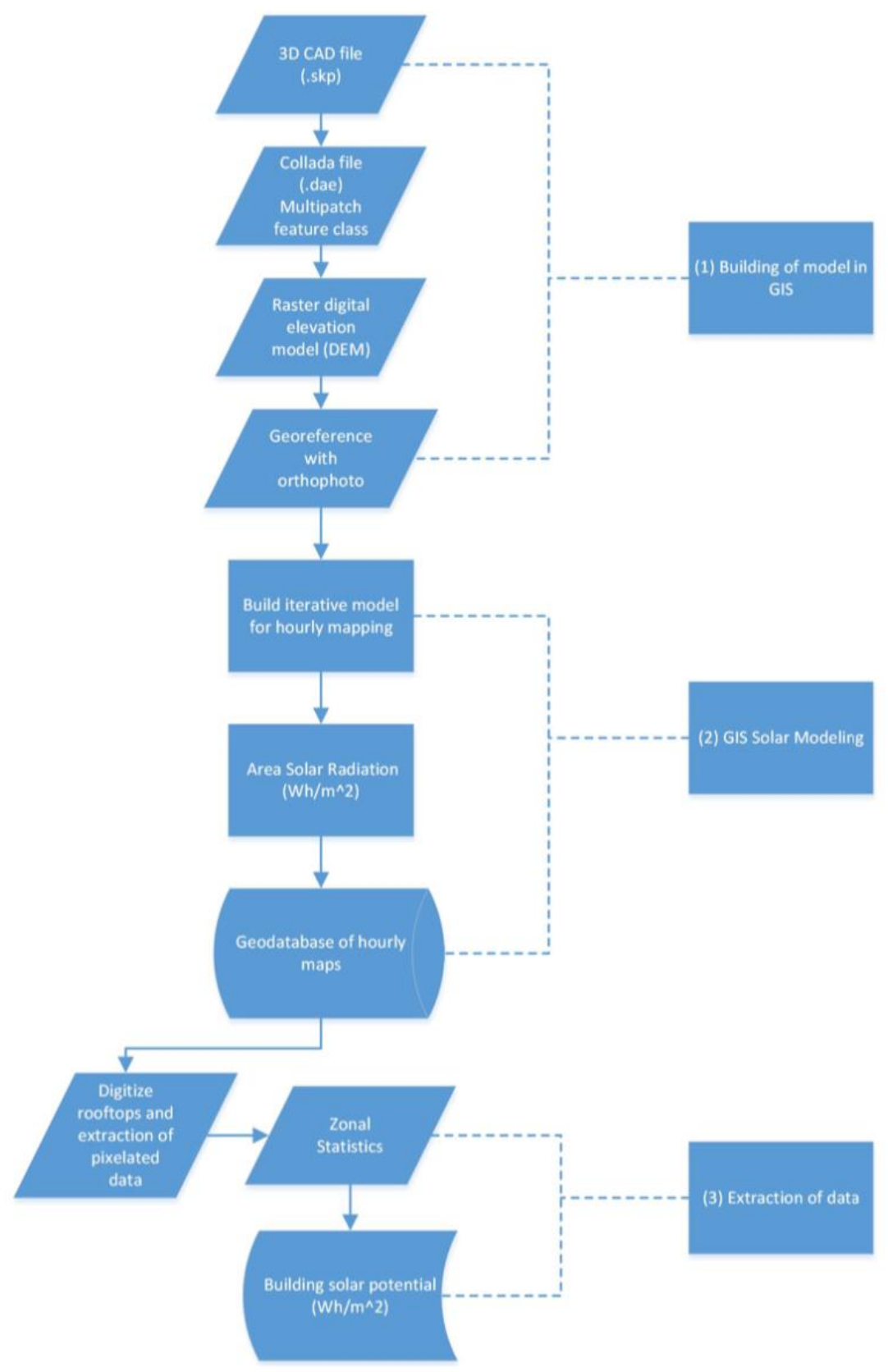

Figure 4. Methodology workflow for 3D GIS solar modeling of hourly solar potential 


\subsubsection{Building of the 3D Model}

Solar modeling functions in GIS require data that is spatially referenced with detailed elevation information in raster data format (pixelated data in the format of grids). 3D data of the original case study area was provided by industry partner in 3D CAD format which was in Google SketchUp (.skp) format. This data format was by default not readable by GIS. The 3D data needed to be converted into a 2D raster data digital elevation model (DEM) format in order to perform the solar analysis. This was done by importing and editing the SketchUp file into Collada (.dae) file format. The Collada file (multipatch feature class) was then imported into ArcScene, which displayed the corrected 3D file in GIS. An image of the 3D file created in ArcScene is illustrated in Figure 5.

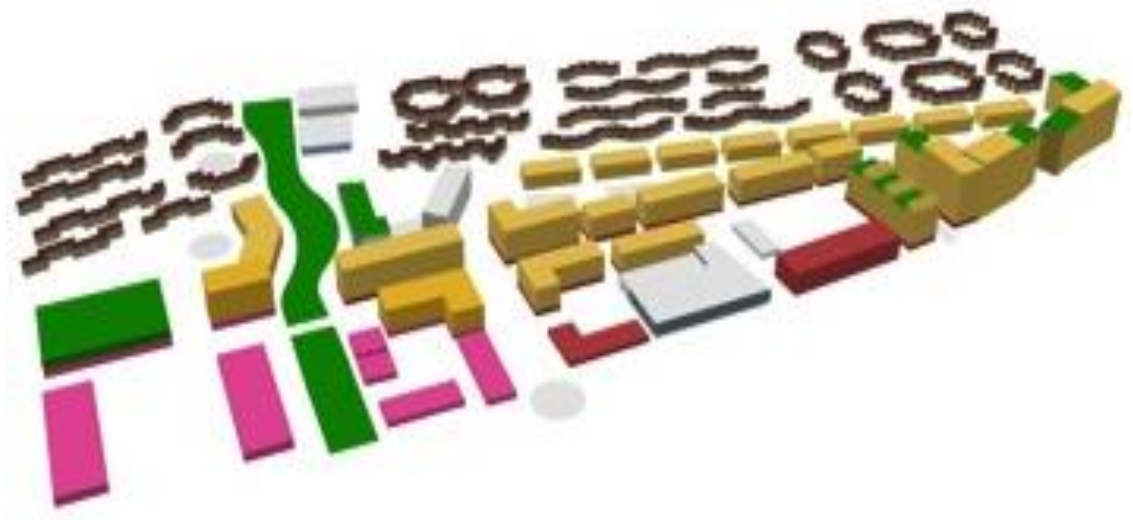

Figure 5. 3D model imported into ArcScene in .dae format.

The multipatch feature class was then converted into DEM format at different test resolutions of $0.05 \mathrm{~m}, 0.1 \mathrm{~m}, 0.5 \mathrm{~m}$, and $1 \mathrm{~m}$. A final pixelated resolution of $0.5 \mathrm{~m}$ was decided upon for the final analysis based upon computational time requirements and resolution tradeoff for solar mapping (which will be explained in the following section). The boundary of the street block was digitized to serve as a base for georeferencing. The obtained orthophoto of the case study area was acquired at a $15 \mathrm{~cm}$ resolution, from April 2013. The orthophoto was added to the DEM and georeferenced to the correct geographic coordinates of $42^{\circ} 57^{\prime} 59.71^{\prime \prime} \mathrm{N}$ and $81^{\circ} 21^{\prime} 38.28^{\prime \prime} \mathrm{W}$. Figure 6 and Figure 7 are images of the created DEM for the original site plan and alternative design plan respectively, from the original 3D CAD file obtained. 


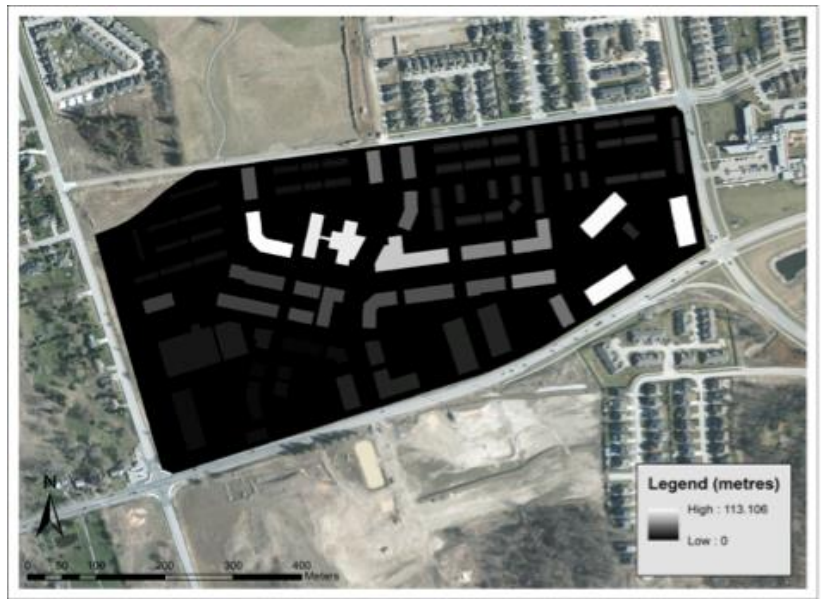

Figure 6. Original site plan design-converted $2 D$ raster digital elevation model (DEM) at a resolution of $0.5 \mathrm{~m}$ converted from $3 D$ file, projected onto orthophoto.

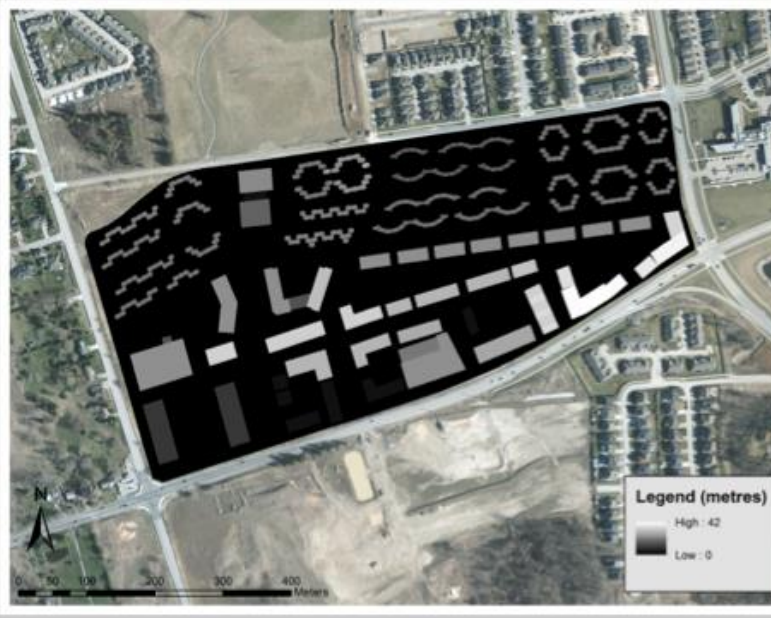

(a)

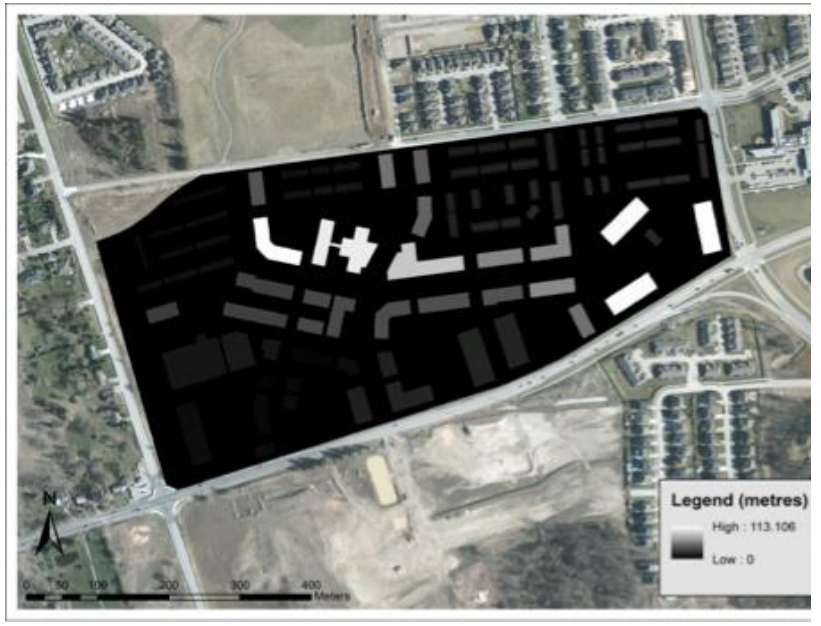

(b)

Figure 7. Converted $2 D$ raster DEM at a resolution of $0.5 \mathrm{~m}$ converted from $3 D$ file, projected onto orthophoto: (a) alternative community design, and (b) original case study design.

\subsubsection{GIS Solar Modeling}

Solar mapping the different configurations of the 3D model is beneficial for determining how hourly solar potential will be impacted by different design factors, as well as to establish which configurations are ideal for a net-zero solar community. Mapping the study area on an hourly basis will help with the determination of areas with maximum solar radiation exposure, to help with PV installation purposes. 
The solar tools in GIS analyze the effects of the sun over a specific geographic location over a range of time intervals. The Solar Analysis tools in ArcGIS 10.2 allows for one to take into account how the daily and seasonal shifts of the sun angle, elevation, orientation (slope and aspect), and shadows from surrounding features affect the amount of solar radiation received on any particular surface (in $\mathrm{Wh} / \mathrm{m}^{2}$ ) (ESRI, 2012a). In order to perform the analysis, an input 2D raster representation (DEM) of the case study area was created as discussed in the previous section, which contained detailed elevation information for the entire site. This model was imported into ArcMap 10.2, and the Area Solar Radiation tool was used to model the incoming solar radiation on the case study area. Area Solar Radiation is an extension of the Spatial Analyst tool in ESRI ArcGIS. It is capable of performing calculations on complex rooftop shapes, as it takes topographic constraints from the model into account.

The Area Solar Radiation tool calculates the amount of insolation received across an entire landscape at a specific location based on the hemispherical viewshed algorithm (ESRI, 2012b). The hemispherical viewshed approach is used to assess sky obstructions for any location on or above a topographic surface. It allows for the performance of rapid calculations and permits users the control over the level of resolution to use in the simulations (Rich et al., 1994; Fu \& Rich, 1999). The viewshed method is used together with sun position and sky direction information for the calculation of direct, diffuse and total solar radiation for each specified location to calculate an accurate solar insolation map. The main input parameters and their descriptions for the Area Solar Radiation tool are:

- Latitude - Coordinate of location

- Sky size resolution - Resolution/sky size of viewshed model

- Day interval - Time interval through the year in days

- Hour interval - Time interval in hours used for calculation (can go up to 0.5 hours)

- Time configuration - Specify the period of time to perform the calculation

- Z-factor - The number of ground $\mathrm{x}, \mathrm{y}$ units in respect to $\mathrm{z}$ axis

- Slope/aspect - Configuration of slope/aspect from inputted dataset or to override values to 0

- Calculation directions - Number of azimuth directions used to calculate viewshed

- Zenith and azimuth divisions - Number of divisions used to create sky sectors in map 
- Diffuse radiation model - Uniform sky model, standard overcast sky model for calculation

- Diffuse proportion - Ranges from 0 to 1 . Proportion of global normal radiation flux that is diffuse

- Transmittivity - Fraction of radiation that passes through the atmosphere (ESRI, 2012c).

Solar modeling in GIS is a very intensive geoprocessing (computational time) process and can range from a few hours up to multiple days for the performance of one simulation on a large data set for only a single hour time frame. In general, the finer the resolution of the sky size and raster input map, the more accurate and visually appealing the results will be. However, this will cause simulation time to increase exponentially. An optimal solution for the trade-off between resolution, time-interval and computational time was needed. Thus, the Area Solar Radiation tool was tested on many different time intervals and resolutions in order to find an optimal solution within a reasonable computational time period. A regular desktop computer was selected for analysis, as most professionals do not have access to a super computer in their office, and this methodology was intended for an average user to be able to replicate.

At the time of the analysis, a desktop computer was used with a 6 generation Intel i76700 4-core (8-thread) 3.4GHz CPU with 32GB RAM running a 64-bit Windows 8 Pro. This computer represented an average computer that would be available to a general industry worker, without the need for the usage of a supercomputer (as that would not be readably accessible). The computational time periods tested ranged from multiple hours to days for one analysis due to the scale of the community model and the complexity of the design of the buildings. Resolutions of $0.05 \mathrm{~m}, 0.1 \mathrm{~m}, 0.5 \mathrm{~m}$ and $1 \mathrm{~m}$ were tested with various parameter inputs for time efficiency. The size of the community analyzed was over 70 acres in size, and comprised of over 2000 residential homes (townhomes, apartments, condos), 400,000 square feet of retail space and 100,000 square feet of office space. Taking into consideration the size of the case study area, with the typical townhome building footprint having an approximate surface area of 645 square feet and retail building of 8000 square feet, there was a lot of surface area to be considered for installation. Typical residential systems usually use 60 cell PV modules while commercial systems use 72 cells or more for higher outputs. A typical 60 cell polycrystalline PV module measures $1.6 \mathrm{~m}$ by $1 \mathrm{~m}$ while a typical 72 cell module measures $2 \mathrm{~m}$ by $1 \mathrm{~m}$. The total dimension of 
the grid that was analyzed (including the surrounding area for the community) was $1167 \mathrm{~m}$ by $817 \mathrm{~m}$. At $0.05 \mathrm{~m}$ resolution, the computer was not able to simulate the large case study area and would crash after approximately 4 to 6 hours. At $0.1 \mathrm{~m}$ resolution, the computer would simulate for approximately 24 to 30 hours, before crashing due to the size of the grid. The next resolution selected was $0.5 \mathrm{~m}$ and it successfully simulated the solar maps and took approximately 336 hours ( 2 weeks) to perform the hourly analysis over the simulated year for one scenario of the case study area. At the 1 metre resolution, the annual simulation per scenario of the case study area took approximately 72 hours to perform which generated a map for every daylight hour of the year. After testing the processing time at these various resolutions, a final output resolution of $0.5 \mathrm{~m}$ was chosen.

The Area Solar Radiation function cannot automatically simulate and map hourly values over an annual basis. In order to overcome this setback, a customized model was built in GIS to automatically iterate the hourly simulations. The Model Builder software tool was used to create an iterative hourly model. Model Builder graphically depicts the processing steps and represents datasets as ellipses, operations as rectangles, and the sequence of the model as arrows. This allows for an interactive graphic display, and control over the operation sequences (Longley et al., 2005). Note that this can also be accomplished through Python scripting, since almost any model in GIS can be expressed as a script or visually as a flowchart. This model was used to iterate the hourly simulation process, performed in batches, one month at a time for a simulated year. The model that was created and used in this research project is illustrated in Figure 8. An example of the input parameters for the month of January can be found in Appendix A. 


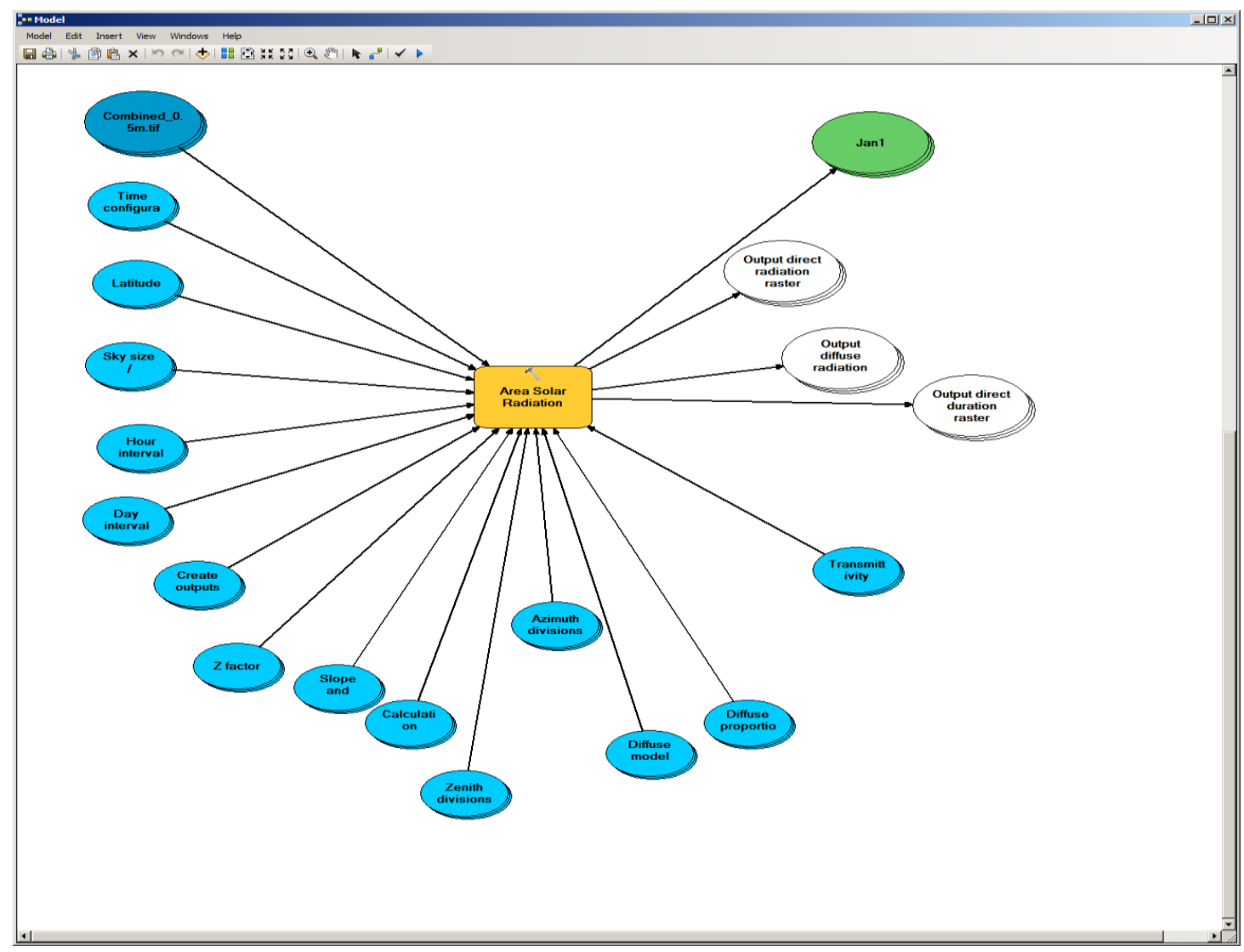

Figure 8. Graphic representation of the Area Solar Radiation Model for iterative analysis.

Raster data sets were generated for each day of the simulated year, in which solar analysis outputs and hourly maps were stored. This output was generated in a GRID stack format, which contained multiple bands containing values corresponding to the time configuration of the analysis. The solar maps were extracted from the bands in the GRID stack format, and the 2D solar map raster was inputted back into ArcScene for full 3D view and compatibility. The original DEM was used together with the outputted solar maps in order to extrude a 3D solar radiation map/model from 2D ArcMap to 3D ArcScene output. The outputs were then overlayed onto the orthophoto with the correct georeferenced coordinates. This is illustrated in Figure 9 and Figure 10. 


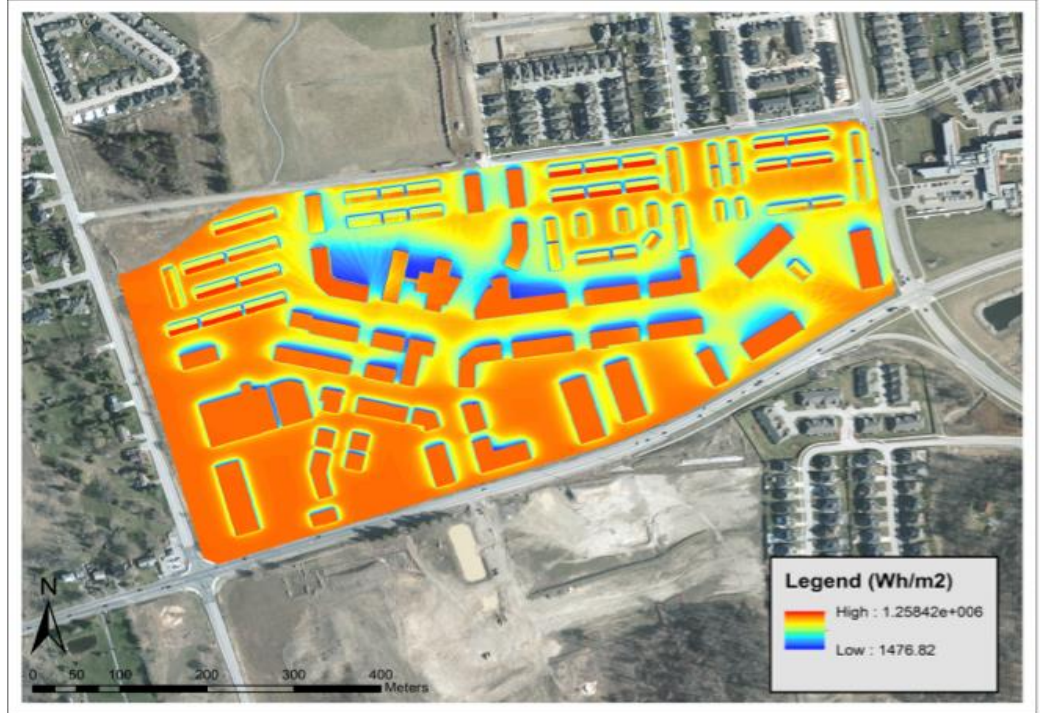

Figure 9. Example of an annual solar insolation map generated from the methodology for the original site plan.

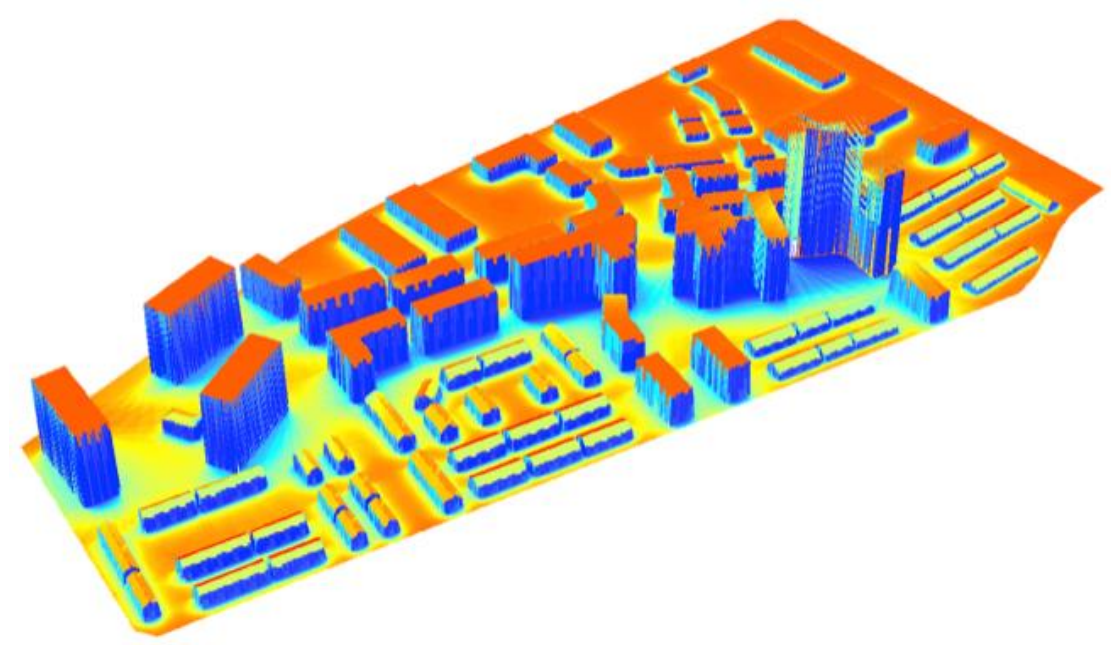

Figure 10. Extruded 3D solar insolation map in ArcScene based upon Figure 5 and original DEM elevation inputs.

It should be noted that this method is not capable of calculating insolation values on the building facades, however, when displayed in 3D in ArcScene, it does extrapolate the values from the original dataset onto the facades. Maps were generated for each daylight hour of the year and stored in a geodatabase for each model tested. 


\subsubsection{Extraction of Data}

A solar map was calculated for each sunlight hour over the course of a year totalling to the generation of approximately 2000 maps per case study area. The calculated solar maps contain detailed solar irradiation information over the entire case study area. The solar irradiation received on each building rooftop can be extracted through the identification of roof-top pixels using the building's footprints. A roof extraction procedure was used in order to isolate the pixelated areas on the rooftops that are of particular interest for solar PV installation. A traditional remote sensing approach was used for the identification and extraction of the objects of interest in the generated solar maps. This approach is called the digitization process, which consists of a manual task of using image analysis and digitizing tools to extract the desired information. The extracted data can be attributed and validated during this geospatial process (Blundell \& Opitz, 2006). The extracted rooftop pixels are then put into a raster statistics calculating software tool called Zonal Statistics (under Spatial Analyst) in order to extract the hourly solar irradiation values into tabular format from the 2000 maps. With the generated hourly solar irradiation values $\left(\mathrm{Wh} / \mathrm{m}^{2}\right)$, it is possible to determine the PV potential of the buildings desired by converting the amount of solar falling on a given area ( $\mathrm{Wh} / \mathrm{m}^{2} / \mathrm{year}$ ) into the energy that a standard PV system would produce. Multiplication by the system efficiency to take into account for the standard test illumination for PV modules, temperature effects, cables and inverter losses would then follow. Full extraction of the hourly solar irradiation values from the rooftop pixels was attempted, however, an efficient iterative function was needed in order to speed the extraction process on the hourly maps over an annual basis.

\subsection{Benchmarking of 3D Solar Simulation Software Tools}

This section of the thesis will be focused on the benchmarking of the 3D solar PV simulation software tools. In brief, this section will begin by discussing the software tool selection criteria in order to determine which software tools should be included in the benchmarking analysis. It will then be followed by the performance analysis of the software tools against measured data and the determination of the physical functions and capabilities of the software tools. 


\subsubsection{Software Tool Selection Criteria}

In order to determine what solar simulation software tools would be suitable for 3D solar PV community energy analysis, a list of criteria was made in order to determine whether the software tool should be included in the benchmarking study. This list of criteria was established through the literature review in order to determine what is important for the simulation of $3 \mathrm{D}$ solar PV energy modeling for multiple buildings. The requirements that the software needed were:

1) A software that has the ability to model in a $3 \mathrm{D}$ environment

2) A software that is able to draw more than one building

3) A software that has the ability to analyze the solar potential on vertical facades

4) Has the ability to import weather data

5) Has the ability to model hourly resolution timestep

6) Has the ability to generate PV electricity results

7) A software that works on a typical desktop computer in an average office setting

8) Available in the English language

9) Not obsolete or discontinued

10) Available for Academic usage

From the list of solar simulation software tools that were identified in the literature review in Section 2.7, the tools were assessed to determine whether the above ten requirements were satisfied. For the GIS based solar models identified in Section 2.7.1, the software tools were not able to directly calculate criteria number three and six for façade analysis and generation of PV electricity results and were hence eliminated from the list. From the software tools identified in Section 2.7.2, ESP-r was found to be obsolete; and PVLib and PVWatts were not available in a 3D modeling environment. Thus, eliminating these software tools from the analysis as well. Lastly, from Section 2.7.3, regarding commercial software simulation tools, the following tools were eliminated from the list due to the following reasons:

- Skelion - Was not able to generate hourly electricity simulation results

- $\quad$ RETScreen - No 3D analysis options available

- HOMER Energy - No 3D analysis options available 
- Ecotect Analysis - Obsolete and discontinued

- Aurora Solar - Not available for Academic usage

Based upon the elimination of these software tools, the remaining software tools that satisfied the mandatory requirements were found to be:

1) HelioScope

2) PVsyst

3) Archelios Pro

4) PVSOL Premium

5) EnergyPlus

6) System Advisor Model (SAM)

Hence, these six software tools were used for the benchmarking study in this thesis. The benchmarking of the 3D PV solar simulation software tools was broken down into two main sections: (1) the simulation performance analysis against a case study area, and (2) the physical functions and capabilities of the software tools.

\subsubsection{Performance Analysis Simulations}

A case study area was required for the performance analysis of the six software tools. This discussion will start by providing a description of the case study area, data acquisition, and analysis procedures performed in the 3D solar PV software tools.

\subsubsection{Case study area}

A case study area with an existing PV installation was needed for the assessment of the solar PV simulation software tools. A net-zero energy house located in Strathroy, near London Ontario, Canada, was selected for the case study area. This net-zero energy house was constructed in 2017 and has a rooftop PV array with a total capacity of $8.745 \mathrm{kWp}$. Figure 11 is an image of the case study residential home. 


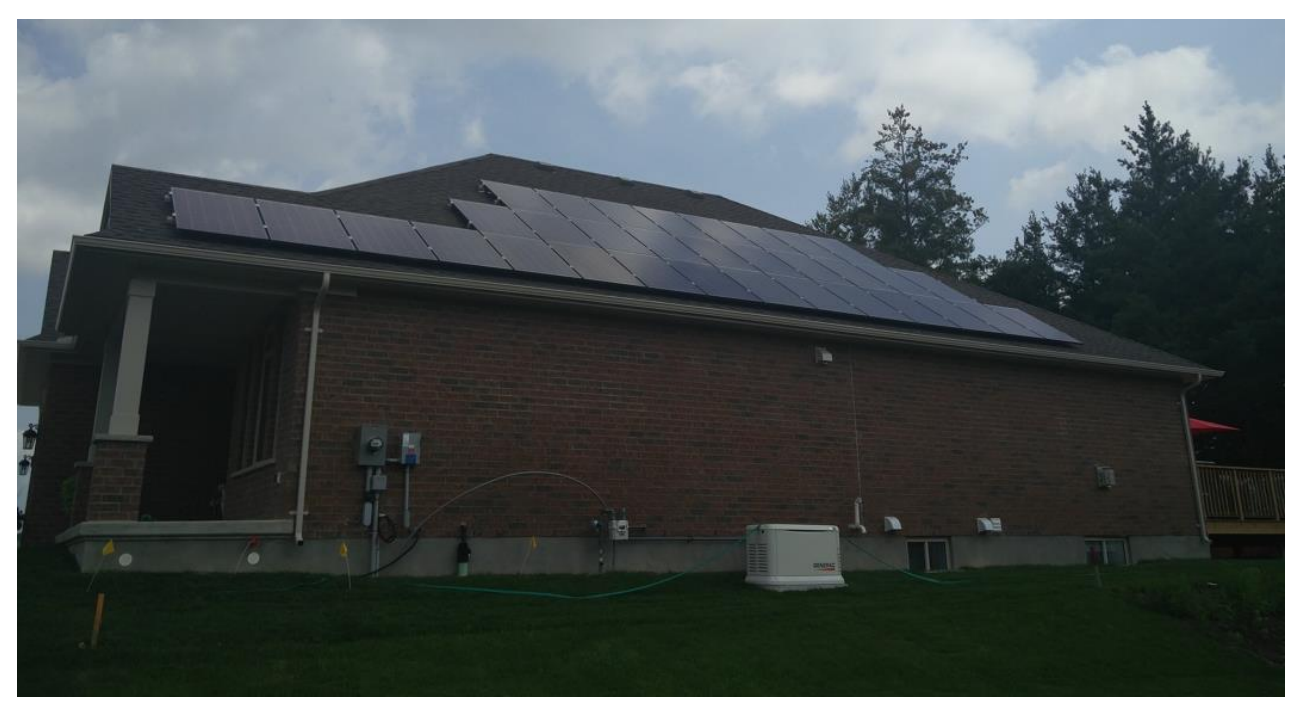

Figure 11. Photo of the case study residential home.

The solar PV module information, PV modeling specifications, inverter and optimizers information for the modeling of the case study area are summarized in Table 2 to Table 4 .

Table 2. Solar module information

\begin{tabular}{|c|c|}
\hline Manufacturer & Canadian Solar \\
\hline Model & CS6P 265 \\
\hline Module Type & Polycrystalline \\
\hline STC Power & $265 \mathrm{~W}$ \\
\hline Voc (Vmp) & $37.7 \mathrm{~V}(30.6 \mathrm{~V})$ \\
\hline Isc (Imp) & $9.23 \mathrm{~A}(8.66 \mathrm{~A})$ \\
\hline Temperature Coefficient & Pmax $=-0.41 \% /{ }^{\circ} \mathrm{C}$ \\
\hline Efficiency & $16.47 \%$ \\
\hline
\end{tabular}

Table 3. PV Modeling Specifications

\begin{tabular}{|c|c|}
\hline Total No. PV Modules & 33 \\
\hline Total DC Capacity & $8.745 \mathrm{~kW}$ \\
\hline No. PV Strings & 2 \\
\hline Branch Circuit Pmax & $4.505 \mathrm{~kW}$ \\
\hline
\end{tabular}




\begin{tabular}{|c|c|}
\hline Module Tilt & $33.69^{\circ}$ \\
\hline Roof Pitch & $8: 12$ \\
\hline Roof Azimuth & $180^{\circ}$ \\
\hline Module Surface Area & $53.1 \mathrm{~m}^{2}$ \\
\hline
\end{tabular}

Table 4. Inverters and Optimizers

\begin{tabular}{|c|c|}
\hline Inverter Manufacturer & SolarEdge \\
\hline Inverter Model & SolarEdge SE7600NA \\
\hline Total No. Inverters & 1 \\
\hline Inverter Capacity & $7.6 \mathrm{~kW}$ \\
\hline Optimizers (Y/N) & Y \\
\hline Optimizer Make Model & SolarEdge P300 \\
\hline Total No. Optimizers & 33 \\
\hline
\end{tabular}

The details from this installation setup was inputted into each solar simulation software tool.

\subsubsection{Data Collection}

Data for the hourly electricity generated by the PV system was collected for the case study area from January 1, 2018 to December 31, 2018. The collected data was then analyzed in order to make sure there were no errors or missing data. There were multiple occasions in which data was not collected or missing for several hours (missing timestamps or data). Those hours were removed from the sample dataset totalling to 216 hours being removed for the year. The relevant hours were removed from the simulation results as well.

Ideally, for a verification study, weather data collected on site for the total time period in consideration would provide the most accurate results. A weather sensor Rainwise PVmet-75 was installed on the south side of the house in which GHI $\left(\mathrm{W} / \mathrm{m}^{2}\right)$ and ambient air temperature $\left({ }^{\circ} \mathrm{C}\right)$ was measured. Due to calibration and sensor issues, data for January 2018 was not usable. The sensor was uninstalled on December $5^{\text {th }}, 2018$, and GHI values were hence unavailable for the rest of the month. However, GHI was not enough information to create a weather dataset for all of the software tools to perform analysis, hence, the standard TMY data from CWEC 2016 London (.epw) weather file format was chosen for simulation. 


\subsubsection{Simulation Procedures in the Software Tools}

Simulations were performed on the case study area in six software tools: (1) HelioScope, (2) PVsyst, (3) PVSOL, (4) Archelios, (5) EnergyPlus and (6) SAM. The general simulation procedure employed using these software tools is depicted in Figure 12.

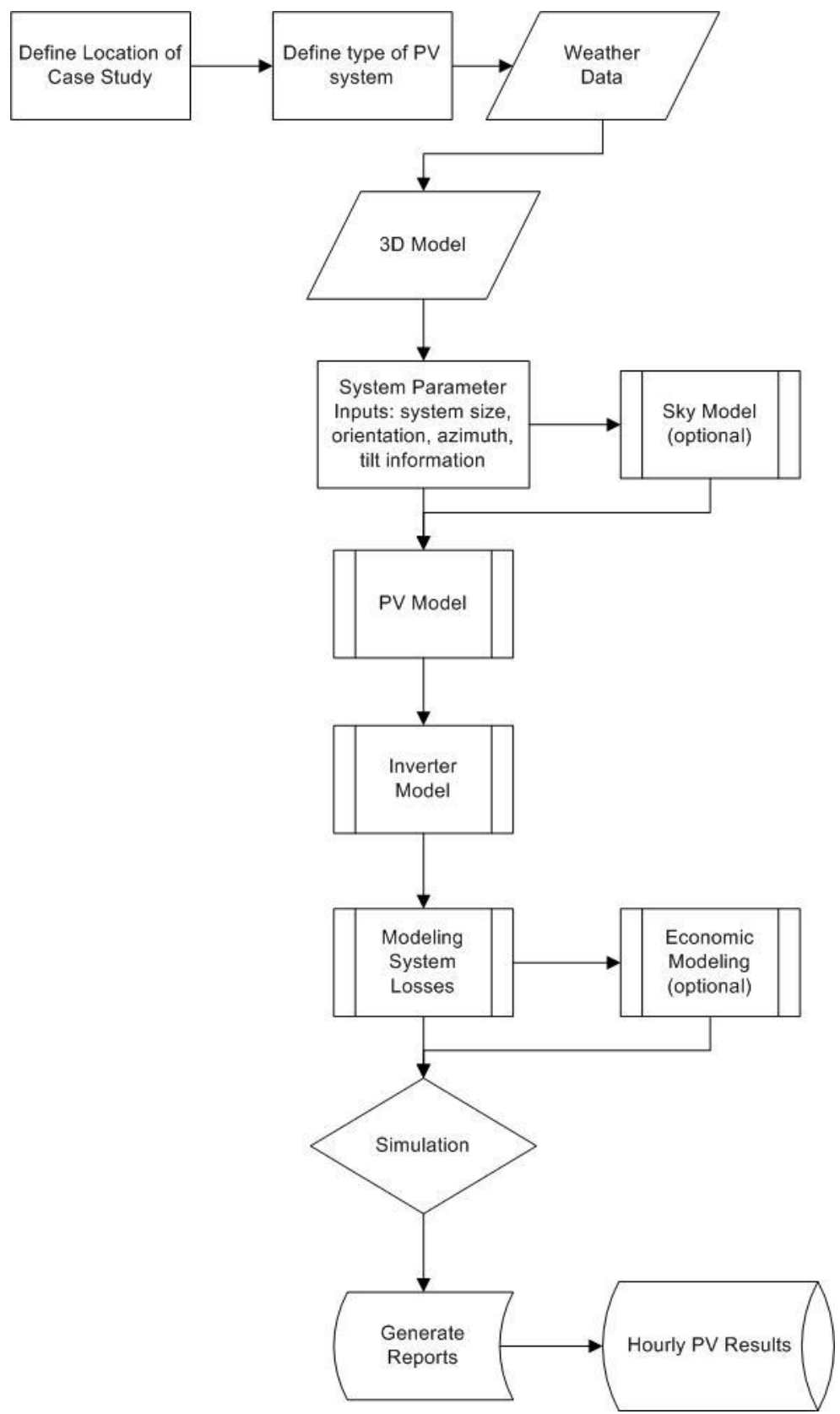

Figure 12. Overall simulation procedure workflow 
The software tools were modeled with the data specifications for the case study area as aforementioned. In order to maintain consistency in the modeling process, some decisions needed to be made regarding the modeling of certain functions in certain software tools, as all tools do not have the exact same functionality. Note that the scope of the comparison of the software tools will be focused only on the physical functions and capabilities and will not address the optional economic or life cycle assessment modeling aspects of the software tools.

In each of the PV modeling software tools, there were many different models and default values to choose from which assume the behaviour of the system at different points throughout the modeling process. Although many of the internal models may be similar or the same, the inputs accepted or required are different. There were also particular differences in how the software tools handled the modeling of system losses (for example some would model 5 losses, and others 10 different types of losses). Although there are many options to choose from, each software tool does have its recommended or default models and values to select from. Hence, in order to provide consistency between the modeling process using the software tools, the simulations of the case study area were performed using the default models and values, except for the case of the solar radiation models. The solar radiation transposition models were modeled when possible to investigate if they made a difference in the outputs for the software. The detailed simulation procedures performed will now be discussed for (1) HelioScope, (2) PVsyst, (3) PVSOL, (4) Archelios, (5) EnergyPlus and (6) SAM.

\section{HelioScope}

HelioScope is a commercial subscription web-based application and was launched on Google Chrome for simulation in this thesis. The simulation procedure workflow performed using HelioScope in this thesis can be divided into four main stages: (i) geolocation of the project area, (ii) creation of Design layout, (iii) creation of Condition set, and (iv) simulation.

(i) Geolocation of the project area

To begin a new simulation in HelioScope, the location of the case study area needed to be located on the online mapping interface, using its address or geographical coordinates. The 
default satellite imagery used in HelioScope is from Google Earth. After defining the location of the case study area, a Design and Condition set needed to be created for the simulation.

(ii) Creation of the Design layout

A Design layout in HelioScope contains the information for the geographic location of the solar PV, the detailed module layout, orientation, roof shape, and surrounding objects or obstructions for the simulation. This is where the mechanical and electrical layouts for the PV system were defined.

In the mechanical layout section, the first task was to locate the rooftop of the case study area in order to trace it out and extrude it from the building footprint on the map to create a 3D model. Note that it was not possible to import any existing 3D CAD models into HelioScope, and hence the 3D model had to be recreated from the satellite imagery within the software tool. This extruded model defined the field segment of the rooftop to be filled with the PV modules. The PV model for the case study area Canadian Solar CS6P-265P was selected from HelioScope's PV module database. The layout, tilt, azimuth, row spacing, setbacks and racking assumptions were then defined as indicated on Figure 13 .

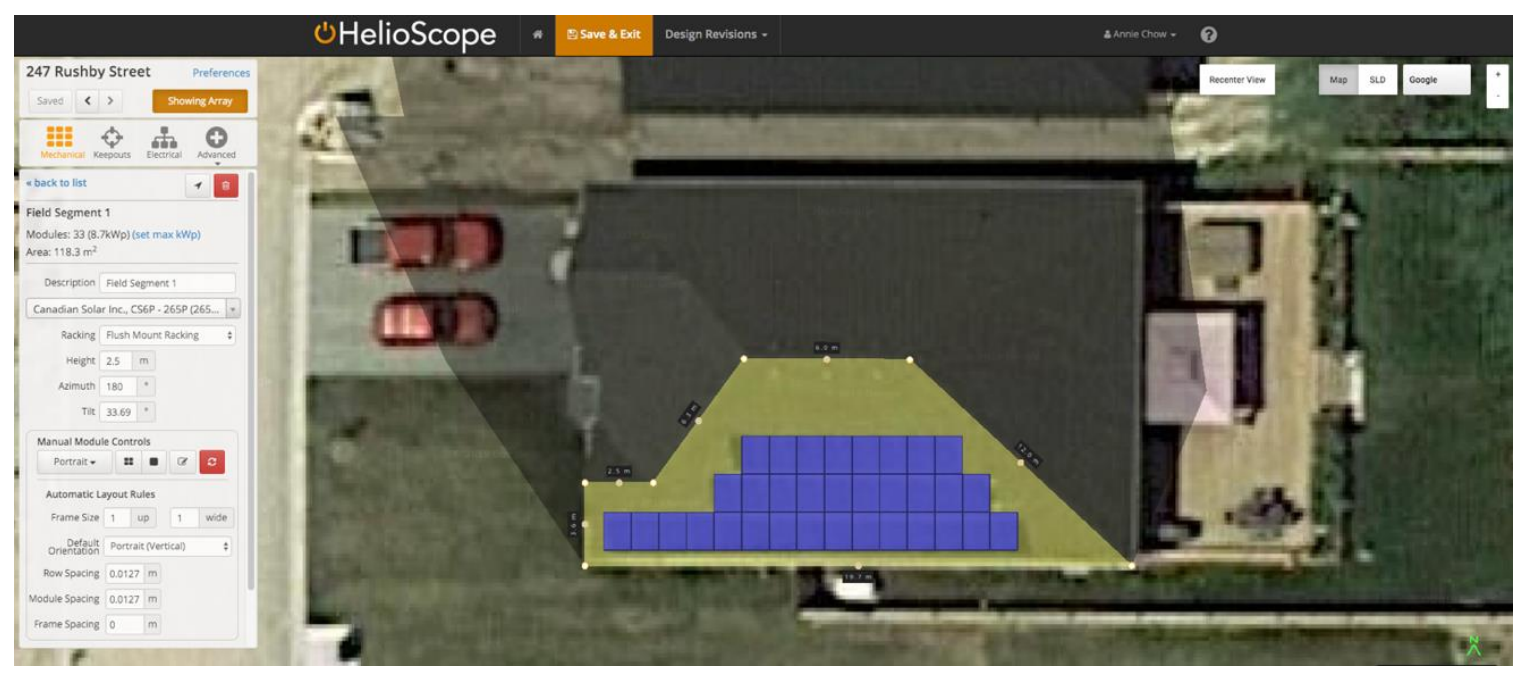

Figure 13. Mechanical layout design and selected parameters for case study area.

The next step was to define any 'keepouts' which are obstructions or areas to be excluded from the analysis that generates shade on your case study area. This accounts for chimneys, maintenance paths, HVAC units, and so forth. 
The surrounding buildings were modeled as keepouts in the case study area. The height of the surrounding buildings were estimated by using the shade based height estimation method which estimates the height of a building based upon the length of its shadow at a particular date and time. The date the Google Earth Pro image was taken for the case study area was on July 2 , 2018, and based upon that date, the time of day and shadows were matched to the height of each building, to get a rough estimate of their height. Figure 14 is an example of the case study area with surrounding buildings and a few trees (keepouts) modeled.

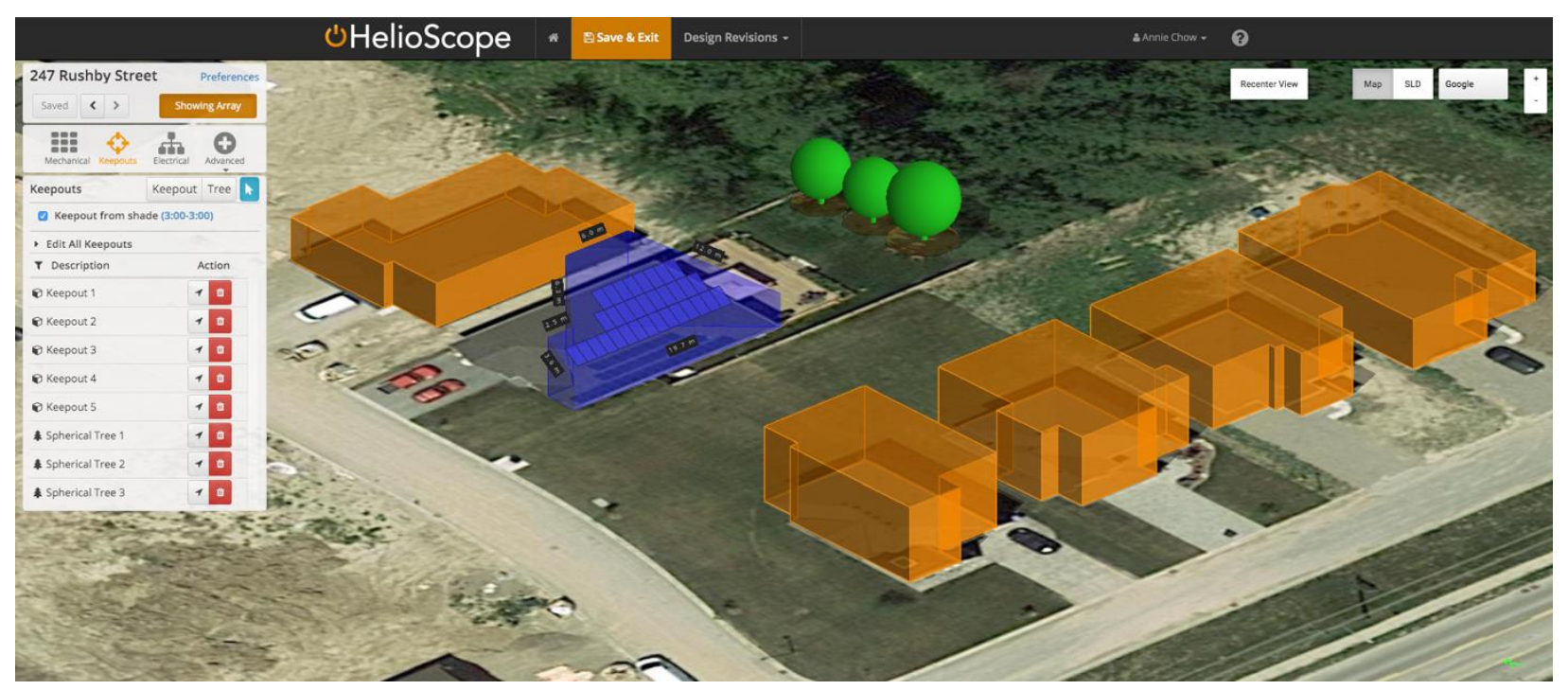

Figure 14. 3D view of case study area with neighbouring buildings and trees modeled as keepouts.

After the creation of the 3D modeling scene the next part of the simulation procedure was to define the electrical design. This part of the modeling process completes the mechanical layout by generating strings and connecting inverters to the wiring zones. The default inverter DC/AC ratio is 1.25 in HelioScope, that is it will automatically select the inverter count that achieves a DC/AC ratio as close to $125 \%$ without going over. The specifications for SolarEdge SE7600A inverter and P300 optimizer was added to the simulation from HelioScope's database and the system was configured to a 1.15 DC/AC ratio. The sizing of the string range was automatically calculated according to temperature from ASHRAE weather data. Figure 15 shows the electrical design parameters for the house. This step completes the Design Set for simulation. 


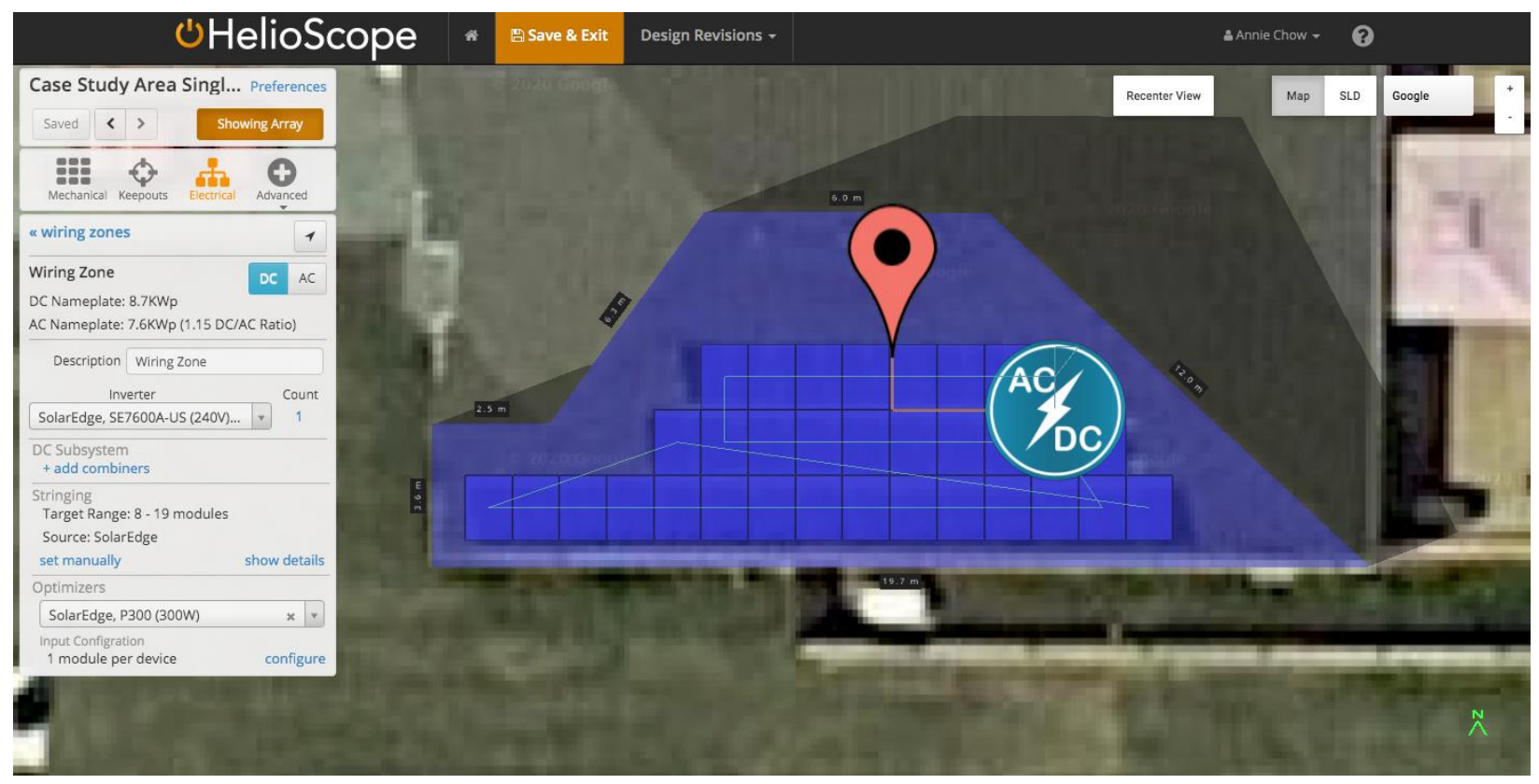

Figure 15. Electrical design layout of case study area with inverter and optimizers modeled.

(iii) Creation of the Condition sets

The next step was to create the Condition sets for the simulation. Condition sets are a mandatory component to the simulation procedure and is used in conjunction with a Design layout for any simulation. Condition sets describe the environmental conditions pertaining to the modeled solar array that contains a number of assumptions around the weather conditions, shading patterns, and soiling losses. For the weather data used in the Condition set, HelioScope has various meteorological sources and options available for the user to select from. Other major parameters such as solar radiation transposition model, horizon, and derate factors are located here as well.

Two Condition Sets were created in HelioScope for simulation, since it offers the choice between two transposition models, the Perez and Hay model. The default model is Perez. The CWEC London 2016 EPW weather file was uploaded to the weather dataset and the default parameters for soiling, cell temperature model, mismatch, and AC losses were kept. Figure 16 and Figure 17 depict the Condition Sets created for the simulation. 


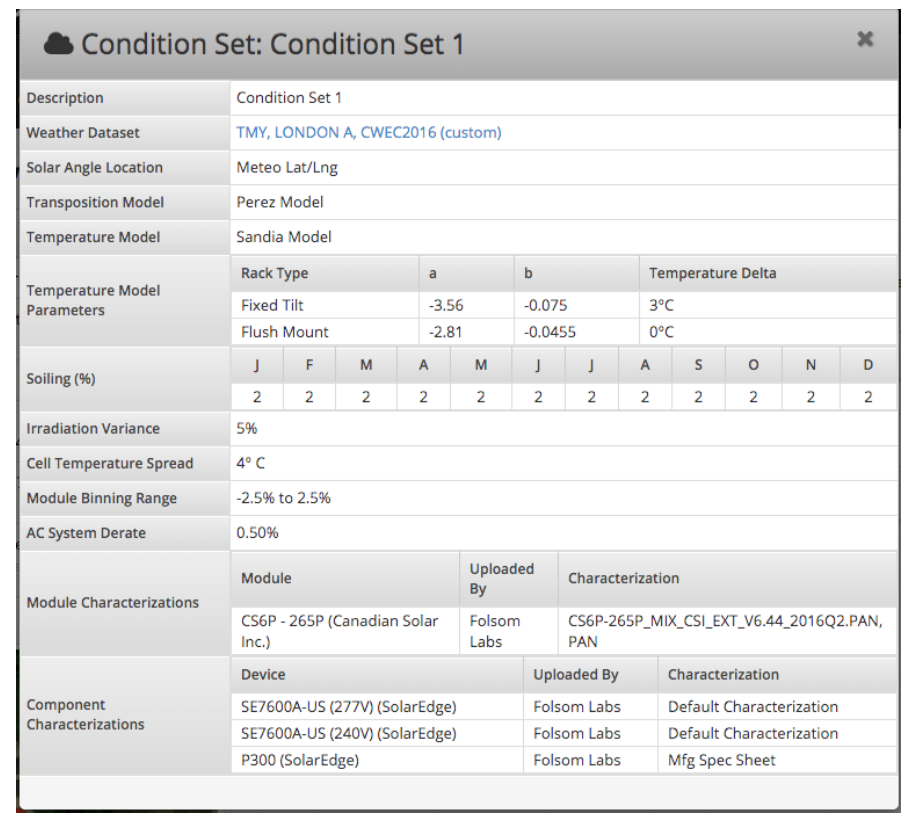

Figure 16. Condition Set 1 parameters - Perez transposition model

\begin{tabular}{|c|c|c|c|c|c|c|c|c|c|c|c|c|}
\hline \multicolumn{12}{|c|}{ Condition Set: Condition Set 2} & \multirow[t]{2}{*}{$x$} \\
\hline Description & \multicolumn{11}{|c|}{ Condition Set 2} & \\
\hline Weather Dataset & \multicolumn{12}{|c|}{ TMY, LONDON A, CWEC2016 (custom) } \\
\hline Solar Angle Location & \multicolumn{12}{|c|}{ Meteo Lat/Lng } \\
\hline Transposition Model & \multicolumn{12}{|c|}{ Hay Model } \\
\hline Temperature Model & \multicolumn{12}{|c|}{ Sandia Model } \\
\hline \multirow{5}{*}{$\begin{array}{l}\text { Temperature Model } \\
\text { Parameters }\end{array}$} & \multicolumn{3}{|c|}{ Rack Type } & \multicolumn{2}{|c|}{ a } & \multicolumn{2}{|c|}{ b } & \multicolumn{5}{|c|}{ Temperature Delta } \\
\hline & \multicolumn{3}{|c|}{ Fixed Tilt } & \multicolumn{2}{|c|}{-3.56} & \multicolumn{2}{|c|}{-0.075} & \multicolumn{5}{|c|}{$3^{\circ} \mathrm{C}$} \\
\hline & \multicolumn{3}{|c|}{ Flush Mount } & \multicolumn{2}{|c|}{-2.81} & \multicolumn{2}{|c|}{-0.0455} & \multicolumn{5}{|c|}{$0^{\circ} \mathrm{C}$} \\
\hline & \multicolumn{3}{|c|}{ East-West } & \multicolumn{2}{|c|}{-3.56} & \multicolumn{2}{|c|}{-0.075} & \multicolumn{5}{|c|}{$3^{\circ} \mathrm{C}$} \\
\hline & \multicolumn{3}{|c|}{ Carport } & \multicolumn{2}{|c|}{-3.56} & \multicolumn{2}{|c|}{-0.075} & \multicolumn{5}{|c|}{$3^{\circ} \mathrm{C}$} \\
\hline \multirow{2}{*}{ Soiling (\%) } & J & $\mathrm{F}$ & M & A & M & J & J & A & s & $\circ$ & $\mathrm{N}$ & D \\
\hline & 2 & 2 & 2 & 2 & 2 & 2 & 2 & 2 & 2 & 2 & 2 & 2 \\
\hline Irradiation Variance & \multicolumn{12}{|l|}{$5 \%$} \\
\hline Cell Temperature Spread & \multicolumn{12}{|l|}{$4^{\circ} \mathrm{C}$} \\
\hline Module Binning Range & $-2.5 \%$ & $2.5 \%$ & & & & & & & & & & \\
\hline AC System Derate & $0.50 \%$ & & & & & & & & & & & \\
\hline Module Characterizations & Modi & & & & $\begin{array}{l}\text { Uploaded } \\
\text { By }\end{array}$ & & Characte & izati & & & & \\
\hline & $\begin{array}{l}\text { CS6P } \\
\text { Inc.) }\end{array}$ & 265P & nadi & & $\begin{array}{l}\text { Folsom } \\
\text { Labs }\end{array}$ & & $\begin{array}{l}\text { CS6P-26 } \\
\text { PAN }\end{array}$ & P_M & $x_{-}$CSI_ & V6.4 & 2016Q2 & PAN, \\
\hline & Devic & & & & & Uplo & oaded By & & charac & zation & & \\
\hline Component & SE76 & A-US & 7V) & rEdg & & Fols & iom Labs & & Defaul & naract & ization & \\
\hline Characterizations & SE76 & A-US & ove ( & rEdg & & Fols & om Labs & & Defaul & naract & ization & \\
\hline & P300 & Solare & & & & & iom Labs & & $\mathrm{Mfg} \mathrm{Sp}$ & Sheet & & \\
\hline
\end{tabular}

Figure 17. Condition Set 2 parameters - Hay model

(iv) Simulation

A total of two hourly simulations were performed in HelioScope with the single rooftop Design layout (for consistency with other software tools analyzed) and the two Condition sets for the Perez and Hay model. Hourly PV electricity generation results were obtained from the 
simulations and simulation reports were generated by the software tool for the end user to show to a potential client (an example is depicted in Appendix B).

2. PVsyst

Simulations were performed for this thesis using PVsyst V6.78. A summary of the simulation procedure performed can be broken down into: (i) defining a new project, (ii) importation of the 3D model, (iii) system parameter setup, and (iv) simulations.

(i) Defining a new project

The case study area was simulated in PVsyst by first creating a new project design and defining the system type. The options for system type were grid-connected, stand-alone, pumping systems, and DC-grid connected systems. The system was set to grid-connected. The CWEC London 2016 epw weather file was then imported into the project which defined the geographical location and meteorological data required for the input parameters.

(ii) Importation of the 3D model

The 3D model of the case study area was imported into PVsyst using the "Near Shadings" function. Since PVsyst only accepts 3D Files of the types 3DS and DAE, the SketchUp file was converted to DAE format for compatibility. This allowed for the creation of the exact 3D geometry of the case study area in order to analyze the impacts of potential surrounding obstructions. Figure 18 presents a depiction of the imported case study area into the shading scene constructor in PVsyst. 


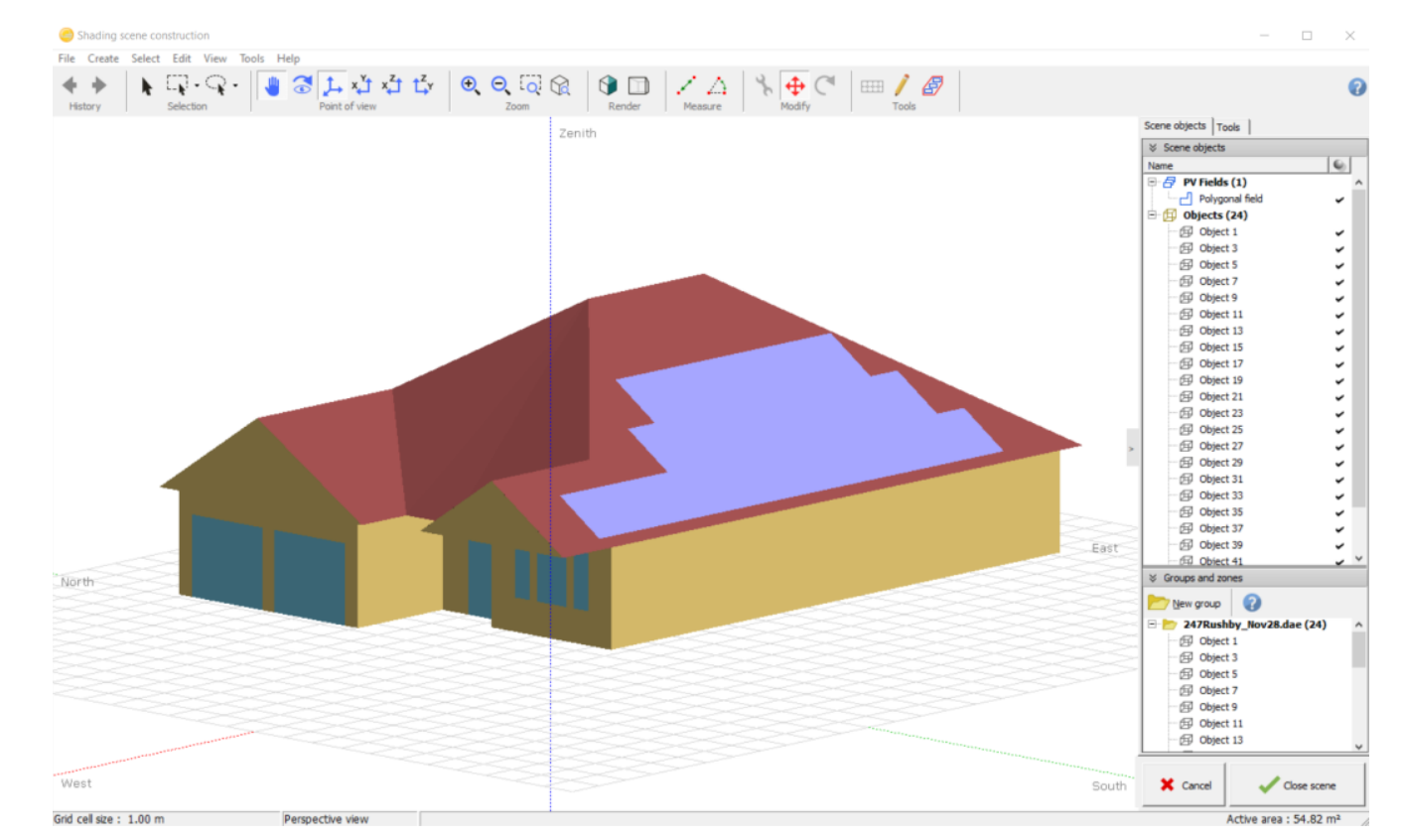

Figure 18. Imported 3D file of case study area into "near shadings" the 3D shading scene constructor for PVsyst.

(iii) System parameter setup

There were several parameters that need to be inputted in order for the software to run. The main input parameters that needed to be defined included: orientation, system, detailed losses, self-consumption, and storage. Note that self-consumption and storage options are not mandatory and were not necessary for this case study. For the orientation of the model, the plane of azimuth in PVsyst was set to $0^{\circ}$, as it is defined in PVsyst for the northern hemisphere as the angle between the south and collector plane, and the tilt of the plane was set to $34^{\circ}$.

PVsyst has a very large solar PV and inverter component database that is updated regularly. The solar PV model Canadian Solar CS6P-265P was selected from the database, as well as the SolarEdge SE7600A inverter and P300 optimizer. These components were defined in the main parameters input page. The 33 panels were connected in 2 strings in parallel, 1 string of 16 modules in series, and 1 string of 17 modules in series. The module layout tool was used to configure the string setup and the 3D drawing. Figure 19 shows the system configuration for the selected models. 


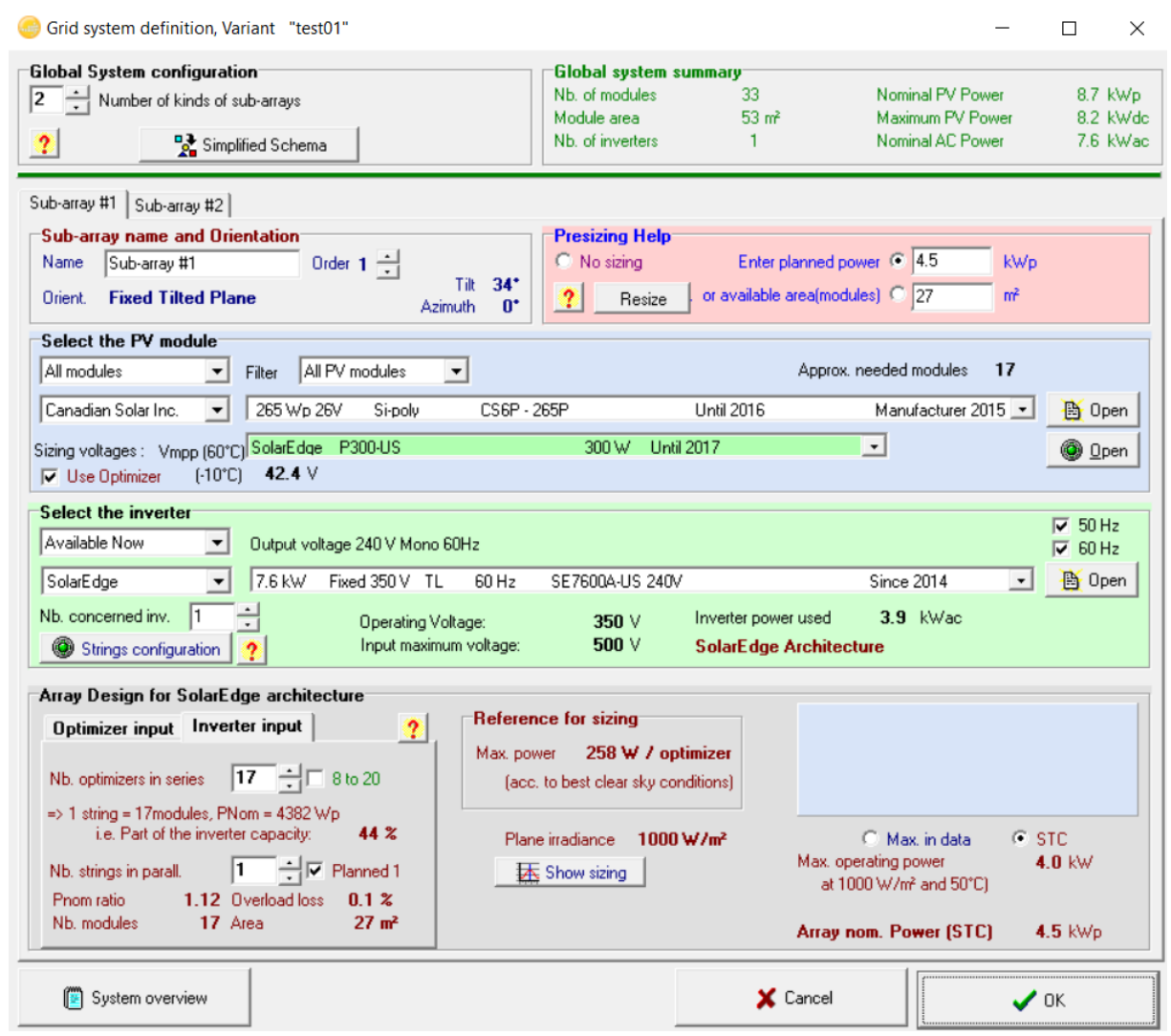

Figure 19. System Parameter setup for the case study area.

The default loss simulation parameters recommended by PVsyst for soiling, thermal, IAM, etc. were used for the simulation.

(iv) Simulations

For the final simulations, a total of two annual hourly simulations were performed in PVsyst, one for each of its transposition models, the Perez and Hay model.

3. $\mathrm{PV} * \mathrm{SOL}$

The version of PV*SOL Premium 2019 used for simulation in this thesis is R14. The major steps taken to perform the simulations in $\mathrm{PV} * \mathrm{SOL}$ premium consisted of the following main stages: (i) defining the system type, climate and grid, (ii) importation of the 3D model, (iii) PV and inverter setup, and (iv) simulations.

(i) Defining the system type, climate and grid 
When $\mathrm{PV} * \mathrm{SOL}$ was first launched, the type of system needed to be defined for the project. There are various types of system options available for modeling and they are: gridconnected PV system, grid-connected PV system with electrical appliances; grid-connected PV system with electrical appliances and battery system; grid-connected PV system with electrical appliances and electrical vehicles; grid-connected PV system with electrical appliances, electric vehicle and battery system; stand-alone PV system; and stand-alone PV system with backup generator. The system type for this case study was set to grid-connected PV system.

The next step was to select a weather data file for the location and climate of the case study area. The default weather data in PV*SOL uses data from Meteonorm, however, in order to maintain consistency with the rest of the software tools, the London CWEC 2016 epw weather file data was required to be used for simulation. The London weather epw file was not in a compatible format with $\mathrm{PV} * \mathrm{SOL}$ and needed to be converted into a suitable format. In order to convert the weather data, it had to be converted and imported in PV*SOL .dat weather format and it needed to contain hourly information for the following four parameters: ambient temperature $\left({ }^{\circ} \mathrm{C}\right)$, global radiation horizontal $\left(\mathrm{Wh} / \mathrm{m}^{2}\right)$, wind velocity $(\mathrm{m} / \mathrm{s})$ and relative humidity $(\%)$.

Data for those four parameters were extracted from the London CWEC 2016 epw file using the EnergyPlus Weather Statistics and Conversions software tool. The extracted data was then compiled into a text file and finally converted into *.dat file format for PV*SOL. The successfully converted weather file was then imported into PV*SOL.

(ii) Importation of the 3D model

The 3D CAD file of the case study area in Collada (dae) format was imported into the 3D editor of PV*SOL. Figure 20 depicts the case study area modeled in the 3D Editor of PV*SOL. 


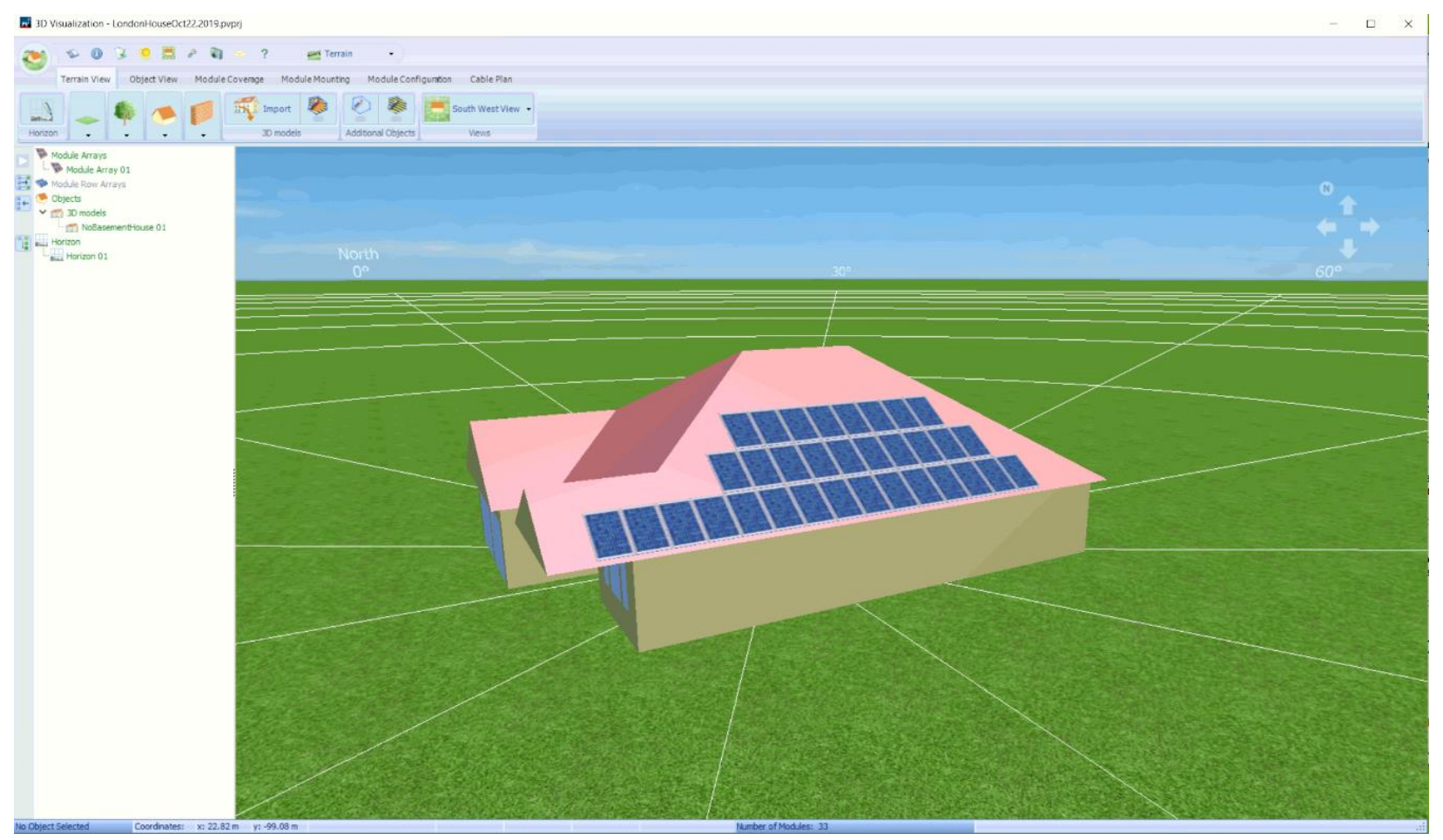

Figure 20. Case study house modeled in the $3 D$ Editor of $P V^{*} S O L$

(iii) PV and inverter setup

PV*SOL has a built in PV module and inverter database that contains a comprehensive list of manufacturers and PV module models and is updated regularly. The solar PV model Canadian Solar CS6P-265P was selected from the database, as well as the SolarEdge inverter SE7600A. The SolarEdge optimizer P300 US was not available in PV*SOL. There was the European P300 EU-APAC optimizer, however, it was not compatible with the SE7600A-US inverter due to strict rules dictated by the manufacturer for modeling, and PV*SOL would not run. The next closest optimizer was the P320 worldwide optimizer. The properties of the P320 optimizer and P300 EU-APAC were examined and compared (DC nominal input, max input current, max input voltage, max output current, max output voltage, characteristic curve and min MPP voltage), and they were found to be the exact same. Hence, the P320 optimizer was used for the simulation instead.

In order for the simulation to work in PV*SOL, the solar panels, inverters and optimizers must be configured and wired. The 33 modules were configured in two strings, comprised of 16 and 17 modules connected in series, respectively. The recommended cabling plan was used from 
$\mathrm{PV} * \mathrm{SOL}$ for configuration. A shade frequency calculation was performed in the module configuration area to determine if there are any obstructions on the solar panels. Figure 21 shows the module configuration that was performed in $\mathrm{PV} * \mathrm{SOL}$.

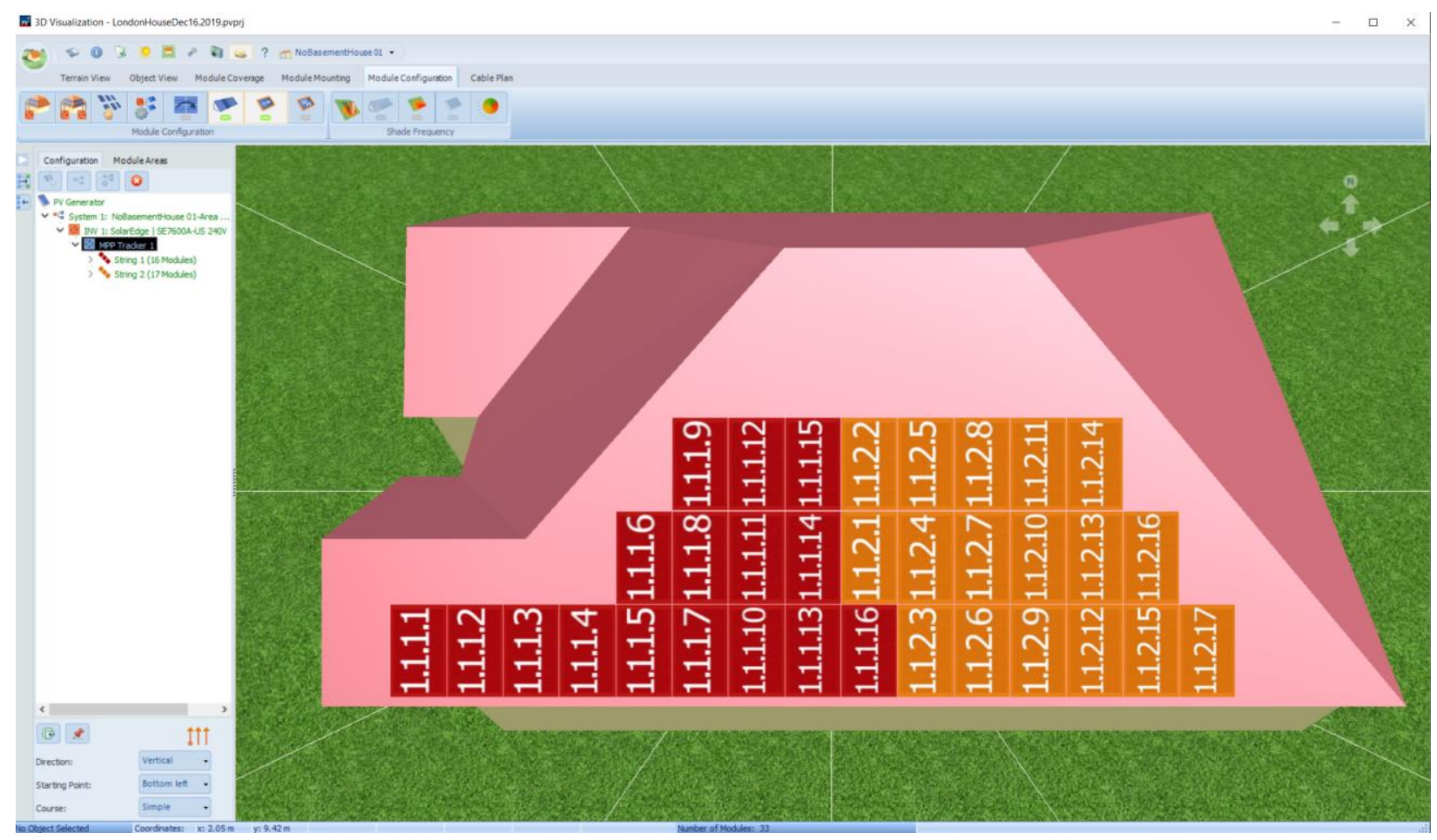

Figure 21. Module configuration plan in $P V^{*} S O L$.

(iv) Simulations

There were a few remaining simulation parameters that needed to be specified before simulation could begin. These parameters were related to the modeling of the losses of the system such as power losses due to voltage drop $(0.5 \%)$, loss of performance due to mismatch or reduced yield (2\%), ground reflection (20\%), and power losses due to soiling of the PV modules. These values were set to their default values as recommended by PV*SOL.

There was then the option to choose from five transposition models for simulation: (1) Hay \& Davies, (2) Liu \& Jordan, (3) Klucher, (4) Perez, and (5) Reindl. The Hay \& Davies is the default parameter set for simulation. A total of five simulations were performed in $\mathrm{PV} * \mathrm{SOL}$ using each of the five transposition models for analysis. 


\section{Archelios}

Simulation in Archelios Pro requires a subscription to online access as it is primarily a web-based application. The version of Archelios Pro used in this thesis is 2019R2.01. The modeling procedure that was performed in Archelio Pro can be summarized as follows: (i) 3D modeling in SketchUp, and (ii) Archelios Pro online simulation.

\section{(i) 3D modeling in SketchUp}

In order to create a 3D project in Archelios Pro, it required the usage of the Trimble SketchUp software. The Archelios Pro plugin was downloaded and installed into SketchUp. The 3D CAD model of the case study area was then imported into SketchUp and it's location was geolocated. With the Archelios Pro plugin toolbar, the PV module component database was accessed in order to select the type of solar panel for analysis. Canadian Solar CS6P-265P was selected from the module database and its design specifications were loaded. This allowed for the placement of the exact dimensions, azimuth and tilt of the solar panels onto the geometry of the rooftop as indicated on Figure 22.

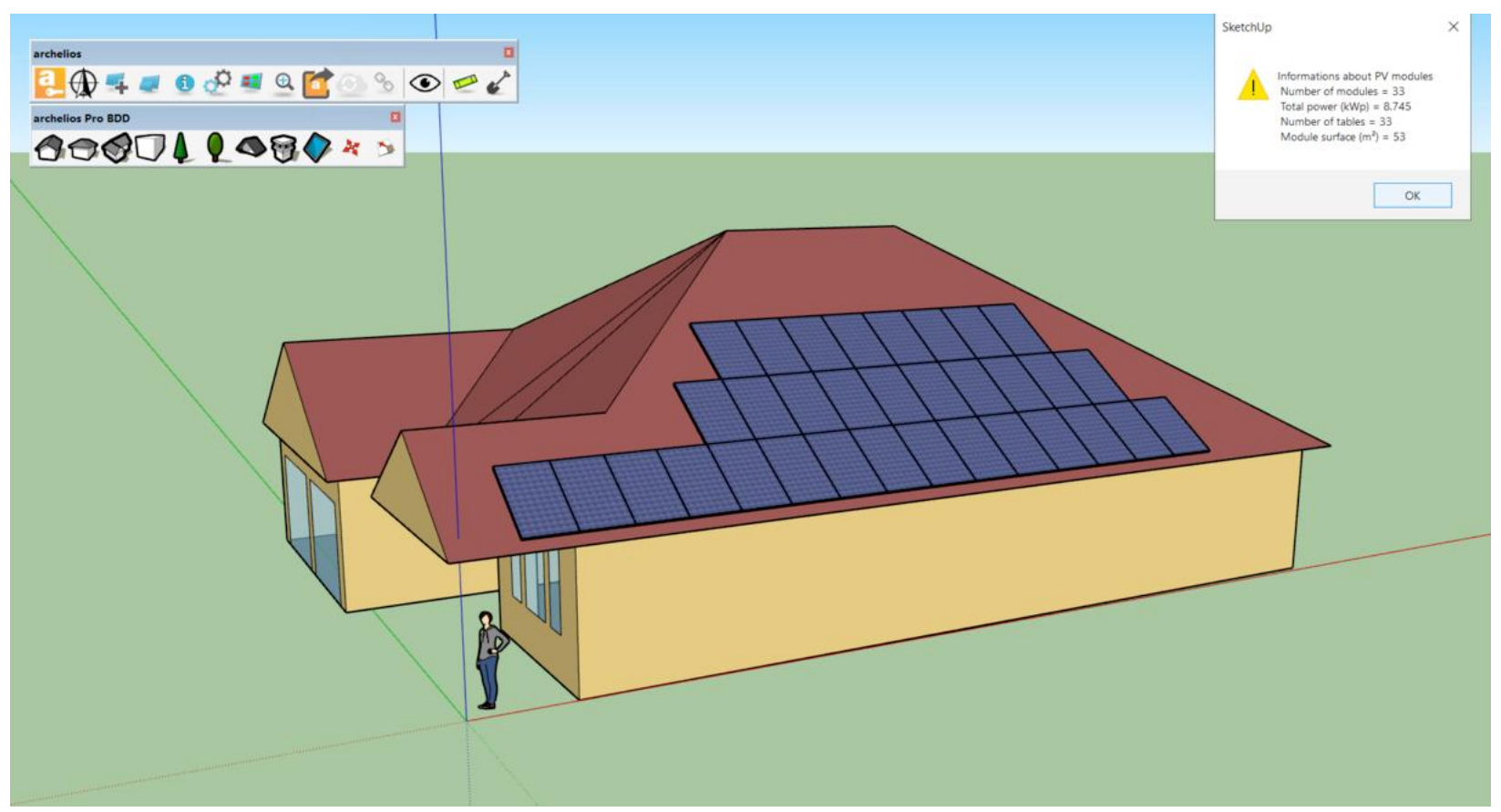

Figure 22. Case study area modeled in Trimble SketchUp with Archelios Pro plugin. 
The irradiation computation function in Archelios Pro was then used to quickly estimate the irradiation level for each PV module in the project design. This provided a 3D visualization of the amount of irradiation received on each PV module in color scale which is helpful to filter modules that are shaded or receive less than a specified minimum amount of irradiation per year. This takes into account the shading from surrounding objects and nearby obstructions. In the case of this study, there was no surrounding obstructions, as indicated by the green irradiation bars in Figure 23.

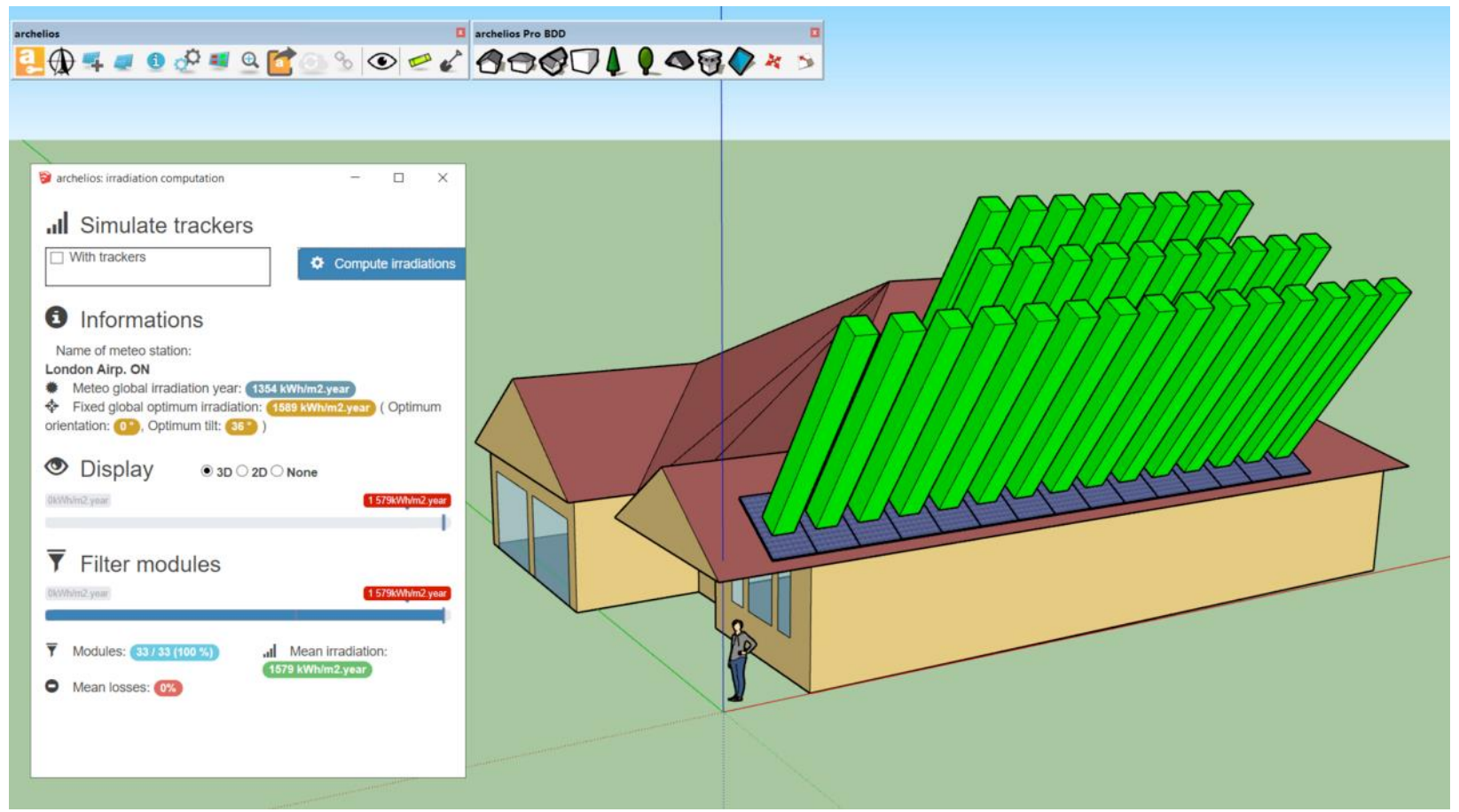

Figure 23. Irradiation computation in Archelios Pro for visualization of irradiation levels received per module.

After the completion of this step, the information from the 3D model was then exported onto the online Archelios Pro application for full simulation modeling capabilities.

\section{(ii) Archelios Pro online simulation}

Upon exportation of the SketchUp file to Archelios Pro online application, there are a few more simulation parameters and steps that need to be specified. By default, upon setting the geolocation of the project in SketchUp, Archelios will use the nearest reference station based upon Meteonorm data from the Archelios database. The London CWEC 2016 epw file was not 
in a compatible format for importation into Archelios and needed to be converted into a suitable format.

In order to import your own weather data, the user needs to provide the monthly mean data in Archelios meteo csv format: direct irradiance $\left(\mathrm{kWh} / \mathrm{m}^{2}\right)$, diffuse irradiance $\left(\mathrm{kWh} / \mathrm{m}^{2}\right)$, solar fraction $(\%)$, wind speed $(\mathrm{m} / \mathrm{s})$, air temperature $\left({ }^{\circ} \mathrm{C}\right)$, and link trouble (link turbity). This format is unlike other typical weather importation formats as it requires two additional variables solar fraction and link trouble. Due to the lack of data for the last two components, the London CWEC 2016 epw file was not able to be converted into a compatible format, and the default London Airport weather data was used from the Archelios database.

The next part of the simulation process required the configuration of the solar PV system. The SolarEdge SE7600A inverter and P300 optimizer was selected from the Archelios Pro database, however, this combination with 2 asymmetrical strings was not recognized as a valid configuration in Archelios Pro and the simulation would not compile. Other combinations of inverters and optimizers were recommended by the software tool that were compatible instead. Since Archelios is a proprietary software tool and there was no way to modify this issue, and it is not very flexible in its modeling processes. Despite various efforts, there was no way to configure the exact case study installation into Archelios Pro, and hence the simulation for the case study area was not possible.

\section{EnergyPlus}

In order to model and perform analysis in 3D using EnergyPlus, third party software was used, since EnergyPlus by default does not have a graphical user interface and is a command line interface simulation engine. The simulation for the case study area was performed using DesignBuilder V.6.1.3.008 and EnergyPlus V.8.9 in this thesis.

DesignBuilder provides a user-friendly modeling environment that is integrated with EnergyPlus and allows you to define your building and materials in a $3 \mathrm{D}$ environment. It allows for the easy exchange of data in a highly visually appealing interface and uses the EnergyPlus simulation engine to perform analysis. DesignBuilder was specifically developed around EnergyPlus which allows for most modeling functions such as databases of building materials, constructions, window panes, fabric and glazing data, etc. to be transferred. DesignBuilder can be used to generate the IDF files that EnergyPlus uses, and the IDF can be further customized if 
desired within EnergyPlus IDF Editor or programming for functions that are not built into DesignBuilder. The overall process that was undertaken to perform a simulation in DesignBuilder can be summarized as follows: (i) 3D modeling and weather data, (ii) PV model, (iii) inverter model, and (iv) simulations.

(i) 3D modeling and weather data

In order to perform a simulation, the "site:location" input objects need to be specified. The major parameters include: latitude, longitude, elevation, and time zone. The CWEC 2016 London epw file was loaded to the simulation. The next step was to define the case study area. The 3D CAD drawing of the case study area was not directly importable into DesignBuilder. The building had to be redrawn within the DesignBuilder environment. The rooftop solar PV panels were added using the "solar collectors" function to define the active PV area. The construction of the solar collectors can be defined in two ways: (1) solar hot water, and (2) photovoltaic. The "photovoltaic" selection was used. Note that it is not necessary to draw each and every PV module individually. One can draw an entire PV array using a representative total active surface area for the PV modules. Figure 24 depicts the completed 3D model of the case study area in DesignBuilder.

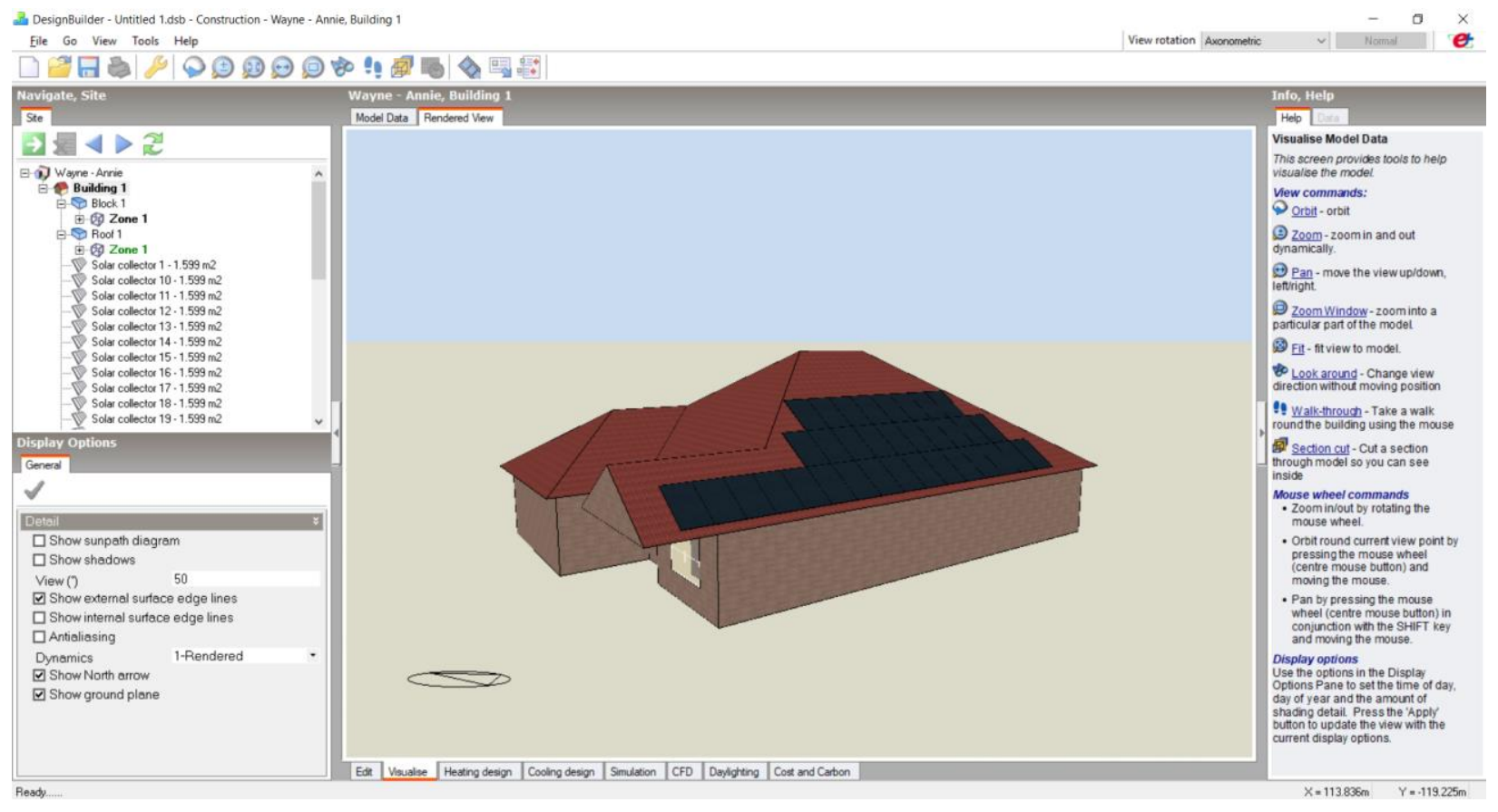

Figure 24. 3D model of case study area created in DesignBuilder. 
(ii) PV model

Once the 3D model was finalized with the weather data, parameters for the simulation needed to be defined. There are two PV modeling options available in DesignBuilder and they are: (1) Simple PV, and (2) Equivalent One-Diode model. Simulations using both these models were performed for the case study area. Note that in EnergyPlus there is a third PV model that was not built into DesignBuilder and that is the Sandia model. However, since the Sandia model uses specific performance input data needed for specific make and model types of PV panels using the empirical coefficients from Sandia, this model was not usable for the case study area.

The Simple PV model component is better suited for projects in the design phase, in which the exact PV specifications are unknown. It uses the efficiency of the PV panels to convert incident solar radiation to produce electricity estimates, and it does not require arrays of modules to be defined. The parameters used for the Simple PV model is depicted in Figure 25.

The Equivalent One-Diode model models the performance characteristics of the PV modules using the equivalent one-diode circuit. In order to use this model, specific testing information about the solar panel is required such as: shunt circuit current, open circuit voltage, voltage at maximum power, temperature coefficients for short and open circuit voltage, number if cells in series per module, cell temperature at NOCT conditions, and module area. A performance model was created based upon the solar PV manufacturer data sheet (Canadian Solar CS6P-265W) for the case study area. Usage of the equivalent one-diode model required that the number of modules in series, and series in parallel to be defined. Two series in parallel were modeled, with 16 modules in one, and 17 modules in the other string. Figure 26 shows the performance model parameters created for the simulation. 


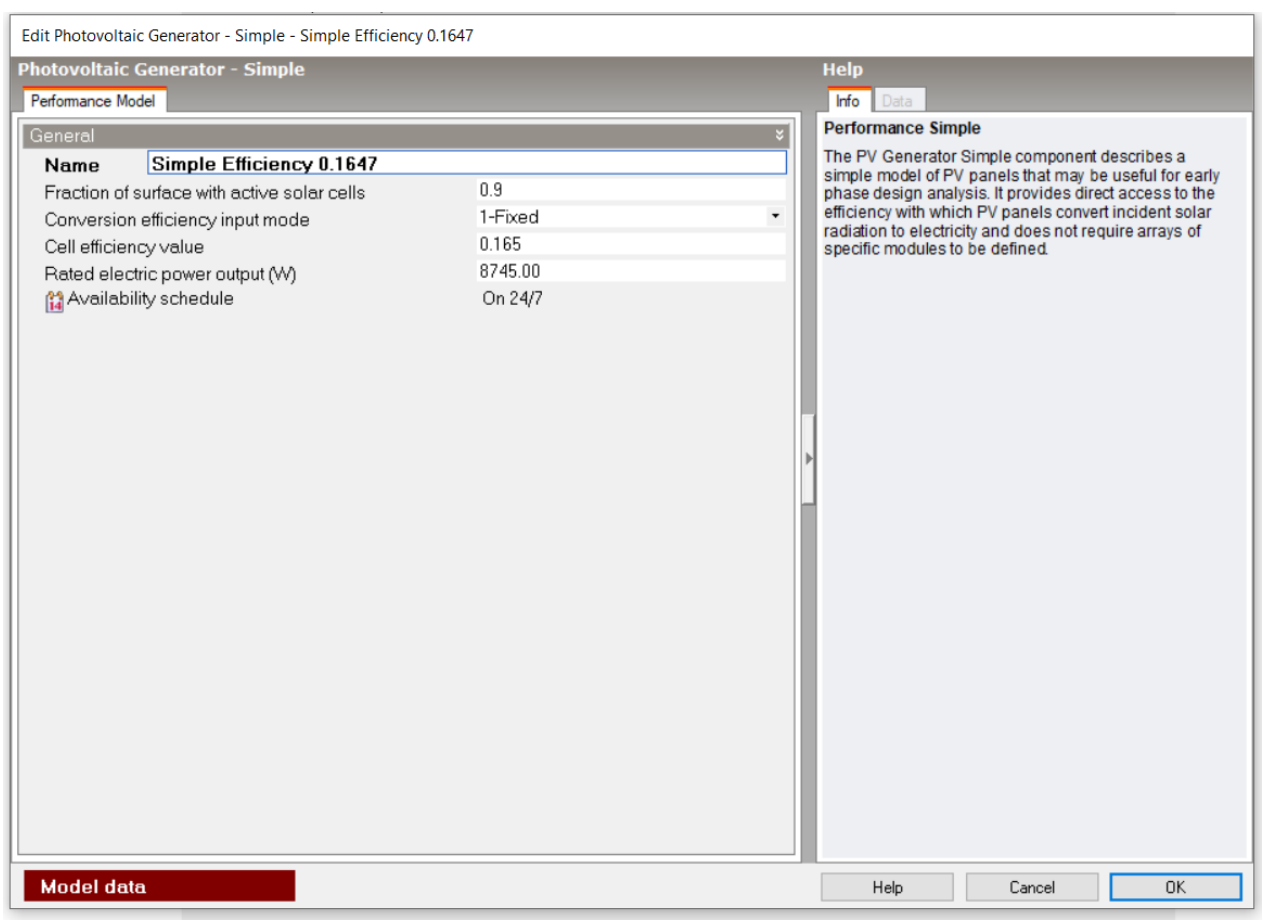

Figure 25. Simple PV model parameters used for simulation in DesignBuilder.

\begin{tabular}{|c|c|c|}
\hline \multirow{2}{*}{\multicolumn{2}{|c|}{$\begin{array}{l}\text { Photovoltaic Generator - One-Diode } \\
\text { Performance Model }\end{array}$}} & \multirow{2}{*}{$\begin{array}{l}\text { Help } \\
\text { Info Data }\end{array}$} \\
\hline & & \\
\hline \multicolumn{2}{|l|}{ General } & \multirow{26}{*}{$\begin{array}{l}\text { Performance One-Diode } \\
\text { Performance One-Diode component is used to } \\
\text { describe the performance characteristics of } \\
\text { Photovoltaic (PV) modules to be modelled using an } \\
\text { equivalent one-diode circuit. This model is also known } \\
\text { at the 4- or 5-parameter TRNSYS model for } \\
\text { photovoltaics. }\end{array}$} \\
\hline Name CS6P 265P-Annie & & \\
\hline Cell type & 1-Crystalline Silicon & \\
\hline Cells in series & 60 & \\
\hline Active area $(\mathrm{m} 2)$ & 1.46 & \\
\hline Transmittance absorptance product & 0.9020 & \\
\hline Semiconductor bandgap (eV) & 1.12 & \\
\hline Shunt resistance (ohms) & 1000000.00 & \\
\hline Reference temperature $\left({ }^{\circ} \mathrm{C}\right)$ & 25.00 & \\
\hline Reference insolation (W/m2) & 1000.00 & \\
\hline Module heat loss coefficient $(W / m 2-K)$ & 30.00 & \\
\hline Total heat capacity $(\mathrm{J} / \mathrm{m} 2-\mathrm{K})$ & 50000.00 & \\
\hline Rated electric power output per module (W) & 265.00 & \\
\hline [ia Availability schedule & PV panel efficiency: Always 0.1647 & \\
\hline \multicolumn{2}{|l|}{ Current } & \\
\hline Short circuit current (A) & 9.23 & \\
\hline Module current at max power (A) & 8.66 & \\
\hline Temperature coefficient of short circuit current (A/K) & 0.00489 & \\
\hline \multicolumn{2}{|l|}{ Voltage } & \\
\hline Open circuit voltage $(M)$ & 37.7 & \\
\hline Module voltage at max power $(M)$ & 30.6 & \\
\hline Temperature coefficient of open circuit voltage ( $/ K)$ & -0.117 & \\
\hline \multicolumn{2}{|c|}{ Nominal Operating Cell Temperature } & \\
\hline NOCT ambient temperature $\left({ }^{\circ} \mathrm{C}\right)$ & 20.00 & \\
\hline NOCT cell temperature $\left({ }^{\circ} \mathrm{C}\right)$ & 45.00 & \\
\hline NOCT insolation (W/m2) & 800 & \\
\hline Model data & & Cancel \\
\hline
\end{tabular}

Figure 26. Equivalent one-diode model parameters for simulation of Canadian Solar CS6P-265P in DesignBuilder.

\section{(iii) Inverter modeling}

In the next step of the simulation procedure, the behaviour of the inverter needed to be specified in order for the model to simulate. For on-site electricity generation, EnergyPlus 
requires the usage of a component called "electric load centres". An electric load centre is responsible for the dispatching of generators according to the operation schedules defined and tracks the amount of electricity. The electric load centre defines how the generation system behaves and in the case for PV electricity generation, it helps to model the inverter and DC to AC output. An electric load centre was created, and the efficiency of the inverter was defined for the case study area as indicated in Figure 27.

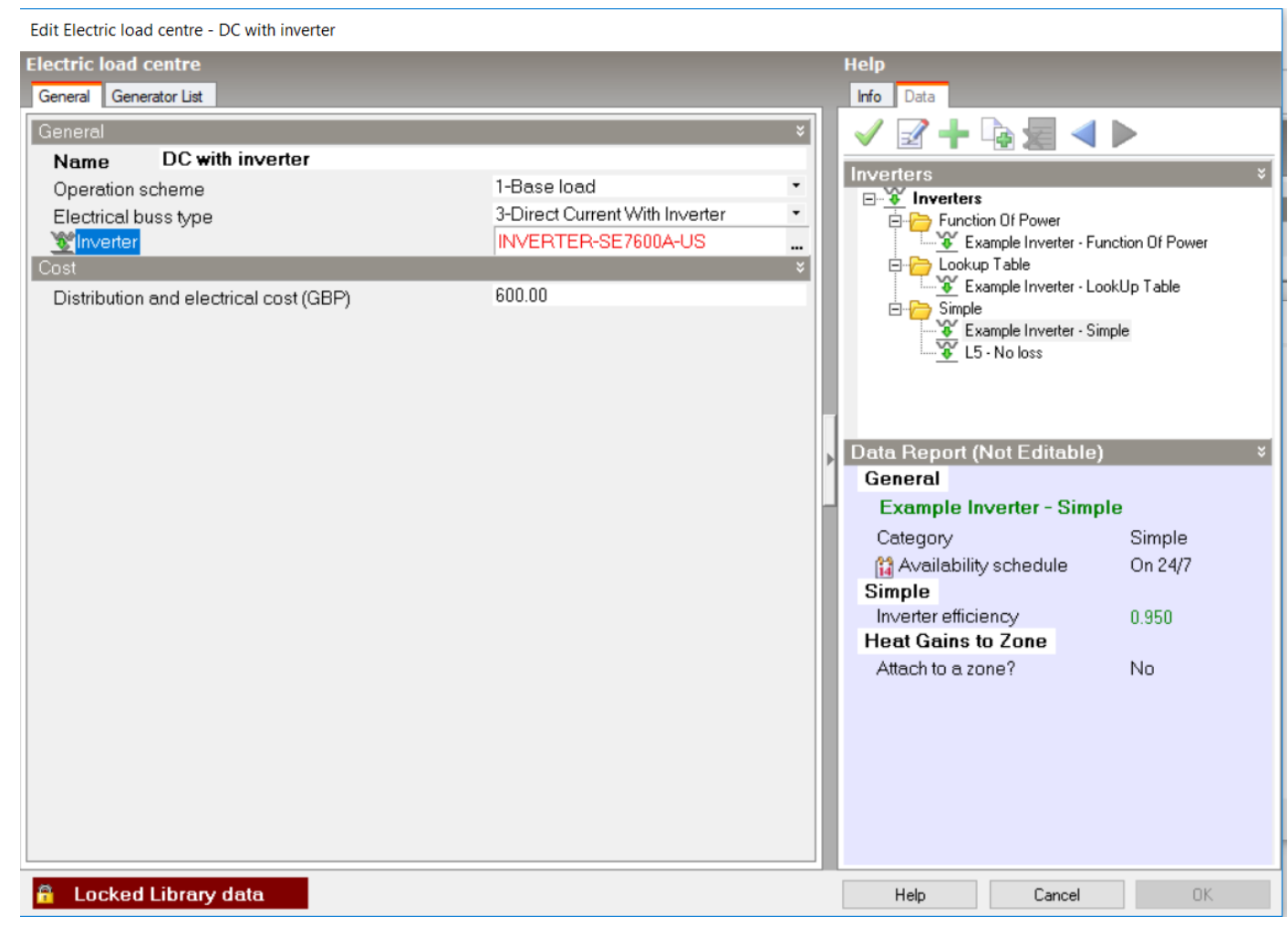

Figure 27. Electric load centre created for simulation.

Once the behaviour of the electric load centre was finalized, all the necessary components for simulation were defined.

(iv) Simulations

The hourly PV electricity potential was simulated for both the Simple PV model and Equivalent One-diode model options were simulated in EnergyPlus. There was no option available for changing the transposition sky radiation model in EnergyPlus (it only uses the Perez model), and hence both these scenarios were analyzed for comparison instead. 


\section{SAM}

The version of SAM used in this thesis is SAM 2018.11.11 Revision 4 SSC209. A simulation project in SAM is comprised of two main components, a performance model for the power system and a financial model for the project's financial structure. In order to begin a simulation, the performance model type, and financial model type must be first selected. There are many renewable energy system performance options available in SAM and three different types of solar PV models. The three solar performance models in SAM to select from are:

(1) The Photovoltaic (detailed) model - This model is used for the simulation of large- and small-scale PV systems when the information about the type of PV module and inverter in the system and specifications of the system's design are known. This requires detailed knowledge of the system. It calculates a grid-connected PV system's electrical output using separate module and inverter models. The user can input their own module and inverter specifications from the manufacturer data sheet or choose a module from the database.

(2) Photovoltaic (PVWatts) - This model uses NREL PVWatts model and is suitable for performing a general analysis for large- and small-scale PV systems when little information is known about the case study area. In order to use this model, the minimum inputs are just the location, system nameplate size, orientation, mounting type and tilt. This provides a quick estimation of the output when further details are not known yet for the exact specifications for PV module info etc. Using the PVWatts simulation model through SAM, enables all of SAM's financial modeling potential, something that is not possible to do directly on the PVWatts website itself. Also, the user will be able to generate shading reports also, whereas on the PVWatts website that is not available.

(3) High Concentrating Photovoltaic - This model is used for simulating large and small concentrating PV systems with high concentration PV modules. In order to use this model, it requires additional information about the design of the concentrator and efficiency of the cell at different irradiance levels. 
In this thesis, SAM'S Photovoltaic (detailed) performance model was selected for analysis with no financial model. The PVWatts model was not employed as that calculation is based upon the NREL PVWatts mapping website calculator. The workflow process for the simulation procedure can be summarized into these main stages: (i) location and resource, (ii) module, (iii) inverter, (iv) system design, (v) shading and layout, (vi) losses, and (vii) simulations.

\section{(i) Location}

The first step to performing the simulation was to input the location and solar resource information. SAM has a Solar Resource Library that you can download and add weather data into. SAM uses a CSV comma-separated text format for solar resource data in order to perform its solar performance model calculations. The CSV supports hourly and sub-hourly data with up to a one-minute resolution. The CWEC London $2016 \mathrm{epw}$ weather data file needed to be converted into a compatible format that SAM could read. One of the reasons is that the file contained extra information that was not necessary for the simulation to be performed. The Solar Resource File Converter Macro was used to convert the EPW file into a SAM CSV file format. The successfully converted weather file was then imported into the location for the simulation.

The albedo, sky diffuse model, and irradiance data inputs are also defined in this section of the simulation procedure. There are three options for sky diffuse model in SAM and they are: (1) Isotropic, (2) HDKR, and (3) Perez (default) sky diffuse models. Weather file irradiance data can be calculated in SAM in various combinations and they are: DNI and DHI, DNI and GHI, GHI and DHI, POA from reference cell, and POA from pyranometer.

For irradiance data inputs, SAM is recommended to be simulated using the DNI and DHI default option and is usually the best for most analyses. This was the option that was selected for the final simulations, since the weather data was based upon high quality CWEC data. SAM reads the DNI and DHI data from the weather file, and then calculates the incident irradiance (POA) using the DNI and DHI data from the weather file without performing any additional calculations.

\section{(ii) Module}

This stage is where the type of PV model is selected for simulation, and the module model is chosen. A PV module can be selected from the SAM database, or user inputted if the 
module is not available. In SAM, the module model estimates the DC output of an individual module every step of the way. The PV array output is dependent upon the system parameters of the design (such as the orientation, azimuth, tracking, shading and so forth). Note that an array can be further subdivided into a maximum of four subarrays. There were a few options to select from for module modeling at this step: CEC single-diode model, simple efficiency, Sandia and IEC61853 single-diode model.

The default module model is the single-diode model -CEC performance model based upon the PV module database. This is the most popular choice due to the large available database. The simple PV efficiency model was not used in this thesis as it is the least accurate out of the PV models for predicting the performance of modules (Blair et al., 2013). Data for the Sandia and IEC model were not available as well. Hence, the Canadian Solar CS6P-265P model was selected from the database and run with the CEC single-diode model based upon its module database.

(iii) Inverter

After the selection of the PV modules, the next step in the simulation procedure would be to select the inverter performance model and inverter type from the database. The user can also choose to enter their own inverter parameters from manufacturer's data if desired. The default inverter CEC database method was chosen for simulation since the information for the SolarEdge SE7600A inverter was already included in the database and was the only viable option. Note that there are no options for selecting optimizers available. The calculation for the optimizer in SAM was adjusted for in the losses section of the simulation procedure.

(iv) System Design

The System Design Page in SAM is where the detailed information for the setup of the PV array is inputted. This is where the specific PV sizing and configuration for the system is performed, as well as the parameters such as azimuth, tilt, and orientation are inputted. The PV system sizing was configured in SAM according to the case study parameters, and the PV array was modelled as 2 strings connected in parallel, one string of 16 and one string of 17 modules connected in series. 
(v) Shading and layout

This is where the 3D shade calculator can be used for 3D modeling to discover the shading losses. SAM's 3D shade calculator stores and reads 3D scene data from files in the .s3d format only. It cannot directly read files in other formats and it cannot import 3D CAD drawings. Hence, the case study area had to be redrawn in SAM. SAM's 3D modeling interface does not have much flexibility like most 3D CAD based software tools and has limited drawing abilities. You can only draw four types of predefined objects in 3D: a box, cylinder, roof and tree. The PV array is drawn on as an 'active surface'. The aim of the $3 \mathrm{D}$ shade calculator is to help users perform basic analysis of shading impacts on PV power production for a particular scene. The drawing is meant to be a representative model of the case study area and not a precise drawing. Figure 28 depicts the case study area drawn in SAM's 3D shading scene editor. This 3D shade calculator will calculate a month by month-by-hour shading loss table of beam irradiance shade loss percentages and a sky diffuse loss percentage based upon the model. An optional satellite imagery was obtained from Google maps was used as an underlay to the scene for visualization and proportion purposes as shown in Figure 29.

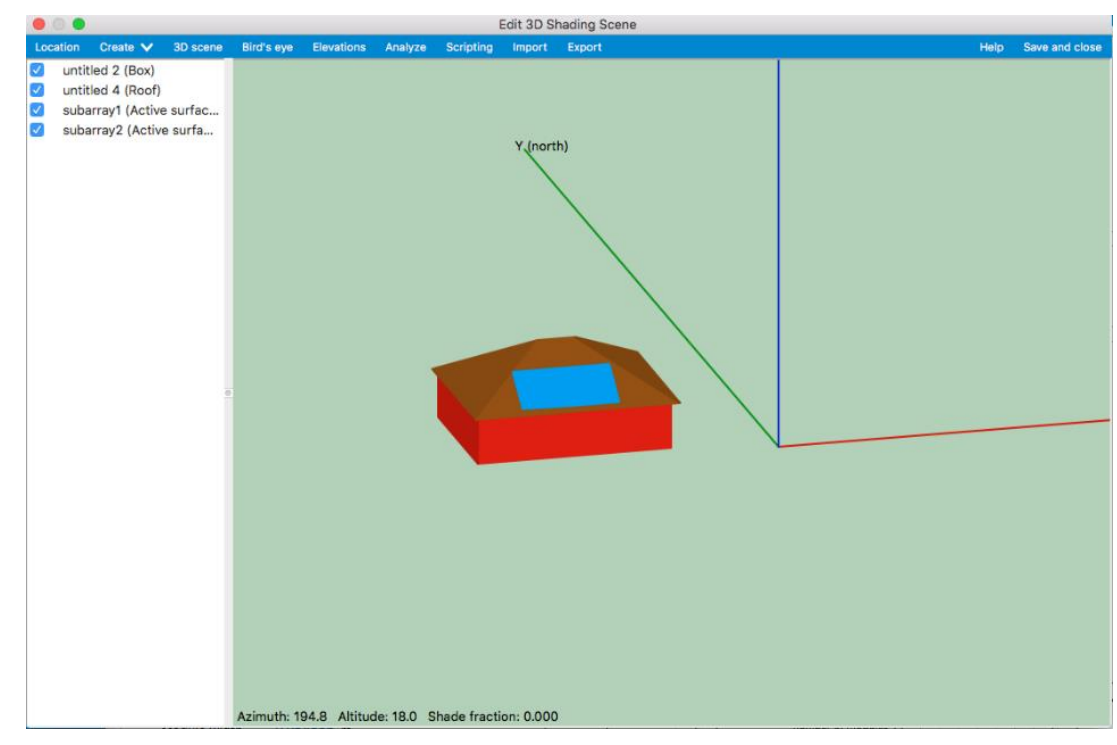

Figure 28. 3D drawing representing the case study area in SAM's $3 D$ shade calculator. 


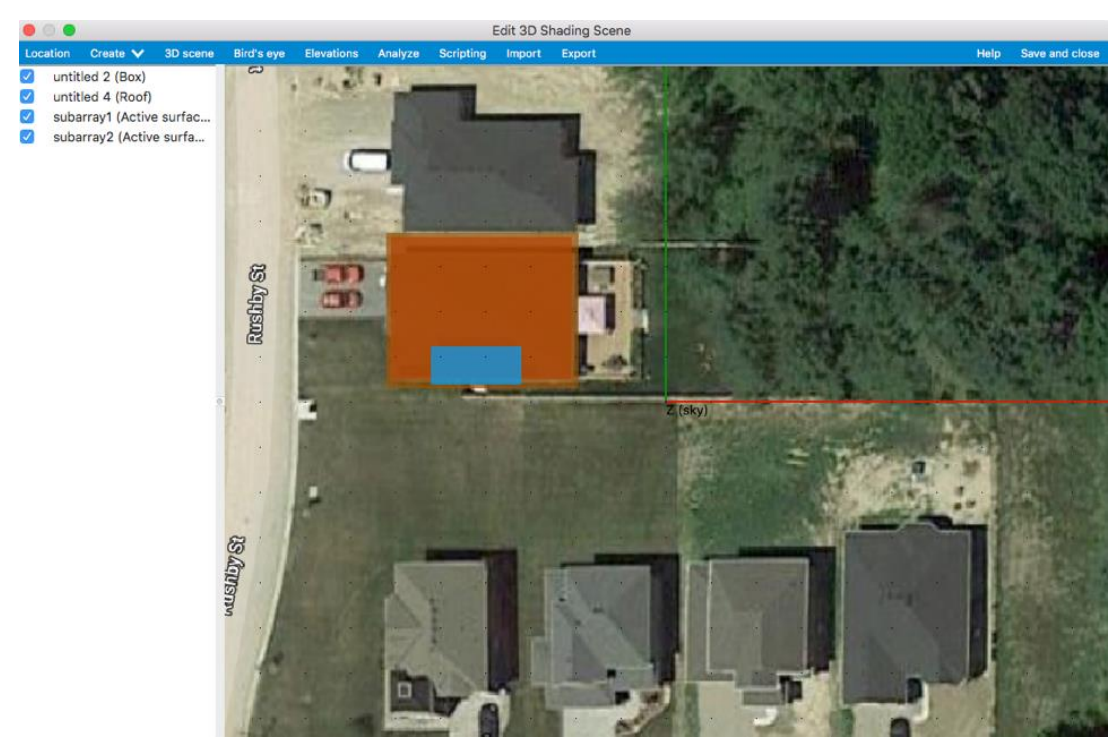

Figure 29. Bird's eye view of case study area with Google maps underlay.

(vi) Losses

This part of the simulation process takes into account the losses to the system due to soiling and electrical losses that the module and inverter models did not take into consideration such as irradiance losses, DC losses, AC losses, transmission and transformer losses. The default values were used for simulation as recommended by SAM. Additionally, the DC optimizers losses option was selected in order to account for the usage of the SolarEdge P300 optimizer in the case study area.

(vii) Simulations

After defining all the system information and parameters in steps (i)-(vi), simulations were performed for the case study area. Intermediate simulations were performed to test the decomposition models in SAM to see what effect it had on the final results. The three different combinations DNI \& DHI, DNI \& GHI, and GHI \& DHI were simulated under the Perez model and the annual results on PV generation were 11,305 kWh, 11,298 kWh, and 11, $296 \mathrm{kWh}$, respectively. Since there was not much difference on the annual generation, the recommended default DNI \& DHI decomposition model was chosen. Finally, a total of three simulations were performed in SAM using the three different transposition models Isotropic, HDKR, and Perez. 


\subsubsection{Physical Functions and Capabilities of the Software Tools}

This section will be discussing the physical functions and capabilities of the software tools HelioScope, PVsyst, PV*SOL, Archelios, EnergyPlus, and SAM. A list of their physical functions and capabilities were created for assessment, based upon the analysis that was performed in Section 4.2.2. The list is as follows:

1) System requirements and availability

2) Documentation and maintenance

3) Weather data

4) Solar radiation models

5) 3D modeling capabilities

6) Shade modeling

7) Maximum modeling capabilities

8) PV and inverter component databases

9) PV performance model

10) Inverter modeling

11) Modeling of system losses

12) Cost of software tool

13) Report and analysis

14) User-friendliness and level of expertise

15) Modeling flexibility

\subsubsection{System Requirements and Availability}

PVsyst, HelioScope, PV*SOL and Archelios are all proprietary software tools and require the purchase of a license. Academic and educational licenses are available at a discounted rate upon request for these software tools. HelioScope and Archelios are both available as an online application and requires the usage of an internet browser. A paid subscription account and internet connection is required.

PVsyst is available as application for Windows, supported by Microsoft Vista/7/8/10 32/64 bit. Other requirements that are recommended for the software tool include:

- At least $1 \mathrm{~GB}$ of RAM 
- At least $1 \mathrm{~GB}$ of free hard drive space

- Min screen resolution of 800 x 600 pixels (SVGA)

- .NET 4.5 framework (installed by Windows Update)

- Graphics card supporting OpenGl 2.0 or higher

$\mathrm{PV} * \mathrm{SOL}$ is a commercially available software tool for Windows by Valentin Energy Software in Germany. The recommended system requirements for $\mathrm{PV} * \mathrm{SOL}$ premium are:

- Internet connection

- Processor Intel i3 or equivalent

- RAM: 4GB

- Free diskspace: $850 \mathrm{MB}$

- Monitor resolution: min. 1024 x 768 px

- Operating systems: Windows 7, Windows 8.1, Windows 10

- Graphics: DirectX - compatible (min Version 9.0c), 1GB, OpenGL - support

- Other : Microsoft.NET Framework 4.7.2 Redistributable package

SAM is available for free, open-source desktop application for Windows, Linux and Mac OS. It is also available as an application programming interface (API) with a set of programming tools in a software development kit (SDK), and as a set of documented open source $\mathrm{C}++$ code repositories.

EnergyPlus is available for free as an open-source cross platform simulation tool, and is available for Windows, Mac OS X, and Linux operating systems. It was developed by the United States Department of Energy in the 1970s and receives inputs and outputs as text files as a command line interface. It is compatible with several graphical interfaces such as DesignBuilder and OpenStudio. System requirements vary depending on the type of graphical interface chosen for visual representation.

\subsubsection{Documentation \& Maintenance}

Detailed documentation for the calculation methods and models were available for five out of the six software tools analyzed: HelioScope, EnergyPlus, SAM, PVsyst, and PV*SOL. The documentation was very helpful and provided transparency to the theories and calculation 
methods used in the simulation procedures. Archelios Pro was the only software tool that did not have much documentation and was poorly translated from French. Customer service had to be frequently contacted regarding the modeling capabilities of Archelios and the parameters used.

PVsyst, EnergyPlus and SAM have very active online forums, and posts are replied to within a timely manner. It is a good place for learning, and for receiving support from the online user community as well as the developers of the software platform. HelioScope and PVSOL do not have a large active forum, however, active online help support is available from the company.

Since both SAM and EnergyPlus are both open source software tools, their documentation and source code are available to the public. This allows for researchers or developers who want to expand on their capabilities or functionality the opportunity to do so. All software tools show historical release notes for updates and revisions multiple times a year, except for Archelios.

\subsubsection{Weather data}

All the software tools analyzed came with different types of meteorological databases and native file compatibility formats. The summary of their weather data functions are as follows:

\section{(i) Helioscope}

HelioScope has various meteorological sources and options available for the user to select from. It has an online weather database that automatically chooses the closest weather data for the project location using Meteonorm weather data. There is an option where one can upload their own weather data information, as well as see what sources of data HelioScope currently has in their weather database. Currently, the weather data file formats that are accepted for import are: EPW, TMY2, TMY3, and Prospector formats.

\section{(ii) PVsyst}

The default meteorological data source used in PVsyst are from Meteonorm and NASASSE. PVsyst also has a large database system in which you can import various types of weather data. It covers an extensive amount of various sources of weather files. Meteorological data sources covered by PVsyst include: (3Tier) Vaisala, Canadian EPW, Explorador Solar, 
Meteonorm, NREL TMY2/TMY3, NREL NSRDB, PVGIS, Satellight, Soda-Helioclim3, Solar Anywhere (SUNY model), Solargis, Solar Prospector, Vortex Solar, NASA SSE, RETScreen and WRDC (World Radiation Data Center). These meteorological source files can be imported directly into PVsyst using the meteo database. PVsyst is very versatile, where one can import (or input) their own created meteo data, as well as personal data collected in many different formats. There is a weather tool in PV syst that helps with the programming and syntax for the weather files. In order to create your own weather file, PVsyst requires the following hourly meteorological data: horizontal global irradiance (GHI), ambient temperature, horizontal diffuse irradiance, and wind velocity.

(iii) $P V^{*} S O L$

The default weather data in PVSOL are provided by Meteonorm for most locations across the globe. Locations that are not included are interpolated using satellite and adjacent ground stations. PV*SOL also has a weather module built in called MeteoSyn that provides sitedependent climate data. This is the module that offers the user the ability to extract the climate data from an interactive online map for locations not available on the default list selection. PVSOL weather file formats that are valid are: DWD (*.xml), Meteonorm (*.dat), and WBV (*.wbv). In order to use your own weather data, it has to be imported in (*.dat) format and it needs to contain hourly information for the following four parameters: ambient temperature $\left({ }^{\circ} \mathrm{C}\right)$, global radiation horizontal $\left(\mathrm{Wh} / \mathrm{m}^{2}\right)$, wind velocity $(\mathrm{m} / \mathrm{s})$ and relative humidity $(\%)$.

(iv) Archelios

For weather data, Archelios will use the nearest reference station based upon Meteonorm data from the Archelios database. The user can choose to modify the meteo station if desired, or import weather file in the following formats: Archelios meteo (csv), PVGis (txt), PVGis (web service), PVGis (TMY), NREL (TMY3), Helioclim (TMY3), Helioclim PVsyst (TMY), Solargis (TMY), and Solargis PVsyst (TMY). In order to create a custom weather data set, the user needs to provide the monthly mean data in Archelios meteo csv format (note that you cannot import hourly csv data): direct irradiance $\left(\mathrm{kWh} / \mathrm{m}^{2}\right)$, diffuse irradiance $\left(\mathrm{kWh} / \mathrm{m}^{2}\right)$, solar fraction $(\%)$, wind speed $(\mathrm{m} / \mathrm{s})$, air temperature $\left({ }^{\circ} \mathrm{C}\right)$, and link trouble (link turbity). This format is unlike other typical weather importation formats as it requires two additional variables solar fraction and link 
trouble. There was also no definition available from Archelios help menu for the last two parameters, leading to confusion and uncertainty of the meanings.

(v) EnergyPlus

EnergyPlus has weather data available for more than 2100 locations around the world. Out of all the simulation programs EnergyPlus has the most extensive weather data available. EnergyPlus is compatible with generally any formats of TMY weather data formats such as TMY2, TMY3, and EPW formats. EnergyPlus also comes with a weather converter program, to help with the conversion of weather data formats such as IWEC, Blast etc. Users can create their own EPW CSV format weather file for simulation, however, this requires an extensive amount of weather data compared to the rest of the PV simulation software programs. The data required include: dry bulb temperature $\left({ }^{\circ} \mathrm{C}\right)$, dew point temperature $\left({ }^{\circ} \mathrm{C}\right)$, relative humidity $(\%)$, atmospheric pressure $(\mathrm{Pa})$, horizontal infrared radiation intensity from sky $\left(\mathrm{Wh} / \mathrm{m}^{2}\right)$, direct normal radiation $\left(\mathrm{Wh} / \mathrm{m}^{2}\right)$, diffuse horizontal radiation $\left(\mathrm{Wh} / \mathrm{m}^{2}\right)$, wind direction (degrees), wind speed $(\mathrm{m} / \mathrm{s})$, and liquid precipitation depth.

(vi) $S A M$

SAM has a Solar Resource Library that is available for download and custom weather data can be incorporated into it. The default location where SAM downloads weather data is from the NREL National Solar Radiation Database (NSRDB). For locations that are not in the NSRDB or if one wants to use their own weather data, the data needs to be imported into a compatible format with SAM. SAM uses a CSV comma-separated text format for solar resource data in order to perform its solar performance model calculations. The CSV supports hourly and sub-hourly data with up to a one-minute resolution. SAM can read weather data in TMY3, TMY2, and EPW formats. SAM also has a 'Solar Resource File Converter' macro that can convert these files into the SAM CSV format. Table 5 shows the weather data variables required for creating your own data for the three different solar performance models in SAM (since each model requires different weather data elements). 
Table 5. Weather data information required for creating own solar resource data set in SAM.

\begin{tabular}{|l|c|c|c|}
\hline $\begin{array}{l}\text { Weather Data } \\
\text { Element }\end{array}$ & PV Detailed & PVWatts & $\begin{array}{l}\text { High Concentration } \\
\text { PV }\end{array}$ \\
\hline $\begin{array}{l}\text { Latitude (decimal } \\
\text { degrees) }\end{array}$ & $\bullet$ & $\bullet$ & $\bullet$ \\
\hline $\begin{array}{l}\text { Longitude (decimal } \\
\text { degrees) }\end{array}$ & $\bullet$ & $\bullet$ & $\bullet$ \\
\hline $\begin{array}{l}\text { Elevation above sea } \\
\text { level (m) }\end{array}$ & $\bullet$ & & $\bullet$ \\
\hline Hour of the day & $\bullet$ & $\bullet$ & $\bullet$ \\
\hline $\begin{array}{l}\text { Diffuse horizontal } \\
\left.\text { irradiance (W/m }{ }^{2}\right)\end{array}$ & $\bullet$ & $\bullet$ & $\bullet$ \\
\hline $\begin{array}{l}\text { Direct normal } \\
\left.\text { irradiance (W/m }{ }^{2}\right)\end{array}$ & $\bullet$ & $\bullet$ & $\bullet$ \\
\hline $\begin{array}{l}\text { Global horizontal } \\
\left.\text { irradiance (W/m }{ }^{2}\right)\end{array}$ & $\bullet$ & & $\bullet$ \\
\hline Albedo & $\bullet$ & $\bullet$ & \\
\hline $\begin{array}{l}\text { Dry bulb temperature } \\
\left({ }^{\circ} \text { C) }\right.\end{array}$ & $\bullet$ & $\bullet$ & \\
\hline Wind velocity (m/s) & $\bullet$ & & \\
\hline Snow depth & & & \\
\hline
\end{tabular}

\subsubsection{Solar Radiation Models}

Solar radiation modeling is an important option to have in the software tools as depending on the quality of the weather data, it can affect your output. Solar radiation modeling options were available for all the software tools except for Archelios. Archelios uses the Perez model for the transposition of solar irradiation to the POA. The options in the other software tools are as follows: 


\section{(i) HelioScope}

HelioScope offers the choice between using the Perez or Hay model. The default model is Perez. In general, the Perez model is recommended for high quality weather files, and the Hay model is better for lower quality weather data.

(ii) PVsyst

PVsyst has two options for solar radiation transposition models, the Hay model and the Perez-Ineichen et al. (1990) model. Prior to PVsyst version 5, the default model was the Hay model, however, according to studies done by Ineichen (2011) the Perez model was found to be slightly more accurate. Thus, the Perez model is now the default model in PVsyst. The Hay's model is a classic and robust model that produces favorable results even when the knowledge of the diffuse irradiation is not perfect. The Perez-Ineichen model (Perez et al., 1990) is a more sophisticated model than the Hay's model, and it requires accurately measured horizontal data (PVsyst, 2019).

\section{(iii) $P V^{*} S O L$}

Out of all the software tools evaluated, PV*SOL had the most options available for the modeling of solar radiation. It offered eight options for decomposition models for the breaking down of GHI into its direct and diffuse components and five models for the transposition of irradiation onto the inclined plane. The eight decomposition models are: (1) Hofmann, (2) Reindl (reduced), (3) Orgill \& Hollands, (4) Erbs, Klein \& Duffie, (5) Boland, Ridley \& Laurent, (6) Boland, Ridley \& Laurent (2010), (7) Perez \& Ineichen, and (8) Skarveit. The default diffuse irradiation model used was the Hofmann model and that was the model that was used for all the simulations. The five transposition model options are: (1) Hay \& Davies, (2) Liu \& Jordan, (3) Klucher, (4) Perez, and (5) Reindl. The Hay \& Davies is the default parameter set for simulation.

(iv) EnergyPlus

EnergyPlus, much like Archelios, only uses the Perez transposition model. However, because EnergyPlus is a building energy simulation program, it does offer a few other options for 
modeling design day solar radiation calculations such as the ASHRAE Clear Sky Solar Model, ASHRAE Revised Clear Sky Model (“Tau Model”), and the Zhang-Huang Solar Model.

(v) $S A M$

There are three options for transposition models in SAM and they are: (1) Isotropic, (2) HDKR, and (3) Perez (default) sky diffuse models. Weather file irradiance data can also be calculated in SAM in various combinations and they are: DNI and DHI, DNI and GHI, GHI and DHI, POA from reference cell, and POA from pyranometer.

\subsubsection{3D Modeling Capabilities}

3D modeling capabilities are an important functionality for the software tools to have in order to create the exact 3D geometry of the case study area for the performance of detailed analysis on the impacts of surrounding buildings and obstructions. That being said, the capabilities for modeling exact geometries vary depending on the software tools used. The 3D modeling capabilities of the analyzed software tools are highlighted as follows:

\section{(i) HelioScope}

Although HelioScope is capable of modeling 3D geometries, the level of detail of the drawings are rather simplistic as compared to commonly used CAD software tools. In HelioScope, a 3D drawing is created from satellite imagery of buildings. The rooftop or footprint of the building is traced manually, and then extruded to the desired height, orientation and slope. There is no option to import any 3D files at this point, however, the created 3D file can be exported in DXF format for editing in other CAD based software.

\section{(ii) PVsyst}

PVsyst uses a 'near shadings' 3D shade constructor tool for 3D modeling. Users can create detailed drawings of the case study area or choose to import an existing CAD file. 3D Files of the types 3DS and DAE are compatible. This allows for the creation of the exact 3D geometry of your case study area and to analyze the impacts of surrounding obstructions. The ability to import shading scenes from other CAD based software was recently added in version 
6.60. Although PVsyst has the ability to handle large and detailed geometries, it is recommended that very detailed scenes to not be used, as this will make calculation time grow exponentially.

(iii) $P V * S O L$

The $3 \mathrm{D}$ visualization mode in $\mathrm{PV} * \mathrm{SOL}$ is only available in the Premium version and it is the most visually appealing out of all the software tools analyzed. It has the ability to create highly detailed drawings, surrounding objects and buildings can easily be modeled with realistic rendering. It is beneficial for providing detailed information on shadows cast at different times of the day for shading analysis to see which configurations would be more optimal. The detailed shading analysis is performed on individual modules, and the effect on the power optimization on the system yield can be visualized by the interface.

Satellite imagery can also be uploaded to the 3D drawing as well. The ability to import 3D data was made available in the Premium 2018 release and a wide variety of 3D file formats are supported. Supported formats include: DirectX X (*.X), Wavefront Object (*.obj), Collada (*.dae), Blender 3D (*.blend), 3ds MAX 3DS (*.3ds), 3ds MAX ASE (*.ase), LightWave (*.lwo), Stanford Polygon Library (*.ply), Stereolithography (*.stl), and Milkshape 3D (*.ms3d).

(iv) Archelios

Archelios does not have 3D modeling capabilities on its own. It requires the usage of third-party software to perform 3D simulations. It uses Trimble SketchUp with Archelios downloaded as a plugin. The information from the shading analysis performed is then exported to the main Archelios web application.

\section{(v) EnergyPlus}

EnergyPlus on its own is a command line simulation engine that reads input and writes output to text files and does not have a graphical user interface. Third-party software is required for 3D modeling capabilities. A lot of energy modeling software tools support data exchange with EnergyPlus and numerous graphical interfaces have become available for 3D modeling using EnergyPlus. Commonly used software tools that are use or are compatible with the 
EnergyPlus simulation engine include Trimble SketchUp, Euclid, DesignBuilder, HoneyBee, and so forth.

(vi) $S A M$

Out of all the software tools analyzed, SAM had the least visually appealing 3D modeling capabilities. SAM uses a 3D Shade Calculator to store and read 3D scene data in SAM native .s3d format. The importation of CAD based data is not possible as it cannot read files in other formats. The drawing capabilities are extremely limited in SAM in which only active surfaces (PV array surface) and predefined shading objects can be drawn. The predefined objects that can be drawn in SAM are a tree, cylinder, rooftop and rectangle. There is no way to modify the predefined geometries, and measurements cannot be drawn precisely. The shading scene is meant to be for proportional purposes, and not drawn 100\% to scale. A user can add an optional satellite imagery from online maps as an underlay to the scene if desired. Figure 30 provides an example of all the objects that one can draw in SAM.

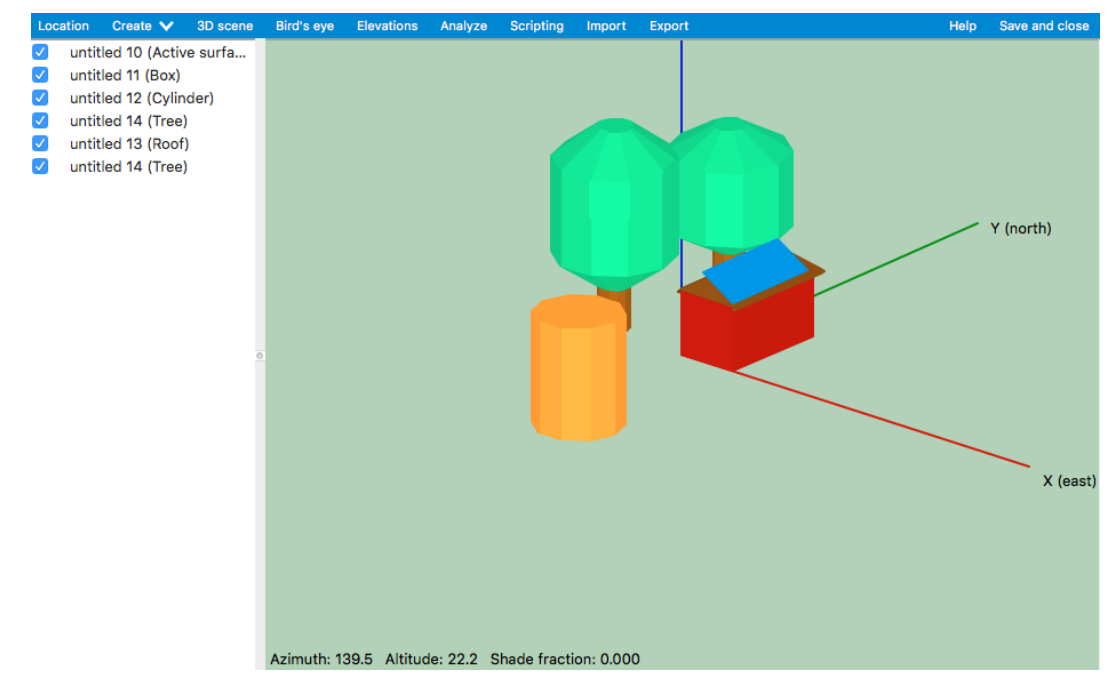

Figure 30. SAM 3D Shading Scene calculator depicting drawing capabilities.

\subsubsection{Shade Modeling}

The accurate modeling of shading is very important for a PV system as it effects the amount of beam and diffuse irradiance received on the PV system, and can have a significant effect on electrical losses and hence the total electrical output of the system. Estimation of the 
shadows falling on the PV system are simulated in the 3D modeling components of all the software tools, and determination of the shadows are carried out in a geometric analytical manner. Hence, having a 3D model is very important as it is acalculation of the shadows. The following highlights the shade modeling functionality of the software tools:

\section{(i) HelioScope}

There are three main sources of shading that HelioScope takes into consideration when performing calculations (Folsom Labs, 2018):

i) Row-to-row shading - this calculates the shading losses caused by interrow shading for arrays that are installed on a tilt

ii) Obstruction shading (from keepouts) - Shading losses are applied based on the height and proximity of the surrounding obstructions and are applied on each module in the array, for each hour of the year

iii) Horizon files - horizon files can be used which are basically files created with third party software (such as Solmetric SunEye) which are a collection of azimuth/elevation points that define the horizon around the array. These can be uploaded into the shading tab.

(ii) PVsyst

In PVsyst, it models shadings in two main ways distinguishing between far shadings and near shadings. Far shadings are described by the horizon of far shadings and are suited for treating the horizon line. The horizon line is a broken line superimposed on the sun path diagram with numerous height/azimuth points. When the sun is below the horizon line, there will be no shading loss. Horizon profiles can be taken from other software tools. For the treatment of near shadings, PVsyst is referring to shadings that are produced by nearby objects onto the PV array. The treatment of near shadings uses the 3D modeling editor in PVsyst in order to simulate the PV system and its full environment. The shading computations are computed every hour, and are applied to the beam, diffuse and albedo components.

(iii) $P V * S O L$ 
PVSOL's 3D visualization mode provides very detailed information on the shadows cast at various times of the day and year for shading analysis to see which configurations would be more optimal. The detailed shading analysis is performed on individual modules, and a shade frequency calculation can be performed to see which PV modules are heavily shaded, so that designs can be changed on the fly. PVSOL can also model shading using the horizon line for far shadings and is compatible with horizon file imports from other software. An annual percentage value for far shading losses can also be entered.

\section{(iv) Archelios}

Archelios has an irradiation computation function that calculates the amount of irradiation level for each PV module in the system. This function visually shows the amount of irradiation received on each PV module, and can filter the modules that are shaded or receive less than a specified minimum amount of irradiation per year, based upon the surrounding shading objects and obstructions. Archelios Pro automatically calculates the horizon line from NASA data and can also accept horizon mask import files. Shading losses may be different at optimal orientation and tilt.

\section{(v) EnergyPlus}

EnergyPlus by default excludes other buildings on the site from analysis. It only takes into consideration the current building geometry; however, it can be told to include all other buildings on the site to be modeled as shading obstructions. All external surfaces in buildings other than the current one being modelled are modeled as shading surfaces. Furthermore, there is the additional option to model all external reflections and shading of ground reflected solar. If one or more of these buildings are complicated or large, this could generate a lot of shading elements and the simulations could be extremely time-consuming. Note that EnergyPlus is the only software tool analyzed that does not take into consideration far shadings from the horizon line.

(vi) SAM

In general, SAM can model the impact of shading on each subarray caused by external shading, self-shading and snow cover. The shading loss represents the obstruction of beam 
irradiance on the array at each point in time. Losses are modeled as linear with shaded area and SAM may tend to underpredict losses due to shading when nonlinear mismatch losses are present. External shading refers to the shading of beam and diffuse incident irradiance by surrounding objects and buildings and are applied on top of any additional soiling losses. External shading can be estimated in two ways, through the usage of shade loss tables, or the 3D Shade calculator.

Shading loss tables can be calculated in a software tool like PVsyst and then imported into SAM, or even onsite with survey tools such as Solmetric SunEye or SolarPathfinder. One can also import table of shading losses by month and hour of day, table of shading losses by solar azimuth and altitude, hourly beam irradiance shading loss percentages, or constant diffuse shading loss. This can be customized for each subarray, making it very detailed for shading loss analysis.

The 3D Shade Calculator enables the user to create a 3D scene of the project and will calculate a month by month-by-hour shading loss table of beam irradiance shade loss percentages and a sky diffuse loss percentage. There is an option to model self-shading which is caused by interrow shading of the modules within a subarray. In general, this is used for larger commercial rooftop and utility scale installations arranged in a regular pattern to determine when one row shades the next and is applicable to fixed tilt or one axis tracking arrays.

SAM has a unique snow losses modeling option that estimates the loss in system output during the times when the array is covered in snow. This requires the knowledge of snow depth information from the weather file and for time steps with snow, it calculates the percentage of the PV array that is covered with snow based on the tilt, POA irradiance, and ambient temperature. It assumes that the array is completely covered in snow when there is snowfall, and that the snow slides off the array as a function of increasing ambient temperature.

\subsubsection{Maximum Modeling Capabilities}

Software tools all vary in degrees of modeling complexity and capabilities. Due to the inherent differences in each software tool, it is important to determine the maximum modeling capabilities and if there are any restrictions to the modeling of the scene complexity. This section will discuss any scene complexity and modeling restrictions that the software tools were discovered to have. 


\section{(i) HelioScope}

The maximum recommended system size design for a layout in HelioScope is 5MW, however it is possible to push the limits of the software and to create a design that exceeds 5MW. HelioScope will simulate the production of arrays up to $15 \mathrm{MW}$ in total size, however, it is not recommended. Simulations this large would affect simulation speed, CAD exports failing and layout images not rendering. It is recommended to break larger designs down into easily manageable blocks.

(ii) PVsyst

There is no limitation to the PV system maximum size in PVsyst, however, it can only model a maximum of 8 different subsystems (subarrays). PVsyst can simulate systems that are comprised of more than one size and/or type of inverter, as well as arrays with different tilts and azimuths connected to one single inverter. Note that although PVsyst has the ability to handle large and detailed geometries, the more complicated the 3D scene, the longer it will take to perform the simulations on an exponential basis.

(iii) $P V^{*} S O L$

In PV*SOL Premium 3D planning mode, the restrictions to modeling are the number of modules that the system can model. There is no $\mathrm{kW}$ size restriction to the PV system, or restriction to the complexity of the $3 \mathrm{D}$ scene. The number of modules is limited to 7,500 for elevated systems and 10,000 modules for roof-parallel systems. In 2D planning, you can simulate up to 100,000 modules per module area and the number of module areas is unlimited.

\section{(iv) Archelios}

Archelios has a modeling size restriction of up to 50kWp, however, Archelios Pro version allows the user to model projects greater than $50 \mathrm{kWp}$. There is no size restriction for modeling in Archelios Pro, however, the browser can have some memory issues and calculation times can take a very long time.

(v) EnergyPlus 
There is no maximum system size that is stated in EnergyPlus, however, simulations may take a very long time. Note that when using EnergyPlus in DesignBuilder, it does have a limitation to a restriction of a maximum of thirty generators that can be added to each electric load centre, which means that you can model a maximum of 30 active PV areas, in one simulation. Hence it does not make sense to model each panel individually (since more than likely you will have more than $30 \mathrm{PV}$ panels) and it is easier to model if you group them together.

\section{(vi) $S A M$}

There is no limit to the number of active surfaces and shading objects that you can draw in the 3D Shade Calculator scene, however, there is a limitation to how many PV systems SAM can analyze in one simulation. SAM can model PV systems with up to four strings, and its partial shading model can handle subarrays with up to eight strings, so the 3D Shade Calculator allows you to assign active surfaces to up to four subarrays and eight strings (a maximum of 32). The calculator generates shade data for each string in each subarray. Also, SAM can only model systems with one type of module, and one type of inverter. You cannot use subarrays to model a system that combines different types of modules or inverters.

\subsubsection{PV and Inverter Component Database}

In general, most solar simulation software tools offer a large database of information on thousands of PV models and inverters for most manufacturers and models. These are based upon manufacturer specifications or datasheets and are maintained on a regular basis by the software companies. Component databases are generally stored in *.PAN format and are compatible for import with other solar simulation software tools. Users of a software tool can also add their own PV or inverter data, if it the data is not available in a particular database.

All the software tools analyzed contained a large regularly maintained PV and inverter component database, except for EnergyPlus. This is because EnergyPlus is a whole building energy simulation software tool, and not designed specifically for PV modeling.

PV*SOL has the most comprehensive database out of all the software tools evaluated. PV*SOL also has a database for battery systems, generators, electric vehicles, and even FIT 
programs for many countries (Brazil, Germany, France, Italy, Netherlands, Austria, Spain, United States, and United Kingdom).

\subsubsection{PV Modeling}

PV performance models help simulate the electrical behaviour of a PV module given by the specific irradiance and temperature of that module. All the software tools analyzed use the single-diode model to characterize module performance at STC and an additional set of parameters to predict performance at changing irradiance and temperature conditions. The PV performance are calculated based upon the module performance information within their database that are characterization files generally provided by the manufacturer. This contains information from the manufacturer data sheet for about the solar panels such as shunt circuit current, open circuit voltage, voltage at maximum power, temperature coefficients for short and open circuit voltage, number of cells per module, cell temperature at NOCT conditions and module area.

EnergyPlus and SAM were the only two software tools that offered a few other options to model PV performance. There are two additional options on top of the single diode model for modeling PV performance in EnergyPlus. The two additional models are the simple PV efficiency model and the Sandia model. The simple PV model requires the efficiency with which the PV panels convert incident solar radiation to electricity and does not require arrays of specific modules to be defined. This model is best suited when the exact specifications of the solar panels are unknown. The Sandia model is based on the testing data performed at Sandia National Lab. This object uses specific performance input data needed for specific make and model types of PV panels using the empirical coefficients derived at Sandia and requires a lot of extensive measurements and data. This data is available only for specific modules that have been tested and published by Sandia.

SAM was the software tool that had the most options for PV modeling. It had a total of five options available:

1) Simple efficiency module model - The simplest method of the options, and the least accurate. It requires only a few parameters. 
2) Single-diode model - CEC performance model with module database (default) Calculates the performance based upon data stored in a library for thousands of commercial PV modules. This is a single-diode model from the California Energy Commission an extension of the University of Wisconsin 5 parameter model.

3) Single-diode model - CEC performance model with user enter specifications - Same as the above, except it is used based upon user enter data

4) Sandia PV Array Performance Model with module database - Based upon empirically derived test data by Sandia National Laboratories (only if available).

5) IEC61853 Single diode model - This method is based upon detailed test data and calculations from the International Electrotechnical Commission (IEC) power and rating standard.

\subsubsection{Inverter Modeling}

Inverter models are used to calculate DC to AC conversion efficiency under the assumption that DC power input to the inverter is equal to the DC output of the PV array. All software tools model the inverter based upon the efficiency curve of the inverter which can be determined from the manufacturer. All software tools performed this modeling based upon the specified inverter (from the database or user uploaded) except for EnergyPlus.

SAM provided a few options for the modeling of the performance of the inverter. The four ways mentioned are as follows:

(1) Inverter CEC database - The default method in SAM. This calculates the AC output using parameters from the SAM CEC inverter database with the Sandia inverter model. This method is automatically chosen when an inverter is used from the database.

(2) Inverter datasheet - Similar to the above method, except it is used for inverters that are not specified in the SAM CEC database. Calculations are performed based on the inputted.

(3) Inverter Part load Curve - This model can be used when the part load efficiency values for an inverter are known.

(4) Inverter CEC Coefficient Generator - If inverter test data is available, this method can be used to generate coefficients for the Sandia inverter model. 
In EnergyPlus, there are three types of inverter models available:

(1) Simple - efficiency is constant and inputted by the user

(2) Look up table - the efficiency is calculated using linear interpolation

(3) Function of power - the efficiency is calculated using a single-variable curve object.

\subsubsection{Modeling of System Losses}

The capabilities of the software tools pertaining to the modeling of system losses is very important as it can lead to differences in total system yield. PV systems are susceptible to losses caused by dust or snow cover, electrical losses, ageing, wiring losses, etc. The level of detail in which system losses (derates) are modeled vary per software. Some software tools may have the capability to model many system loss factors, whereas others only a few. The factors taken into

consideration may vary as well. Note that not all power losses that occur in a system are listed as options that are modifiable by the user, for example some such as thermal losses due to system configurations are modeled explicitly (Guittet \& Freeman, 2018). The differences between the many various expected inputs may provide an indication of the complexity of the tools as well as aspects of the software tools modeling specialities (Freeman et al., 2014). Here is a summary of the various system derate loss factors that are accounted for in each of the software tools and their default values:

\section{(i) HelioScope}

Out of all the software tools analyzed, HelioScope had the least amount of control over the number of parameters that the user can control for modeling system losses. During the simulation procedure, the default derate factors that the user can modify are:

1. Monthly soiling derate $=2 \%$

2. AC system derate $=0.5 \%$

\section{(ii) PVsyst}

Out of all the software tools, PVsyst has the most detailed system loss modeling capabilities and options. In the detailed losses simulation parameters, many detailed customizations can be adjusted if exact numbers are known for the losses. PVsyst has a lot of various loss calculations available that take into consideration: thermal parameter, ohmic losses, module quality-LID- 
mismatch, soiling loss, IAM losses, ageing, spectral correction, auxiliaries consumption, external transformer and system unavailability losses. PVsyst calculates the IAM losses, thermal losses and standard NOCT factors based upon your PV technology. It can also calculation degradation in the system over time, the default setting is turned off. Detailed loss analysis charts are provided at the end of the simulation in a detailed report as well. Default loss values recommended in PVsyst include:

1. DC circuit ohmic losses $=1.5 \%$

2. Module efficiency loss $=0.5 \%$

3. $\mathrm{LID}=$ light induced degradation $=2 \%$

4. Module mismatch losses- power loss at MPP $=0 \%$

5. Module mismatch losses - loss when running at fixed voltage $=0 \%$

6. Strings voltage mismatch- power loss at MPP $=0 \%$

7. Yearly soiling loss factor $-3 \%$ (can define monthly values as well)

8. Unavailability of the system - unavailability time fraction $2 \%$, for a duration of 7.30 days/year, for 3 periods

9. Albedo (monthly) $=20 \%$

(iii) $P V^{*} S O L$

PV*SOL default System derates are as follows:

1. Losses due to deviation from standard spectrum $=1 \%$

2. Power losses resulting from a drop in voltage at the bypass diodes $=0.5 \%$

3. Power losses resulting from mismatching or reduced yield $=2 \%$

4. Ground Reflection (albedo) $=20 \%$ (annual or monthly)

5. Losses due to soiling $=0 \%$ (annual or monthly)

(iv) Archelios

The follow parameters are the default system losses and their values that Archelios takes into consideration:

1. Monthly albedo values $=20 \%$

2. Losses in $\mathrm{DC}$ wires $-\mathrm{STC}=1 \%$

3. Losses in $\mathrm{AC}$ wires $-\mathrm{STC}=1 \%$ 
4. Module dirt losses $=2 \%$

5. Module aging $=0.5 \% /$ year

6. $\mathrm{LID}=0 \%$

7. Mismatch $=2 \%$

(v) EnergyPlus

EnergyPlus is not a PV specific modeling software tool, so specific PV loss parameters do not exist. However, due to the modeling flexibility of EnergyPlus you can create schedules for the PV system to take into consideration system downtime.

(vi) SAM

SAM has quite a few options for the control of the various factors in the modeling of the system losses. The default values in SAM are:

1. Soiling loss annual $=5 \%$

2. $\mathrm{AC}$ wiring $=1 \%$

3. Transformer losses $=0 \%$

4. Transmission losses $=0 \%$

5. Monthly albedo default $=20 \%$

6. Curtailment and availability $=0 \%$

Just like PVsyst, it is also possible to model system output losses due to system unavailability to represent system outages or other events, and these options can be specified. By default, it is not modeled. A unique feature to SAM is that it offers different default DC losses values depending on the type of inverter used: central inverter, microinverter or DC optimizer. These values are summarized in Table 6. 
Table 6. Summary of DC loss values in SAM for central inverters, microinverters and DC optimizers

\begin{tabular}{|c|c|c|c|}
\hline & Central inverter & Microinverter & DC optimizers \\
\hline Module mismatch & 2 & 0 & 0 \\
\hline Diodes and connections & 0.5 & 0.5 & 0.5 \\
\hline DC wiring & 2 & 2 & 0 \\
\hline Tracking error & 0 & 0 & 0 \\
\hline Nameplate & 0 & 0 & 1 \\
\hline DC power optimizer loss & 0 & 0 & 2 \\
\hline
\end{tabular}

\subsubsection{Cost of Software Tools}

The cost of the software tools was an important criteria to consider, as it was important that the usage and licensing of the software tool to be generally affordable.

\section{(i) HelioScope}

HelioScope is owned by Folsom Labs, and is available at a subscription fee (annually or monthly). It is available at $\$ 95$ per month or at a $17 \%$ discount of $\$ 950$ per year in USD. Academic and/or student pricing is available upon request.

(ii) PVsyst

PVsyst is a commercial software tool and requires an annual paid license. The first license is available at CHF 1300, with discounts applied for subsequent licenses at CHF 1000 for the second license, and CHF 700 for the third. Academic and/or student pricing is available upon request.

(iii) $P V * S O L$

PV*SOL premium software is licensed by Valentin Software and is available at an annual fee of $€ 1295$. Academic and/or student pricing is available upon request.

(iv) Archelios

Archelios Pro is sold by Trace Software and is available at a cost of $€ 990$ per year. Academic and/or student pricing is available upon request. 


\section{(v) EnergyPlus}

EnergyPlus is an opensource software tool and is available for free. However, depending on the third party software tool that is selected as a $3 \mathrm{D}$ simulation platform, there may be a licensing fee that the user may be subjected to. For example, DesignBuilder was selected for simulation in this dissertation, and it is a commercial software tool that requires an annual license. The annual licensing fee for DesignBuilder varies depending on the modules subscribed to and can range from $\$ 1399$ USD for the essentials package up to \$3199 USD per year for the Pro package. DesignBuilder offers an Academic and/or student pricing upon request.

(vi) SAM

SAM is an opensource software tool developed by NREL and is available free of charge.

\subsubsection{Report \& Analysis Output}

Report and analysis output options are an important capability of the software tools that need to be examined, as all software tools have different formatting and output options available. A sample report from each software tool generated can be viewed in the Appendices section at the end of the thesis. Note that there is no pdf report summary output from EnergyPlus, hence it is not included in the Appendices.

\section{(i) HelioScope}

After a simulation is finished in HelioScope, the reports tab shows all the Designs and Conditions that have been created for a project. In report view you can get monthly graphs of the electricity generated. Sources of system losses are displayed in a chart to easily visualize and optimize the losses your system receives. The user can choose between three different metrics shown on the reports and the choices are available in $\mathrm{kWh} / \mathrm{kWp}$, Performance Ratio, or Energy (total generation).

For further analysis, HelioScope allows for the export of the 3D drawings created to CAD format such as DXF with the satellite imagery as the base layer. This fully 3D CAD file contains the buildings designed, modules and wiring configurations. A final report can be generated in a nicely formatted pdf summarizing all the findings. For detailed analysis, hourly 
simulation data can be downloaded in CSV format. A sample pdf report generated in HelioScope can be viewed in Appendix B.

(ii) PVsyst

PVsyst has the capabilities to produce very detailed calculations and can output over 100 variables for one simulation on an hourly basis. The user has to define which hourly variables it wants calculated and outputted from the simulation, as depending on the project, it can be very computationally intensive. Detailed graphs and tables can be generated within PVsyst to analyze the simulation results as well as the weather data. It also performs P50/P90 calculations if the weather data is available. PVsyst is a very valuable tool when performing optimization calculations. Depending on the type of analysis, batch simulations can be performed for parametric analysis. PVsyst also produces a detailed pdf report documenting all the results with a detailed losses diagram. A sample pdf report generated in PVsyst is depicted in Appendix C.

(iii) $P V^{*} S O L$

PV*SOL's results page offers a variety of tables and figures to visualize and analyze the results of the simulation, and can be exported into various formats for data analysis. There is an option to export simulation data in hourly or minute time frame interval in csv format for analysis. Since PV*SOL is mainly used by industry professionals, the user can choose to produce a customized pdf presentation report for the client. A pdf report sample can be reviewed in Appendix D.

\section{(iv) Archelios}

Archelios has a few output options available. The results can be exported to other thirdparty software such as HOMER, and the 3D files can be exported in DXF CAD format. A pdf report can be generated summarizing the analysis, and an hourly CSV file can be exported. The hourly CSV files can be a bit confusing to read, as some of the parameters are not translated into English, and in its native language of French. A sample report format generated in Archelios can be reviewed in Appendix E. 


\section{(v) EnergyPlus}

EnergyPlus has the capability of producing very detailed reports for energy analysis, hence its popularity. The user has flexibility to command specific outputs and there are hundreds of different building energy simulation parameters that can be generated. EnergyPlus as mentioned before is a command line software tool that reads input and writes output to text files. The user has to tell EnergyPlus which variables to generate simulation results for (similar to PVsyst and SAM). A tool called EP-Launch could be used to help with visualizing some simple tabular results and files and EP-Compare for graphically comparing the results. Other third-party software can be helpful for visualizing reports and data from EnergyPlus, for example such as DesignBuilder. EnergyPlus does not generate a solar PV report in pdf format, like the other software tools.

(vi) $S A M$

SAM's results page is an interactive display that can create custom graphs and tables of the results generated. Similar to PVsyst and EnergyPlus, there are many simulation output variables to choose from. This needs to be defined by the user for the export to Excel or text and can be performed for up to 1-minute intervals. SAM can also perform some quick statistics on the data generated. A pdf is also created summarizing the results of the simulation. SAM can also produce a detailed losses summary just like PVsyst and it also allows for the parametric, stochastic and P50/P90 simulations for optimization of values and to examine the effects of uncertainty. A sample report generated in SAM can be reviewed in Appendix F.

\subsubsection{User-friendliness and level of expertise}

The level of user-friendliness and ease of use refers to the degree to which the software tool makes it easy for users to operate and control it (Miguel et al., 2014). The software tools analyzed in this thesis all had varying degrees of complexity and expertise required in order to perform a simulation. HelioScope was the most user-friendly software tool out of all the software tools evaluated, as it had the least number of parameters that needed to be inputted in order to perform a simulation. It also recommends how to divide your PV system into strings, and the number of modules per string, which greatly simplifies the modeling process. It also had extensive documentation and tutorials available, which eased the learning curve. 
Overall, the user-friendliness of PVsyst, PV*SOL, and SAM was found to be moderate, as they all have graphical user interfaces which makes it easy to perform simulations without the use of programming. Archelios wasn't very user-friendly as it was quite difficult to navigate its interface with little documentation. However, the level of expertise required for performing simulations in PVsyst, PV*SOL, Archelios, and SAM was high, as a lot of parameters were required for simulation and detailed knowledge on how to model PV systems and their stringing configurations. PVsyst, Archelios and SAM will provide basic help for system sizing combinations and recommendations, whereas PV*SOL does not provide recommendations. PV*SOL requires the user to know the system sizing, inverters, string configurations, wiring and cabling of the systems. This lack of recommendations for system sizing and PV module and inverter compatibility can make it difficult for initial planning stages for a PV project when exact system sizing and wiring of the project are unknown.

EnergyPlus was the most difficult software tool to learn and required expertise knowledge on building energy simulations. EnergyPlus by default has no graphical userinterface, is not user-friendly and has a high learning curve. In order to perform a simple simulation in EnergyPlus, many parameters for the building must be defined, such as the type of construction materials, window, HVAC system, and so forth. This is not important when it comes to modeling of solar PV, however, the software needs this information to be defined in order to simulate. Third-party software tools such as DesignBuilder help to simplify the simulation process in EnergyPlus, by providing a functioning 3D environment for the modeling to take place, hence avoiding the need to manually define everything in text and IDF format. However, the simulation procedure even in DesignBuilder is complicated as there are still many variables that must be defined.

\subsubsection{Modeling Flexibility}

Modeling flexibility can be defined as the characteristic modifiability in which the software tool facilitates the incorporation of changes (Miguel et al., 2014). All the proprietary software tools PVsyst, HelioScope, PV*SOL and Archelios have little modeling flexibility. There is no way to introduce or modify any of the internal submodels or predefined functions. However, it is possible to add/create new components into their PV and inverter databases. 
EnergyPlus and SAM are both opensource software tools in which they offer much modeling flexibility. EnergyPlus is an open-source command line software tool that reads input and writes output to text files. It utilizes an IDF-Editor for creating input files with a text-based interface, and EP-Launch for managing input and output files to perform batch simulations. A lot of energy modeling software tools support data exchange with EnergyPlus as it is commonly used in the energy modeling industry. EnergyPlus is a collection of many program modules that work together to perform building energy simulation. EnergyPlus coding can be performed in the programming language of $\mathrm{C}++$, and all the source code is available to inspect and modify in the source code repository wiki.

Simulations in SAM can be customized using opensource coding. The SAM Simulation Core (SSC) software development kit (SDK) contains a package of tools which allows programmers to use SAM simulation modules (the SSC library) to simulate renewable energy projects. It is available in Windows, OS X, or Linux applications and compatible with $\mathrm{C} / \mathrm{C}++$, C\#, Java, Python, and MATLAB (Blair et al., 2014). One of the strengths of SAM is for running multiple simulations for advanced parametric analysis, the user can run batch simulations for example to determine the best results out of a set of 20 different tilt angles and to compare to other simulation parameters easily. SAM also has advanced simulation options that are able to perform parametric and sensitivity analyses, and statistical analysis capabilities for Monte Carlo simulation and weather variability (P50/P90) studies. It can also read input variables from Microsoft Excel. SAM is a versatile software tool for modeling and offers the usage of macros and scripting capabilities to expand upon the default functions for user customizability. 


\section{RESULTS \& DISCUSSIONS}

This chapter will discuss the results obtained from the simulations generated by the methodologies to estimate 3D solar energy potential. The chapter will present the results generated by the GIS modeling and mapping of the solar community, the benchmarking performance analysis of the six solar simulation software tools, a comparison of the physical functions and capabilities of the software tools, limitations of the research and a discussion on the recommended software tools for 3D solar community energy analysis.

\subsection{GIS Modeling of Solar Community Results}

This section will be providing a discussion about the results generated from the insolation maps for the cumulative case study area of the solar community (not on the individual building level). The methodology as detailed in Section 4.1 was executed on two different 3D models of the case study area in southern Ontario for the: (i) original site plan; and (ii) alternative solar community design (developed by industry partner). The solar community designs were modelled on an hourly basis over a simulated year, and hourly maps were generated and stored in a geodatabase of over $32 \mathrm{~GB}$ in size. Insolation maps were also produced for both 3D models of the solar community on an annual and monthly basis. This was a very computational and time intensive process that generated over 4000 maps.

It was found that on an annual basis, the original site design and alternative design would receive approximately $874.9 \mathrm{kWh} / \mathrm{m}^{2}$ and $918.1 \mathrm{kWh} / \mathrm{m}^{2}$, respectively, of solar radiation. That is a $4.7 \%$ increase in the amount of annual average solar radiation received. Figure 31 illustrates the average monthly solar radiation that the case study area would receive. The alternative solar community design receives a significant increase in average solar radiation $\left(\mathrm{Wh} / \mathrm{m}^{2}\right)$ on a monthly basis, as compared to the original design. Figure 32 depicts the average monthly percentage increase that the alternative design receives. It receives a maximum increase of approximately $5.7 \%$ in the months of February, March, September and October. 


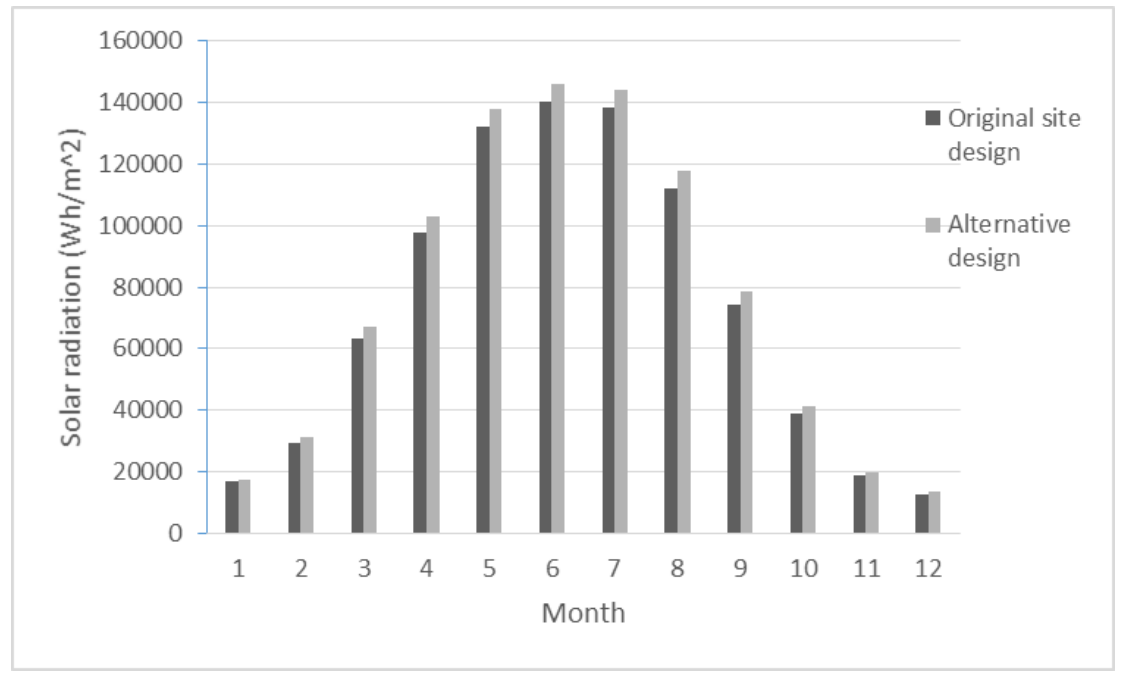

Figure 31. Monthly average hourly solar radiation of case study location in $\mathrm{Wh} / \mathrm{m}^{2}$.

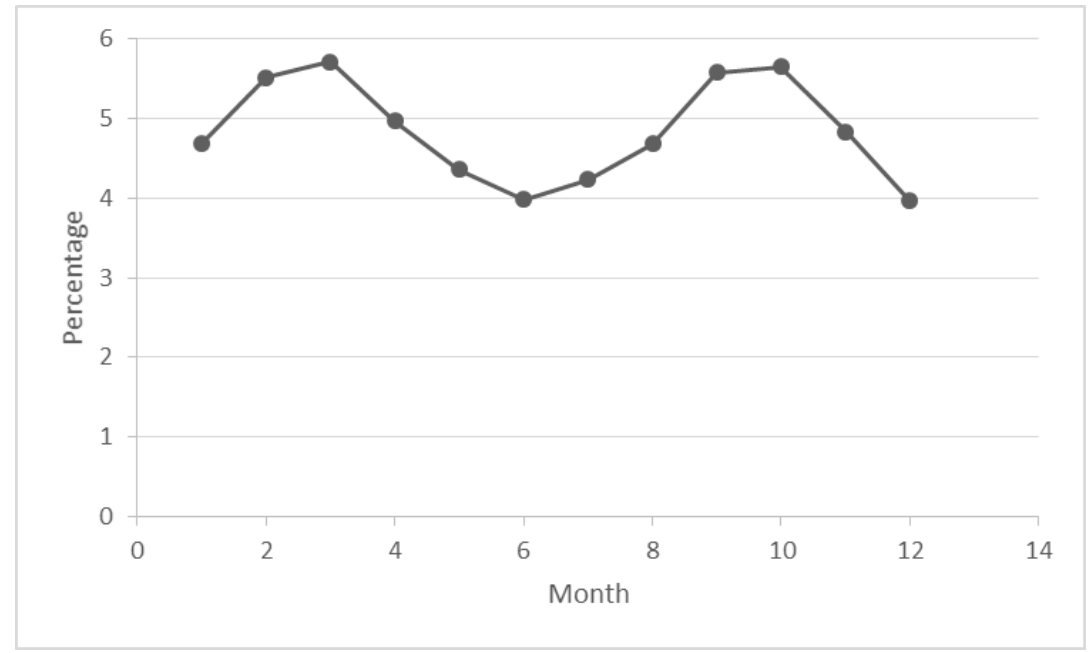

Figure 32. Percentage increase in average monthly solar radiation received for the alternative solar community design.

The results from the insolation maps for the case study area were examined on an hourly basis for the winter and summer solstices. The winter solstice (December 21st) is the day of the year that receives the least amount of daylight hours and experiences the largest amount of shadows. Nine daylight hours were generated by the software tool and mapped from the analysis for this particular date. The average solar radiation values were extracted from the hourly maps and the results for the analysis are depicted in Figure 33. 


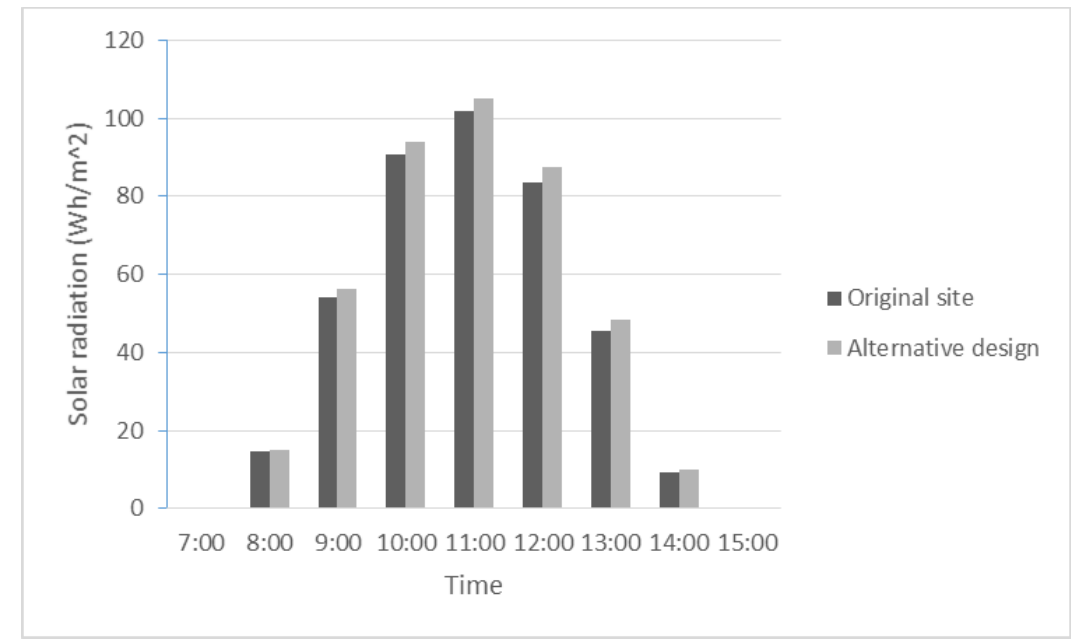

Figure 33. Average hourly solar radiation of case study location on the winter solstice.

These results indicate that the alternative design receives higher average hourly solar radiation in $\mathrm{Wh} / \mathrm{m}^{2}$, as compared to the original site plan design for every daylight hour. Notably, at $1 \mathrm{pm}$, the alternative design receives approximately 5\% more average solar radiation than the previous design, which is particularly useful for time-of-use design.

The summer solstice (June 21) is the day of the year that receives the most sunlight hours and experiences the least amount of shadows. There are approximately 16 hours of daylight on this date for the case study area location as a whole. The average solar radiation values were extracted from the maps and the results for the analysis are depicted in Figure 34.

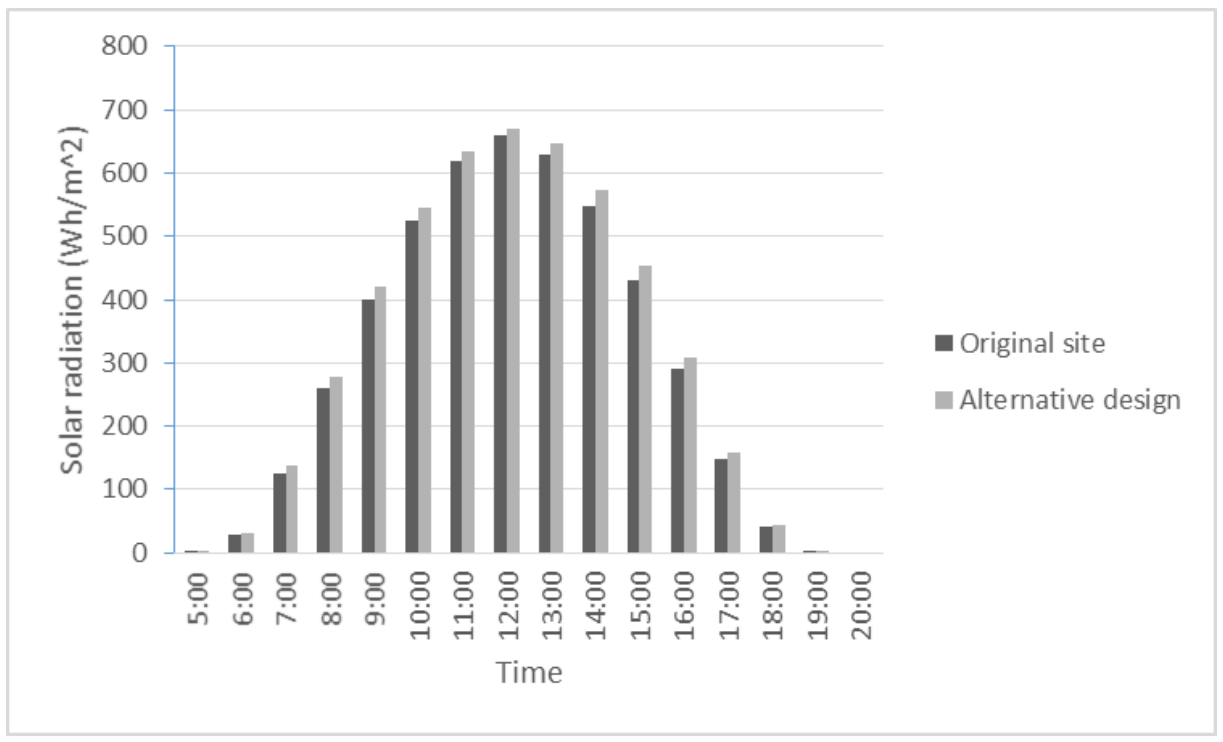

Figure 34. Average hourly solar radiation of case study location on the summer solstice. 
Figure 35 illustrates the percentage increase in the amount of average solar radiation received on an hourly basis for the winter and summer solstices. The alternative design receives more solar radiation on a consistent basis and receives up to $14 \%$ more solar radiation on the summer solstice around $6 \mathrm{am}$. Future work is recommended for the determination of the solar potential of each individual building unit (as opposed to the total site solar potential). However, this provides a good starting point for understanding how the cumulative solar radiation potential of the case study area and changes in building layout affect optimal solar community output.

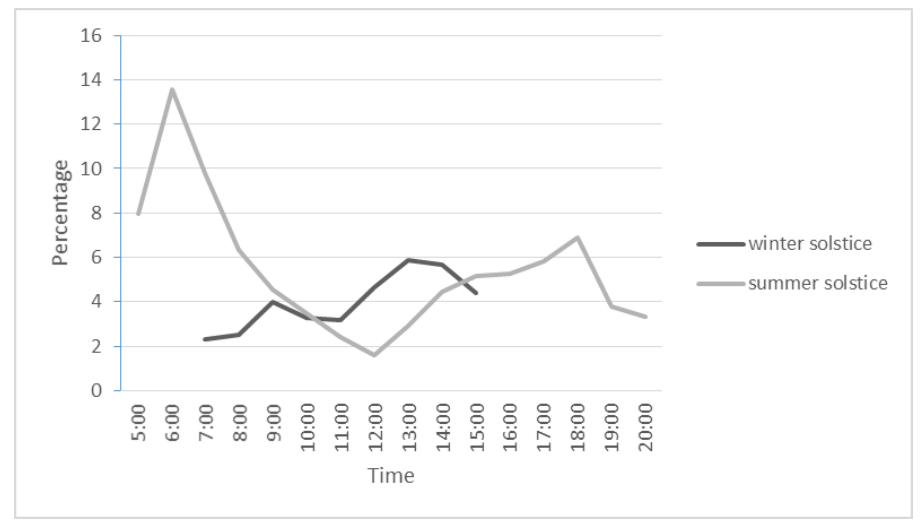

Figure 35. Percentage increase in average hourly solar radiation received for the winter and summer solstice.

\subsubsection{Limitations and Errors}

The limitations and sources of error for the developed GIS methodology can be mainly attributed to:

- Resolution of the sky size and input model used - this significantly effects the computational time required and level of accuracy of the analysis

- On-Screen digitization process of the building footprints - the on-screen digitization process in GIS is susceptible to human error, as it is a manual process for the tracing of the building footprint using a computer screen and mouse. It was performed at a $0.5 \mathrm{~m}$ resolution for building rooftops.

- Vertical facades - it was not possible to calculate the amount of irradiance received on the vertical facades of buildings using GIS due to the 2.5D nature of GIS 
- Extraction of data - the generated data had to be extracted for each hour of the year on each map, and an efficient iterative function is needed in order to speed the tedious process

- Solar PV electricity - there is no way to directly calculate the solar PV potential of a building without exporting the results to another software tool for analysis or the development of code

\subsection{Benchmarking of Solar Simulation Software Tool Results}

The benchmarking results of the software tools will be presented in two main sections, (1) results from the performance analysis, and (2) results from the comparison of the physical capabilities and functionality of the software tools.

\subsubsection{Performance Analysis Results}

Simulations were performed for the case study area using all 6 of the software tools, however, only results will be compared for 5 of the simulation software tools. The results that are not presented are the simulations that were performed using Archelios Pro, as unfortunately it was unable to simulate the exact configuration of the case study area. Hence, its results are omitted from the performance analysis results. A total of fourteen different simulation results are compared. Simulation variations performed in each tool are as indicated in Figure 36. 


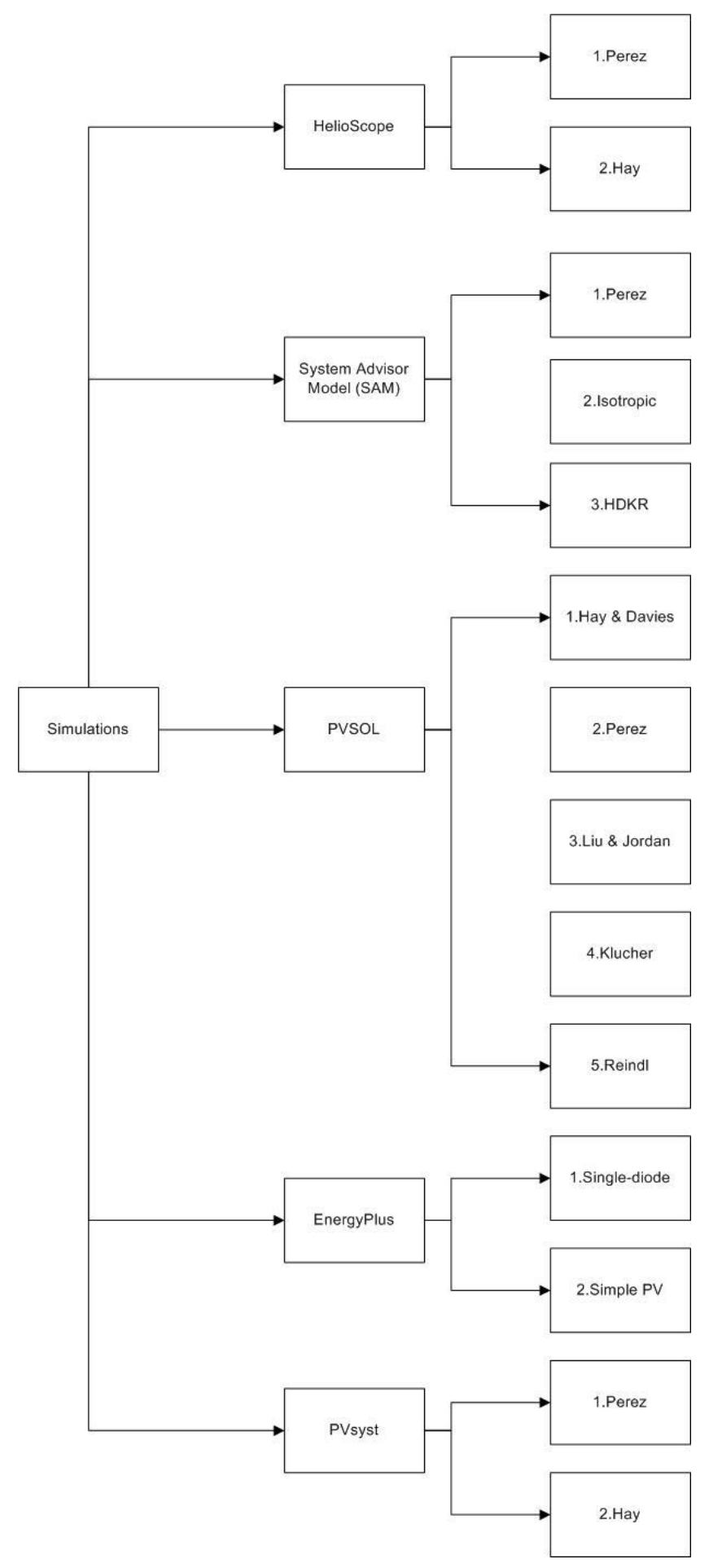

Figure 36. Simulations performed in each software tool. 
Note that for EnergyPlus, there was no option to change the transposition model. It only uses the Perez sky model, hence 2 different PV modeling options were evaluated instead.

\subsubsection{Annual PV Electricity Generation}

The measured annual PV generation for the case study house from January 1, 2018 to December 31, 2018 with downtime hours removed and nighttime inverter noise removed was found to be $9677.8 \mathrm{kWh}$. The annual results obtained from the simulations performed in the software tools were compared to the measured generation.

The annual percentage difference was calculated as:

$$
\text { Percentage Difference }(\%)=\frac{\text { modeled }- \text { measured }}{\text { measured }} * 100 \%
$$

This annual error of prediction quantified the overprediction or underprediction of the system. Figure 37 shows the annual prediction errors for the modeled to measured annual percentage differences as compared to annual measured generation.

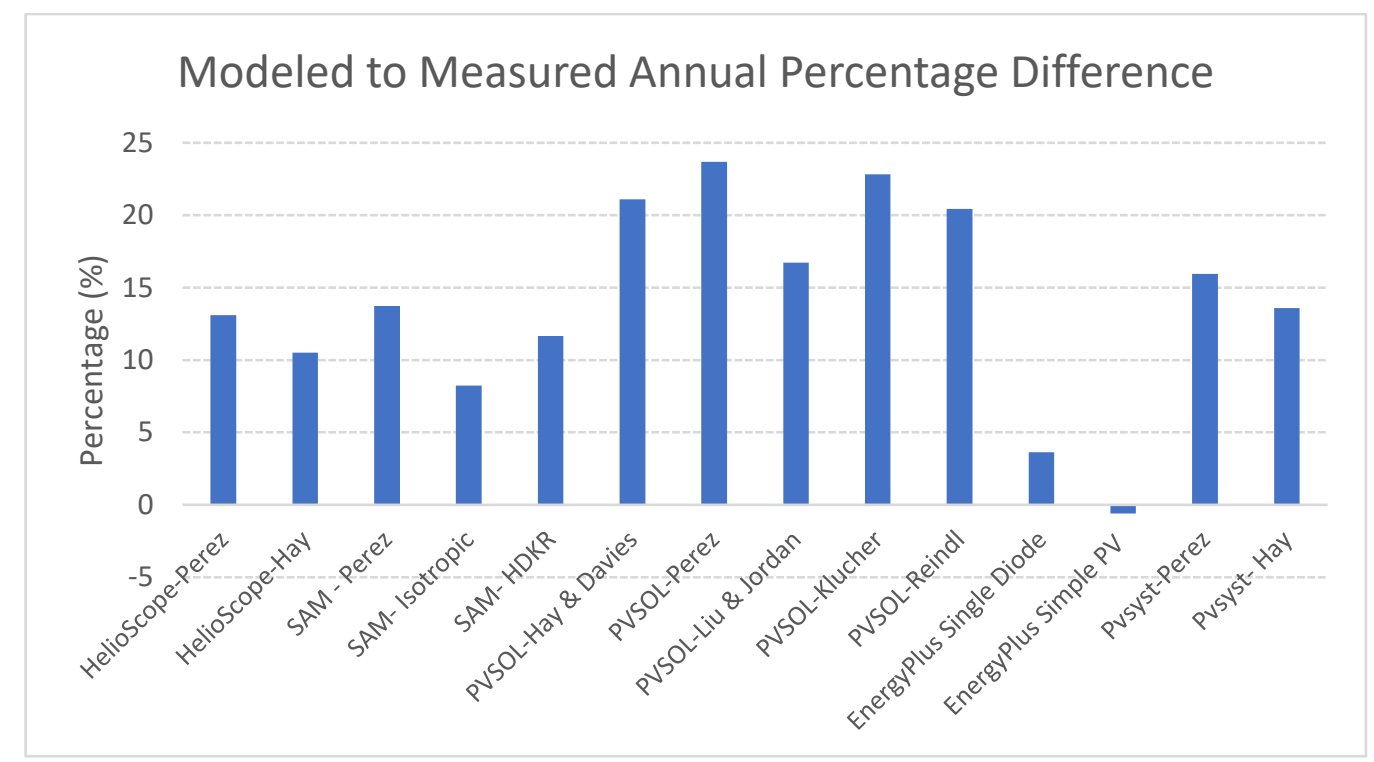

Figure 37. Annual modeled to measured percentage difference for HelioScope, SAM, PVSOL, EnergyPlus and PVsyst. 
The annual percentage prediction errors ranged from $-0.6 \%$ underestimation to $23.7 \%$ overestimation. The error range for the five software tools can be found in Table 7.

Table 7. Range of annual percentage error for HelioScope, SAM, PVSOL, EnergyPlus and PVsyst

\begin{tabular}{|c|c|}
\hline Software Tool & Error Range \\
\hline HelioScope & $10.5 \%-13.1 \%$ \\
\hline SAM & $8.2 \%-13.7 \%$ \\
\hline PVSOL & $16.7 \%-23.7 \%$ \\
\hline EnergyPlus & $-0.6 \%-3.6 \%$ \\
\hline PVsyst & $13.6 \%-15.9 \%$ \\
\hline
\end{tabular}

All the simulations overpredicted the system yield except for the EnergyPlus Simple PV model. EnergyPlus' Simple PV model was found to be the most accurate with an underestimation of annual yield by $-0.6 \%$. The next closest estimate was also performed by EnergyPlus using the Single-diode model with an overestimation of 3.6\%. PVSOL was found to have consistently produced the highest overestimations for the annual yield ranging from $16.7 \%$ to $23.7 \%$. This is attributable to the fact that PVSOL's default loss parameter settings are set lower than most of the software tools. For example, PVSOL's default annual losses due to soiling is set to $0 \%$, whereas in SAM the default value is $5 \%$.

Based upon the simulation results, the Perez model has consistently produced the highest annual yield estimates in HelioScope, SAM, PVSOL and PVsyst. The isotropic model in SAM and Liu \& Jordan isotropic model in PVSOL produced the most conservative estimates within the software tools.

\subsubsection{Monthly PV Electricity Generation}

The monthly generation profiles for the five software tools HelioScope, SAM, PVSOL, EnergyPlus and PVsyst were graphed against monthly measured generation in Figure 38 to Figure 42. 


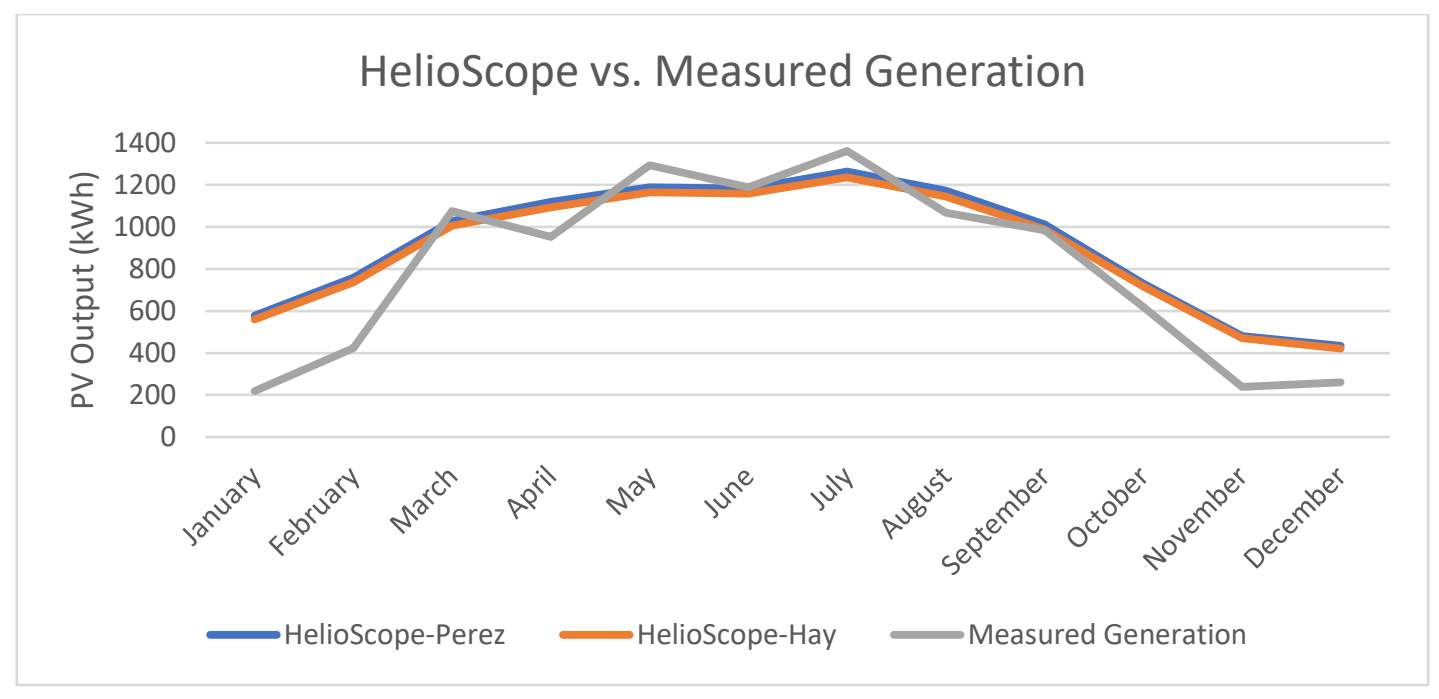

Figure 38. HelioScope monthly simulations compared to measured generation.

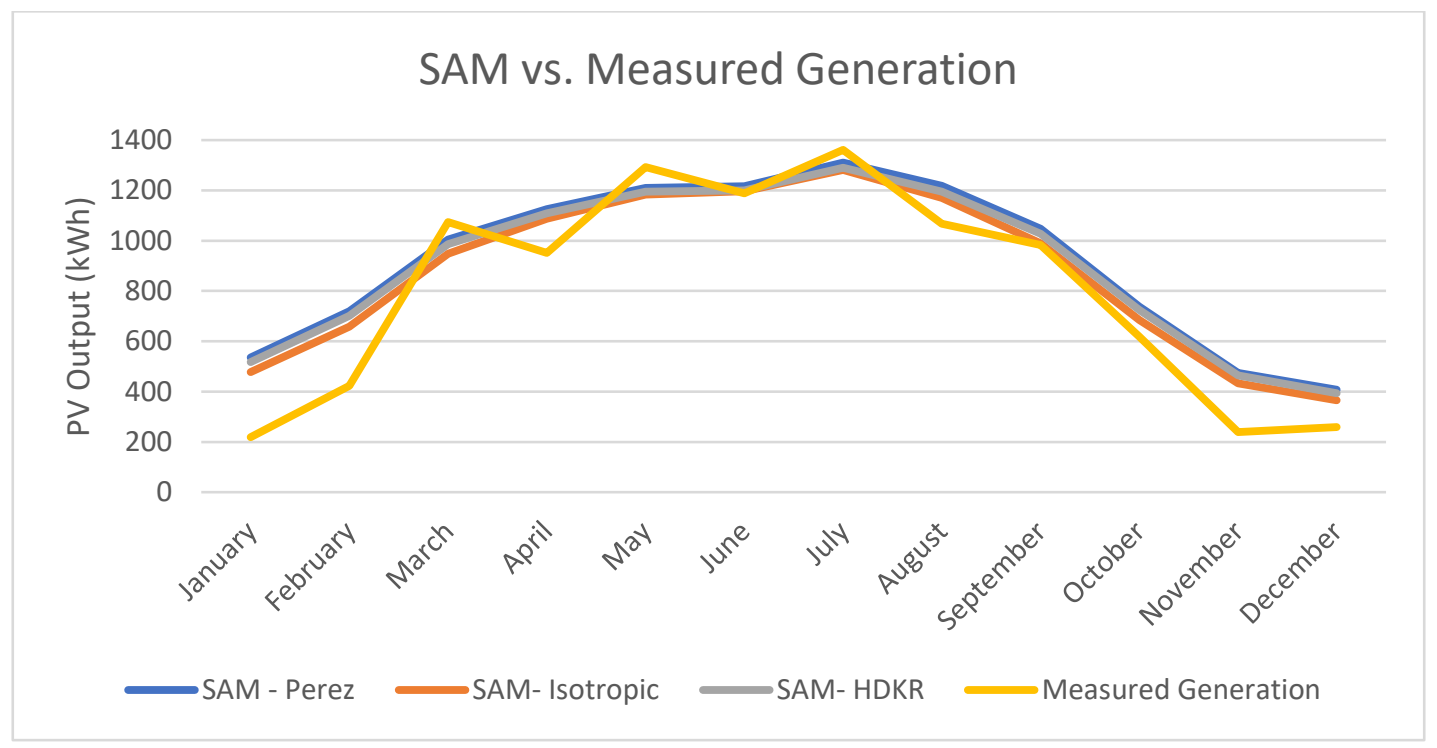

Figure 39. SAM monthly simulations compared to measured generation. 


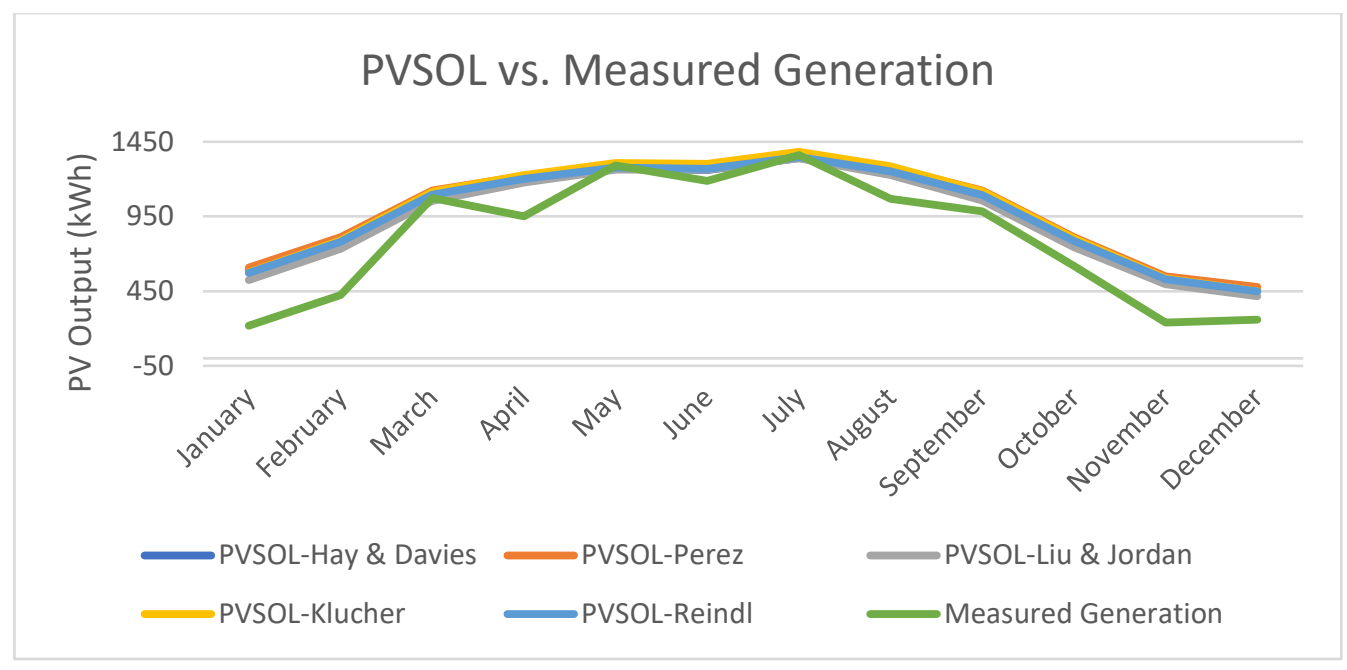

Figure 40. PVSOL monthly simulations compared to measured generation.

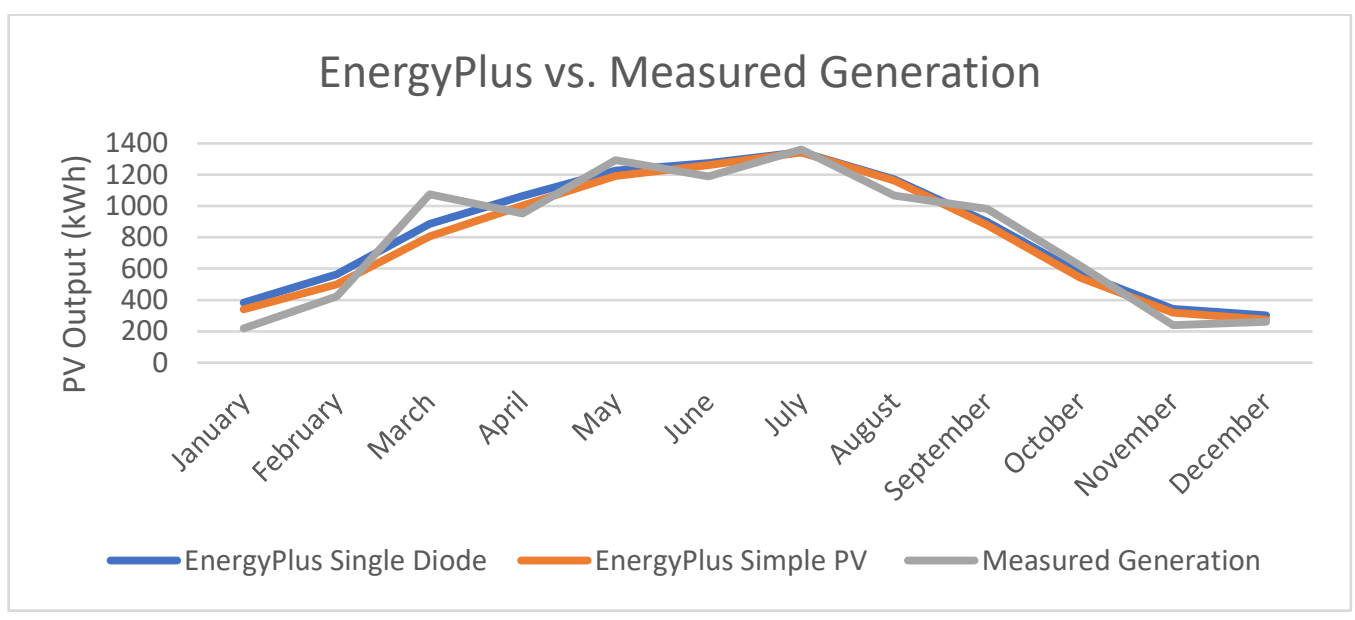

Figure 41. EnergyPlus monthly simulations compared to measured generation.

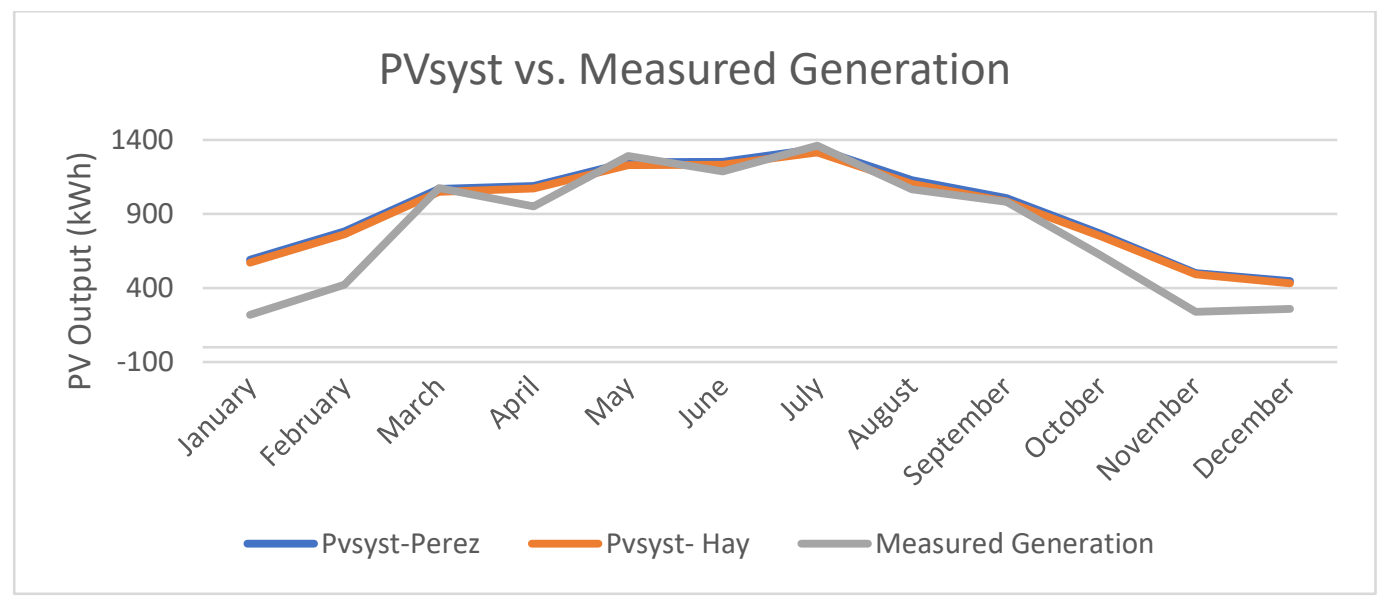

Figure 42. PVsyst monthly simulations compared to measured generation. 
It is evident from the graphs that the software tools all consistently overestimated generation in the winter months as compared to actual measured generation. This loss in generation is most likely due to snow cover in the winter months for the case study area.

Figure 43 shows a comparison of modeled to measured monthly results for the percentage difference in monthly prediction.

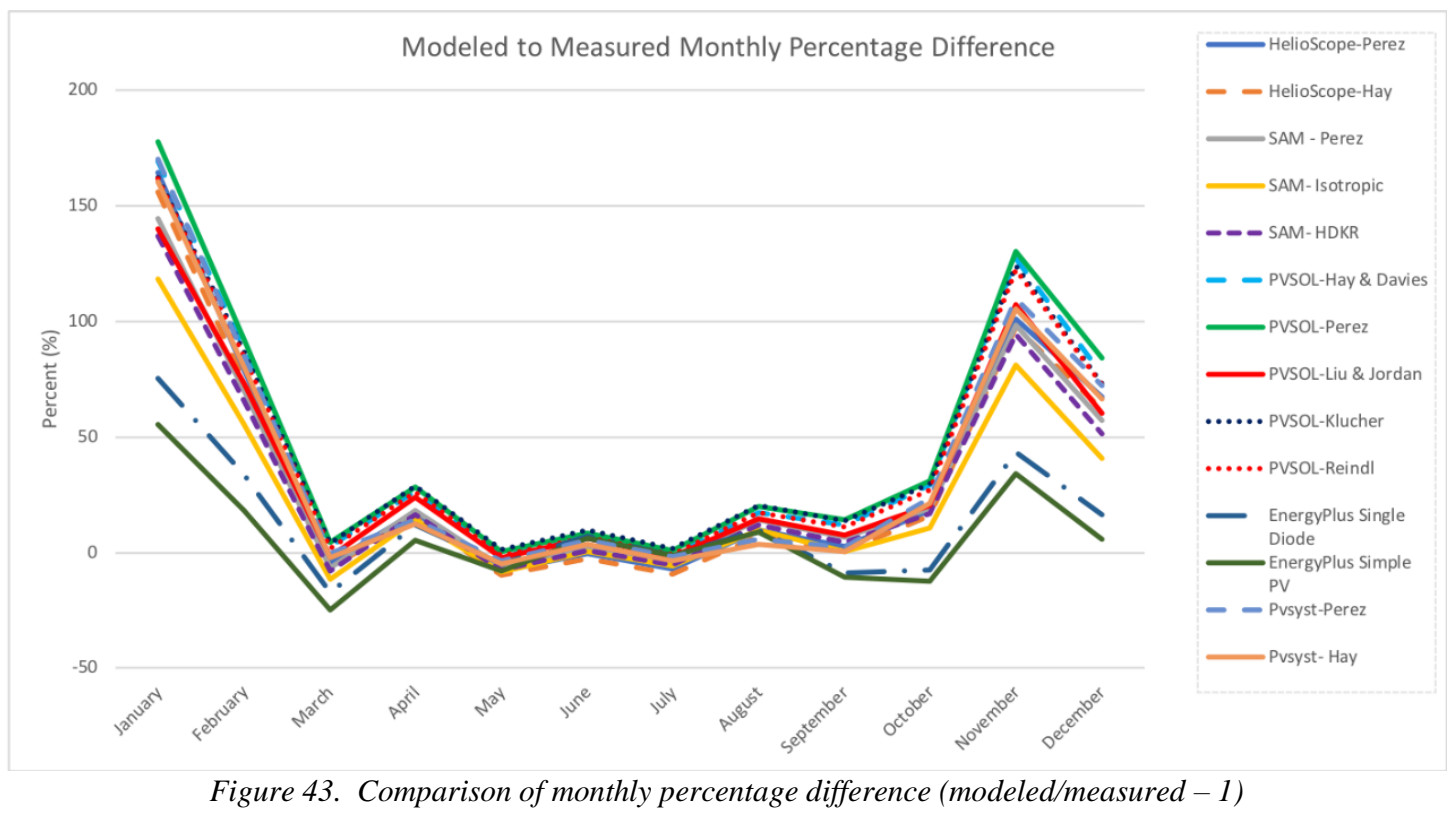

The overprediction in the winter months, especially from November to February is evident in all the software tools predictions. A summary of the percentage difference estimation range can be found in Table 8 showing which software tool the maximum and minimum predictions were made by. Upon analysis of Table 8 , it is evident that PVSOL is the most aggressive software tool for predictions on a monthly basis with the Perez and Klucher models. The highest overestimations can be seen in the winter months, with the most aggressive estimate of over 177.6\% produced in January by the PVSOL Perez model. A trend can be seen in which the EnergyPlus Simple PV model tends to underpredict consistently on a monthly basis except for in the months of May to August. The months in which the EnergyPlus Simple PV model produced the most conservative estimates also coincided with the months in which the PVSOL Perez model produced the most aggressive. Interestingly, when the EnergyPlus Simple PV 
model did not produce the minimum estimations, the minimum predictions were produced by the Hay model in both HelioScope and PVsyst for the summertime.

Table 8. Monthly percentage error range and software tool the maximum and minimum was predicted by.

\begin{tabular}{|c|c|c|c|}
\hline & \% Error Range & Min Predicted by & Max Predicted by \\
\hline January & $55.5-177.6 \%$ & EnergyPlus Simple PV & PVSOL Perez \\
\hline February & $18.0-92.0 \%$ & EnergyPlus Simple PV & PVSOL Perez \\
\hline March & $-25.0-4.6 \%$ & EnergyPlus Simple PV & PVSOL Perez \\
\hline April & $5.4-28.8 \%$ & EnergyPlus Simple PV & PVSOL Klucher \\
\hline May & $-9.9 \%-1.1 \%$ & HelioScope Hay & PVSOL Klucher \\
\hline June & $-2.5-9.6 \%$ & HelioScope Hay & PVSOL Klucher \\
\hline July & $-9.2-1.6 \%$ & HelioScope Hay & PVSOL Klucher \\
\hline August & $3.4-20.3 \%$ & PVsyst Hay & PVSOL Klucher \\
\hline September & $-10.8-14.2 \%$ & EnergyPlus Simple PV & PVSOL Perez \\
\hline October & $-12.4-31.0 \%$ & EnergyPlus Simple PV & PVSOL Perez \\
\hline November & $34.0-130.2 \%$ & EnergyPlus Simple PV & PVSOL Perez \\
\hline December & $5.9-84.1 \%$ & EnergyPlus Simple PV & PVSOL Perez \\
\hline
\end{tabular}

The percentage range of error for the summer months of May, June, and July had the least variability and were the closest to actual generation production. The minimum predictions which all underpredicted generation from -9.9 to $-2.5 \%$ for these months were produced by the HelioScope Hay model. The maximum predictions which overestimated from $1.1-9.6 \%$ were produced by the PVSOL Klucher model All the simulations produced consistent estimations within a $10 \%$ range of one another for these months.

\subsubsection{Average Monthly Hourly PV Generation}

The following twelve graphs depicted in Figure 44 to Figure 55 are comparisons of the performance of the different software tools used to predict solar PV generation. The graphs show the monthly average hourly generation profiles of the software tools, as compared to actual measured generation and GHI (from both measured and CWEC). Note that the measured GHI values were not available for the months of January and December 2018. It can be seen that the 
calculated generation for all the software tools followed the same pattern depicted on the graphs, although the magnitudes are different. The software tool that varied the most from the rest were the results generated by EnergyPlus.

Upon review of the twelve graphs it can be seen that the actual PV generation of the house (indicated by the blue line) does not closely follow any of the software simulated PV values. However, it is evident that the simulated values do follow the trend from the CWEC GHI weather data (red line with square markers) that was fed into the programs for simulation. For the months where measured GHI data was available, from February (Figure 45) to November (Figure 54), it is evident that the measured generation followed the pattern of the measured on-site GHI (red line with round markers). Hence, this shows that GHI is the most dominate factor affecting the PV output. For future research, it would be interesting to obtain measured data for the diffuse and direct components of solar radiation as well, to see which of the components would have a more significant effect on the accuracy of the simulated values.

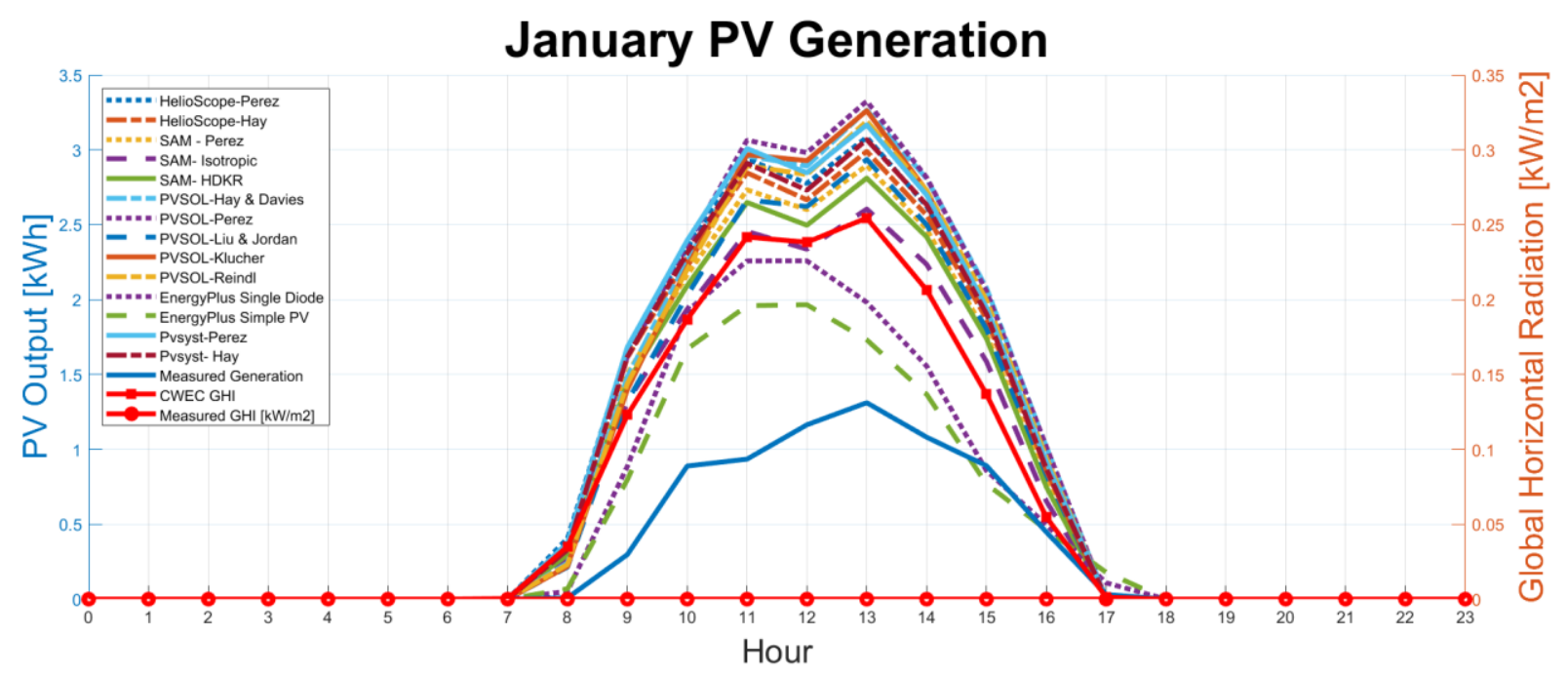

Figure 44. Average monthly hourly PV generation for January. 


\section{February PV Generation}

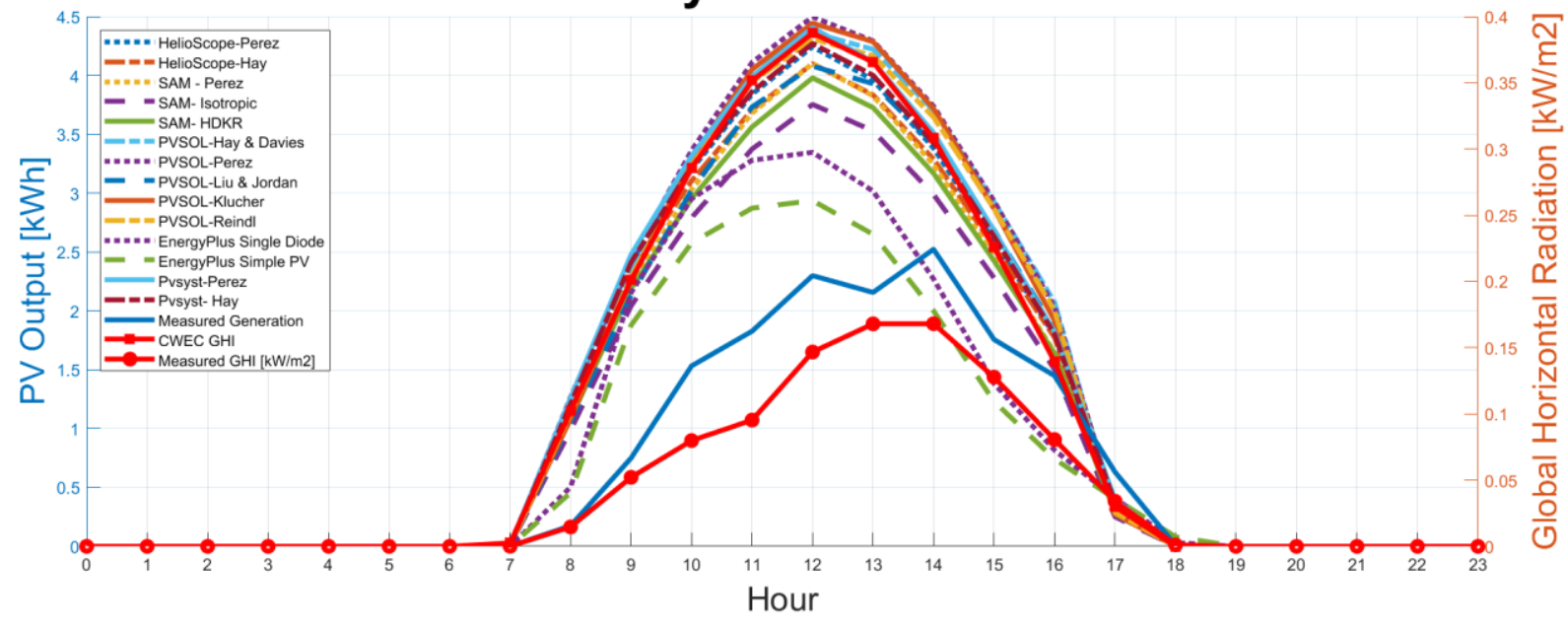

Figure 45. Average monthly hourly PV generation for February.

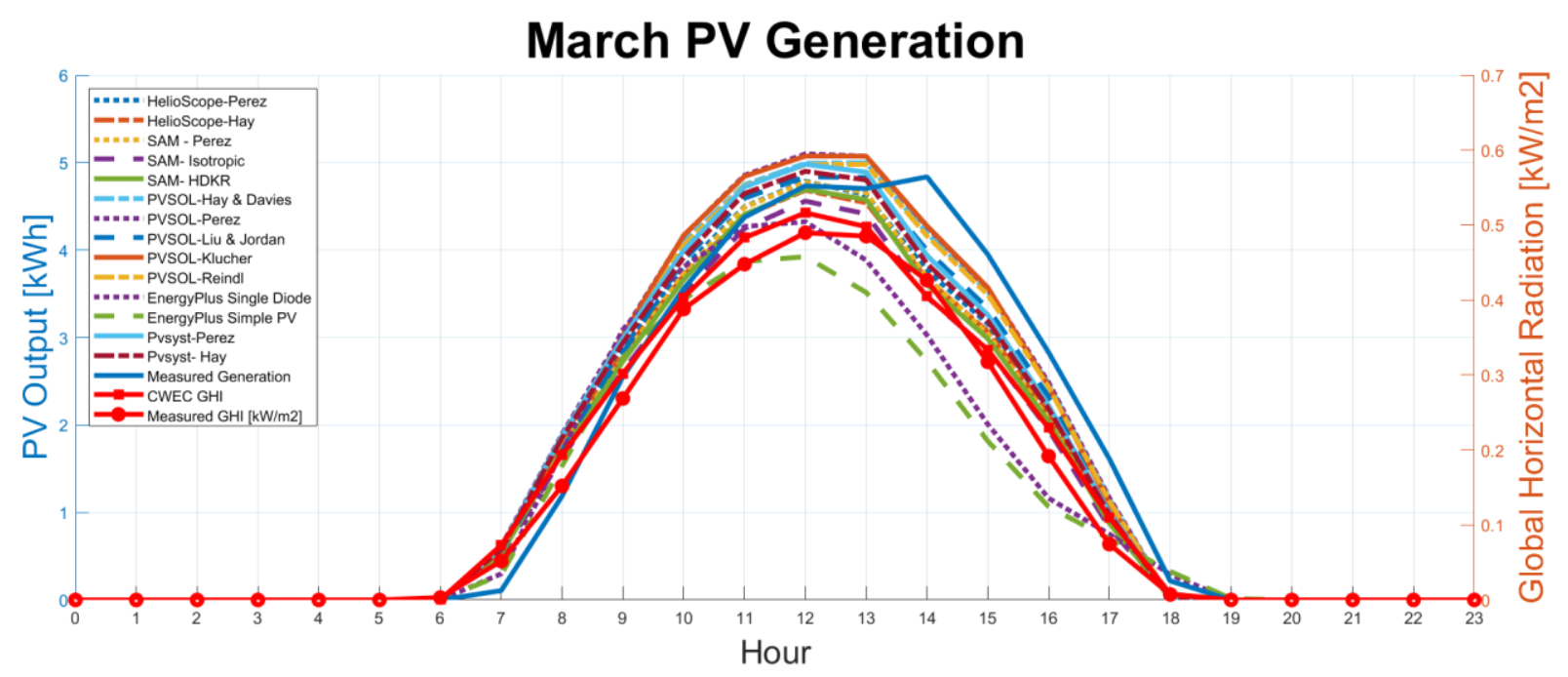

Figure 46. Average monthly hourly PV generation for March. 


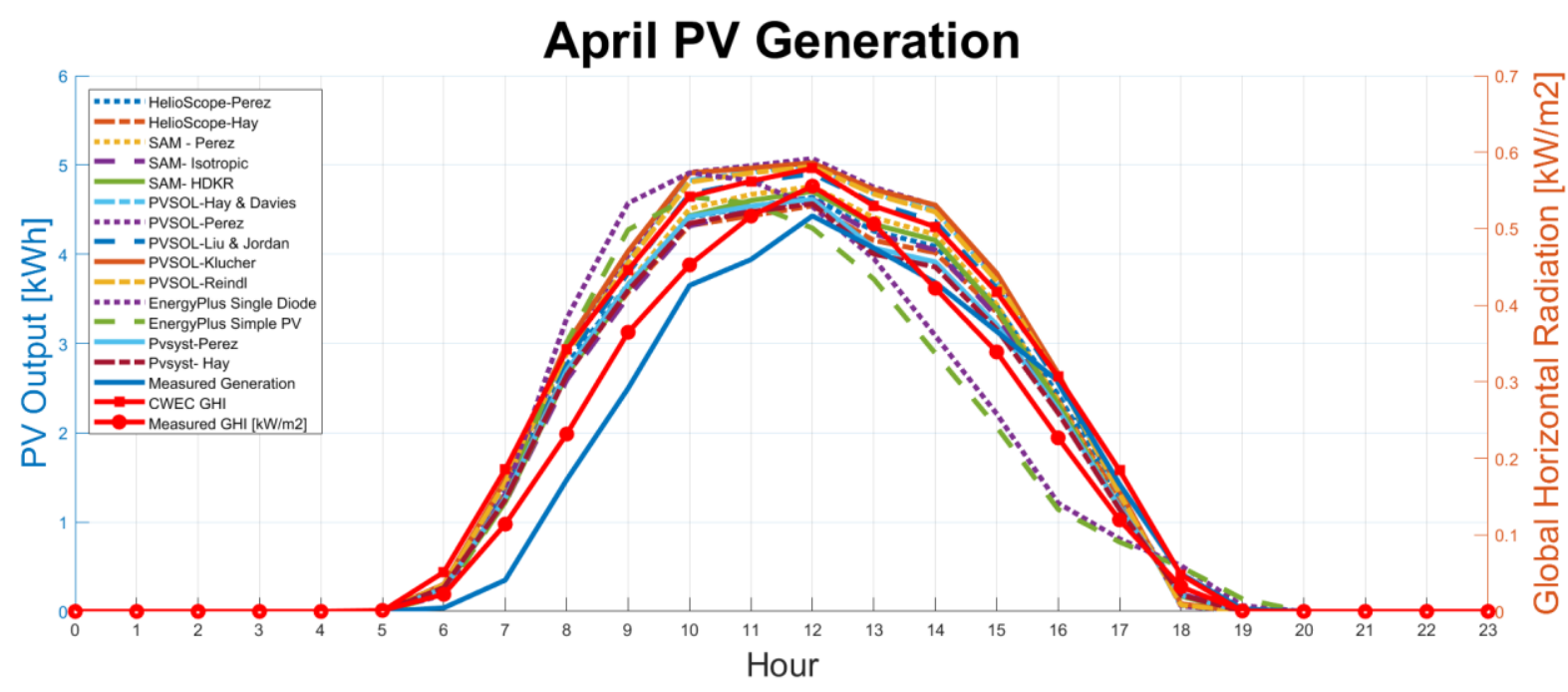

Figure 47. Average monthly hourly PV generation for April.

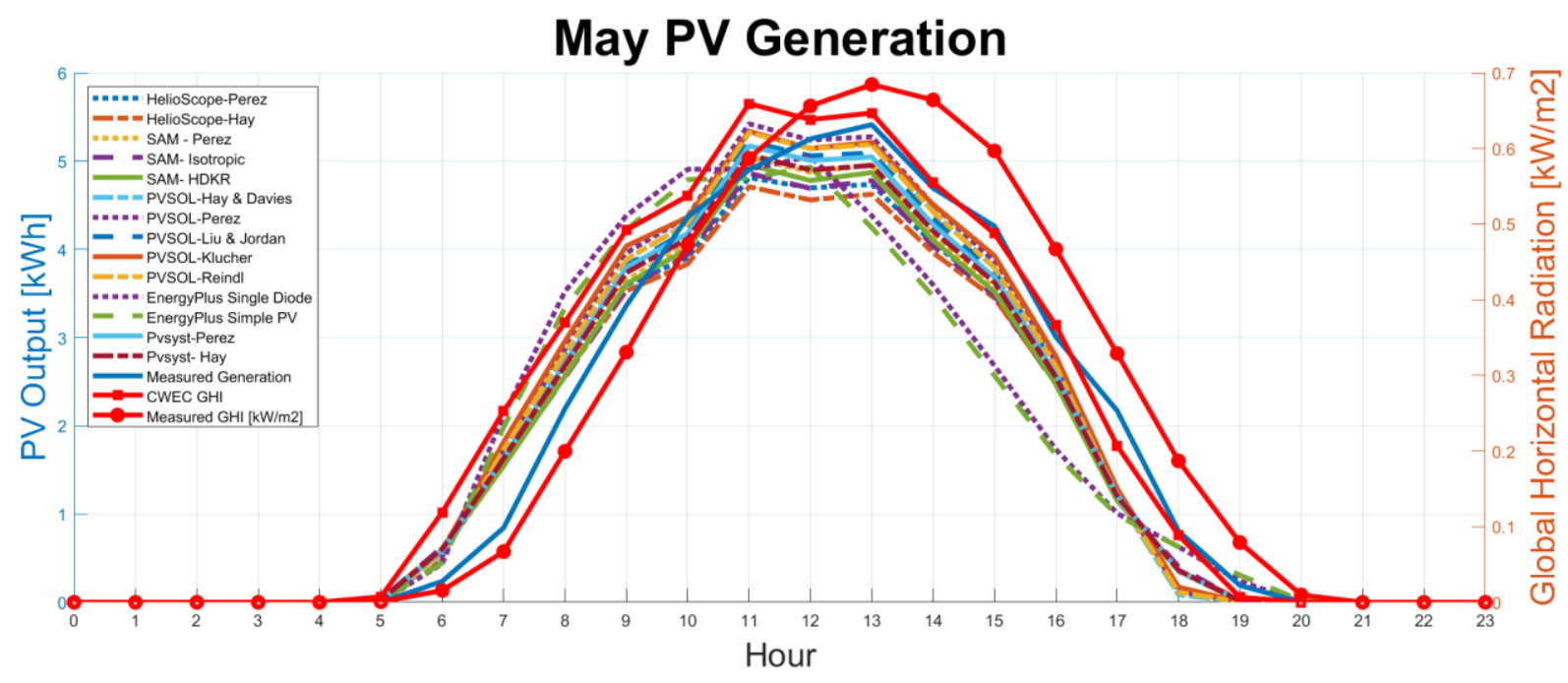

Figure 48. Average monthly hourly PV generation for May. 


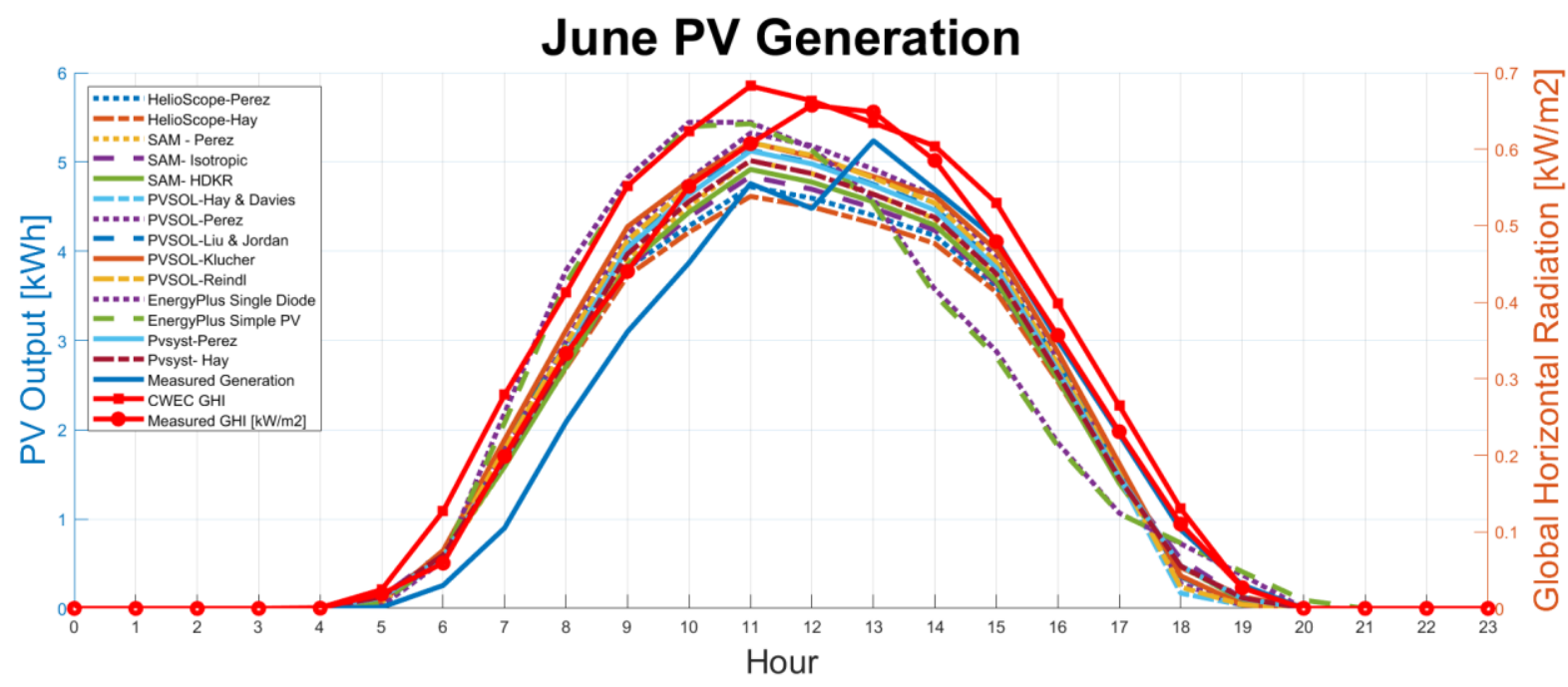

Figure 49. Average monthly hourly PV generation for June.

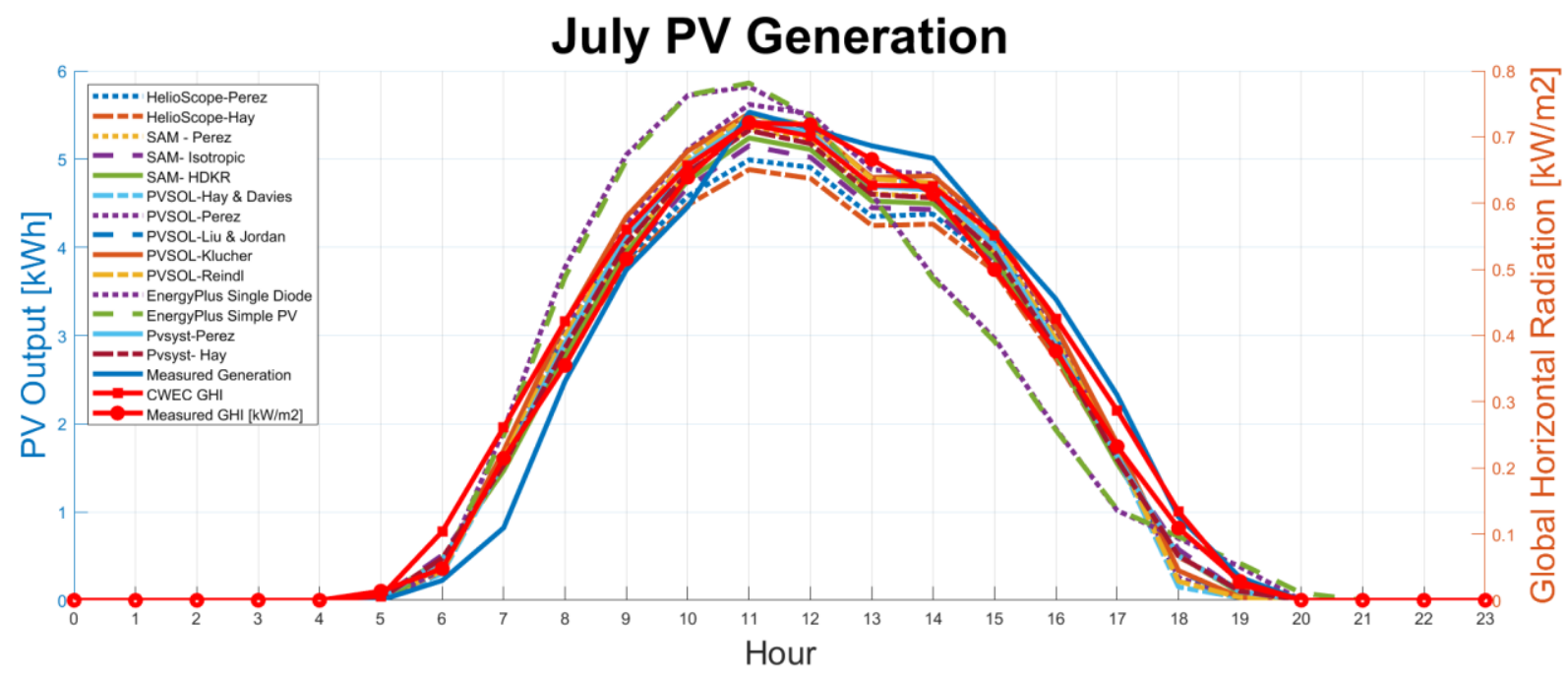

Figure 50. Average monthly hourly PV generation for July. 


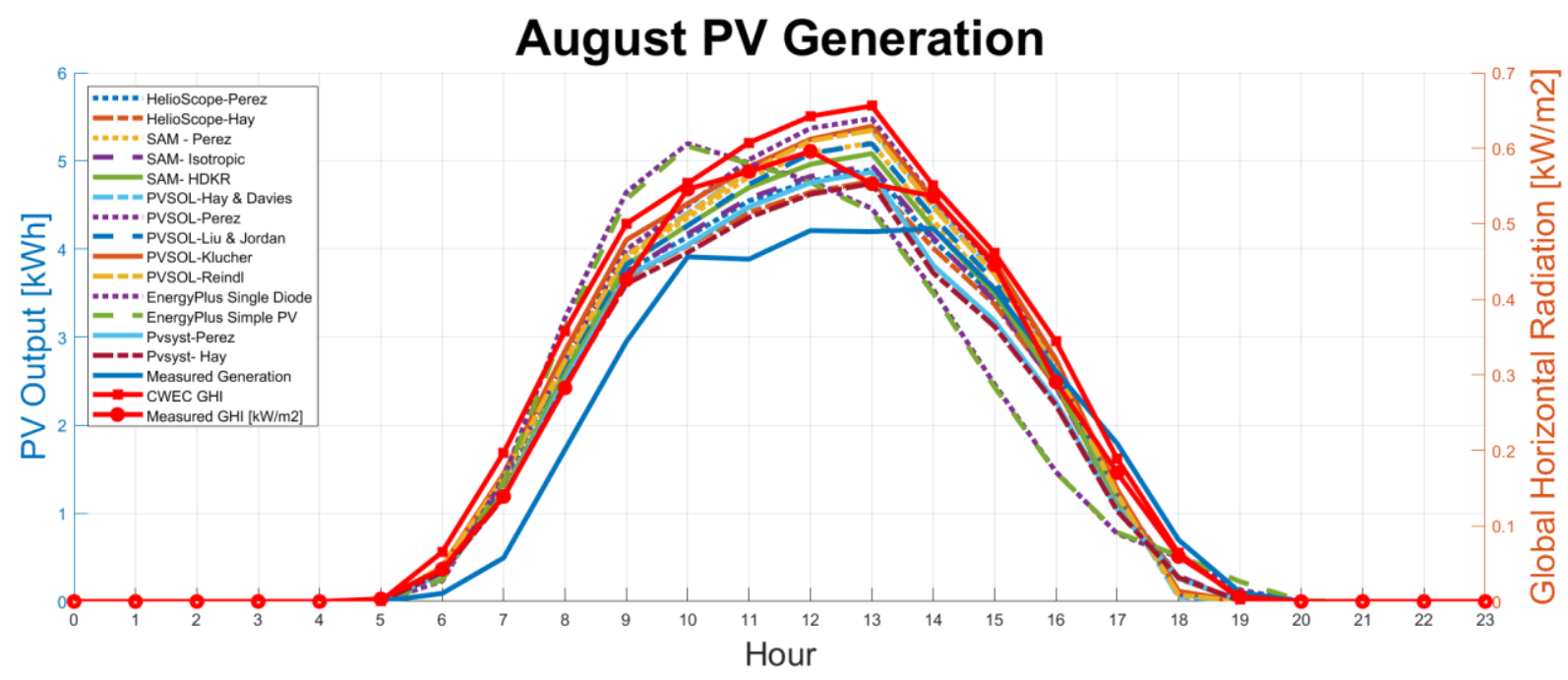

Figure 51. Average monthly hourly PV generation for August.

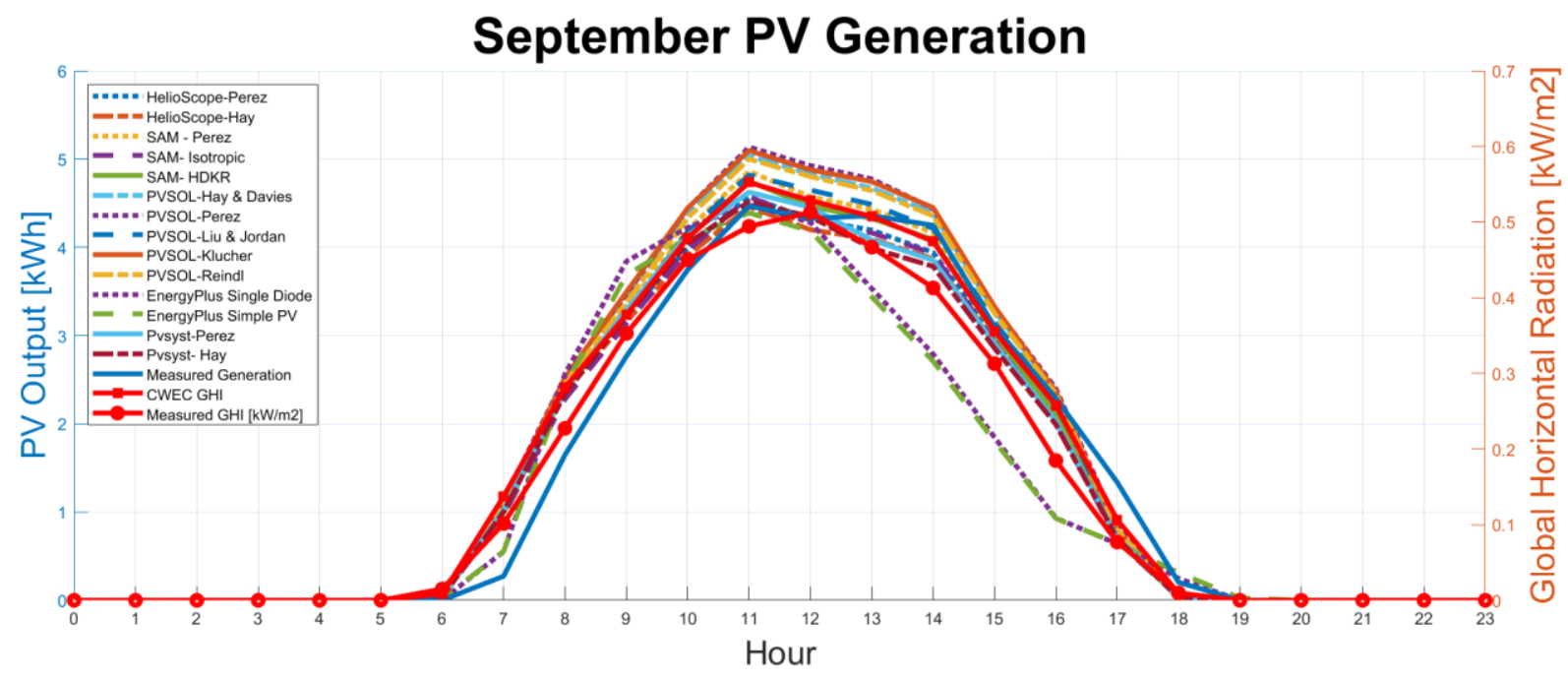

Figure 52. Average monthly hourly PV generation for September. 


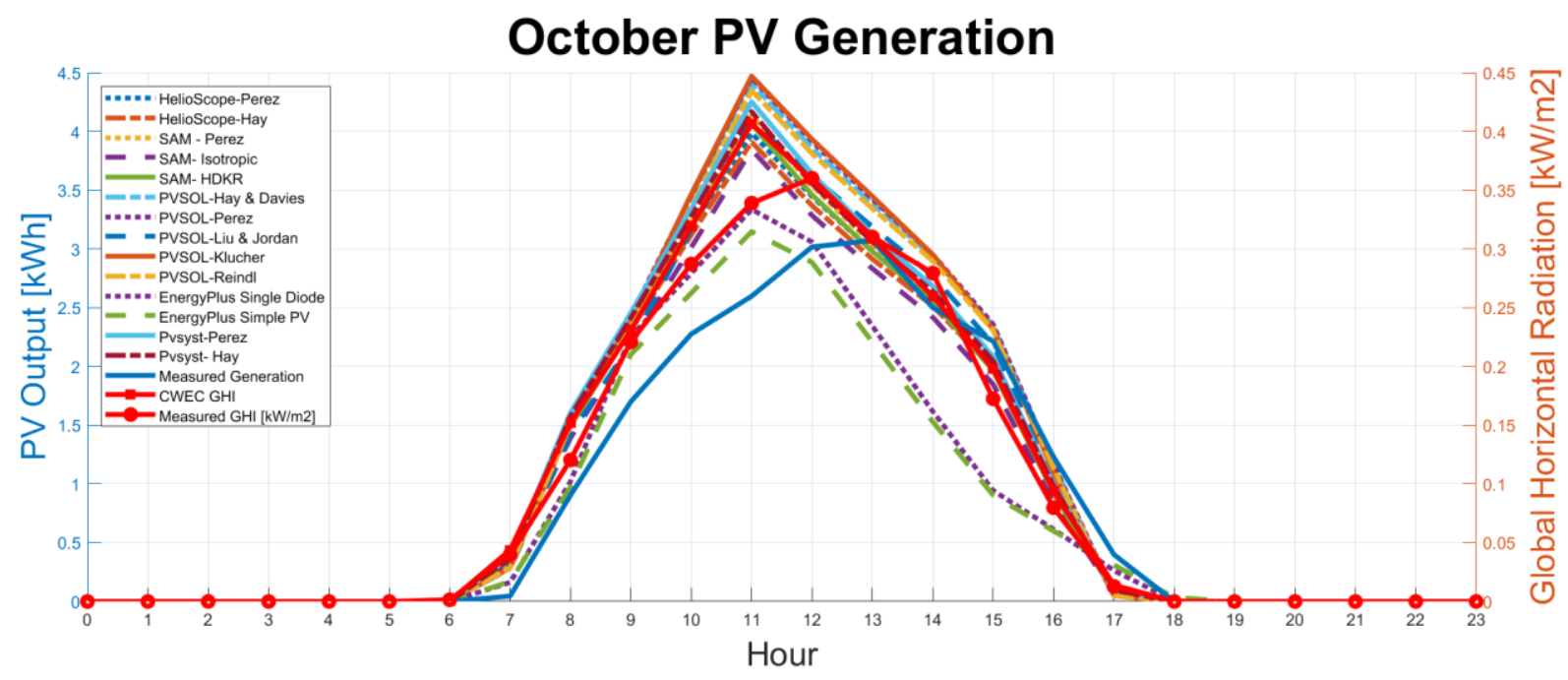

Figure 53. Average monthly hourly PV generation for October.

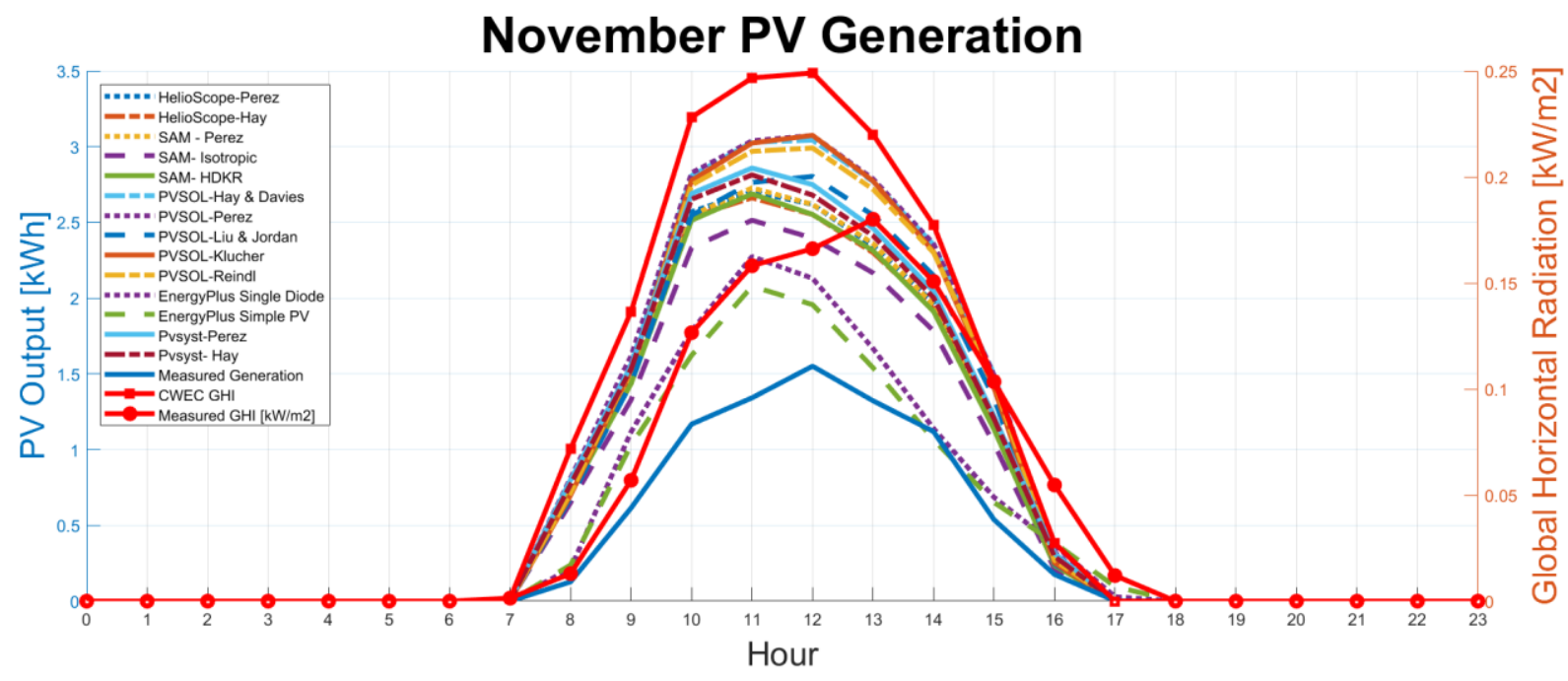

Figure 54. Average monthly hourly PV generation for November. 


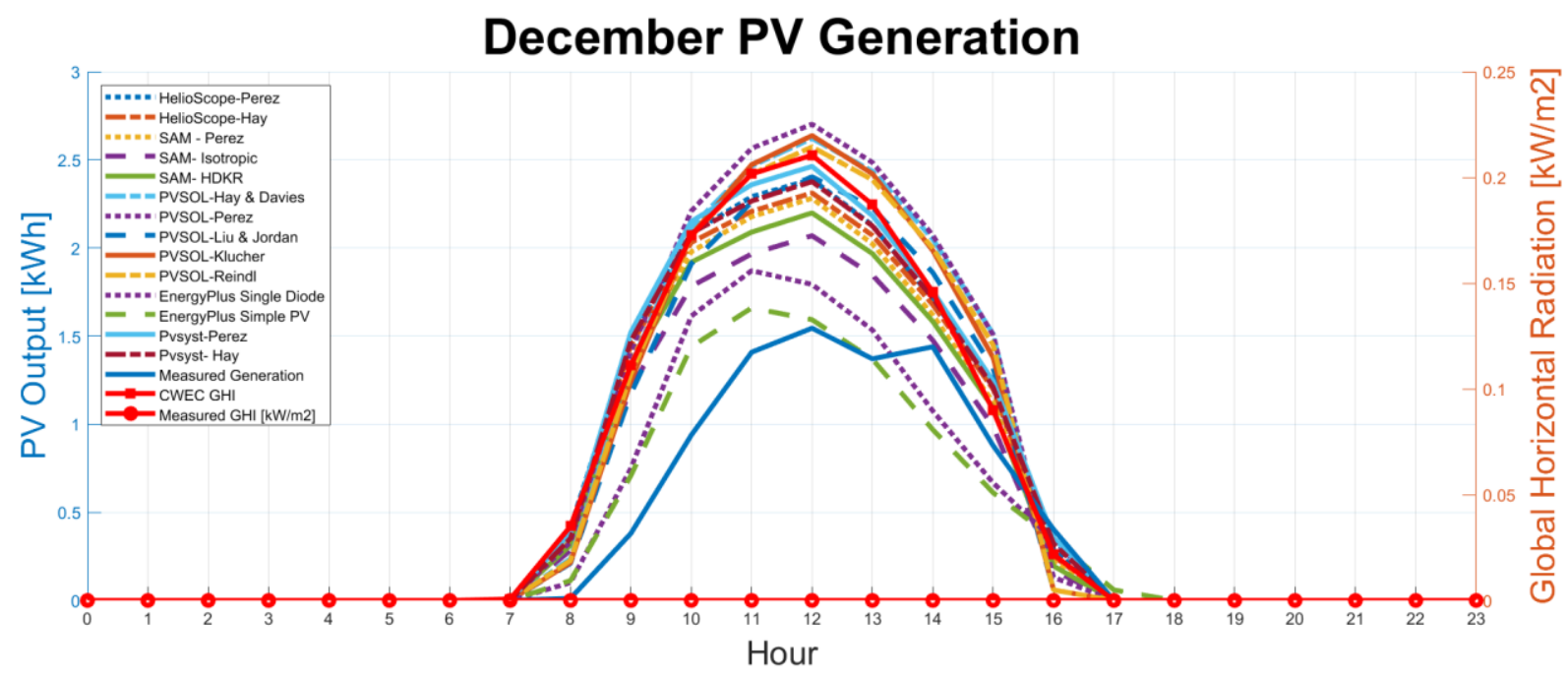

Figure 55. Average monthly hourly PV generation for December.

\subsubsection{Comparison of the Physical Functions and Capabilities of the Software Tools}

In order to help with the decision-making process of determining which software tools would be best suited for 3D solar community energy analysis, the physical functions and capabilities of the software tools needed to be examined. Based upon the determination of the physical functions and capabilities of the software tools that was presented in Section 4.2.3. Table 9 was created to summarize and compare HelioScope, PVsyst, PV*SOL, Archelios, EnergyPlus and SAM.

Table 9. Comparison of the physical functions and capabilities for HelioScope, PVsyst, PV*SOL, Archelios, EnergyPlus and SAM

\begin{tabular}{|l|l|l|l|l|l|l|l|}
\hline & & HelioScope & PVsyst & PV*SOL & Archelios & EnergyPlus & SAM \\
\hline 1 & Developer & $\begin{array}{l}\text { Folsom } \\
\text { Labs }\end{array}$ & $\begin{array}{l}\text { University } \\
\text { of Geneva }\end{array}$ & $\begin{array}{l}\text { Valentin } \\
\text { Software }\end{array}$ & $\begin{array}{l}\text { Trace } \\
\text { Software }\end{array}$ & NREL & NREL \\
\cline { 2 - 8 } & $\begin{array}{l}\text { Operating } \\
\text { System }\end{array}$ & $\begin{array}{l}\text { Internet } \\
\text { browsers }\end{array}$ & Windows & Windows & $\begin{array}{l}\text { Internet } \\
\text { browsers }\end{array}$ & $\begin{array}{l}\text { Windows, } \\
\text { Linux, and } \\
\text { Mac OS }\end{array}$ & $\begin{array}{l}\text { Windows, } \\
\text { Linux, and } \\
\text { Mac OS }\end{array}$ \\
\cline { 2 - 9 } & $\begin{array}{l}\text { Web-based } \\
\text { or } \\
\text { Application }\end{array}$ & Web & Application & Application & Web & Application & Application \\
\hline
\end{tabular}




\begin{tabular}{|c|c|c|c|c|c|c|c|}
\hline & Availability & Proprietary & Proprietary & Proprietary & Proprietary & Open source & Open source \\
\hline 2 & $\begin{array}{l}\text { Documentati } \\
\text { on \& } \\
\text { Maintenanc } \\
\text { e }\end{array}$ & $\begin{array}{l}\text { Detailed } \\
\text { documentati } \\
\text { on and } \\
\text { online help }\end{array}$ & $\begin{array}{l}\text { Detailed } \\
\text { documentati } \\
\text { on, forums } \\
\text { and updated } \\
\text { frequently }\end{array}$ & $\begin{array}{l}\text { Detailed } \\
\text { documentation } \\
\text {, online } \\
\text { support and } \\
\text { updated } \\
\text { frequently }\end{array}$ & $\begin{array}{l}\text { No detailed } \\
\text { documentati } \\
\text { on } \\
\text { available, } \\
\text { only an } \\
\text { FAQ, email } \\
\text { support }\end{array}$ & $\begin{array}{l}\text { Detailed } \\
\text { documentati } \\
\text { on, forums, } \\
\text { and updated } \\
\text { frequently }\end{array}$ & $\begin{array}{l}\text { Detailed } \\
\text { documentati } \\
\text { on, forums, } \\
\text { and updated } \\
\text { frequently }\end{array}$ \\
\hline 3 & $\begin{array}{l}\text { Weather } \\
\text { data }\end{array}$ & $\begin{array}{l}\text { EPW, } \\
\text { TMY2, } \\
\text { TMY3, and } \\
\text { Prospector } \\
\text { formats }\end{array}$ & $\begin{array}{l}\text { (3Tier) } \\
\text { Vaisala, } \\
\text { Canadian } \\
\text { EPW, } \\
\text { Explorador } \\
\text { Solar, } \\
\text { Meteonorm, } \\
\text { NREL } \\
\text { TMY2/TM } \\
\text { Y3, NREL } \\
\text { NSRDB, } \\
\text { PVGIS, } \\
\text { Satellight, } \\
\text { Soda- } \\
\text { Helioclim3, } \\
\text { Solar } \\
\text { Anywhere } \\
\text { (SUNY } \\
\text { model), } \\
\text { Solargis, } \\
\text { Solar } \\
\text { Prospector, } \\
\text { Vortex } \\
\text { Solar, } \\
\text { NASA SSE, } \\
\text { RETScreen } \\
\text { and WRDC } \\
\text { (World } \\
\text { Radiation } \\
\text { Data Center) }\end{array}$ & $\begin{array}{l}\text { DWD (*.xml), } \\
\text { Meteonorm } \\
(* . \text { dat), and } \\
\text { WBV (*.wbv) }\end{array}$ & $\begin{array}{l}\text { Archelios } \\
\text { meteo (csv), } \\
\text { PVGis (txt), } \\
\text { PVGis (web } \\
\text { service), } \\
\text { PVGis } \\
\text { (TMY), } \\
\text { NREL } \\
\text { (TMY3), } \\
\text { Helioclim } \\
\text { (TMY3), } \\
\text { Helioclim } \\
\text { PVsyst } \\
\text { (TMY), } \\
\text { Solargis } \\
\text { (TMY), and } \\
\text { Solargis } \\
\text { PVsyst } \\
\text { (TMY) }\end{array}$ & $\begin{array}{l}\text { Any formats } \\
\text { of TMY } \\
\text { weather data } \\
\text { formats such } \\
\text { as TMY2, } \\
\text { TMY3, and } \\
\text { EPW }\end{array}$ & $\begin{array}{l}\text { SAM CSV } \\
\text { format, } \\
\text { TMY3, } \\
\text { TMY2, and } \\
\text { EPW }\end{array}$ \\
\hline 4 & $\begin{array}{l}\text { Irradiance } \\
\text { Models }\end{array}$ & $\begin{array}{l}\text { Perez } \\
\text { (default) } \\
\text { and Hay } \\
\text { model }\end{array}$ & $\begin{array}{l}\text { Perez } \\
\text { (default) and } \\
\text { Hay model }\end{array}$ & $\begin{array}{l}\text { Hay \& Davies, } \\
\text { Liu \& Jordan, } \\
\text { Klucher, } \\
\text { Perez, and } \\
\text { Reindl model }\end{array}$ & Perez model & Perez model & $\begin{array}{l}\text { Isotropic, } \\
\text { HDKR, and } \\
\text { Perez } \\
\text { (default) } \\
\text { model }\end{array}$ \\
\hline \multirow[t]{2}{*}{5} & $\begin{array}{l}\text { Ability to } \\
\text { import 3D } \\
\text { model }\end{array}$ & No & Yes & Yes & Yes & $\begin{array}{l}\text { Yes } \\
\text { (depending } \\
\text { on third- } \\
\text { party } \\
\text { software } \\
\text { chosen) }\end{array}$ & No \\
\hline & $\begin{array}{l}\text { Supported } \\
\text { 3D data } \\
\text { formats }\end{array}$ & None & 3DS, DAE & $\begin{array}{l}\text { DirectX X } \\
(* . x), \\
\text { Wavefront } \\
\text { Object (*.obj), } \\
\text { Collada } \\
\text { (*.dae), } \\
\text { Blender 3D }\end{array}$ & $\begin{array}{l}\text { SketchUp } \\
\text { (.skp) }\end{array}$ & $\begin{array}{l}\text { CAD } \\
\text { formats vary } \\
\text { depending } \\
\text { on third- } \\
\text { party } \\
\text { software }\end{array}$ & $\begin{array}{l}. s 3 d \text { (SAM } \\
\text { native } \\
\text { format) }\end{array}$ \\
\hline
\end{tabular}




\begin{tabular}{|c|c|c|c|c|c|c|c|}
\hline & & & & $\begin{array}{l}\text { (*.blend), 3ds } \\
\text { MAX 3DS } \\
\text { (*.3ds), 3ds } \\
\text { MAX ASE } \\
\text { (*.ase), } \\
\text { LightWave } \\
\text { (*.lwo), } \\
\text { Stanford } \\
\text { Polygon } \\
\text { Library } \\
\text { (*.ply), } \\
\text { Stereolithogra } \\
\text { phy (*.stl), } \\
\text { and Milkshape } \\
\text { 3D (*.ms3d) }\end{array}$ & & & \\
\hline 6 & Shading & $\begin{array}{l}\text { Row-to-row } \\
\text { shading, } \\
\text { obstruction } \\
\text { shading } \\
\text { (keepouts), } \\
\text { and horizon } \\
\text { files (user } \\
\text { defined or } \\
\text { imported) }\end{array}$ & $\begin{array}{l}\text { Horizon } \\
\text { profile (user } \\
\text { defined or } \\
\text { imported), } \\
\text { near } \\
\text { shadings } \\
\text { modeled in } \\
\text { 3D } \\
\text { environment }\end{array}$ & $\begin{array}{l}\text { Horizon } \\
\text { profile (user } \\
\text { defined or } \\
\text { imported), 3D } \\
\text { visualization } \\
\text { mode for } \\
\text { shading } \\
\text { environment }\end{array}$ & $\begin{array}{l}\text { Horizon } \\
\text { profile (user } \\
\text { defined or } \\
\text { imported), } \\
\text { 3D shading } \\
\text { modeled in } \\
\text { SketchUp } \\
\text { environmen } \\
t\end{array}$ & $\begin{array}{l}\text { Requires } \\
\text { usage of } \\
\text { third-party } \\
\text { software for } \\
\text { 3D } \\
\text { modeling for } \\
\text { shading }\end{array}$ & $\begin{array}{l}\text { External } \\
\text { shading } \\
\text { modeled } \\
\text { through the } \\
\text { usage of } \\
\text { shade tables, } \\
\text { or the 3D } \\
\text { shade } \\
\text { calculator, } \\
\text { self-shading, } \\
\text { and snow } \\
\text { cover }\end{array}$ \\
\hline 7 & $\begin{array}{l}\text { Modeling } \\
\text { Size } \\
\text { Restrictions }\end{array}$ & $\begin{array}{l}\text { Recommen } \\
\text { ded } \\
\text { maximum } \\
\text { of } 5 \mathrm{MW}\end{array}$ & 8 subarrays & $\begin{array}{l}\text { In 3D mode - } \\
7500 \text { modules } \\
\text { for ground } \\
\text { mounted } \\
\text { systems, and } \\
10,000 \\
\text { modules for } \\
\text { roof systems }\end{array}$ & $\begin{array}{l}\text { No size } \\
\text { restriction } \\
\text { mentioned }\end{array}$ & $\begin{array}{l}\text { No size } \\
\text { restriction } \\
\text { mentioned }\end{array}$ & $\begin{array}{l}\text { Maximum } \\
\text { of four } \\
\text { subarrays } \\
\text { and eight } \\
\text { strings each } \\
\text { (32) }\end{array}$ \\
\hline 8 & PV Database & Yes & Yes & Yes & Yes & No & Yes \\
\hline & $\begin{array}{l}\text { Inverter } \\
\text { Database }\end{array}$ & Yes & Yes & Yes & Yes & No & Yes \\
\hline 9 & PV Model & $\begin{array}{l}\text { Single- } \\
\text { diode model }\end{array}$ & $\begin{array}{l}\text { Single-diode } \\
\text { model }\end{array}$ & $\begin{array}{l}\text { Single-diode } \\
\text { model }\end{array}$ & $\begin{array}{l}\text { Single- } \\
\text { diode model }\end{array}$ & $\begin{array}{l}\text { Simple PV } \\
\text { model, } \\
\text { single-diode } \\
\text { model }\end{array}$ & $\begin{array}{l}\text { Simple } \\
\text { efficiency } \\
\text { model, CEC } \\
\text { single-diode } \\
\text { model, } \\
\text { Sandia } \\
\text { model, and } \\
\text { IEC61853 } \\
\text { single-diode } \\
\text { model }\end{array}$ \\
\hline $\begin{array}{l}1 \\
\mathbf{0}\end{array}$ & $\begin{array}{l}\text { Inverter } \\
\text { Model }\end{array}$ & $\begin{array}{l}\text { Based upon } \\
\text { inverter } \\
\text { profile and } \\
\text { efficiency } \\
\text { curve }\end{array}$ & $\begin{array}{l}\text { Based upon } \\
\text { inverter } \\
\text { profile and } \\
\text { efficiency } \\
\text { curve }\end{array}$ & $\begin{array}{l}\text { Based upon } \\
\text { inverter profile } \\
\text { and efficiency } \\
\text { curve }\end{array}$ & $\begin{array}{l}\text { Based upon } \\
\text { inverter } \\
\text { profile and } \\
\text { efficiency } \\
\text { curve }\end{array}$ & $\begin{array}{l}\text { Simple } \\
\text { model, look } \\
\text { up table, or } \\
\text { function of } \\
\text { power }\end{array}$ & $\begin{array}{l}\text { Inverter } \\
\text { CEC } \\
\text { database or } \\
\text { datasheet, } \\
\text { inverter part } \\
\text { load curve } \\
\text { and inverter } \\
\text { CEC }\end{array}$ \\
\hline
\end{tabular}




\begin{tabular}{|c|c|c|c|c|c|c|c|}
\hline & & & & & & & $\begin{array}{l}\text { Coefficient } \\
\text { Generator }\end{array}$ \\
\hline 1 & $\begin{array}{l}\text { System } \\
\text { Losses (and } \\
\text { default } \\
\text { values) }\end{array}$ & $\begin{array}{l}\begin{array}{l}\text { Monthly } \\
\text { soiling } \\
\text { derate }=2 \%\end{array} \\
\text { AC system } \\
\text { derate = } \\
0.5 \%\end{array}$ & $\begin{array}{l}\text { DC circuit } \\
\text { ohmic losses } \\
=1.5 \% \\
\text { Module } \\
\text { efficiency } \\
\text { loss =0.5\% } \\
\text { LID = light } \\
\text { induced } \\
\text { degradation } \\
=2 \% \\
\text { Module } \\
\text { mismatch } \\
\text { losses- } \\
\text { power loss } \\
\text { at MPP = } \\
0 \% \\
\text { Module } \\
\text { mismatch } \\
\text { losses - loss } \\
\text { when } \\
\text { running at } \\
\text { fixed } \\
\text { voltage = } \\
0 \% \\
\text { Strings } \\
\text { voltage } \\
\text { mismatch- } \\
\text { power loss } \\
\text { at MPP = } \\
0 \% \\
\text { Yearly } \\
\text { soiling loss } \\
\text { factor - } 3 \% \\
\text { (can define } \\
\text { monthly } \\
\text { values as } \\
\text { well) } \\
\text { Unavailabili } \\
\text { ty of the } \\
\text { system- } \\
\text { unavailabilit } \\
\text { y time } \\
\text { fraction } 2 \% \text { for a } \\
\text { duration of } \\
7.30\end{array}$ & $\begin{array}{l}\text { Losses due to } \\
\text { deviation from } \\
\text { standard } \\
\text { spectrum = } \\
1 \% \\
\text { Power losses } \\
\text { resulting from } \\
\text { a drop in } \\
\text { voltage at the } \\
\text { bypass diodes } \\
=0.5 \% \\
\text { Power losses } \\
\text { resulting from } \\
\text { mismatching } \\
\text { or reduced } \\
\text { yield = 2\% } \\
\text { Ground } \\
\text { Reflection } \\
\text { (albedo) }= \\
20 \% \text { (annual } \\
\text { or monthly) } \\
\text { Losses due to } \\
\text { soiling = } 0 \% \\
\text { (annual or } \\
\text { monthly) }\end{array}$ & $\begin{array}{l}\begin{array}{l}\text { Monthly } \\
\text { albedo } \\
\text { values = } \\
20 \%\end{array} \\
\text { Losses in } \\
\text { DC wires - } \\
\text { STC = 1\% } \\
\text { Losses in } \\
\text { AC wires - } \\
\text { STC = 1\% } \\
\text { Module dirt } \\
\text { losses = 2\% } \\
\text { Module } \\
\text { aging = } \\
0.5 \% / y e a r \\
\text { LID = 0\% } \\
\text { Mismatch = } \\
2 \%\end{array}$ & $\mathrm{n} / \mathrm{a}$ & $\begin{array}{l}\text { Soiling loss } \\
\text { annual = 5\% } \\
\text { AC wiring = } \\
1 \% \\
\text { Transformer } \\
\text { losses = 0\% } \\
\text { Transmissio } \\
\text { n losses = } \\
0 \% \\
\text { Monthly } \\
\text { albedo } \\
\text { default = } \\
20 \% \\
\text { Curtailment } \\
\text { and } \\
\text { availability } \\
=0 \% \\
\text { Module } \\
\text { mismatch = } \\
0-2 \% \\
\text { Diodes and } \\
\text { Connections } \\
=0.5 \% \\
\text { DC wiring = } \\
2 \% \\
\text { Tracking } \\
\text { error = 0\% } \\
\text { Nameplate = } \\
0 \% \\
\text { DC power } \\
\text { optimizer } \\
\text { loss = 0-1\% }\end{array}$ \\
\hline
\end{tabular}




\begin{tabular}{|c|c|c|c|c|c|c|c|}
\hline & & & $\begin{array}{l}\text { days/year, } \\
\text { for } 3 \text { periods } \\
\text { Albedo } \\
\text { (monthly) = } \\
20 \%\end{array}$ & & & & \\
\hline $\begin{array}{l}1 \\
2\end{array}$ & $\begin{array}{l}\text { Cost of } \\
\text { Software } \\
\text { Tool }\end{array}$ & $\begin{array}{l}\text { \$95 } \\
\text { USD/month } \\
\text { Or \$950 } \\
\text { USD/year } \\
\text { Academic } \\
\text { and/or } \\
\text { student } \\
\text { pricing } \\
\text { available } \\
\text { upon } \\
\text { request }\end{array}$ & $\begin{array}{l}\text { CHF } \\
\text { 1300/year } \\
\text { Academic } \\
\text { and/or } \\
\text { student } \\
\text { pricing } \\
\text { available } \\
\text { upon request }\end{array}$ & $\begin{array}{l}€ 1295 / \text { year } \\
\text { Academic } \\
\text { and/or student } \\
\text { pricing } \\
\text { available upon } \\
\text { request }\end{array}$ & $\begin{array}{l}\text { €990/year } \\
\text { Academic } \\
\text { and/or } \\
\text { student } \\
\text { pricing } \\
\text { available } \\
\text { upon } \\
\text { request }\end{array}$ & $\begin{array}{l}\begin{array}{l}\text { Free of } \\
\text { charge }\end{array}\end{array}$ & $\begin{array}{l}\text { Free of } \\
\text { charge }\end{array}$ \\
\hline \multirow[t]{2}{*}{$\begin{array}{l}1 \\
3\end{array}$} & $\begin{array}{l}\text { Ability to } \\
\text { Export } \\
\text { Hourly CSV } \\
\text { data }\end{array}$ & Yes & Yes & Yes & Yes & Yes & Yes \\
\hline & $\begin{array}{l}\text { Creates a } \\
\text { Summary } \\
\text { Report of } \\
\text { Project PDF }\end{array}$ & Yes & Yes & Yes & Yes & No & Yes \\
\hline \multirow[t]{2}{*}{$\begin{array}{l}1 \\
4\end{array}$} & $\begin{array}{l}\text { User- } \\
\text { friendliness }\end{array}$ & $\begin{array}{l}\text { User- } \\
\text { friendly }\end{array}$ & Moderate & Moderate & $\begin{array}{l}\text { Not-user } \\
\text { friendly }\end{array}$ & $\begin{array}{l}\text { Not-user- } \\
\text { friendly }\end{array}$ & Moderate \\
\hline & $\begin{array}{l}\text { Level of } \\
\text { Expertise } \\
\text { Required }\end{array}$ & Moderate & High & High & High & High & High \\
\hline $\begin{array}{l}1 \\
5\end{array}$ & $\begin{array}{l}\text { Modeling } \\
\text { Flexibility }\end{array}$ & Not flexible & Not flexible & Not flexible & Not flexible & $\begin{array}{l}\text { Flexible - } \\
\text { Opensource } \\
\text { coding } \\
\text { available for } \\
\text { modification } \\
\mathrm{s}\end{array}$ & $\begin{array}{l}\text { Flexible - } \\
\text { Opensource } \\
\text { coding } \\
\text { available for } \\
\text { modification } \\
\mathrm{s}\end{array}$ \\
\hline
\end{tabular}

The information provided in Table 9, was created based upon the 15 functions and capabilities of the software tools. Based upon the review of the information presented, the software tool or tools that performed the best in each of the 15 categories were selected and presented in Table 10 . 
Table 10. Software tools recommended for best physical functions and capabilities

\begin{tabular}{|l|l|l|}
\hline & Software Tool(s) Recommended & Reason \\
\hline $\begin{array}{l}\text { 1.System Requirements and } \\
\text { availability }\end{array}$ & SAM and EnergyPlus & $\begin{array}{l}\text { Have the best capabilities as they } \\
\text { are available for free, open-source } \\
\text { and cross platform simulation tools }\end{array}$ \\
\hline 2. Documentation \& Maintenance & $\begin{array}{l}\text { HelioScope, PVsyst, PV*SOL, } \\
\text { EnergyPlus, and SAM }\end{array}$ & $\begin{array}{l}\text { All have very detailed } \\
\text { documentation and updated } \\
\text { frequently }\end{array}$ \\
\hline 3. Weather data & PVsyst & Most options available \\
\hline 4. Solar radiation models & PV*SOL & Most options available \\
\hline 5. 3D modeling capabilities & PV*SOL & $\begin{array}{l}\text { Most importation CAD formats } \\
\text { available }\end{array}$ \\
\hline 6. Shade modeling & $\begin{array}{l}\text { HelioScope, PVsyst, PV*SOL, } \\
\text { Archelios, and EnergyPlus }\end{array}$ & $\begin{array}{l}\text { Based upon detailed 3D drawing of } \\
\text { environment }\end{array}$ \\
\hline 7. Maximum modeling capabilities & Archelios and EnergyPlus & $\begin{array}{l}\text { No documented size restrictions on } \\
\text { modeling }\end{array}$ \\
\hline $\begin{array}{l}\text { 8. PV and Inverter Component } \\
\text { Database }\end{array}$ & PV*SOL & $\begin{array}{l}\text { Data is updated very frequently and } \\
\text { has large manufacturer database }\end{array}$ \\
\hline 9. PV Modeling & SAM & Most options available \\
\hline 10. Inverter Modeling & SAM & $\begin{array}{l}\text { Hast options available } \\
\text { loss most variables for system }\end{array}$ \\
\hline 11. Modeling of System Losses & PVsyst & Available for free \\
\hline 12. Cost of Software Tool & EnergyPlus and SAM & $\begin{array}{l}\text { Provides pdf reporting options and } \\
\text { detailed hourly results for various } \\
\text { parameters }\end{array}$ \\
\hline 13. Report and Analysis Ops & $\begin{array}{l}\text { HelioScope, PVsyst, PV*SOL, and } \\
\text { SAM }\end{array}$ & $\begin{array}{l}\text { Most user-friendly and least amount } \\
\text { of input parameters required }\end{array}$ \\
\hline $\begin{array}{l}\text { 14. User-friendliness \& Level of } \\
\text { Expertise }\end{array}$ & HelioScope & Programming options available \\
\hline 15. Modeling Flexibility & EnergyPlus and SAM & . \\
\hline
\end{tabular}

Based upon the recommendations of the software tools that performed best in each category as identified in Table 10, SAM was the overall software tool that performed the best for seven out of the fifteen functions, and Archelios the least with only two out of the fifteen evaluation criteria. A summary of the individual software tools findings are as follows:

HelioScope was recommended four times for its performance in:

- documentation and maintenance,

- shade modeling,

- report and analysis options

- user-friendliness and level of expertise.

PVsyst was recommended five times for its performance in:

- documentation and maintenance 
- weather data

- shade modeling

- modeling of system losses

- report and analysis options

PV*SOL was recommended a total of six times in the categories of:

- documentation and maintenance

- Solar radiation models

- 3D modeling capabilities

- Shade modeling

- PV and Inverter component Database

- Report and Analysis Options

Archelios was recommended two times for:

- Shade modeling

- Maximum modeling capabilities

EnergyPlus was recommended six times for:

- System Requirements and availability

- Documentation \& maintenance

- Shade modeling

- Maximum modeling capabilities

- Cost of software tool

- Modeling flexibility

SAM was recommended seven times for:

- System Requirements and availability

- Documentation \& Maintenance

- PV Modeling

- Inverter Modeling 
- Cost of software tool

- Report and Analysis Options

- Modeling Flexibility

It is evident that there is no one software tool that outperforms the rest in all of its physical functions and capabilities. The analysis of Table 10 helped to identify and highlight the strengths of the individual software tools. The main weaknesses of the software tools are highlighted as follows:

(1) HelioScope - The main deficiency of HelioScope is that there is no way to import 3D data into the software tool. Modeling has to be performed from scratch on the user interface, and the level of detail of the drawing capability is not as high as other CAD based software tools.

(2) PVsyst - PVsyst can be quite difficult to learn and requires a high level of expertise due to the complexity of its modeling parameters. User interface is not highly visually appealing.

(3) $\mathrm{PV} * \mathrm{SOL}-$ The main limitation of $\mathrm{PV} * \mathrm{SOL}$ is the high level of expertise required to perform a simulation, as exact system specifications need to be known. If not, it can be quite difficult to mix and match system sizing, inverters and cabling plans.

(4) Archelios - There is barely any documentation on Archelios, and there are no definitions for many of the parameters used in the software. Frequency of update of the software tool is unknown and leaves many questions unanswered. It is also poorly translated from French to English, and many of the variables are still in French.

(5) EnergyPlus - The main limitations with EnergyPlus is that it requires the usage of thirdparty software for 3D modeling and requires high level of expertise and is not userfriendly. 
(6) SAM - The 3D modeling environment is the main weakness in SAM. It is not capable of importing any CAD formats, and it can only draw four predefined simple shapes. There is no way to accurately model the exact geometry of any building in SAM's 3D interface at the current moment.

\subsubsection{Limitations and Errors}

This will be addressing the limitations and sources of errors to attributed to the benchmarking process of the solar simulation software tools. The case study area was modeled in HelioScope, PVsyst, PVSOL, Archelios, EnergyPlus, and SAM. Due to the inherent differences in the software features and capabilities, certain assumptions had to be made in the modeling approach. Determining the validity of a model is critical to providing confidence in the results. There is no standardized approach to validating PV models for comparing measured performance data with the performance predicted by a model (Stein et al., 2010). A standardized validation approach would allow for a process that compares measured actual PV performance data to estimated predictions that would help better understand and learn from the results (Klise \& Stein, 2009). There will always be uncertainty when it comes to modeling solar PV, since PV models rely on a given set of parameters to recreate the ability of a PV array system to produce electricity under a given set of site-specific meteorological conditions. The following is a summary of the main causes of limitations and errors in this study:

\section{(i) Weather data}

Although a weather sensor was installed at the case study location that measured GHI, it was not enough irradiance data in order to perform consistent simulations in the software tools. Sensor data was also unavailable for January 2018, due to calibration issues, and the sensor was uninstalled by December 5, 2018. Due to these issues, it was decided that the London CWEC 2016 EPW weather file would be best suited for the analysis. However, as EPW weather files represent a typical meteorological year and are derived from decades of averaged weather data, there was no way to accurately simulate the exact behaviour of the system in 2018. Ideally for a verification study, it is best to have the exact weather year. An ideal set for model validation 
would include total horizontal, horizontal diffuse, direct beam, and POA irradiance (Stein et al., 2010). This is what led to the deviations in the predictions made by the software tools.

(ii) Wiring of the PV system

The wiring mechanism in each software tool was different, hence there was no way to keep it $100 \%$ uniform. The default wiring mechanisms were used for each software tool.

(iii) Underlying mathematical assumptions used in the modeling tools

There is no way to keep this factor consistent, as these tools all have different models and mathematical assumptions. For example, the calculations of radiation on the POA (from the sky models), self-shading and horizon calculations and low light levels.

\section{(iv) Modeling of system losses}

The modeling of system losses can cause a huge impact on the generation potential of the system. There was no way to keep this process uniform as not all software tools offered the same parameters or capabilities for the modeling of system losses. Thus, the default values were used in the simulations, however, this could easily shift production estimate by over $5 \%$. There is a lack of data to make informed PV modeling derate assumptions in the industry, improvement of these assumptions is important to reduce PV modeling error (Freeman et al., 2014). Future studies for the development of accurate site-specific derate factors would be recommended to improve accuracies in the simulations.

\section{(v) Inability to model exact case study parameters in Archelios}

It was not possible to model the exact specifications of the case study area in Archelios, due to limitations of the software tools inverter modeling and PV stringing capabilities.

\section{(vi) Data for façade analysis and surrounding buildings}

Due to the limitations of the case study area of being only a single house, the surrounding effects of neighbouring buildings were not able to be modeled. Also, because there were no installations on the vertical facades of the house, the accuracy analysis of the software tools 
could not be examined on the 90-degree angle. Future work would be recommended in this area to enhance this study.

(vii) Financial, economical and environmental modeling

Due to limitations in the scope of the study and the availability of financial data for the case study area, the financial and economical considerations were not modeled. However, cost estimates (such as payback period), life cycle assessments and environmental benefits of PV systems (such as carbon dioxide emissions) are all important factors and should be investigated in future research to enhance this study.

\subsection{Software Tool Recommendations}

Based upon the results presented in Section 5.1 and Section 5.2, it is clear that there is no single software tool that has all the desired capabilities for the 3D modeling of solar communities. There are inherent strengths and weaknesses to each of the software tools and modeling methods presented. Based upon the results from the GIS modeling and the benchmarking of the solar simulation software tools, recommendations for the development of an integrated solution is presented. An ideal software tool for the modeling and mapping of 3D solar energy community potential should have the capabilities of GIS coupled with the modeling capabilities of these solar simulation software tools. An ideal tool would have the capabilities to:

- Data storage- have the capabilities of GIS for storing data in different layers for information on building materials, PV system sizing, inverter sizing etc.

- Be able to perform high resolution solar energy GIS mapping

- Be able to analyze rooftops and facades of buildings

- Have the accuracy of EnergyPlus for the simulation of PV performance

- Be available across different operating systems and opensource availability

- Be well documented and maintained

- Have a detailed weather database and accept importation formats in all the commonly used weather data files (like in PVsyst)

- Have numerous solar radiation sky model options available like in $\mathrm{PV} * \mathrm{SOL}$ 
- Important to have 3D CAD modeling capabilities compatible with popular formats of data (as exemplified by PV*SOL)

- The ability to perform detailed shade analysis based upon exact geometries of the buildings

- No restriction on the 3D modeling size or PV system sizing

- Have a built in PV and inverter component database that is updated frequently

- Have multiple PV performance and inverter modeling options (like in SAM)

- Detailed modeling of system loss parameters (PVsyst)

- To have detailed reporting and analysis options available

- To be user-friendly and not require too high of user expertise

- To provide recommendations for solar analysis

- Modeling flexibility to create own custom functions if desired

- Potential enhancement for future work with financial and economical modeling functions 


\section{CONCLUSIONS}

Solar PV energy offers a sustainable way of providing society with a renewable source of energy and can help decrease the reliance on fossil fuel consumption. It has been attracting increased attention in recent years due to the potential benefits it can provide towards a growing urban population, such as efficiency, economics and environmental impact. The objective of this thesis was to enhance the assessment ability of solar energy utilization and planning support for different types of buildings in a mixed-use community. New systems and methodologies with appropriate level of detail at lower computational time are needed to accurately model, estimate and map solar energy potential at a high spatiotemporal resolution. In order to accomplish this goal and to develop an integrated solution, solar potential was analyzed using two different types of studies: (1) 3D GIS modeling of a solar energy community, and (2) benchmarking of solar PV radiation software tools.

A 3D GIS method was used in order to develop an efficient method for urban solar mapping applications on an hourly basis to determine locations with maximum solar radiation potential, which would be ideal for PV installations. A model was created in ESRI ArcGIS 10.2,

to efficiently compute the hourly solar modeling and mapping process over a simulated year. The research was performed on a case study area located in southern Ontario, Canada. This study area is to be the location for a future net-zero solar energy community as proposed by the developer. Two different 3D models were mapped for solar potential and analyzed: (i) original site plan from the developers, and (ii) alternative solar community design developed by the collaborative research team formed by our industry partner. The analysis was tested at different resolutions $(0.05 \mathrm{~m}, 0.1 \mathrm{~m}, 0.5 \mathrm{~m}$, and $1 \mathrm{~m})$. A final pixelated resolution of $0.5 \mathrm{~m}$ was decided upon for the analysis due to computational time requirements. The designs were mapped on an hourly basis for all daylight hours within a simulated year, and a roof extraction procedure was created and employed in order to isolate the pixelated areas on the rooftops that are of particular interest for solar PV installation. This process generated a solar irradiation map for each daylight hour of the year approximating to over 2000 maps of the case study area for one scenario. This was a very computational and time intensive process, and all the hourly maps generated were stored in a geodatabase of over $32 \mathrm{~GB}$ in size. 
It was found that on an annual basis, the original site design and alternative design would receive approximately $874.9 \mathrm{kWh} / \mathrm{m}^{2}$ and $918.1 \mathrm{kwh} / \mathrm{m}^{2}$, respectively of solar radiation. That is a $4.7 \%$ increase in the amount of annual average solar radiation received. The maps were compared to the hourly solar maps generated for the original site plan for the winter and summer solstices. The winter and summer solstices received up to $6 \%$ and $14 \%$ more average solar radiation $\left(\mathrm{Wh} / \mathrm{m}^{2}\right)$, respectively. The alternative solar community design also provided less shading and average higher insolation values as compared to the original site plan. The accuracy of the work highly depended on the resolution and sky size of the input model. Future work is recommended in this area in order to create an efficient function for the automated extraction process of the pixelated solar radiation data.

Although GIS has very powerful capabilities for data storage and the mapping and modeling of solar potentials at a high spatiotemporal resolution, it is lacking in many functions to be considered as a sole tool for community energy PV analysis. It is a suitable tool for preliminary analysis to locate the hot spots for where you would receive the most irradiation, but with its limited capabilities for true 3D modeling (lack of vertical façade analysis), lack of an automated export function to generate the hourly irradiation results and functions for calculating PV potential, GIS on its own is not the best option. Hence, a more optimal solution for 3D solar energy community energy modeling is needed.

There are numerous solar simulation software tools that are widely available in the market. An assessment of these software tools is important to evaluate their strengths and weaknesses for performing analysis in different stages of the PV modeling process. The evaluation of the software tools was based upon their (i) accuracy in simulation performance analysis and (ii) comparison of their physical functions and capabilities. The software tools that were selected for evaluation were: HelioScope, PVsyst, PV*SOL, Archelios, EnergyPlus and SAM.

The six software tools were used to model a case study area located near London, Ontario for a net-zero energy residential home with an $8.745 \mathrm{~kW}$ PV system installation. Hourly PV generation data was collected for the case study area for the year of 2018, and the house was simulated in each of the software tools in 3D. The CWEC London EPW 2016 weather file was used for simulation. Multiple simulations were performed in each software tool under different transposition sky model options. For EnergyPlus, there was no option to change the 
transposition model, hence two different PV modeling options were evaluated instead. The hourly PV electricity results generated by Archelios were omitted from the quantitative performance analysis =because it was not able to replicate the exact system configuration of the case study area.

The measured annual PV generation for the case study area was found to be $9677.8 \mathrm{kWh}$. The software tool that was found to produce the most accurate estimates was EnergyPlus which modeled an annual percentage difference from $-0.6 \%-3.6 \%$. All the simulation software tools overpredicted the annual system yield, and PV*SOL was found to be the most aggressive with overestimates of up to $23.7 \%$. This is attributable to the fact that PV*SOL's default loss parameter modeling settings are lower than most of the software tools. Based upon the simulation results, the Perez model consistently produced the highest estimates in each of the software tools. The isotropic model in SAM and Liu \& Jordan model in PV*SOL produced the most conservative estimates within the software tools. The reasoning behind this is attributable to the fact that the Perez model is based upon anisotropic sky conditions, and this takes into consideration the realistic diffuse scattering effect of solar radiation for party cloudy skies. Whereas, for the isotropic models and the Liu \& Jordan isotropic model, they typically underrepresent the amount of solar radiation received on a surface from the diffuse component of solar radiation.

Another pattern that was evident was that all the software tools consistently overestimated generation in the winter months as compared to actual measured generation. This overestimation in generation is most likely due to snow cover in the winter months. Hence, improved snow models are recommended for the winter months in these software tools. Limitations to this study are due to the weather data and modeling assumptions (such as derate factors and PV wiring) that had to be made due to the inherent differences in software features and capabilities. Due to the lack of solar irradiance data for the case study area, the CWEC London 2016 weather file was used, which is representative of a typical meteorological year. Ideally, for a verification study, it is best to use data that is measured for the particular year. Future studies are recommended for the monitoring of weather data with multiple weather sensors (such as pyranometers and pyrheliometers) to produce high quality measurements for solar radiation data to try to replicate the conditions at the site as closely as possible. 
The second part of the benchmarking process was the determination and comparison of the physical functions and capabilities of the software tools. A list of fifteen functions and capabilities were presented for assessment in the six software tools: (1) system requirements and availability, (2) documentation and maintenance, (3) weather data, (4) solar radiation models, (5) 3D modeling capabilities, (6) shade modeling, (7) maximum modeling capabilities, (8) PV and inverter component databases, (9) PV performance model, (10) inverter model, (11) modeling of system losses, (12) cost of software tool, (13) report and analysis, (14) user-friendliness and level of expertise, and (15) modeling flexibility.

Based upon the comparison and evaluation of the software tools in each of the fifteen categories, the software tools that performed best in each category was determined. The software tool that performed the best in seven out of the fifteen criteria was found to be SAM, and the software tool that had the least functionality was Archelios, with a total score of two. However, each software tool had its strengths and weaknesses, and there was found to be no overall tool that had all the ideal functions and capabilities. The six software tools strengths and weaknesses are highlighted as follows:

- HelioScope was found to be a strong tool for its documentation and maintenance, shade modeling, report and analysis options, and user-friendliness and level of expertise. However, its main deficiency was in its 3D modeling capabilities.

- PVsyst was found to strong for its documentation and maintenance, weather data, shade modeling, system losses and report and analysis options, however, its main deficiency was its high level of expertise due to the complexity of its modeling parameters.

- $\quad$ PV*SOL has the strongest capabilities for 3D modeling, solar radiation modeling, and PV and inverter component databases. It also performs well for documentation and maintenance, shade modeling and report and analysis options. However, PV*SOL's main limitation is the level of expertise required in order to perform a simulation, as it requires detailed knowledge of exact system configurations and wiring.

- Archelios performed well in shade modeling and maximum modeling capabilities. However, it was found to be the least appealing software tool as many of its modeling parameters were unknown due to lack of documentation and transparency.

- EnergyPlus had its strengths in system requirements and availability, documentation and maintenance, shade modeling, maximum modeling capabilities, cost of software tool and 
modeling flexibility. However, its main deficiency is that it requires third-party software to be run for modeling in 3D and it is not user-friendly.

- SAM performed the best with strengths in system requirements and availability, documentation and maintenance, PV modeling, inverter modeling, cost of software tool, report and analysis, and modeling flexibility. However, its main limitation is its weakness in 3D modeling capabilities.

The main research contributions of this dissertation can be summarized as follows: the development of a 3D GIS modeling approach within ArcGIS to evaluate solar energy potential in a mixed-use community and the application of six solar PV radiation software tools to a residential home case study area to evaluate their strengths and weaknesses. This research also provided significant insights into how modeling and the application of software tools can be used to help plan and support the development of solar energy communities.

Based upon the work that has been presented in this dissertation, future research is recommended in the following areas:

- To further refine and enhance the benchmarking study by producing a weighted ranking scheme of the fifteen software tool physical functions and capabilities criteria and ranking of the importance in the benchmarking study

- An in-depth assessment of the performance and evaluation of the environmental, financial, and economical assessment of the solar PV software tools. For example, to perform a life cycle analysis and carbon emissions assessment comparative analysis.

- To investigate the effects of computational cost, resolution and simulation time

- Investigation of software tool accuracy with different geographic locations, weather files and climate types. For example, the development of a Canadian model for northern climates to provide a more robust model.

- Investigation of the accuracy of the software tools and shading capabilities using a case study area with measured PV generation data for multiple residential buildings as well as for façade analysis. Also, to potentially incorporate more solar PV software tools into the accuracy analysis.

- The program and development of a hybrid GIS simulation software tool coupled with the internal submodels from the software tools that were identified in this research to 
produce an all in one comprehensive simulation package for the mapping, modeling and simulation of solar PV electricity.

Despite great progress in the field, and increased computational power, this research has demonstrated that there are still many limitations to 3D solar community energy modeling and that there is the need for further enhancement and development of technologies and applications. Based upon the analyses performed using GIS for solar energy community modeling and mapping and the benchmarking of the six software tools HelioScope, PVsyst, PV*SOL, Archelios, EnergyPlus, and SAM, it is evident that there is not a single comprehensive software tool that has all the desired capabilities for the 3D modeling of solar PV communities. Since no one software tool clearly outperformed the others, the development of a future tool is recommended incorporating GIS with the internal radiation, PV module, inverter and shading submodels and functions that were identified from the various packages. Such a tool could pave the way for future developments as a key step to promoting the benefits of future PV and BIPV installations, net-zero solar energy communities and to help spur policy developments for a low carbon future. 


\section{APPENDICES}

Appendix A. Example of GIS input parameters for Area Solar Radiation Model used for the month of January.

\begin{tabular}{|c|c|c|c|c|c|c|c|c|c|c|c|c|c|c|c|}
\hline 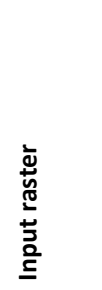 & 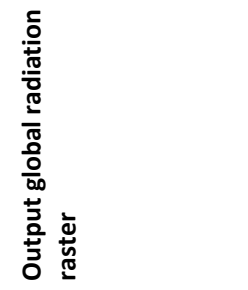 & 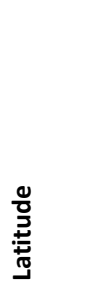 & 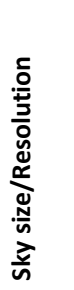 & 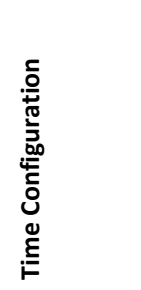 & 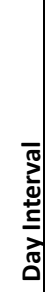 & 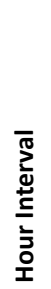 & 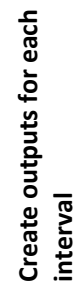 & 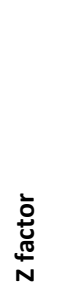 & 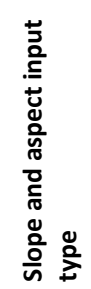 & 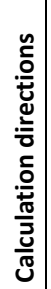 & 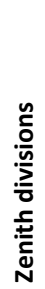 & 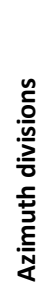 & 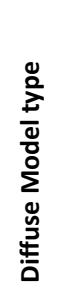 & 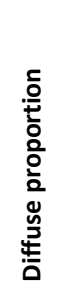 & 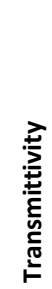 \\
\hline $\begin{array}{l}\text { Combi } \\
\text { ned_0. } \\
5 \mathrm{~m} . \text { tif }\end{array}$ & 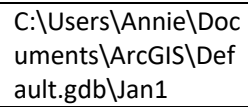 & $\begin{array}{r}42.983 \\
7\end{array}$ & 200 & $\begin{array}{l}\text { WithinDay } \\
1024\end{array}$ & 1 & 1 & TRUE & 1 & $\begin{array}{l}\text { FROM } \\
\text { _DEM }\end{array}$ & $\begin{array}{l}3 \\
2\end{array}$ & 8 & 8 & $\begin{array}{r}\text { UNIF } \\
\text { ORM } \\
\text { SKY }\end{array}$ & 0.3 & 0.5 \\
\hline $\begin{array}{l}\text { Combi } \\
\text { ned_0. } \\
5 m . t i f\end{array}$ & 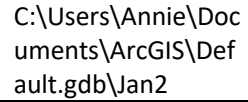 & $\begin{array}{r}42.983 \\
7\end{array}$ & 200 & $\begin{array}{l}\text { WithinDay } \\
2024\end{array}$ & 1 & 1 & TRUE & 1 & $\begin{array}{l}\text { FROM } \\
\text { _DEM }\end{array}$ & $\begin{array}{l}3 \\
2\end{array}$ & 8 & 8 & $\begin{array}{l}\text { UNIF } \\
\text { ORM } \\
\text { SKY }\end{array}$ & 0.3 & 0.5 \\
\hline $\begin{array}{l}\text { Combi } \\
\text { ned_0. } \\
5 m . \text { tif }\end{array}$ & 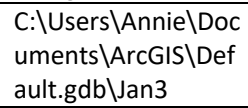 & $\begin{array}{r}42.983 \\
7\end{array}$ & 200 & $\begin{array}{l}\text { WithinDay } \\
3024\end{array}$ & 1 & 1 & TRUE & 1 & $\begin{array}{l}\text { FROM } \\
\text { _DEM }\end{array}$ & $\begin{array}{l}3 \\
2\end{array}$ & 8 & 8 & $\begin{array}{l}\text { UNIF } \\
\text { ORM } \\
\text { SKY }\end{array}$ & 0.3 & 0.5 \\
\hline $\begin{array}{l}\text { Combi } \\
\text { ned_0. } \\
5 \mathrm{~m} . \text { tif } \\
\end{array}$ & 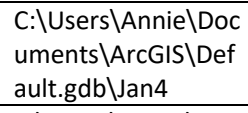 & $\begin{array}{r}42.983 \\
7\end{array}$ & 200 & $\begin{array}{l}\text { WithinDay } \\
4024\end{array}$ & 1 & 1 & TRUE & 1 & $\begin{array}{l}\text { FROM } \\
\text { _DEM }\end{array}$ & $\begin{array}{l}3 \\
2\end{array}$ & 8 & 8 & $\begin{array}{r}\text { UNIF } \\
\text { ORM } \\
\text { SKY } \\
\end{array}$ & 0.3 & 0.5 \\
\hline $\begin{array}{l}\text { Combi } \\
\text { ned_0. } \\
5 \mathrm{~m} . \text { tif }\end{array}$ & 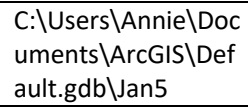 & $\begin{array}{r}42.983 \\
7\end{array}$ & 200 & $\begin{array}{l}\text { WithinDay } \\
5024\end{array}$ & 1 & 1 & TRUE & 1 & $\begin{array}{l}\text { FROM } \\
\text { _DEM }\end{array}$ & $\begin{array}{l}3 \\
2\end{array}$ & 8 & 8 & $\begin{array}{l}\text { UNIF } \\
\text { ORM } \\
\text { SKY }\end{array}$ & 0.3 & 0.5 \\
\hline $\begin{array}{l}\text { Combi } \\
\text { ned_0. } \\
5 \mathrm{~m} . \text { tif }\end{array}$ & 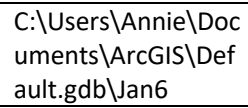 & $\begin{array}{r}42.983 \\
7\end{array}$ & 200 & $\begin{array}{l}\text { WithinDay } \\
6024\end{array}$ & 1 & 1 & TRUE & 1 & $\begin{array}{l}\text { FROM } \\
\text { _DEM }\end{array}$ & $\begin{array}{l}3 \\
2\end{array}$ & 8 & 8 & $\begin{array}{l}\text { UNIF } \\
\text { ORM } \\
\text { SKY }\end{array}$ & 0.3 & 0.5 \\
\hline $\begin{array}{l}\text { Combi } \\
\text { ned_0. } \\
5 m . t i f\end{array}$ & 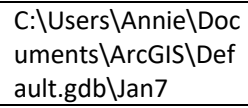 & $\begin{array}{r}42.983 \\
7\end{array}$ & 200 & $\begin{array}{l}\text { WithinDay } \\
7024\end{array}$ & 1 & 1 & TRUE & 1 & $\begin{array}{l}\text { FROM } \\
\text { _DEM }\end{array}$ & $\begin{array}{l}3 \\
2\end{array}$ & 8 & 8 & $\begin{array}{l}\text { UNIF } \\
\text { ORM } \\
\text { SKY }\end{array}$ & 0.3 & 0.5 \\
\hline $\begin{array}{l}\text { Combi } \\
\text { ned_0. } \\
5 \mathrm{~m} . \mathrm{tif}\end{array}$ & $\begin{array}{l}\text { C: \Users \Annie\Doc } \\
\text { uments } \backslash \text { ArcGIS\Def } \\
\text { ault.gdb } \backslash \text { Jan8 }\end{array}$ & $\begin{array}{r}42.983 \\
7\end{array}$ & 200 & $\begin{array}{l}\text { WithinDay } \\
8024\end{array}$ & 1 & 1 & TRUE & 1 & $\begin{array}{r}\text { FROM } \\
\text { _DEM }\end{array}$ & $\begin{array}{l}3 \\
2\end{array}$ & 8 & 8 & $\begin{array}{r}\text { UNIF } \\
\text { ORM } \\
\text { SKY }\end{array}$ & 0.3 & 0.5 \\
\hline $\begin{array}{l}\text { Combi } \\
\text { ned_0. } \\
5 \mathrm{~m} . \text { tif }\end{array}$ & 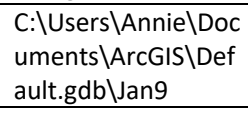 & $\begin{array}{r}42.983 \\
7\end{array}$ & 200 & $\begin{array}{l}\text { WithinDay } \\
9024\end{array}$ & 1 & 1 & TRUE & 1 & $\begin{array}{l}\text { FROM } \\
\text { _DEM }\end{array}$ & $\begin{array}{l}3 \\
2\end{array}$ & 8 & 8 & $\begin{array}{r}\text { UNIF } \\
\text { ORM } \\
\text { SKY }\end{array}$ & 0.3 & 0.5 \\
\hline $\begin{array}{l}\text { Combi } \\
\text { ned_0. } \\
5 \mathrm{~m} . \text { tif }\end{array}$ & $\begin{array}{l}\text { C: \Users } \backslash A n n i e \backslash \text { Doc } \\
\text { uments } \backslash \text { ArcGIS } \backslash \text { Def } \\
\text { ault.gdb } \backslash \operatorname{Jan} 10\end{array}$ & $\begin{array}{r}42.983 \\
7\end{array}$ & 200 & $\begin{array}{l}\text { WithinDay } \\
10024\end{array}$ & 1 & 1 & TRUE & 1 & $\begin{array}{l}\text { FROM } \\
\text { _DEM }\end{array}$ & $\begin{array}{l}3 \\
2\end{array}$ & 8 & 8 & $\begin{array}{r}\text { UNIF } \\
\text { ORM } \\
\text { SKY }\end{array}$ & 0.3 & 0.5 \\
\hline $\begin{array}{l}\text { Combi } \\
\text { ned_0. } \\
5 \mathrm{~m} . \text { tif } \\
\end{array}$ & $\begin{array}{l}\text { C: \Users\Annie\Doc } \\
\text { uments } \backslash A r c G I S \backslash \text { Def } \\
\text { ault.gdb } \backslash \operatorname{Jan} 11\end{array}$ & $\begin{array}{r}42.983 \\
7\end{array}$ & 200 & $\begin{array}{l}\text { WithinDay } \\
11024\end{array}$ & 1 & 1 & TRUE & 1 & $\begin{array}{l}\text { FROM } \\
\text { _DEM }\end{array}$ & $\begin{array}{l}3 \\
2\end{array}$ & 8 & 8 & $\begin{array}{r}\text { UNIF } \\
\text { ORM } \\
\text { SKY } \\
\end{array}$ & 0.3 & 0.5 \\
\hline $\begin{array}{l}\text { Combi } \\
\text { ned_0. } \\
5 \mathrm{~m} . \text { tif }\end{array}$ & $\begin{array}{l}\text { C: \Users \Annie\Doc } \\
\text { uments } \backslash \text { ArcGIS\Def } \\
\text { ault.gdb } \backslash \operatorname{Jan} 12\end{array}$ & $\begin{array}{r}42.983 \\
7\end{array}$ & 200 & $\begin{array}{l}\text { WithinDay } \\
12024\end{array}$ & 1 & 1 & TRUE & 1 & $\begin{array}{l}\text { FROM } \\
\text { _DEM }\end{array}$ & $\begin{array}{l}3 \\
2\end{array}$ & 8 & 8 & $\begin{array}{r}\text { UNIF } \\
\text { ORM } \\
\text { SKY }\end{array}$ & 0.3 & 0.5 \\
\hline $\begin{array}{l}\text { Combi } \\
\text { ned_0. } \\
5 \mathrm{~m} . \text { tif } \\
\end{array}$ & 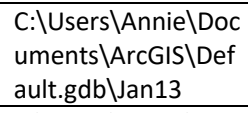 & $\begin{array}{r}42.983 \\
7\end{array}$ & 200 & $\begin{array}{l}\text { WithinDay } \\
13024\end{array}$ & 1 & 1 & TRUE & 1 & $\begin{array}{l}\text { FROM } \\
\text { _DEM }\end{array}$ & $\begin{array}{l}3 \\
2\end{array}$ & 8 & 8 & $\begin{array}{r}\text { UNIF } \\
\text { ORM } \\
\text { SKY }\end{array}$ & 0.3 & 0.5 \\
\hline $\begin{array}{l}\text { Combi } \\
\text { ned_0. } \\
5 \mathrm{~m} . \text { tif } \\
\end{array}$ & $\begin{array}{l}\text { C: \Users \Annie\Doc } \\
\text { uments } \backslash A r c G I S \backslash \text { Def } \\
\text { ault.gdb } \backslash \operatorname{Jan} 14\end{array}$ & $\begin{array}{r}42.983 \\
7\end{array}$ & 200 & $\begin{array}{l}\text { WithinDay } \\
14024\end{array}$ & 1 & 1 & TRUE & 1 & $\begin{array}{l}\text { FROM } \\
\text { _DEM }\end{array}$ & $\begin{array}{l}3 \\
2\end{array}$ & 8 & 8 & $\begin{array}{r}\text { UNIF } \\
\text { ORM } \\
\text { SKY }\end{array}$ & 0.3 & 0.5 \\
\hline $\begin{array}{l}\text { Combi } \\
\text { ned_0. } \\
5 m . t i f\end{array}$ & $\begin{array}{l}\text { C: \Users\Annie\Doc } \\
\text { uments\ArcGIS\Def } \\
\text { ault.gdb\Jan15 }\end{array}$ & $\begin{array}{r}42.983 \\
7\end{array}$ & 200 & $\begin{array}{l}\text { WithinDay } \\
15024\end{array}$ & 1 & 1 & TRUE & 1 & $\begin{array}{r}\text { FROM } \\
\text { _DEM }\end{array}$ & $\begin{array}{l}3 \\
2\end{array}$ & 8 & 8 & $\begin{array}{l}\text { UNIF } \\
\text { ORM } \\
\text { SKY }\end{array}$ & 0.3 & 0.5 \\
\hline $\begin{array}{l}\text { Combi } \\
\text { ned_0. } \\
5 \mathrm{~m} . \text { tif }\end{array}$ & 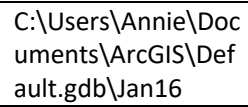 & $\begin{array}{r}42.983 \\
7\end{array}$ & 200 & $\begin{array}{l}\text { WithinDay } \\
16024\end{array}$ & 1 & 1 & TRUE & 1 & $\begin{array}{l}\text { FROM } \\
\text { _DEM }\end{array}$ & $\begin{array}{l}3 \\
2\end{array}$ & 8 & 8 & $\begin{array}{l}\text { UNIF } \\
\text { ORM } \\
\text { SKY }\end{array}$ & 0.3 & 0.5 \\
\hline
\end{tabular}




\begin{tabular}{|c|c|c|c|c|c|c|c|c|c|c|c|c|c|c|c|}
\hline $\begin{array}{l}\text { Combi } \\
\text { ned_0. } \\
5 \mathrm{~m} . \text { tif }\end{array}$ & 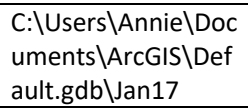 & $\begin{array}{r}42.983 \\
7\end{array}$ & 200 & $\begin{array}{l}\text { WithinDay } \\
17024\end{array}$ & 1 & 1 & TRUE & 1 & $\begin{array}{l}\text { FROM } \\
\text { _DEM }\end{array}$ & $\begin{array}{l}3 \\
2\end{array}$ & 8 & 8 & $\begin{array}{l}\text { UNIF } \\
\text { ORM } \\
\text { SKY }\end{array}$ & 0.3 & 0.5 \\
\hline $\begin{array}{l}\text { Combi } \\
\text { ned_0. } \\
5 \mathrm{~m} . \mathrm{tif}\end{array}$ & 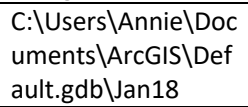 & $\begin{array}{r}42.983 \\
7\end{array}$ & 200 & $\begin{array}{l}\text { WithinDay } \\
18024\end{array}$ & 1 & 1 & TRUE & 1 & $\begin{array}{l}\text { FROM } \\
\text { _DEM }\end{array}$ & $\begin{array}{l}3 \\
2\end{array}$ & 8 & 8 & $\begin{array}{l}\text { UNIF } \\
\text { ORM } \\
\text { SKY }\end{array}$ & 0.3 & 0.5 \\
\hline $\begin{array}{l}\text { Combi } \\
\text { ned_0. } \\
5 \mathrm{~m} . \text { tif }\end{array}$ & $\begin{array}{l}\text { C: } \backslash \text { Users } \backslash A n n i e \backslash D o c \\
\text { uments\ArcGIS\Def } \\
\text { ault.gdb\Jan19 }\end{array}$ & $\begin{array}{r}42.983 \\
7\end{array}$ & 200 & $\begin{array}{l}\text { WithinDay } \\
19024\end{array}$ & 1 & 1 & TRUE & 1 & $\begin{array}{l}\text { FROM } \\
\text { _DEM }\end{array}$ & $\begin{array}{l}3 \\
2\end{array}$ & 8 & 8 & $\begin{array}{l}\text { UNIF } \\
\text { ORM } \\
\text { SKY }\end{array}$ & 0.3 & 0.5 \\
\hline $\begin{array}{l}\text { Combi } \\
\text { ned_0. } \\
5 \mathrm{~m} . \text { tif }\end{array}$ & $\begin{array}{l}\text { C: } \backslash \text { Users } \backslash A n n i e \backslash \text { Doc } \\
\text { uments\ArcGIS\Def } \\
\text { ault.gdb\Jan20 }\end{array}$ & $\begin{array}{r}42.983 \\
7\end{array}$ & 200 & $\begin{array}{l}\text { WithinDay } \\
20024\end{array}$ & 1 & 1 & TRUE & 1 & $\begin{array}{l}\text { FROM } \\
\text { _DEM }\end{array}$ & $\begin{array}{l}3 \\
2\end{array}$ & 8 & 8 & $\begin{array}{l}\text { UNIF } \\
\text { ORM } \\
\text { SKYY }\end{array}$ & 0.3 & 0.5 \\
\hline $\begin{array}{l}\text { Combi } \\
\text { ned_0. } \\
5 m . t i f\end{array}$ & $\begin{array}{l}\text { C: } \backslash \text { Users } \backslash A n n i e \backslash \text { Doc } \\
\text { uments\ArcGIS\Def } \\
\text { ault.gdb\Jan21 }\end{array}$ & $\begin{array}{r}42.983 \\
7\end{array}$ & 200 & $\begin{array}{l}\text { WithinDay } \\
21024\end{array}$ & 1 & 1 & TRUE & 1 & $\begin{array}{l}\text { FROM } \\
\text { DEEM }\end{array}$ & $\begin{array}{l}3 \\
2\end{array}$ & 8 & 8 & $\begin{array}{l}\text { UNIF } \\
\text { ORM } \\
\text { SKYY }\end{array}$ & 0.3 & 0.5 \\
\hline $\begin{array}{l}\text { Combi } \\
\text { ned_0. } \\
5 \mathrm{~m} . \text { tif }\end{array}$ & $\begin{array}{l}\text { C: } \backslash \text { Users } \backslash A n n i e \backslash D o c \\
\text { uments\ArcGIS\Def } \\
\text { ault.gdb\Jan22 }\end{array}$ & $\begin{array}{r}42.983 \\
7\end{array}$ & 200 & $\begin{array}{l}\text { WithinDay } \\
22024\end{array}$ & 1 & 1 & TRUE & 1 & $\begin{array}{l}\text { FROM } \\
\text { DEEM }\end{array}$ & $\begin{array}{l}3 \\
2\end{array}$ & 8 & 8 & $\begin{array}{l}\text { UNIF } \\
\text { ORM } \\
\text { SKYY }\end{array}$ & 0.3 & 0.5 \\
\hline $\begin{array}{l}\text { Combi } \\
\text { ned_0. } \\
5 \mathrm{~m} . \text { tif }\end{array}$ & 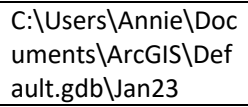 & $\begin{array}{r}42.983 \\
7\end{array}$ & 200 & $\begin{array}{l}\text { WithinDay } \\
23024\end{array}$ & 1 & 1 & TRUE & 1 & $\begin{array}{l}\text { FROM } \\
\text { _DEM }\end{array}$ & $\begin{array}{l}3 \\
2\end{array}$ & 8 & 8 & $\begin{array}{l}\text { UNIF } \\
\text { ORM } \\
\text { SKYY }\end{array}$ & 0.3 & 0.5 \\
\hline $\begin{array}{l}\text { Combi } \\
\text { ned_0. } \\
5 m . t i f\end{array}$ & $\begin{array}{l}\text { C: } \backslash \text { Users } \backslash A n n i e \backslash \text { Doc } \\
\text { uments\ArcGIS\Def } \\
\text { ault.gdb\Jan24 }\end{array}$ & $\begin{array}{r}42.983 \\
7\end{array}$ & 200 & $\begin{array}{l}\text { WithinDay } \\
24024\end{array}$ & 1 & 1 & TRUE & 1 & $\begin{array}{l}\text { FROM } \\
\text { _DEM }\end{array}$ & $\begin{array}{l}3 \\
2\end{array}$ & 8 & 8 & $\begin{array}{l}\text { UNIF } \\
\text { ORM } \\
\text { SKY }\end{array}$ & 0.3 & 0.5 \\
\hline $\begin{array}{l}\text { Combi } \\
\text { ned_0. } \\
5 \mathrm{~m} . \text { tif }\end{array}$ & $\begin{array}{l}\text { C: } \backslash \text { Users } \backslash A n n i e \backslash \text { Doc } \\
\text { uments\ArcGIS\Def } \\
\text { ault.gdb\Jan25 }\end{array}$ & $\begin{array}{r}42.983 \\
7\end{array}$ & 200 & $\begin{array}{l}\text { WithinDay } \\
25024\end{array}$ & 1 & 1 & TRUE & 1 & $\begin{array}{l}\text { FROM } \\
\text { _DEM }\end{array}$ & $\begin{array}{l}3 \\
2\end{array}$ & 8 & 8 & $\begin{array}{l}\text { UNIF } \\
\text { ORM } \\
\text { SKYY }\end{array}$ & 0.3 & 0.5 \\
\hline $\begin{array}{l}\text { Combi } \\
\text { ned_0. } \\
5 \mathrm{~m} . \text { tif }\end{array}$ & 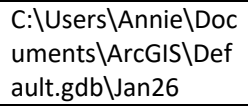 & $\begin{array}{r}42.983 \\
7\end{array}$ & 200 & $\begin{array}{l}\text { WithinDay } \\
26024\end{array}$ & 1 & 1 & TRUE & 1 & $\begin{array}{l}\text { FROM } \\
\text { _DEM }\end{array}$ & $\begin{array}{l}3 \\
2\end{array}$ & 8 & 8 & $\begin{array}{l}\text { UNIF } \\
\text { ORM } \\
\text { SKYY }\end{array}$ & 0.3 & 0.5 \\
\hline $\begin{array}{l}\text { Combi } \\
\text { ned_0. } \\
5 \mathrm{~m} . \text { tif }\end{array}$ & 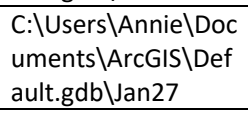 & $\begin{array}{r}42.983 \\
7\end{array}$ & 200 & $\begin{array}{l}\text { WithinDay } \\
27024\end{array}$ & 1 & 1 & TRUE & 1 & $\begin{array}{l}\text { FROM } \\
\text { _DEM }\end{array}$ & $\begin{array}{l}3 \\
2\end{array}$ & 8 & 8 & $\begin{array}{l}\text { UNIF } \\
\text { ORM } \\
\text { SKY }\end{array}$ & 0.3 & 0.5 \\
\hline $\begin{array}{l}\text { Combi } \\
\text { ned_0. } \\
5 \mathrm{~m} . \text { tif }\end{array}$ & $\begin{array}{l}\text { C:\Users \Annie\Doc } \\
\text { uments } \backslash \text { ArcGIS\Def } \\
\text { ault.gdb\Jan28 }\end{array}$ & $\begin{array}{r}42.983 \\
7\end{array}$ & 200 & $\begin{array}{l}\text { WithinDay } \\
28024\end{array}$ & 1 & 1 & TRUE & 1 & $\begin{array}{l}\text { FROM } \\
\text { _DEM }\end{array}$ & $\begin{array}{l}3 \\
2\end{array}$ & 8 & 8 & $\begin{array}{l}\text { UNIF } \\
\text { ORM } \\
\text { SKY } \\
\end{array}$ & 0.3 & 0.5 \\
\hline $\begin{array}{l}\text { Combi } \\
\text { ned_0. } \\
5 \mathrm{~m} . \text { tif }\end{array}$ & $\begin{array}{l}\text { C:\Users \Annie\Doc } \\
\text { uments\ArcGIS\Def } \\
\text { ault.gdb\Jan29 }\end{array}$ & $\begin{array}{r}42.983 \\
7\end{array}$ & 200 & $\begin{array}{l}\text { WithinDay } \\
29024\end{array}$ & 1 & 1 & TRUE & 1 & $\begin{array}{l}\text { FROM } \\
\text { _DEM }\end{array}$ & $\begin{array}{l}3 \\
2\end{array}$ & 8 & 8 & $\begin{array}{r}\text { UNIF } \\
\text { ORM } \\
\text { SKY }\end{array}$ & 0.3 & 0.5 \\
\hline $\begin{array}{l}\text { Combi } \\
\text { ned_0. } \\
5 \mathrm{~m} . \text { tif }\end{array}$ & $\begin{array}{l}\text { C: } \backslash \text { Users } \backslash A n n i e \backslash D o c \\
\text { uments\ArcGIS\Def } \\
\text { ault.gdb\Jan30 }\end{array}$ & $\begin{array}{r}42.983 \\
7\end{array}$ & 200 & $\begin{array}{l}\text { WithinDay } \\
30024\end{array}$ & 1 & 1 & TRUE & 1 & $\begin{array}{l}\text { FROM } \\
\text { DEEM }\end{array}$ & $\begin{array}{l}3 \\
2\end{array}$ & 8 & 8 & $\begin{array}{l}\text { UNIF } \\
\text { ORM } \\
\text { SKKY }\end{array}$ & 0.3 & 0.5 \\
\hline $\begin{array}{l}\text { Combi } \\
\text { ned_0. } \\
5 \mathrm{~m} . \text { tif }\end{array}$ & $\begin{array}{l}\text { C: } \backslash \text { Users } \backslash A n n i e \backslash D o c \\
\text { uments\ArcGIS\Def } \\
\text { ault.gdb\Jan31 }\end{array}$ & $\begin{array}{r}42.983 \\
7\end{array}$ & 200 & $\begin{array}{l}\text { WithinDay } \\
31024\end{array}$ & 1 & 1 & TRUE & 1 & $\begin{array}{l}\text { FROM } \\
\text { _DEM }\end{array}$ & $\begin{array}{l}3 \\
2\end{array}$ & 8 & 8 & $\begin{array}{l}\text { UNIF } \\
\text { ORM } \\
\text { SKY }\end{array}$ & 0.3 & 0.5 \\
\hline
\end{tabular}




\section{UHelioScope}

Case Study Area Single House (corrected) Case Study Area, 247 Rushby St, Strathroy,

Ontario, Canada

\begin{tabular}{|l|l|}
\hline \multicolumn{1}{|c|}{ Report } \\
\hline Project Name & Case Study Area \\
\hline Project Address & 247 Rushby St, Strathroy, Ontario, Canada \\
\hline Prepared By & $\begin{array}{l}\text { Annie Chow } \\
\text { annie.chow@ryerson.ca }\end{array}$ \\
\hline
\end{tabular}

Llll Monthly Production

$$
1500
$$

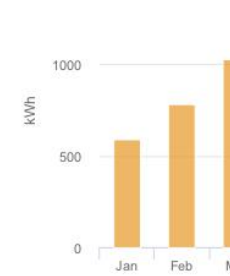

4 Annual Production

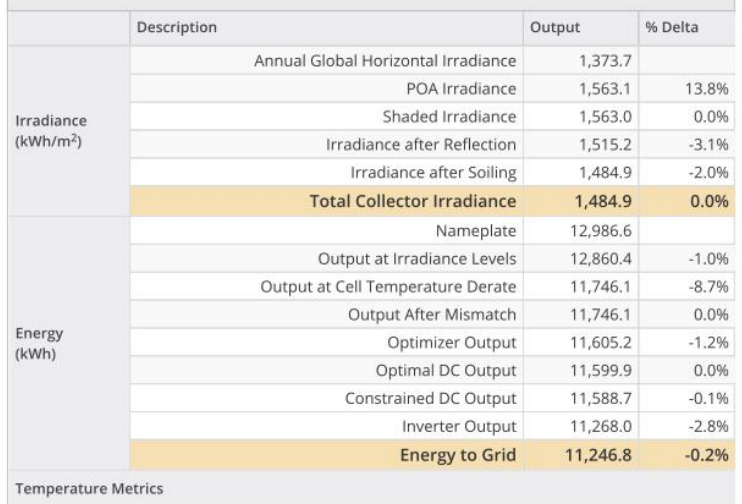

Temperature Metrics

Avg. Operating Ambient Temp Avg, Operating Cell Temp Simulation Metrics

\begin{tabular}{|l|l|}
\hline \multicolumn{2}{|l|}{ Llll System Metrics } \\
\hline Design & Case Study Area Single House (corrected) \\
\hline $\begin{array}{l}\text { Module DC } \\
\text { Nameplate }\end{array}$ & $8.75 \mathrm{~kW}$ \\
\hline $\begin{array}{l}\text { Inverter AC } \\
\text { Nameplate }\end{array}$ & $\begin{array}{l}7.63 \mathrm{~kW} \\
\text { Load Ratio: } 1.15\end{array}$ \\
\hline $\begin{array}{l}\text { Annual } \\
\text { Production }\end{array}$ & $11.25 \mathrm{MWh}$ \\
\hline $\begin{array}{l}\text { Performance } \\
\text { Ratio }\end{array}$ & $82.3 \%$ \\
\hline kWh/kWp & $1,286.1$ \\
\hline Weather Dataset & TMY, LONDON A, CWEC2016 (custom) \\
\hline Simulator Version & $\begin{array}{l}703 \mathrm{~b} 6 \mathrm{~d} 12 \mathrm{ad}-86 \mathrm{c} 29 \mathrm{~b} 30 \mathrm{bc}-5 \mathrm{~b} 2 \mathrm{a} 11 \mathrm{c} 77 \mathrm{a}- \\
\text { ae1226210e }\end{array}$ \\
\hline
\end{tabular}

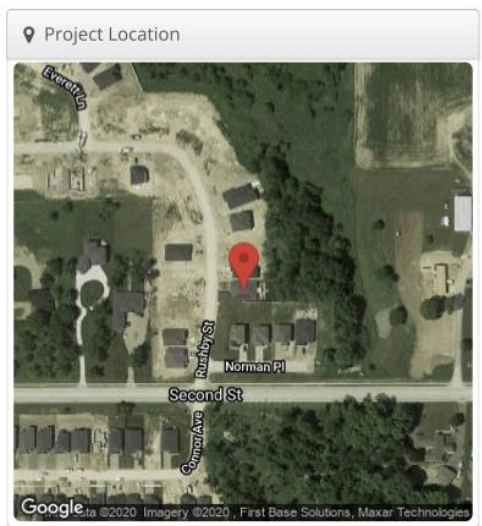

- Sources of System Loss

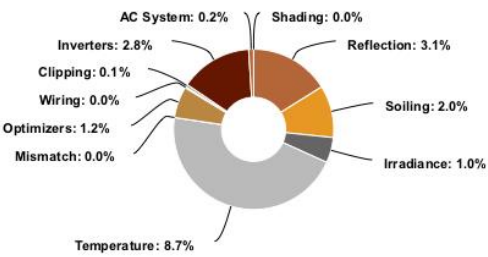




\section{HelioScope}

\begin{tabular}{|c|c|c|c|c|c|c|c|c|c|c|c|c|}
\hline Description & \multicolumn{12}{|c|}{ Condition Set 1} \\
\hline Weather Dataset & \multicolumn{12}{|c|}{ TMY, LONDON A, CWEC2016 (custom) } \\
\hline Solar Angle Location & \multicolumn{12}{|c|}{ Meteo Lat/Lng } \\
\hline Transposition Model & \multicolumn{12}{|c|}{ Perez Model } \\
\hline Temperature Model & \multicolumn{12}{|c|}{ Sandia Model } \\
\hline \multirow{3}{*}{$\begin{array}{l}\text { Temperature Model } \\
\text { Parameters }\end{array}$} & \multicolumn{3}{|c|}{ Rack Type } & \multicolumn{2}{|l|}{ a } & \multicolumn{2}{|l|}{ b } & \multicolumn{5}{|c|}{ Temperature Delta } \\
\hline & \multicolumn{3}{|c|}{ Fixed Tilt } & \multicolumn{2}{|c|}{-3.56} & \multicolumn{2}{|c|}{-0.075} & \multicolumn{5}{|c|}{$3^{\circ} \mathrm{C}$} \\
\hline & \multicolumn{3}{|c|}{ Flush Mount } & \multicolumn{2}{|c|}{-2.81} & \multicolumn{2}{|c|}{-0.0455} & \multicolumn{5}{|c|}{$0^{\circ} \mathrm{C}$} \\
\hline \multirow{2}{*}{ Soiling (\%) } & J & $\mathrm{F}$ & M & A & M & J & J & A & $\mathrm{s}$ & 0 & $\mathrm{~N}$ & D \\
\hline & 2 & 2 & 2 & 2 & 2 & 2 & 2 & 2 & 2 & 2 & 2 & 2 \\
\hline Irradiation Variance & \multicolumn{12}{|l|}{$5 \%$} \\
\hline $\begin{array}{l}\text { Cell Temperature } \\
\text { Spread }\end{array}$ & \multicolumn{12}{|c|}{$4^{\circ} \mathrm{C}$} \\
\hline Module Binning Range & \multicolumn{12}{|c|}{$-2.5 \%$ to $2.5 \%$} \\
\hline AC System Derate & \multicolumn{12}{|c|}{$0.50 \%$} \\
\hline \multirow{2}{*}{$\begin{array}{l}\text { Module } \\
\text { Characterizations }\end{array}$} & \multicolumn{3}{|c|}{ Module } & \multicolumn{2}{|c|}{$\begin{array}{l}\text { Uploaded } \\
\text { By }\end{array}$} & \multicolumn{7}{|c|}{ Characterization } \\
\hline & \multicolumn{3}{|c|}{$\begin{array}{l}\text { CS6P - 265P } \\
\text { (Canadian Solar } \\
\text { Inc.) }\end{array}$} & \multicolumn{2}{|c|}{$\begin{array}{l}\text { Folsom } \\
\text { Labs }\end{array}$} & \multicolumn{7}{|c|}{$\begin{array}{l}\text { CS6P- } \\
\text { 265P_MIX_CSI_EXT_V6.44_2016Q2.PAN, } \\
\text { PAN }\end{array}$} \\
\hline & \multicolumn{5}{|c|}{ Device } & \multicolumn{3}{|c|}{ Uploaded By } & Chara & teriz. & & \\
\hline $\begin{array}{l}\text { Component } \\
\text { Characterizations }\end{array}$ & $\begin{array}{l}\text { SE76 } \\
\text { (Sola }\end{array}$ & Edg & $(240 \mathrm{~V}$ & & & & & & $\begin{array}{l}\text { Defat } \\
\text { Char: }\end{array}$ & teriz & & \\
\hline & P30C & (Sol & Edge) & & & & som & & Mfg S & ec $s$ & & \\
\hline
\end{tabular}

\begin{tabular}{|c|c|c|}
\hline Component & Name & Count \\
\hline Inverters & SE7600A-US (240V) (SolarEdge) & $\begin{array}{l}1(7.63 \\
k W)\end{array}$ \\
\hline $\begin{array}{l}\text { AC Home } \\
\text { Runs }\end{array}$ & 8 AWG (Copper) & $1(18.5 \mathrm{~m})$ \\
\hline Strings & 10 AWG (Copper) & $2(7.5 \mathrm{~m})$ \\
\hline Optimizers & P300 (SolarEdge) & $\begin{array}{l}33(9.90 \\
k W)\end{array}$ \\
\hline Module & $\begin{array}{l}\text { Canadian Solar Inc., CS6P - 265P } \\
(265 \mathrm{~W})\end{array}$ & $\begin{array}{l}33(8.75 \\
\mathrm{kW})\end{array}$ \\
\hline
\end{tabular}

\begin{tabular}{|c|c|c|c|c|c|c|c|c|c|}
\hline \multicolumn{10}{|c|}{ 호 Wiring Zones } \\
\hline Description & \multicolumn{3}{|c|}{ Combiner Poles } & \multicolumn{2}{|c|}{ String Size } & \multicolumn{4}{|c|}{ Stringing Strategy } \\
\hline Wiring Zone & \multicolumn{2}{|l|}{12} & \multicolumn{3}{|c|}{ 8-19 } & \multicolumn{4}{|c|}{ Along Racking } \\
\hline \multicolumn{10}{|c|}{ III Field Segments } \\
\hline Description & Racking & Orientation & Tilt & Azimuth & Intrarow Spacing & Frame Size & Frames & Modules & Power \\
\hline Field Segment 1 & Flush Mount & Portrait (Vertical) & $33.69^{\circ}$ & $180^{\circ}$ & $0.0 \mathrm{~m}$ & $1 \times 1$ & 77 & 33 & $8.75 \mathrm{~kW}$ \\
\hline
\end{tabular}




\section{HelioScope}

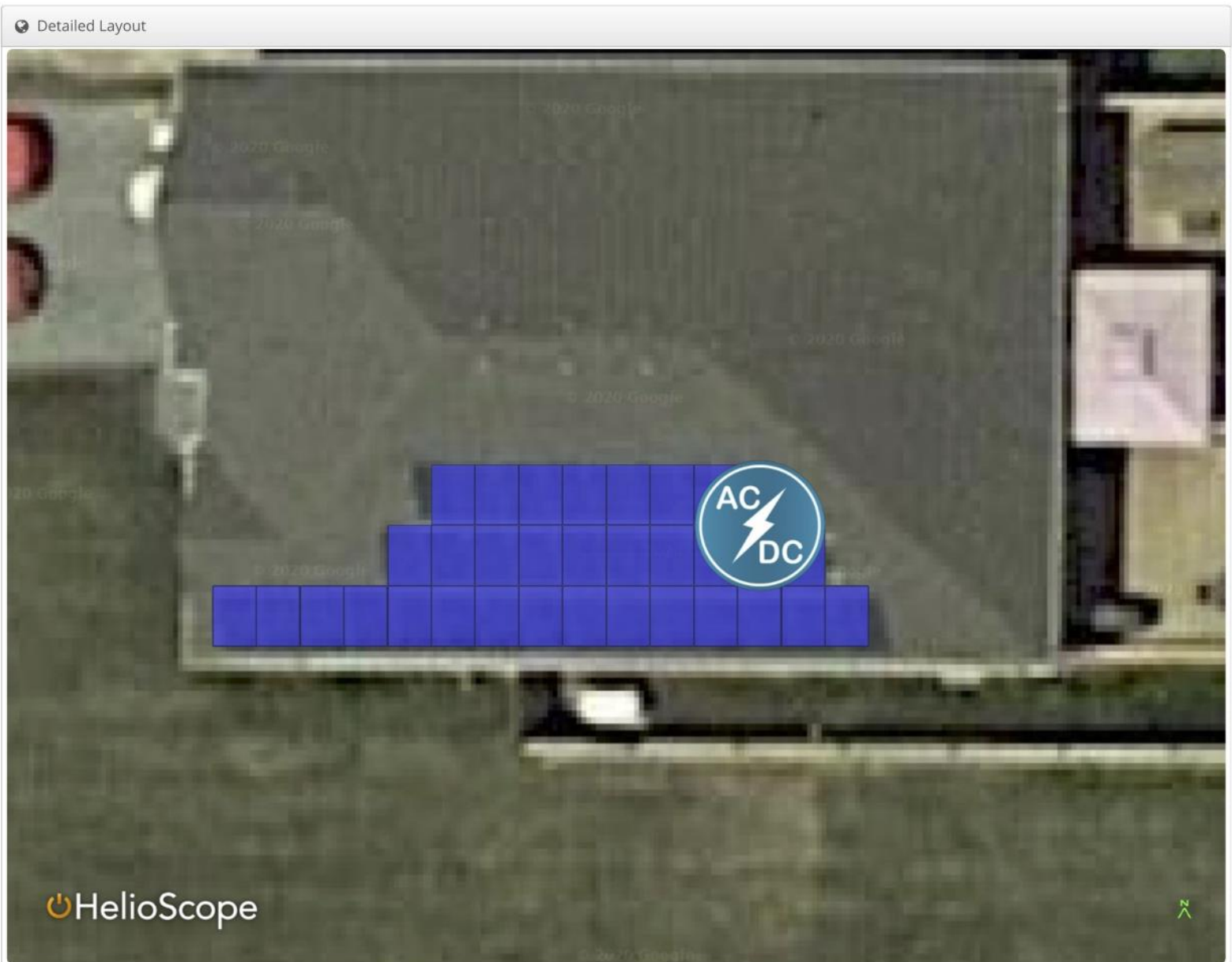




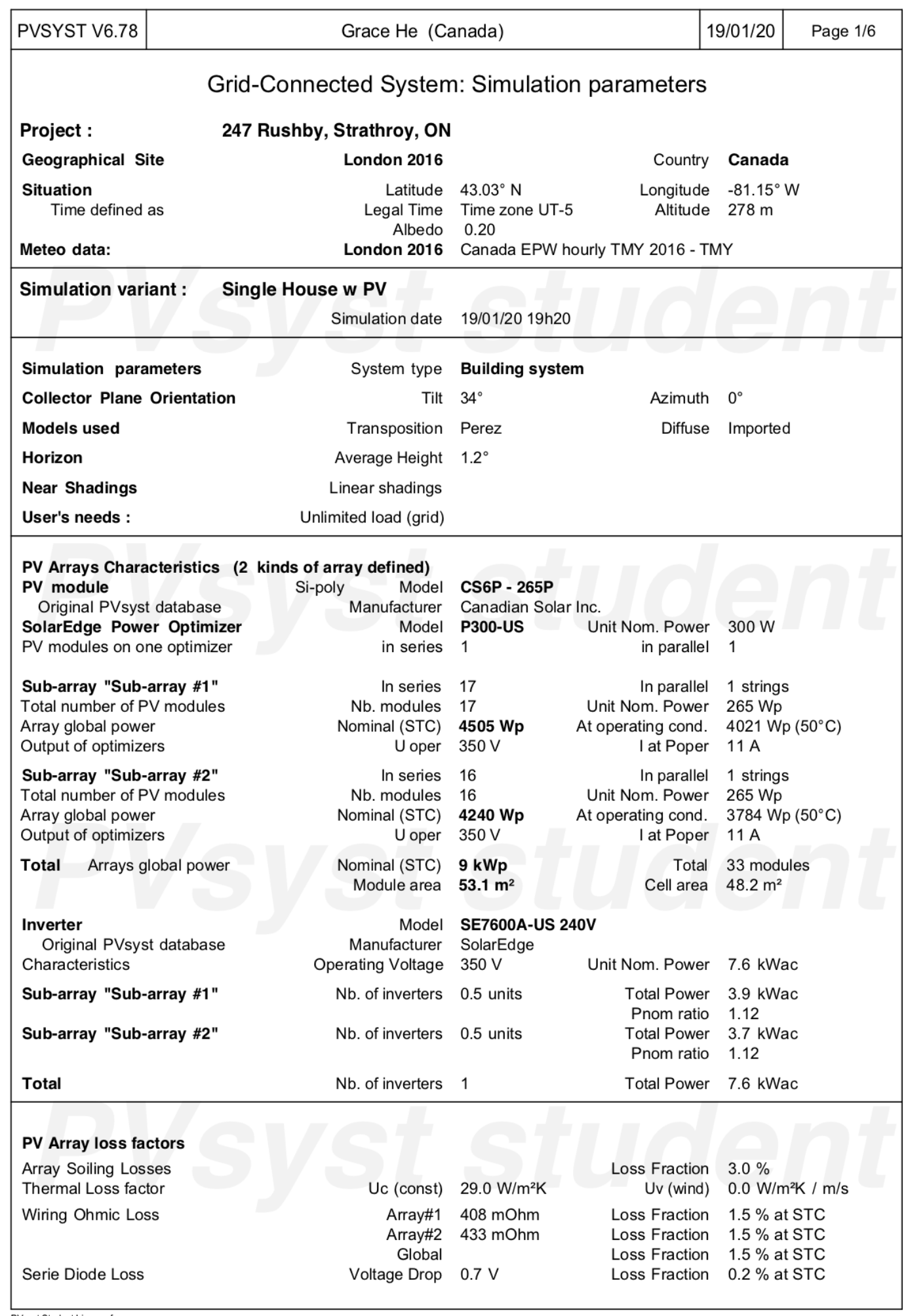




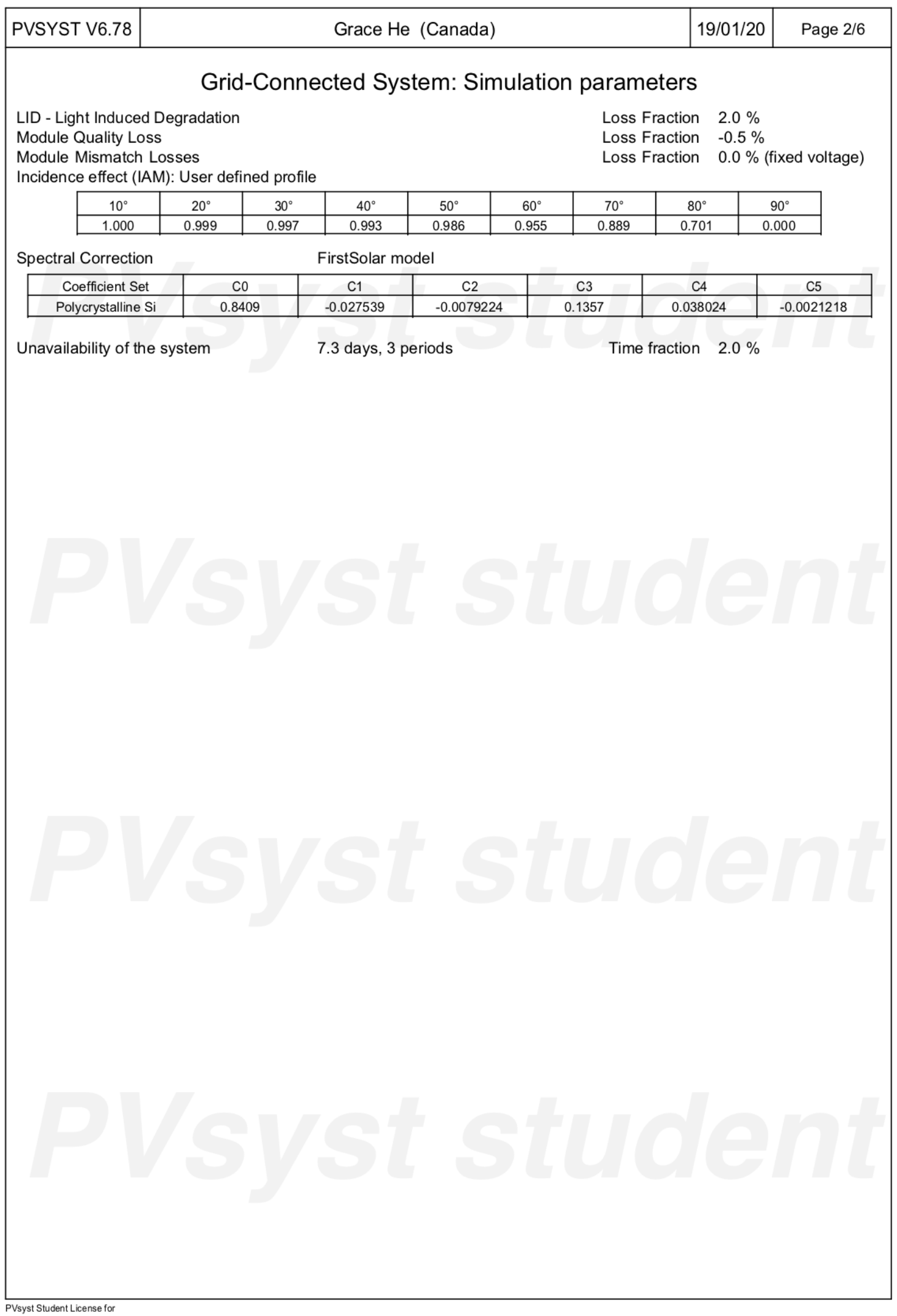




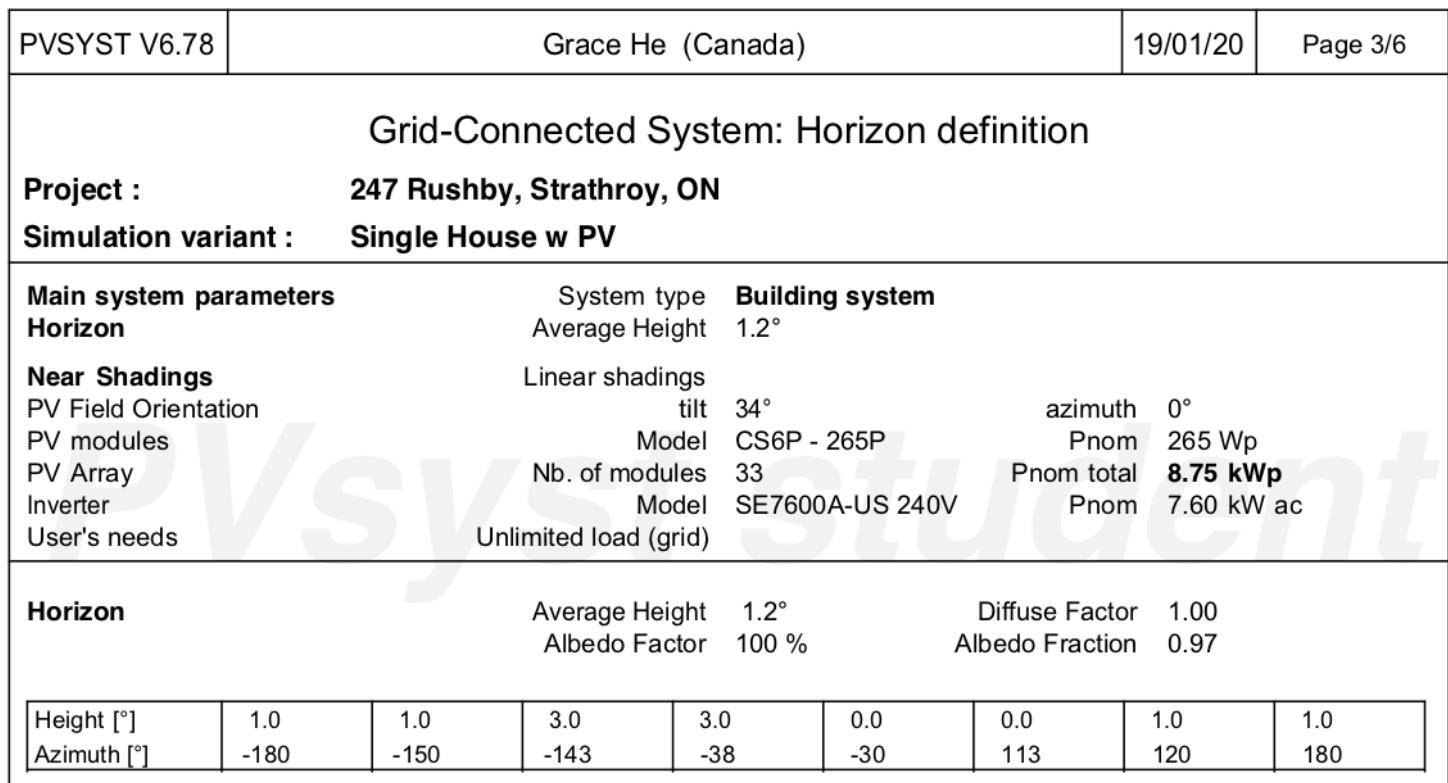

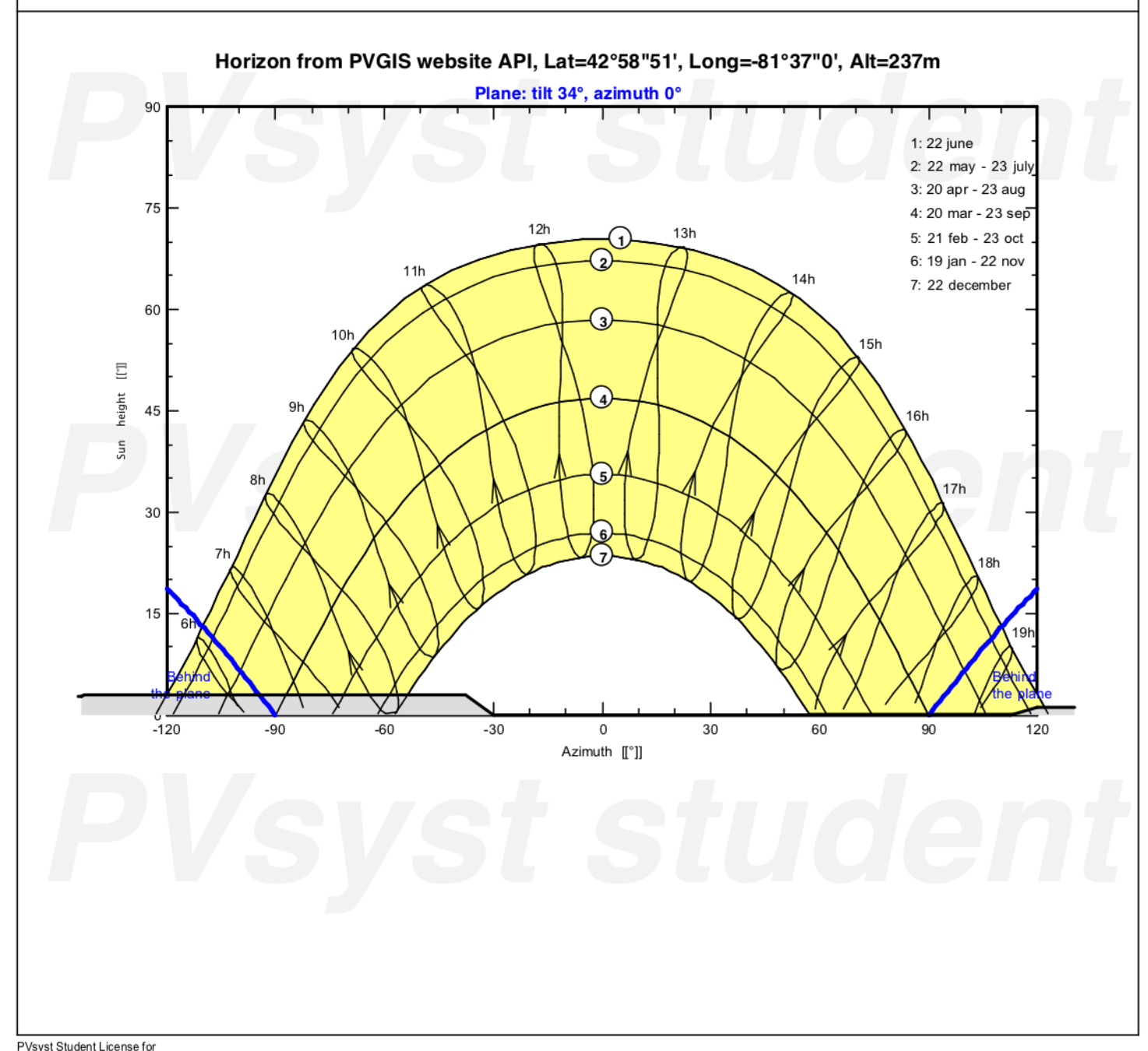




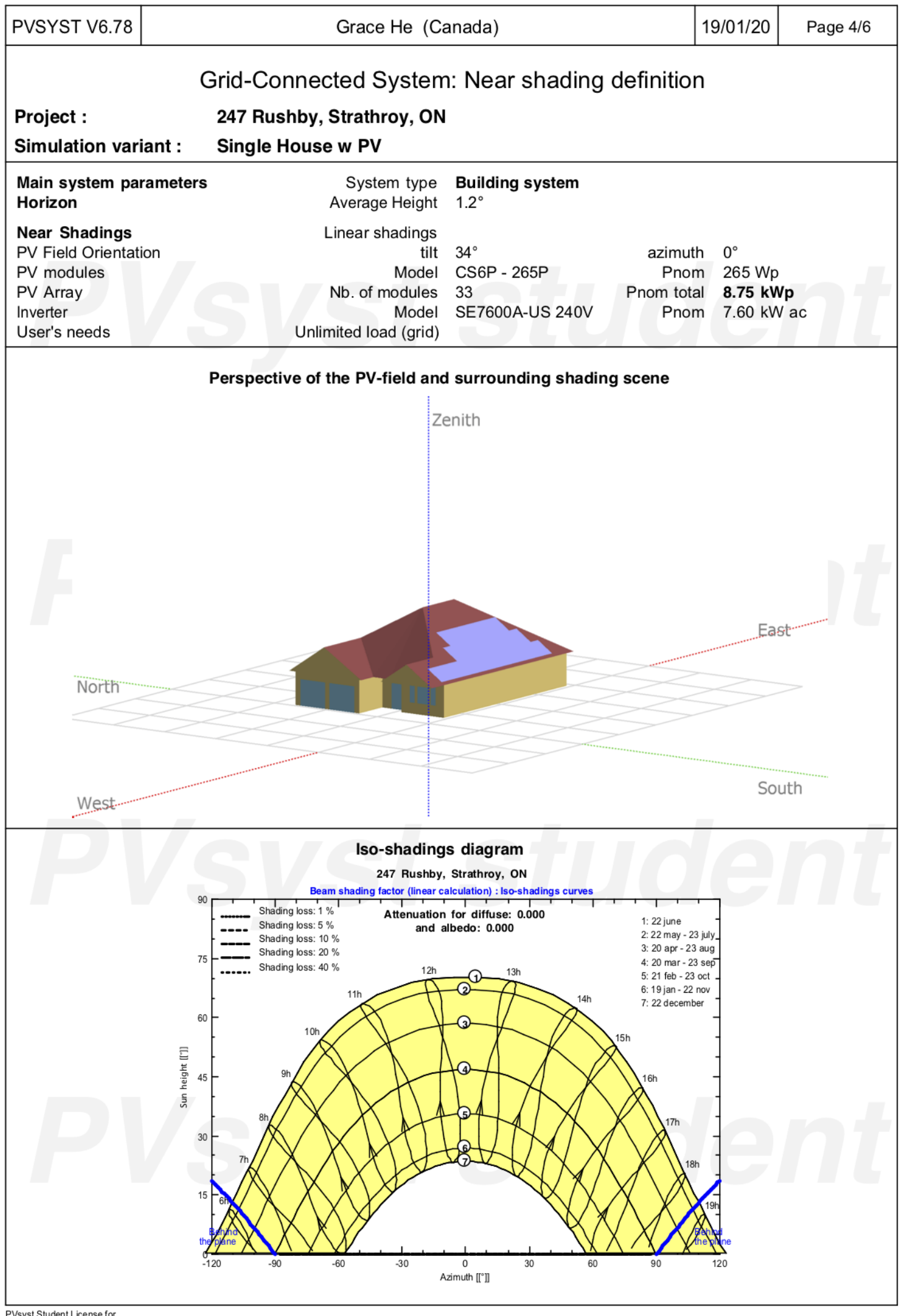




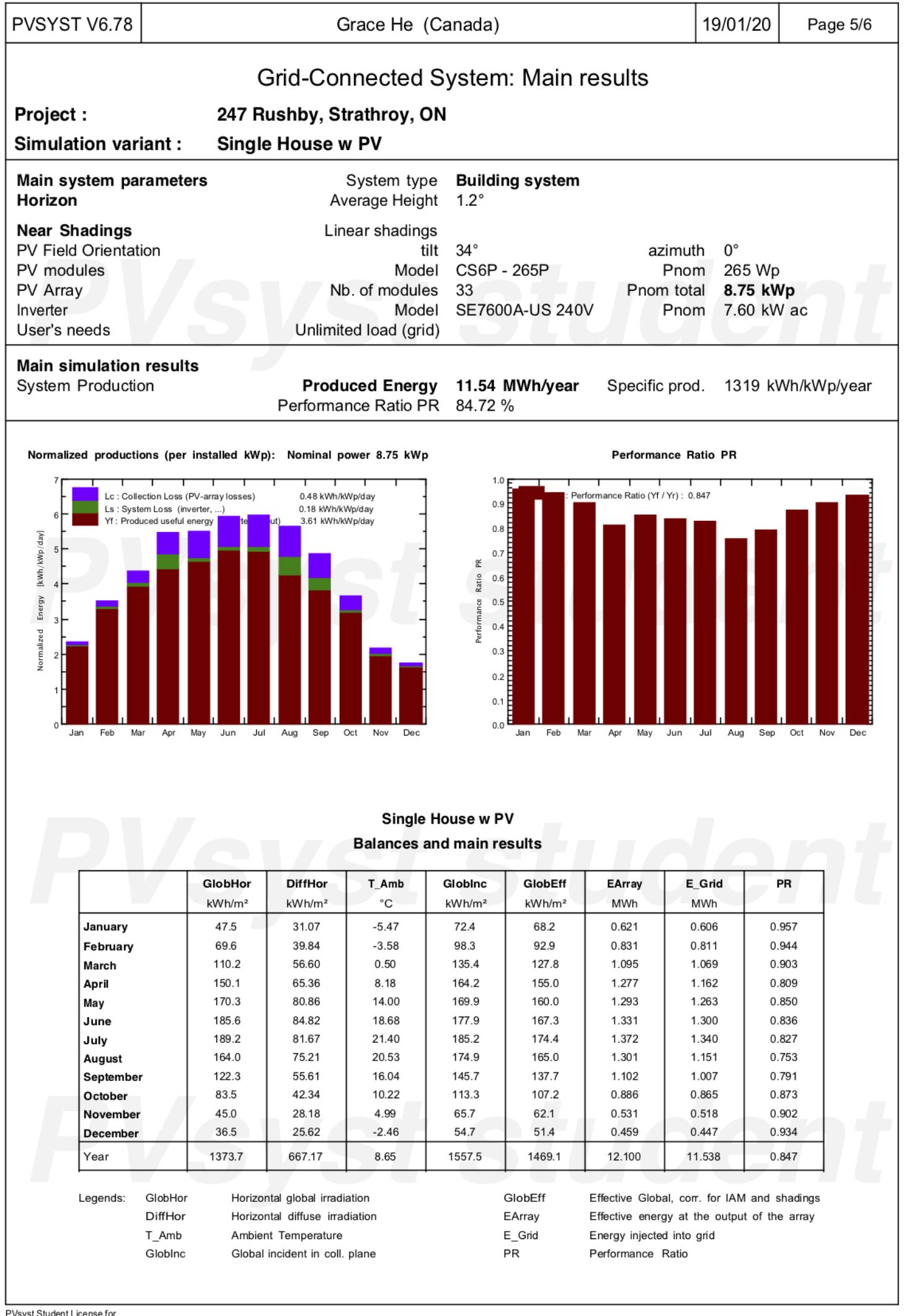




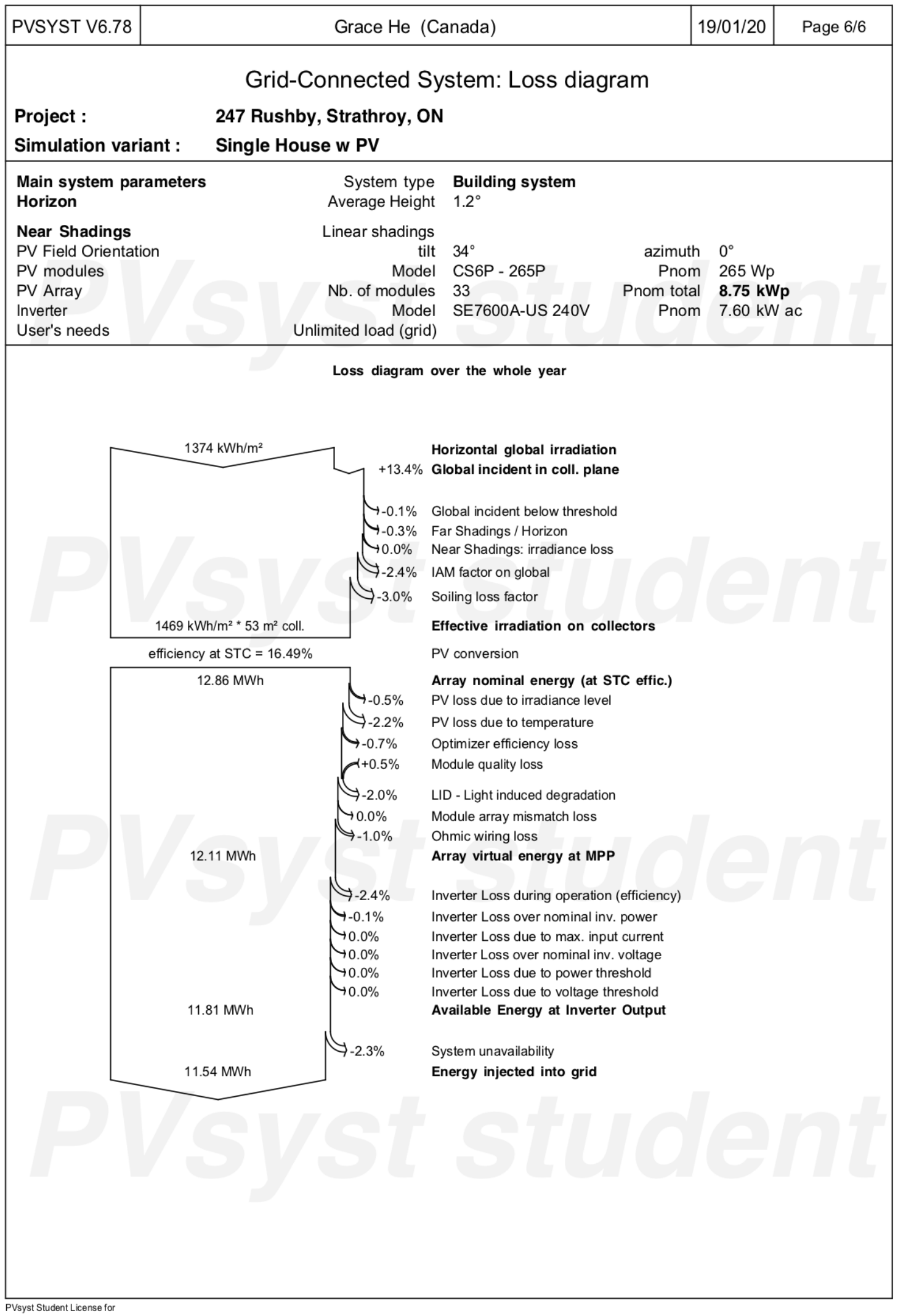


Appendix D. $P V * S O L$ Simulation Report

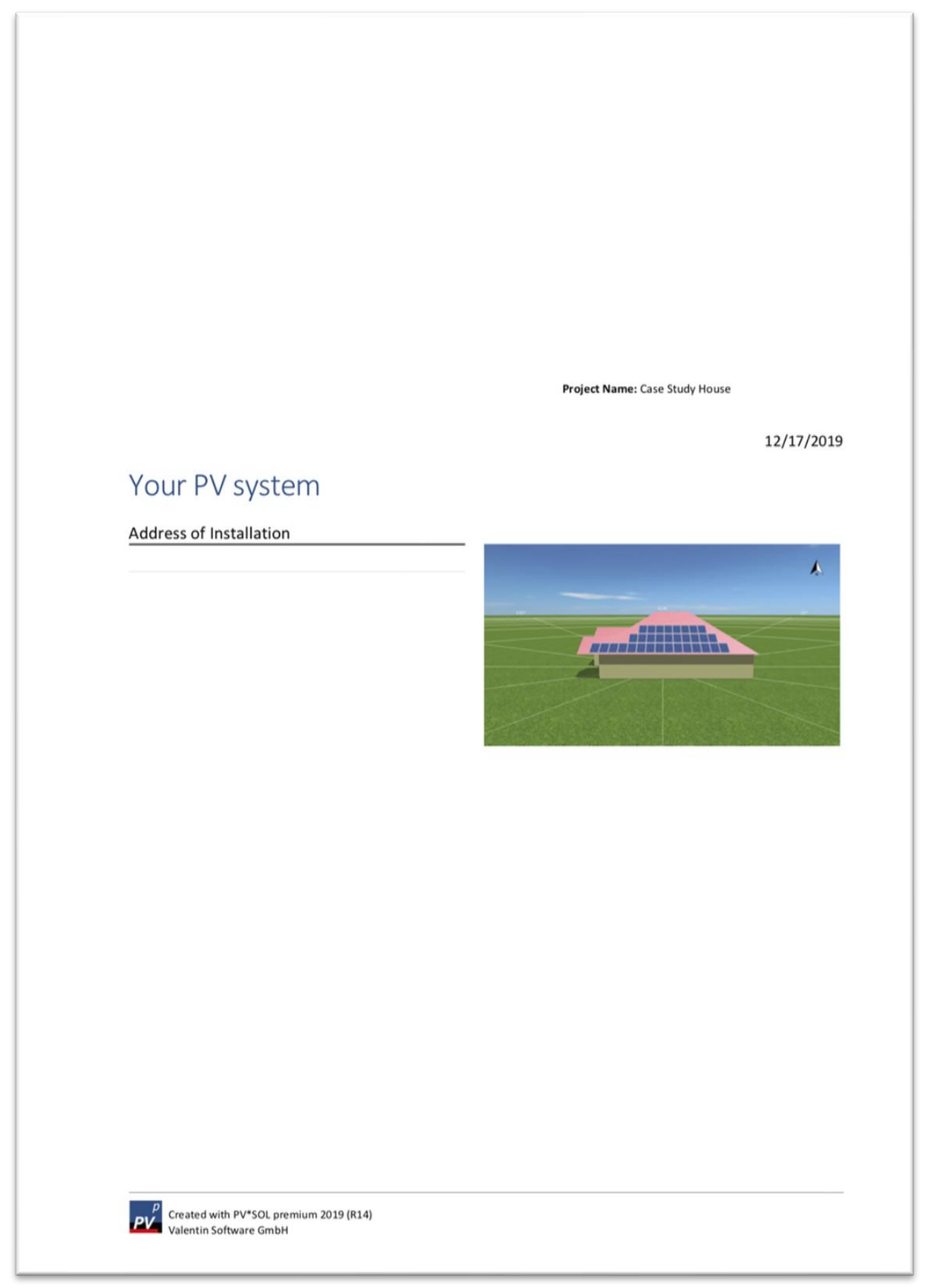




\section{Project Overview}

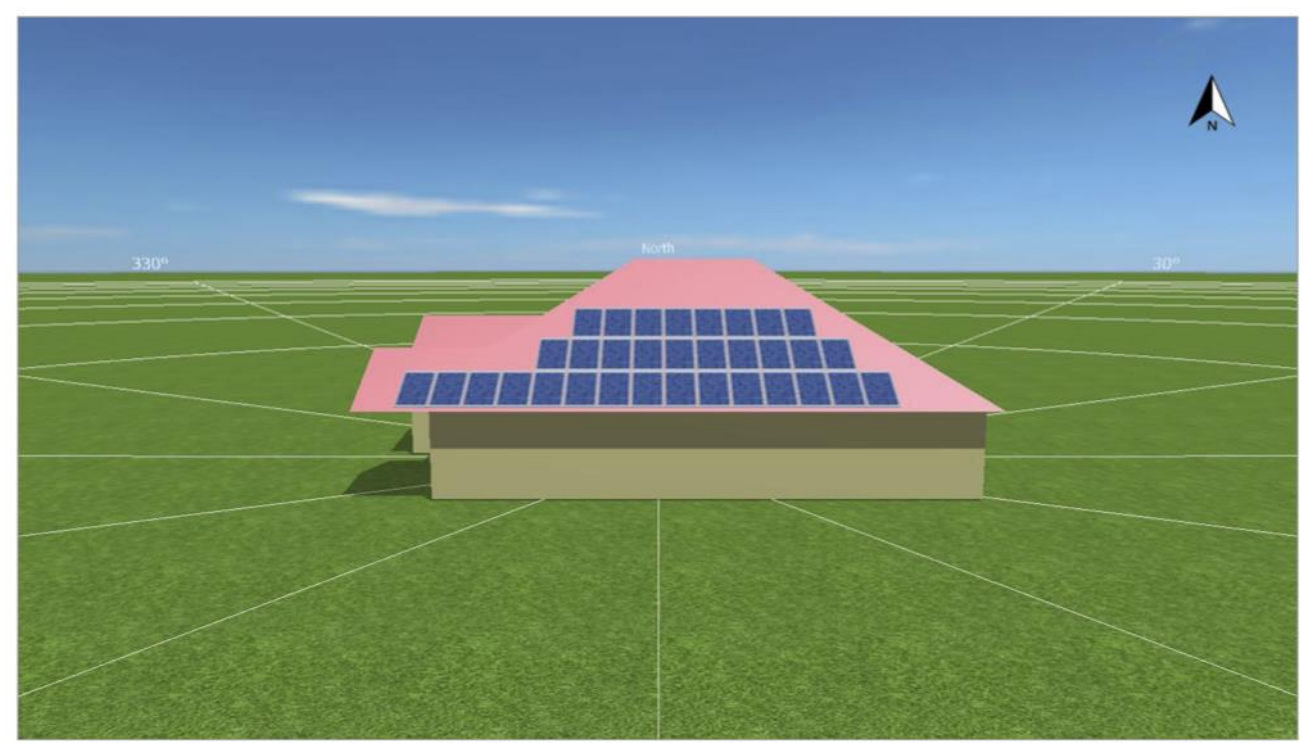

Figure: Overview Image, 3D Design

\section{PV System}

3D, Grid-connected PV System

Climate Data

London CWEC Converted 2016, CAN (-

)

PV Generator Output $8.75 \mathrm{kWp}$

PV Generator Surface $53.1 \mathrm{~m}^{2}$

Number of PV Modules 33

Number of Inverters 


\section{Case Study House}

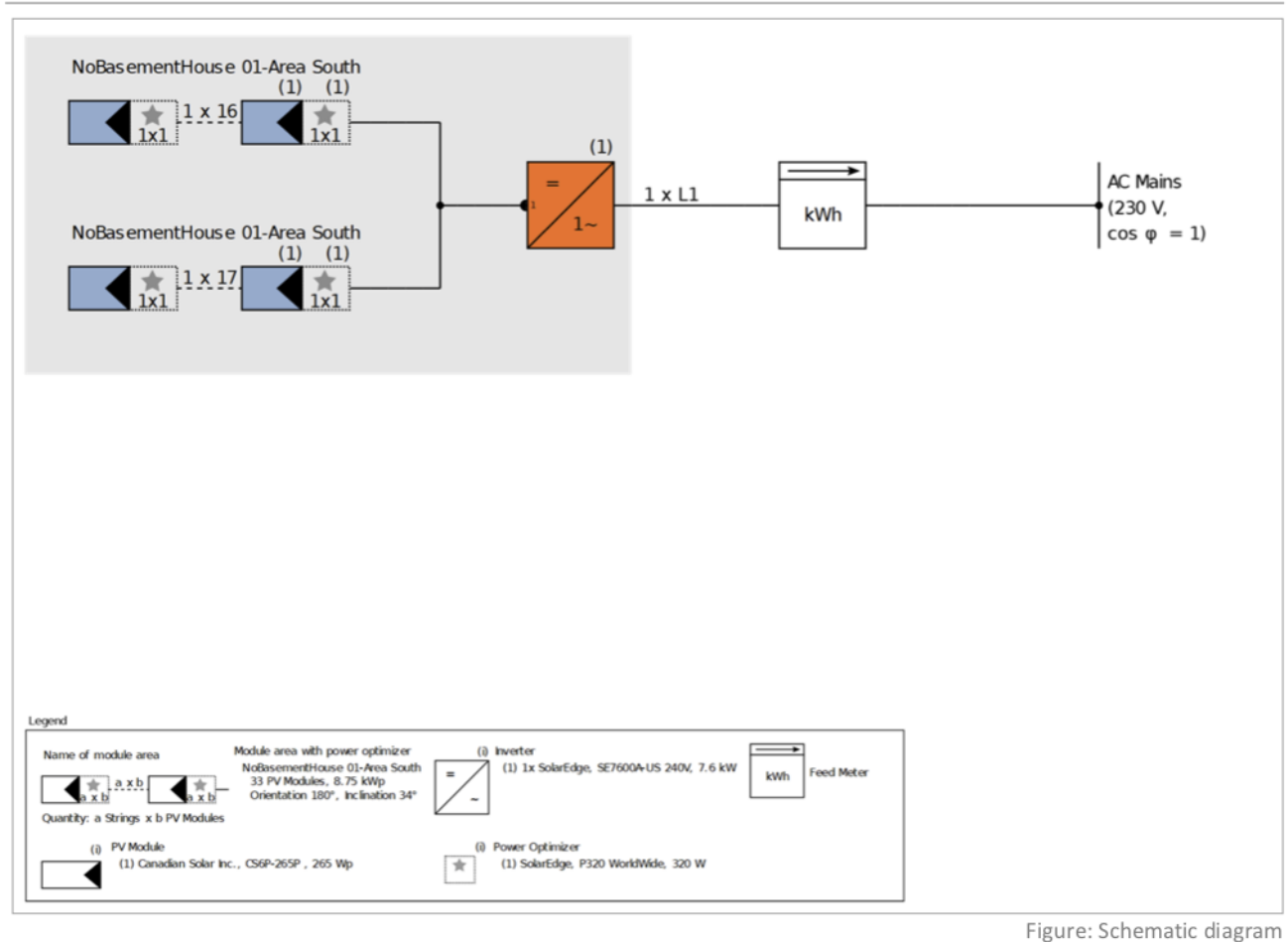

The yield

The yield

\begin{tabular}{lc}
\hline PV Generator Energy (AC grid) & $12,040 \mathrm{kWh}$ \\
\hline Grid Feed-in & $12,040 \mathrm{kWh}$ \\
\hline Down-regulation at Feed-in Point & $0 \mathrm{kWh}$ \\
\hline Own Power Consumption & $0.0 \%$ \\
Solar Fraction & $0.0 \%$ \\
\hline Spec. Annual Yield & $1,376.76 \mathrm{kWh} / \mathrm{kWp}$ \\
Performance Ratio (PR) & $90.9 \%$ \\
\hline Yield Reduction due to Shading & $0.1 \% /$ year \\
$\mathrm{CO}_{2}$ Emissions avoided & $7,224 \mathrm{~kg} /$ year
\end{tabular}

Financial Analysis

Your Gain

Total investment costs

Return on Assets

$13,117.50 \$$

Amortization Period

$9.44 \%$

Electricity Production Costs

9.3 Years

Energy Balance/Feed-in Concep

$0.06 \$ / k W h$

Full Feed-in

The results have been calculated with a mathematical model calculation from Valentin Software GmbH ( $\mathrm{PV}$ *SOL algorithms). The actual yields from the solar power system may differ as a result of weather variations, the efficiency of the modules and inverter and other factors. 


\section{Case Study House}

\section{Set-up of the System}

\section{Overview}

\section{System Data}

Type of System

3D, Grid-connected PV System

Start of Operation

\section{Climate Data}

Location London CWEC Converted 2016, CAN ( - )

Resolution of the data

Simulation model used:

- Diffuse Irradiation onto Horizontal Plane

Hofmann

- Irradiance onto tilted surface

\section{Module Areas}

1. Module Area - NoBasementHouse 01-Area South

PV Generator, 1. Module Area - NoBasementHouse 01-Area South

\begin{tabular}{lc}
\hline Name & NoBasementHouse 01-Area South \\
PV Modules & $33 \times$ CS6P-265P \\
Manufacturer & Canadian Solar Inc. \\
Inclination & $34^{\circ}$ \\
Orientation & South $180^{\circ}$ \\
Installation Type & Mounted - Roof \\
PV Generator Surface & $53.1 \mathrm{~m}^{2}$
\end{tabular}

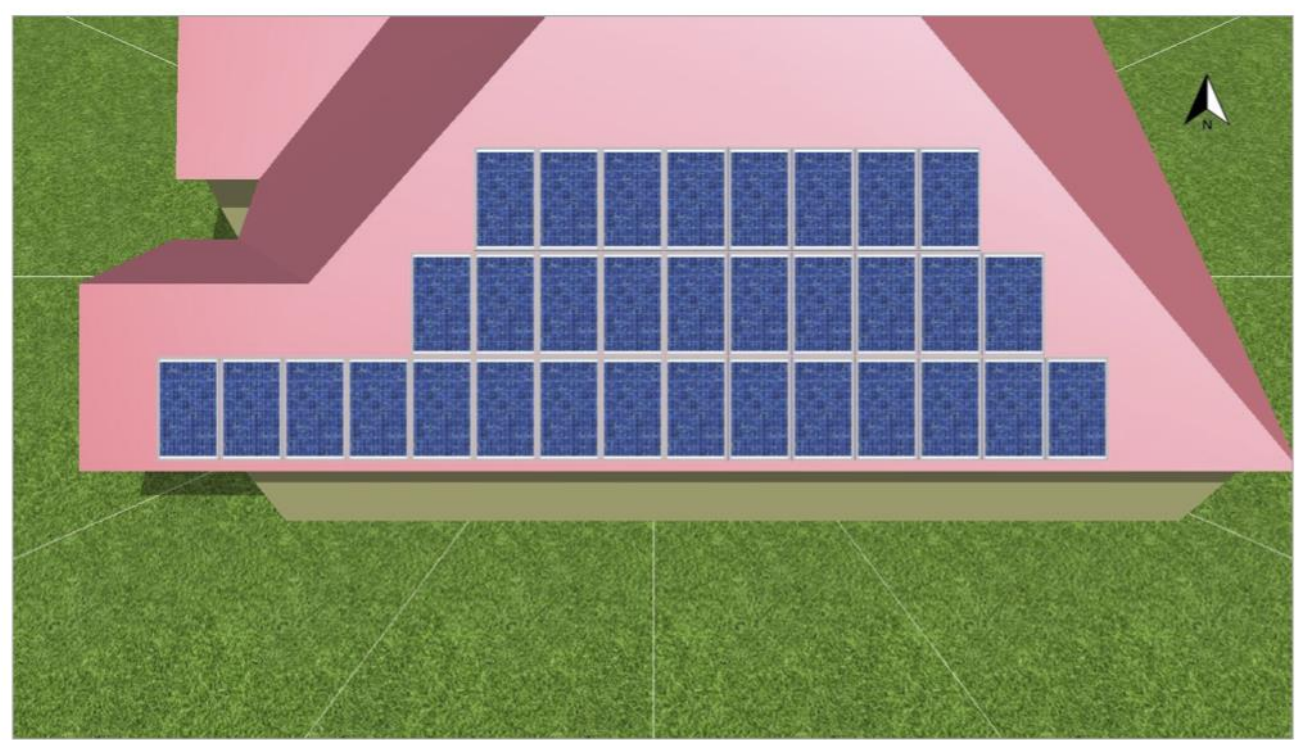

Figure: 1. Module Area - NoBasementHouse 01-Area South 


\section{Case Study House}

\section{Horizon Line, 3D Design}

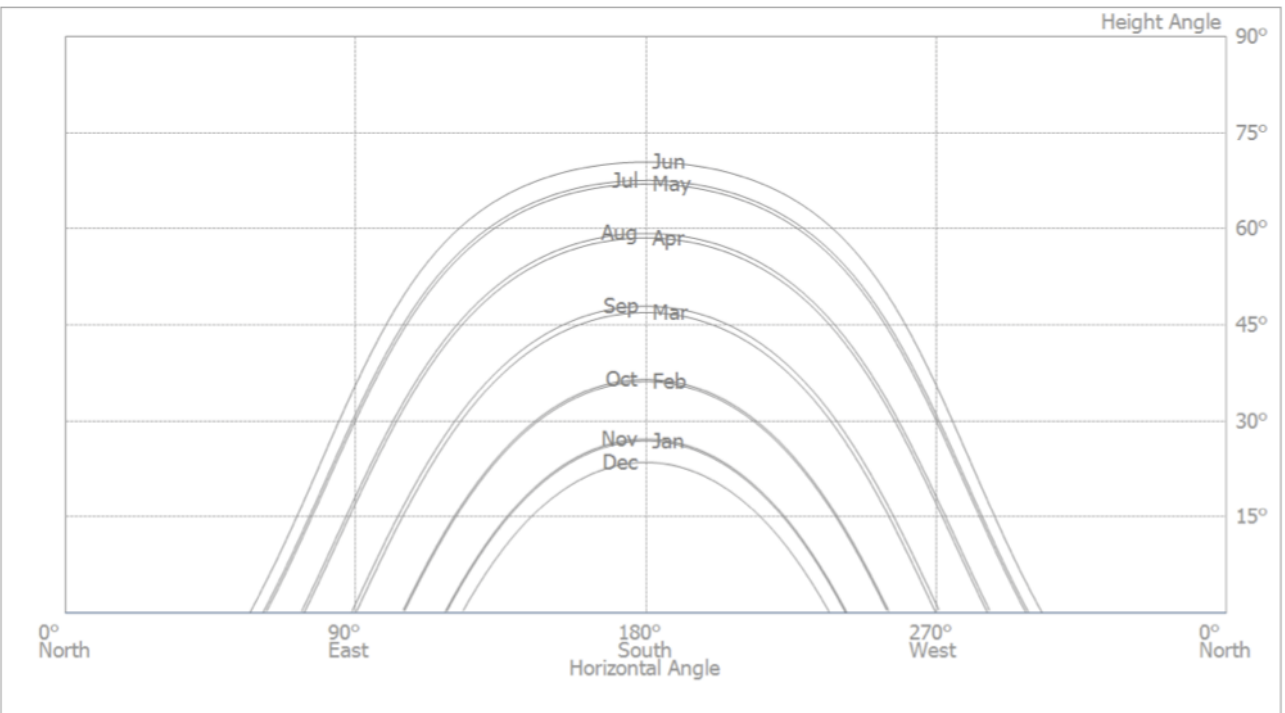

Figure: Horizon (3D Design)

\section{Inverter configuration}

\begin{tabular}{|c|c|}
\hline Module Area & NoBasementHouse 01-Area South \\
\hline \multicolumn{2}{|l|}{ Inverter 1} \\
\hline Manufacturer & SolarEdge \\
\hline Model & SE7600A-US 240V \\
\hline Quantity & 1 \\
\hline Sizing Factor & $115.1 \%$ \\
\hline \multirow[t]{2}{*}{ Configuration } & MPP 1: \\
\hline & 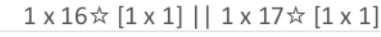 \\
\hline \multicolumn{2}{|c|}{ Power Optimizer 1} \\
\hline Manufacturer & SolarEdge \\
\hline Model & P320 WorldWide \\
\hline Quantity & 33 \\
\hline
\end{tabular}

AC Mains

AC Mains

Number of Phases

Mains Voltage (1-phase)

3

Displacement Power Factor (cos phi)

$230 \mathrm{~V}$

$+/-1$ 
Case Study House

\section{Simulation Results}

\section{Results Total System}

\section{PV System}

PV Generator Output

Spec. Annual Yield

Performance Ratio (PR)

$8.7 \mathrm{kWp}$

1,376.76 kWh/kWp

$90.9 \%$

Grid Feed-in

Grid Feed-in in the first year (incl. module degradation)

$0.1 \% /$ year

Standby Consumption (Inverter)

$\mathrm{CO}_{2}$ Emissions avoided

$12,040 \mathrm{kWh} /$ year $12,040 \mathrm{kWh} /$ year

$11 \mathrm{kWh} /$ year

$7,224 \mathrm{~kg} /$ year

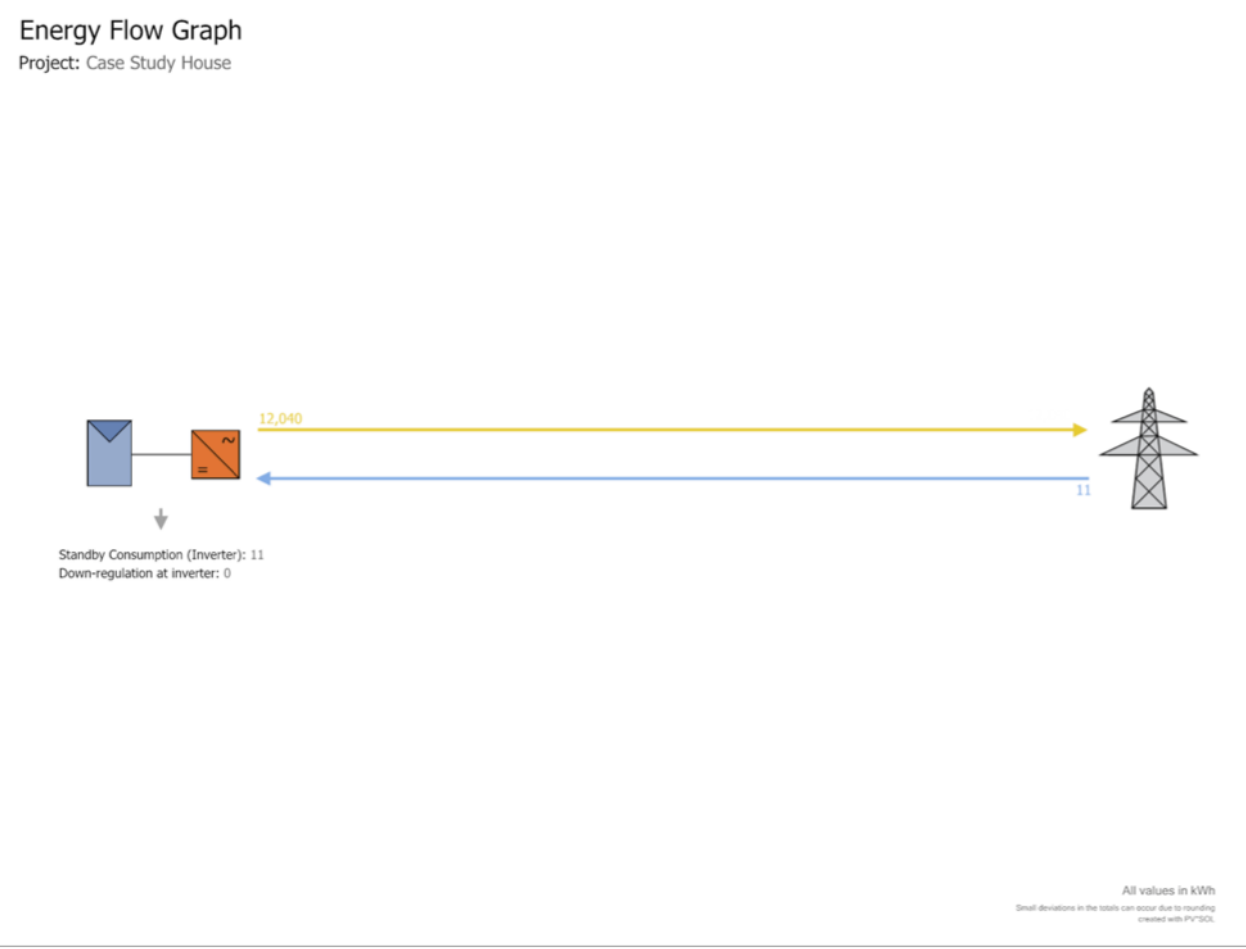

Figure: Energy Flow Graph 
Case Study House

\section{Financial Analysis}

\section{Overview}

\section{System Data}

Grid Feed-in in the first year (incl. module degradation)

PV Generator Output

Start of Operation of the System

$12,040 \mathrm{kWh} /$ year

Assessment Period

$10 / 22 / 2019$

Interest on Capital

20 Years

$1 \%$

Economic Parameters

\begin{tabular}{lc}
\hline Return on Assets & $9.44 \%$ \\
Accrued Cash Flow (Cash Balance) & $14,006.91 \$$ \\
Amortization Period & 9.3 Years \\
Electricity Production Costs & $0.06 \$ / \mathrm{kWh}$ \\
Payment Overview & \\
\hline Specific Investment Costs & $1,500.00 \$ / \mathrm{kWp}$ \\
Investment Costs & $13,117.50 \$$ \\
One-off Payments & $0.00 \$$ \\
Incoming Subsidies & $0.00 \$$ \\
Annual Costs & $0.00 \$ /$ year \\
Other Revenue or Savings & $0.00 \$ /$ year \\
\hline & \\
Remuneration and Savings & \\
\hline Total Payment from Utility in First Year & $1,496.54 \$ /$ year \\
\hline EEG 2015 (Mai) - Gebäudeanlage & \\
Validity & $10 / 23 / 2019-12 / 31 / 2039$ \\
\hline Specific feed-in / export Remuneration & $0.1243 \$ / \mathrm{kWh}$ \\
\hline Feed-in / Export Tariff & $1,496.54 \$ /$ year
\end{tabular}

Feed-in / Export Tariff

$1,496.54 \$ /$ year 


\section{Case Study House}

\section{Cash flow}

Cashflow Table

\begin{tabular}{|c|c|c|c|c|c|}
\hline & year 1 & year 2 & year 3 & year 4 & year 5 \\
\hline Investments & $(\$ 13,117.50)$ & $\$ 0.00$ & $\$ 0.00$ & $\$ 0.00$ & $\$ 0.00$ \\
\hline Feed-in / Export Tariff & $\$ 1,406.53$ & $\$ 1,467.06$ & $\$ 1,452.53$ & $\$ 1,438.15$ & $\$ 1,423.91$ \\
\hline Annual Cash Flow & $(\$ 11,710.97)$ & $\$ 1,467.06$ & $\$ 1,452.53$ & $\$ 1,438.15$ & $\$ 1,423.91$ \\
\hline \multirow[t]{2}{*}{$\begin{array}{l}\text { Accrued Cash Flow (Cash } \\
\text { Balance) }\end{array}$} & $(\$ 11,710.97)$ & $(\$ 10,243.91)$ & $(\$ 8,791.38)$ & $(\$ 7,353.23)$ & $(\$ 5,929.32)$ \\
\hline & year 6 & year 7 & year 8 & year 9 & year 10 \\
\hline Investments & $\$ 0.00$ & $\$ 0.00$ & $\$ 0.00$ & $\$ 0.00$ & $\$ 0.00$ \\
\hline Feed-in / Export Tariff & $\$ 1,409.81$ & $\$ 1,395.85$ & $\$ 1,382.03$ & $\$ 1,368.35$ & $\$ 1,354.80$ \\
\hline Annual Cash Flow & $\$ 1,409.81$ & $\$ 1,395.85$ & $\$ 1,382.03$ & $\$ 1,368.35$ & $\$ 1,354.80$ \\
\hline \multirow[t]{2}{*}{$\begin{array}{l}\text { Accrued Cash Flow (Cash } \\
\text { Balance) }\end{array}$} & $(\$ 4,519.51)$ & $(\$ 3,123.65)$ & $(\$ 1,741.62)$ & $(\$ 373.27)$ & $\$ 981.53$ \\
\hline & year 11 & year 12 & year 13 & year 14 & year 15 \\
\hline Investments & $\$ 0.00$ & $\$ 0.00$ & $\$ 0.00$ & $\$ 0.00$ & $\$ 0.00$ \\
\hline Feed-in / Export Tariff & $\$ 1,341.39$ & $\$ 1,328.11$ & $\$ 1,314.96$ & $\$ 1,301.94$ & $\$ 1,289.05$ \\
\hline Annual Cash Flow & $\$ 1,341.39$ & $\$ 1,328.11$ & $\$ 1,314.96$ & $\$ 1,301.94$ & $\$ 1,289.05$ \\
\hline \multirow[t]{2}{*}{$\begin{array}{l}\text { Accrued Cash Flow (Cash } \\
\text { Balance) }\end{array}$} & $\$ 2,322.92$ & $\$ 3,651.03$ & $\$ 4,965.98$ & $\$ 6,267.92$ & $\$ 7,556.97$ \\
\hline & year 16 & year 17 & year 18 & year 19 & year 20 \\
\hline Investments & $\$ 0.00$ & $\$ 0.00$ & $\$ 0.00$ & $\$ 0.00$ & $\$ 0.00$ \\
\hline Feed-in / Export Tariff & $\$ 1,276.28$ & $\$ 1,263.65$ & $\$ 1,251.14$ & $\$ 1,238.75$ & $\$ 1,226.48$ \\
\hline Annual Cash Flow & $\$ 1,276.28$ & $\$ 1,263.65$ & $\$ 1,251.14$ & $\$ 1,238.75$ & $\$ 1,226.48$ \\
\hline \multirow[t]{2}{*}{$\begin{array}{l}\text { Accrued Cash Flow (Cash } \\
\text { Balance) }\end{array}$} & $\$ 8,833.25$ & $\$ 10,096.90$ & $\$ 11,348.04$ & $\$ 12,586.79$ & $\$ 13,813.27$ \\
\hline & year 21 & & & & \\
\hline Investments & $\$ 0.00$ & & & & \\
\hline Feed-in / Export Tariff & $\$ 193.64$ & & & & \\
\hline Annual Cash Flow & $\$ 193.64$ & & & & \\
\hline $\begin{array}{l}\text { Accrued Cash Flow (Cash } \\
\text { Balance) }\end{array}$ & $\$ 14,006.91$ & & & & \\
\hline
\end{tabular}


Case Study House

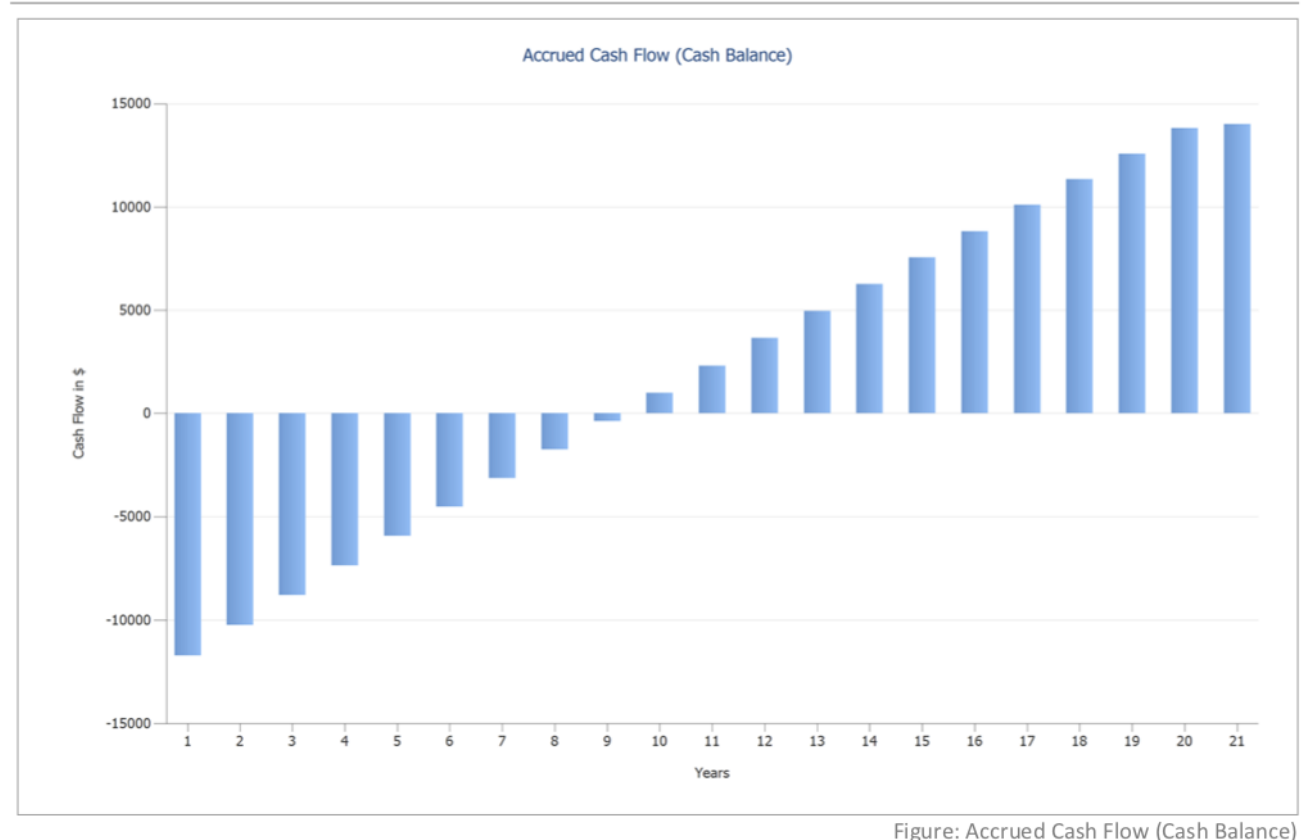

PV Created with PV*SOL premium 2019 (R14)

PV Valentin Software GmbH

Page 9 of 12 
Case Study House

Plans

Circuit Diagram

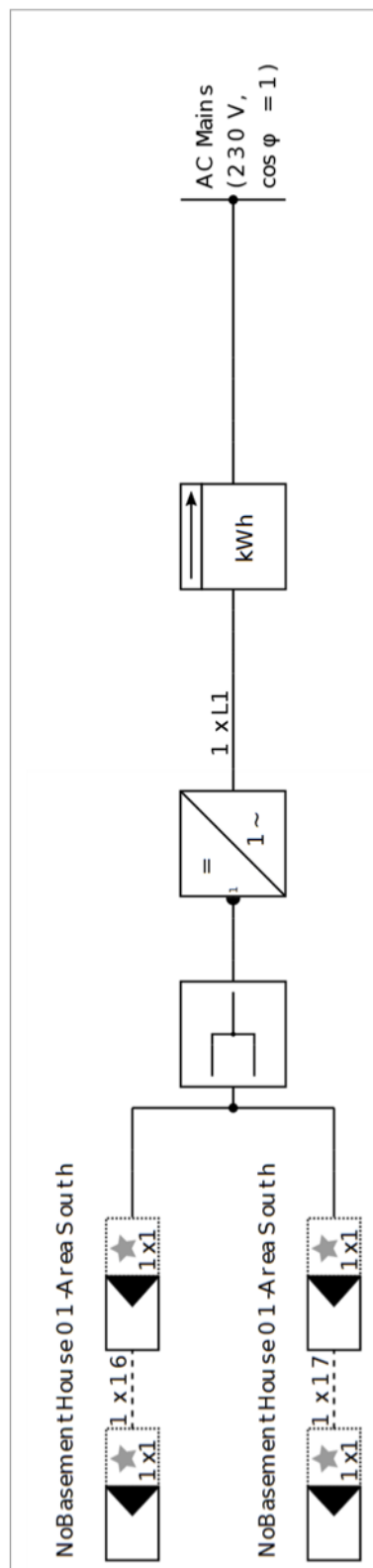

Figure: Circuit Diagram 
Case Study House

\section{Dimensioning Plan}

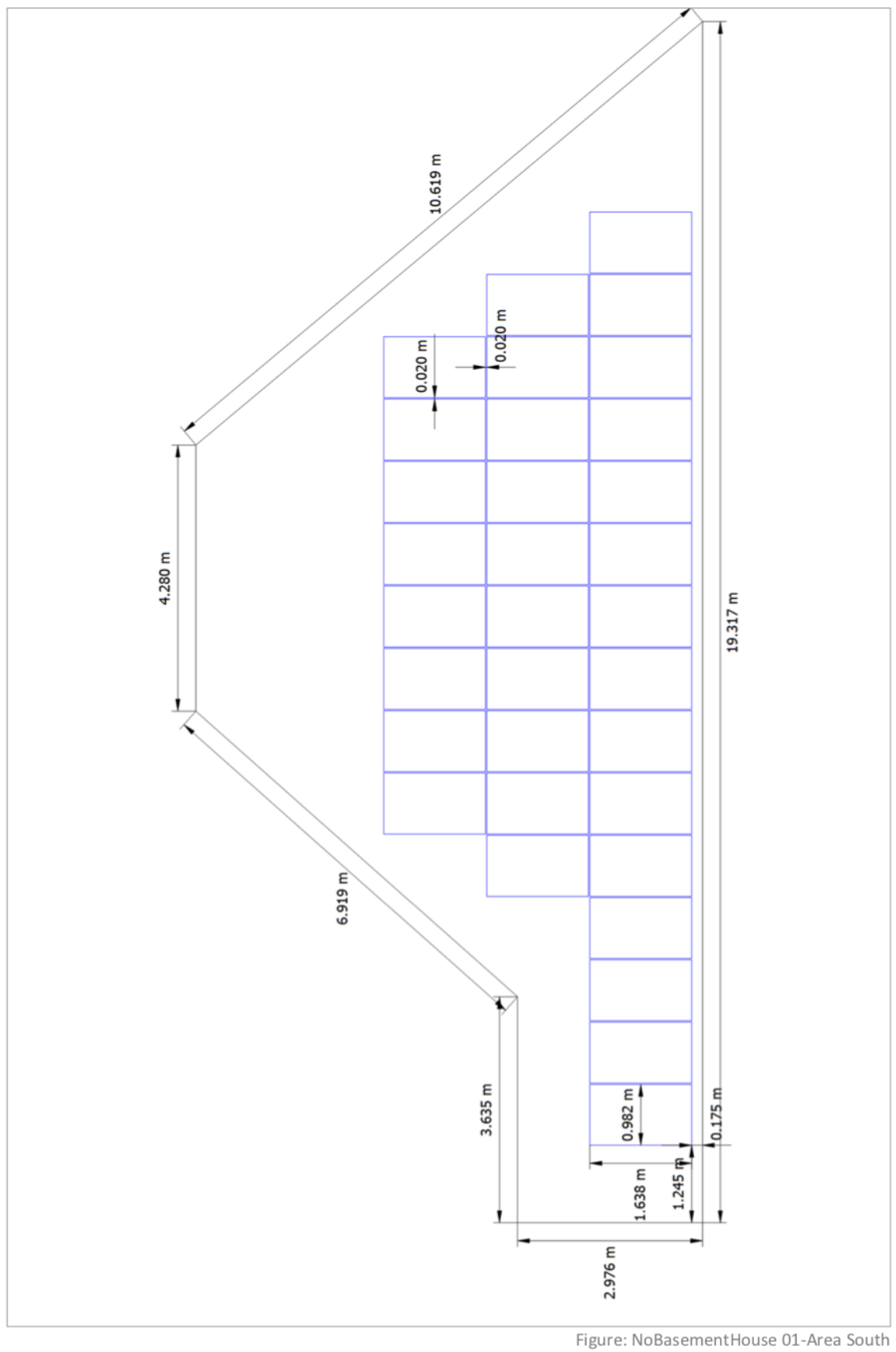


Case Study House

String Plan

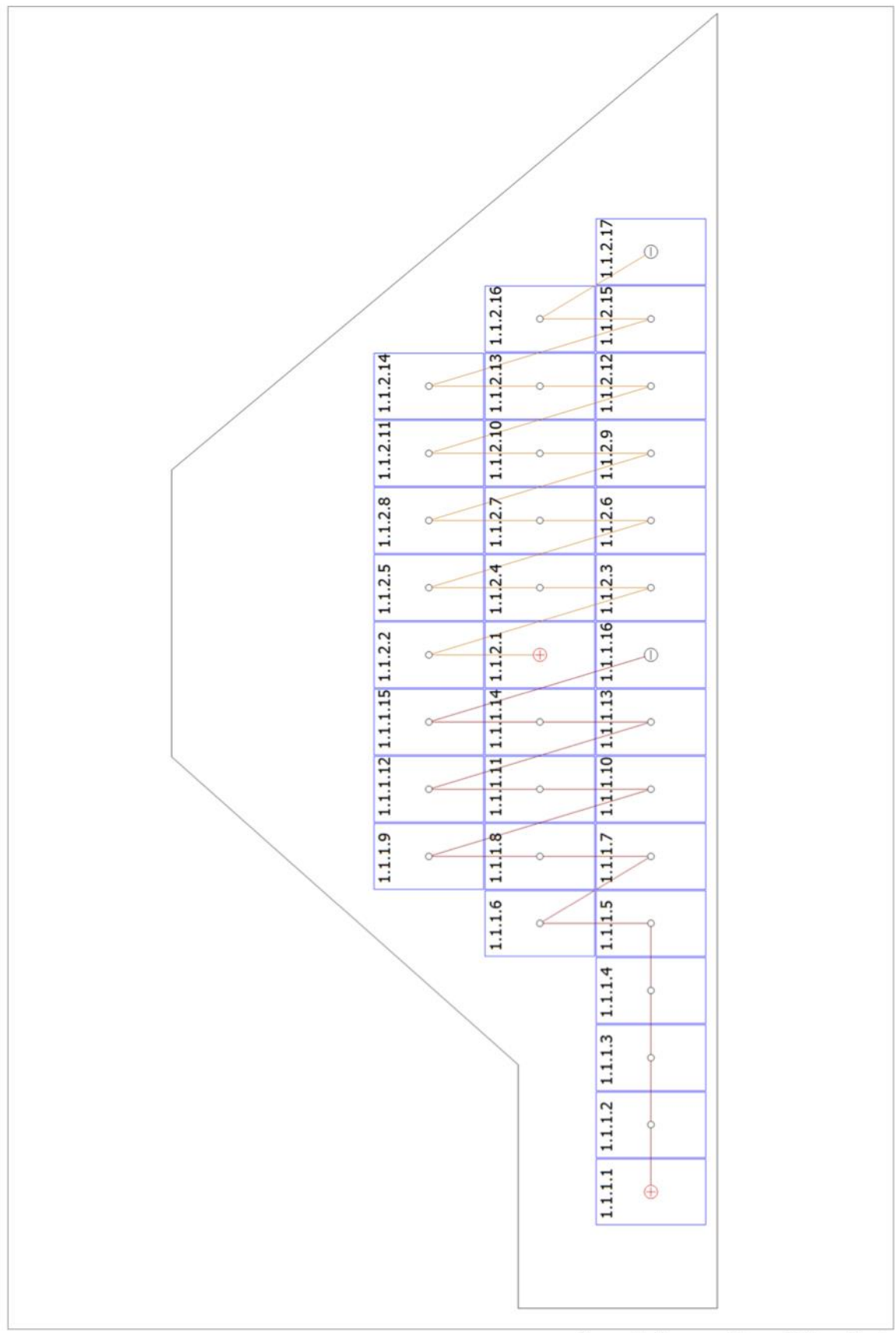

Figure: NoBasementHouse 01-Area South 
Appendix E. Archelios Simulation Report

archelios PRO archelios Pro study report (Version: 2020.1.01)

\section{Photovoltaic system sizing report}

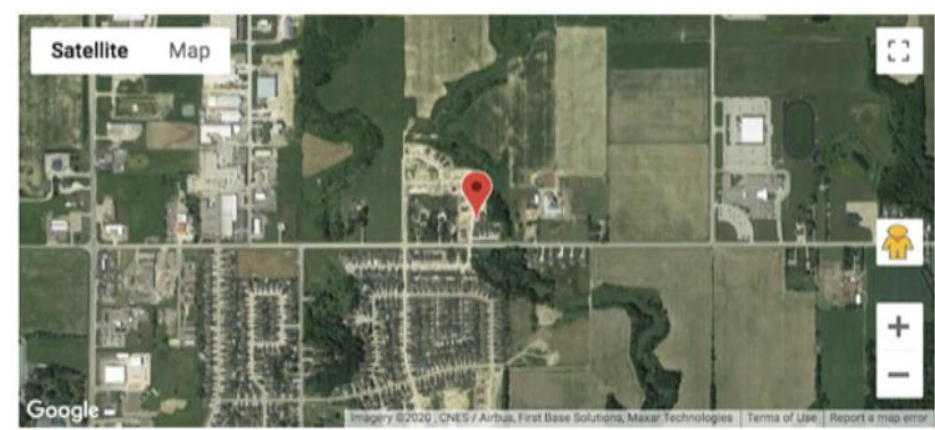

Project name: Archelios Jan0820

Address: 


\section{archelios PRO archelios Pro study report (Version: 2020.1.01)}

四 Reference meteo station

Reference station: London Airp. ON Altitude: $278 \mathrm{~m}$

Distance to project: $38.40 \mathrm{~km}$

Irradiation:

Global irradiation: 1,354.0 kWh/m2.year

Direct irradiation: $701.0 \mathrm{kWh} / \mathrm{m} 2$.year

Diffuse irradiation: $653.0 \mathrm{kWh} / \mathrm{m} 2$.year

Data source: Meteonorm data (from archelios

database),Ta Température: 2000-2009, Ins.

Rayonnement: 1991-2010.

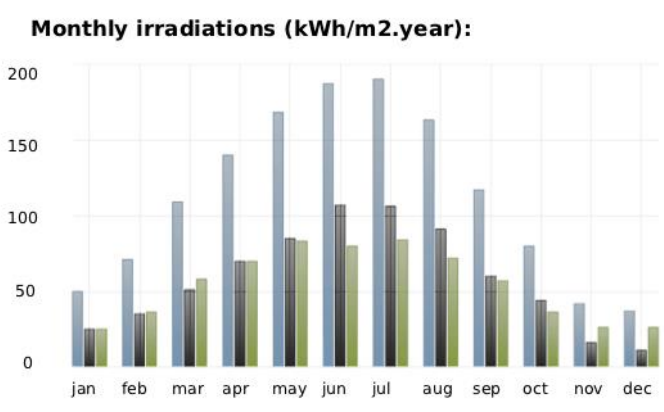

Solar fraction $(\%)$ :

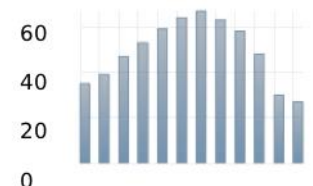

0

j f mamj j a sond
Wind speed $(\mathrm{m} / \mathrm{s})$ :

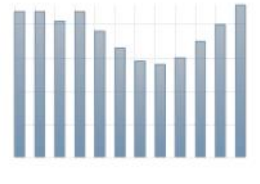

j f ma mj j a s ond
Air temperature $\left({ }^{\circ} \mathrm{C}\right)$ :

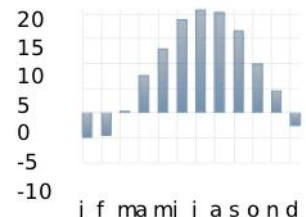

Linke trouble:

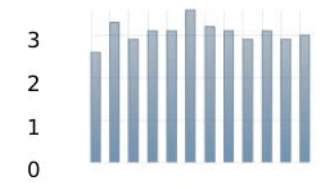

j f mamj j as ond

\section{四 Site}

Horizon line and monthly solar trajectories:

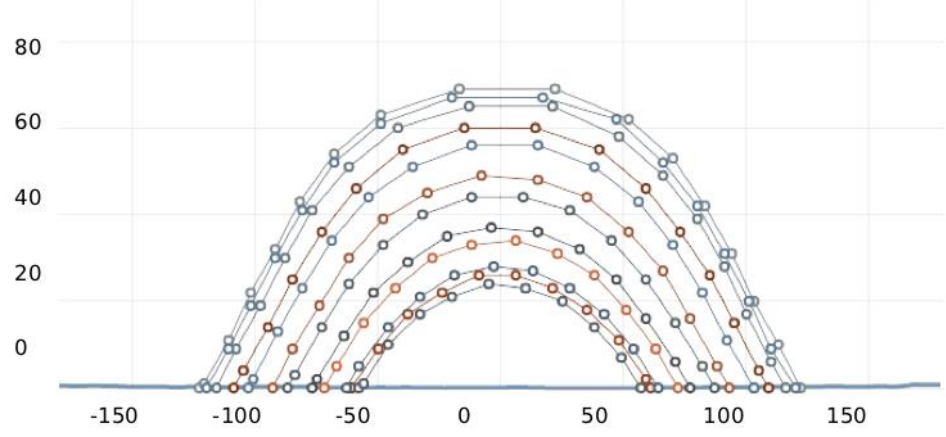

\section{Irradiation:}

Horizontal without shadings:

Global: 1,351.7 kWh/m2.year

Direct: $699.3 \mathrm{kWh} / \mathrm{m} 2$.year

Diffuse: $652.5 \mathrm{kWh} / \mathrm{m} 2$.year

\section{Horizontal with shadings:}

Global: 1,350.3 kWh/m2.year

Direct: $699.3 \mathrm{kWh} / \mathrm{m} 2$.year

Diffuse: $651.0 \mathrm{kWh} / \mathrm{m} 2$.year 
Simulation parameters

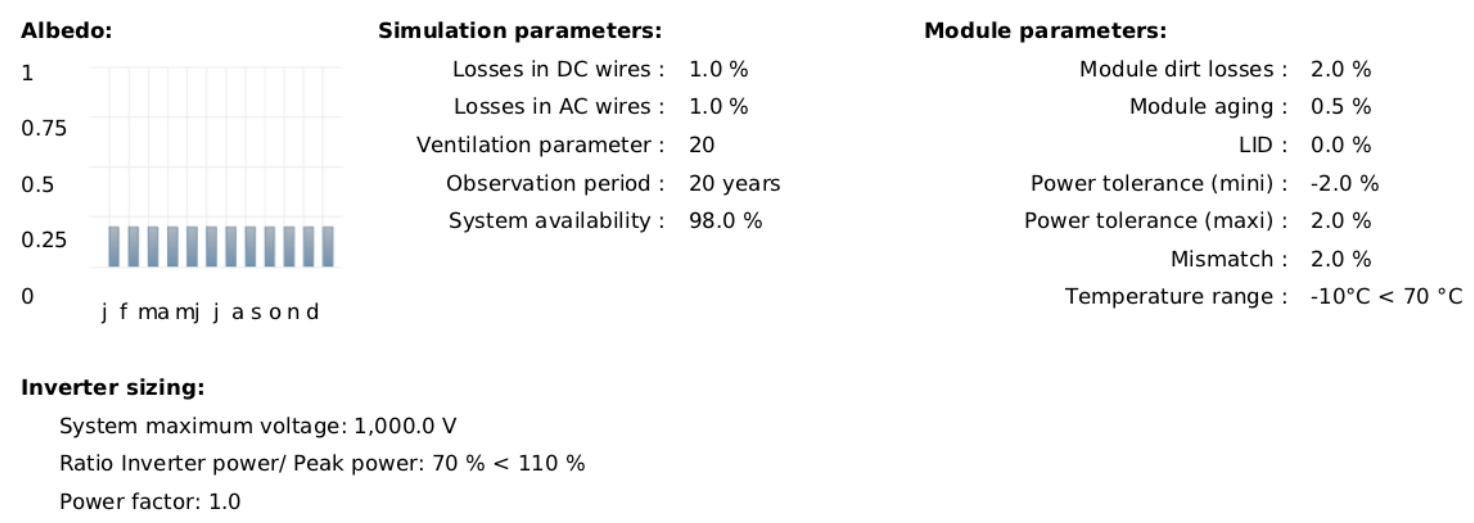

\section{囲PV array configurations}

Total power: $8.75 \mathrm{kWp}$

Total number of modules: 33

Total number of inverters: 3

\begin{tabular}{|c|c|c|c|c|c|c|c|c|}
\hline Inverter & Mppt & Module & Wp & $\mathrm{mod} / \mathrm{string}$ & string/ond & Wp total & Orientation & Tilt \\
\hline Inv ref 0 & 1 & Mod ref 0 & 265 & 11 & 1 & 2915 & 0 & 34 \\
\hline Inv ref 0 & 1 & Mod ref 0 & 265 & 11 & 1 & 2915 & 0 & 34 \\
\hline Inv ref 0 & 1 & Mod ref 0 & 265 & 11 & 1 & 2915 & 0 & 34 \\
\hline Inverter & Mppt & Module & \multicolumn{2}{|c|}{ Validity } & \multicolumn{2}{|c|}{ Ratio Pinv/Ppeak (\%) } & & \\
\hline Inv ref 0 & 1 & Mod ref 0 & $\sqrt{\mathbf{v}}$ & OK & \multicolumn{2}{|c|}{102.92} & & \\
\hline Inv ref 0 & 1 & Mod ref 0 & 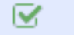 & OK & \multicolumn{2}{|c|}{102.92} & & \\
\hline Inv ref 0 & 1 & Mod ref 0 & $\underline{\nabla}$ & OK & \multicolumn{2}{|c|}{102.92} & & \\
\hline
\end{tabular}

\begin{tabular}{|c|c|c|c|c|c|}
\hline \multicolumn{4}{|c|}{$\begin{array}{l}\text { Characteristics - Inverter } \\
\text { Inv ref } 0 \text { : SolarEdge - SE3000 }\end{array}$} & \multirow{2}{*}{$\begin{array}{l}\text { Pstc (W) } \\
\text { Max eff. (\%) }\end{array}$} & \multirow{2}{*}{\begin{tabular}{|l|}
3000 \\
97.6
\end{tabular}} \\
\hline AC Pmax (W) & 3750 & $V \min (V)$ & 0 & & \\
\hline$I \max (\mathrm{A})$ & 11.5 & Vmax MPPT (V) & 500 & Euro eff. (\%) & 97.6 \\
\hline Number of entries & 1 & $V \max (\mathrm{V})$ & 500 & Protection type & IP 65 \\
\hline
\end{tabular}

Characteristics - PV module 


\section{archelíos PRO archelios Pro study report (Version: 2020.1.01)}

\begin{tabular}{|c|c|c|c|c|c|}
\hline \multicolumn{4}{|c|}{ Mod ref 0 : Canadian Solar Inc. - CS6P-265P } & Pstc (W) & \multirow{2}{*}{$\begin{array}{l}265 \\
45\end{array}$} \\
\hline Type & multicrystalline (mc-Si) & Uoc (V) & 37.7 & NOCT $\left({ }^{\circ} \mathrm{C}\right)$ & \\
\hline Total nb cells & 60 & Umpp (V) & 30.6 & Power coef. $\left(\% /{ }^{\circ} \mathrm{C}\right)$ & -0.41 \\
\hline Length $(\mathrm{mm})$ & 1638 & $\operatorname{Isc}(A)$ & 9.23 & Current coef. $\left(\% /{ }^{\circ} \mathrm{C}\right)$ & 0.053 \\
\hline Width (mm) & 982 & Impp (A) & 8.66 & Voltage coef. $\left(\% /{ }^{\circ} \mathrm{C}\right)$ & -0.31 \\
\hline
\end{tabular}




\section{archelios PRO archelios Pro study report (Version: 2020.1.01)}

C) 3D view

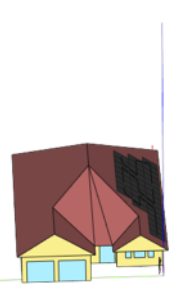


Production

Peak power: 8.75 kWp

Module surface: $53.1 \mathrm{~m}^{2}$

First year results:

Annual production (DC) : $\quad 12,170 \mathrm{kWh}$

Annual production (AC) :

AC specific yield (P50):

AC specific yield (P90) :

Performance ratio :

Mean values:

Annual production (DC) :

Annual production (AC) :

AC specific yield (P50) :

AC specific yield (P90) :

Performance ratio :
1,297 kWh/kWp

$1,190 \mathrm{kWh} / \mathrm{kWp}$

$81.65 \%$

$11,609 \mathrm{kWh}$

$10,821 \mathrm{kWh}$

1,237 kWh/kWp

$1,135 \mathrm{kWh} / \mathrm{kWp}$

$77.88 \%$
Losses - Gains (\%):

Shadings: $\quad-0.03$

Near mask : $\quad 0.00$

Partial shadings : $\quad 0.00$

IAM (reflection) : $\quad-2.75$

LID : $\quad 0.00$

Module dirt : $\quad-2.00$

Temperature : $\quad-1.77$

Module aging : $\quad-5.09$

Tolerance : $\quad 0.00$

Mismatch : $\quad-2.00$

DC cables : $\quad-0.50$

Inverter: $\quad-4.12$

Clipping : $\quad 0.00$

Power factor: $\quad 0.00$

AC cables : $\quad-0.31$

Unavailability : $\quad-2.00$

Monthly AC production (kWh/month):

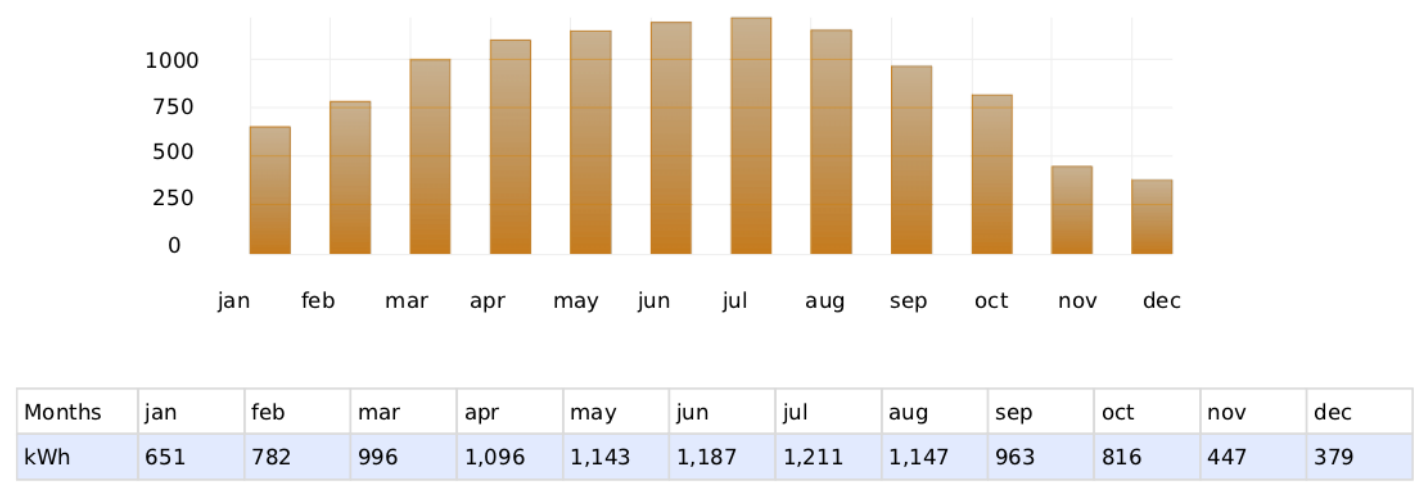




\section{archelios PRO archelios Pro study report (Version: 2020.1.01)}

四 Production (2)

Year per year AC production (kWh):

12000

10000

8000

6000

4000

2000

0
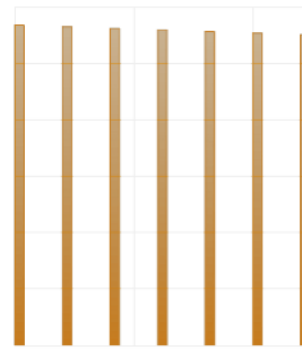

$2.5 \quad 5.0$
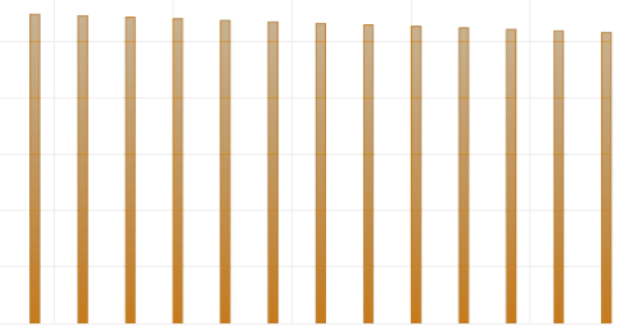

0.0

7.5

10.0

12.5

15.0

17.5

\begin{tabular}{|l|r|r|r|r|r|r|r|r|r|r|r|r|r|}
\hline Years & $\mathbf{1}$ & $\mathbf{2}$ & $\mathbf{3}$ & $\mathbf{4}$ & $\mathbf{5}$ & $\mathbf{6}$ & $\mathbf{7}$ & $\mathbf{8}$ & $\mathbf{9}$ & $\mathbf{1 0}$ \\
\hline $\mathrm{kWh}$ & 11,344 & 11,287 & 11,230 & 11,174 & 11,118 & 11,063 & 11,008 & 10,952 & 10,898 & 10,843 \\
\hline
\end{tabular}

\begin{tabular}{|l|r|r|r|r|r|r|r|r|r|r|r|r|}
\hline Years & $\mathbf{1 1}$ & $\mathbf{1 2}$ & $\mathbf{1 3}$ & $\mathbf{1 4}$ & $\mathbf{1 5}$ & $\mathbf{1 6}$ & $\mathbf{1 7}$ & $\mathbf{1 8}$ & $\mathbf{1 9}$ & $\mathbf{2 0}$ \\
\hline $\mathrm{kWh}$ & 10,789 & 10,735 & 10,681 & 10,628 & 10,575 & 10,522 & 10,469 & 10,417 & 10,365 & 10,313 \\
\hline
\end{tabular}

CARBON EQUIVALENT: 4 Carbon dioxide equivalent (tonne) *

* Quantity of greenhouse gases that would have been emitted on the observation period by producing this electricity by conventional means ( $20 \mathrm{~g}$ eq $\mathrm{CO} 2 / \mathrm{kWh}$ )

* Without considering the emissions of carbon generated by the manufacturing and the transporting of PV modules. 


\section{Glossary:}

Peak power:

Power (in Wp) delivered by the PV modules at Standard Test Conditions (STC)

(Standard Test Conditions : $1000 \mathrm{~W} / \mathrm{m}^{2}$, AM 1.5 spectrum, $25^{\circ} \mathrm{C}$ cell temperature)

Yield:

Energy produced by the photovoltaic installation, in kWh

$\mathrm{AC}$ :

Alternative Current

DC:

Direct Current

Specific Yield:

Yield normalized to installed power, in $\mathrm{kWh} / \mathrm{kWp}$

Performance Ratio:

ratio beetween specific yield and annual incident irradiation

Payback period:

ratio beetween annual cash-flow and investment

Expressed in years, it's the length of time required to recover the cost of the investment

Discount rate:

rate representing the cost of capital

NPV (Net Present Value):

Generated Value at the end of the observation period

Discounted payback period:

Observation time zeroing the net present value

Internal Rate of Return:

Discount rate nullifying the net present value

Profitability Index:

ratio beetween the net present present value and the investment

BOS, Balance Of System:

Cost including all components of a photovoltaic system, except the modules

LCOE (Levelized Cost Of Energy) or Overall discounted cost:

It is the ratio between the discounted cost of the project and the total energy produced over the observation period.

This is the cost per kWh produced by the installation (cost/kWh). 


\section{System Advisor Model Report}

Photovoltaic System

8.74 kW Nameplate

London A, ON

None

$43.03 \mathrm{~N},-81.15 \mathrm{E}$ GMT -5

\section{Performance Model}

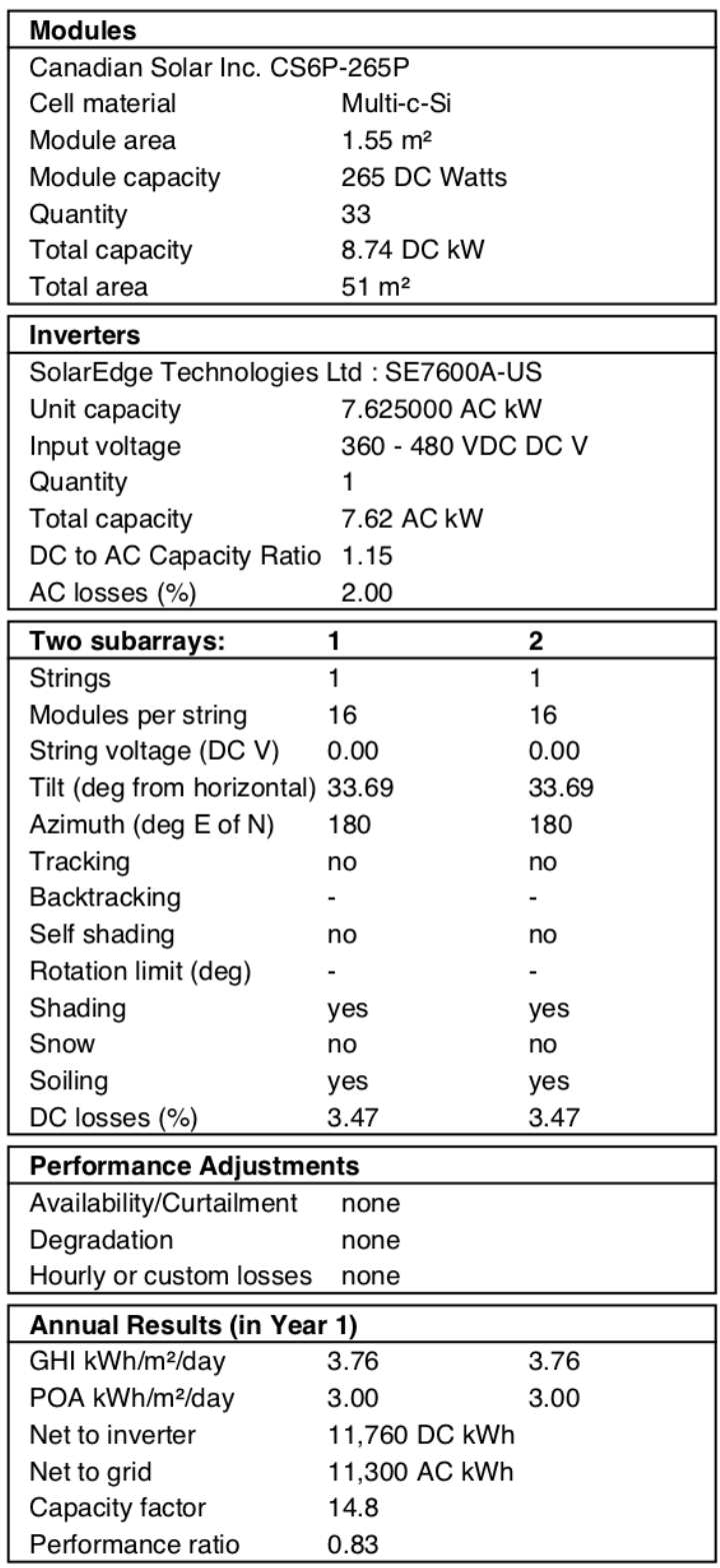

\section{No Financial model.}


Photovoltaic System

None
8.74 kW Nameplate

London $\mathrm{A}, \mathrm{ON}$

$43.03 \mathrm{~N},-81.15 \mathrm{E}$ GMT -5

No Financial model. 
Photovoltaic System

None

8.74 kW Nameplate

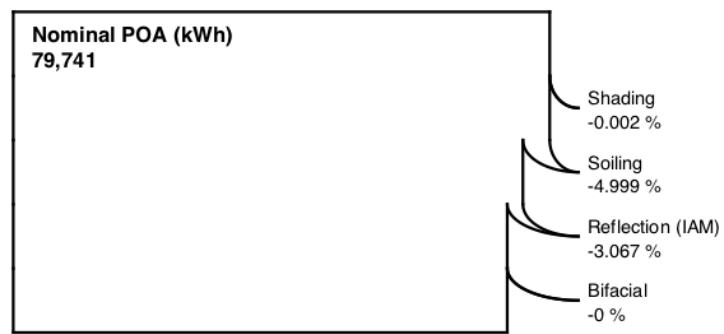

Nominal DC energy (kWh) 12,562

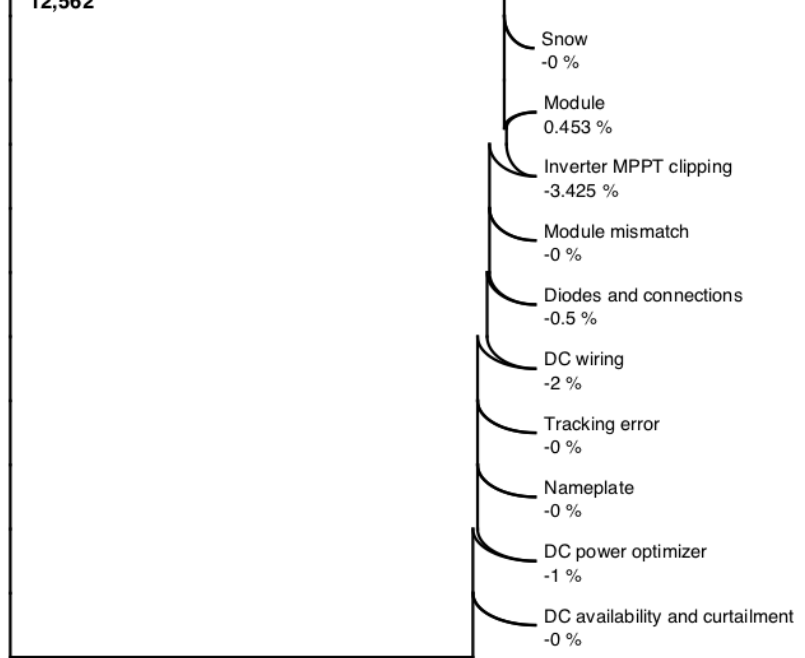

Net DC energy (kWh)

11,766

Inverter power clipping

$-0.071 \%$

Inverter power consumption

$-0.659 \%$

Inverter nighttime consumption

$-0.086 \%$

Inverter efficiency
$-2.148 \%$
London $\mathrm{A}, \mathrm{ON}$

43.03 N, -81.15 E GMT -5 


\section{REFERENCES}

Al-Homoud, M. S. (2001). Computer-aided building energy analysis techniques. Building and Environment, 36(4), 421-433.

Andrews, R., \& Yates, T. (2015). PV performance modeling assessing variability, uncertainty and sensitivity. Solarpro, 8.5, 20-38.

Axaopoulos, P. J., Fylladitakis, E. D., \& Gkarakis, K. (2014). Accuracy analysis of software for the estimation and planning of photovoltaic installations. International Journal of Energy and Environmental Engineering, 5(1), 1-7.

Bergamasco, L., \& Asinari, P. (2011). Scalable methodology for the photovoltaic solar energy potential assessment based on available roof surface area: Application to piedmont region (Italy). Solar Energy, 85(5), 1041-1055.

Berry, S., Whaley, D., Davidson, K., \& Saman, W. (2014). Do the numbers stack up? lessons from a zero carbon housing estate. Renewable Energy, 67(Complete), 80-89.

Blair, N., Dobos, A. P., Freeman, J., Neises, T., Wagner, M., Ferguson, T., Janzou, S. (2014). System advisor model (SAM) 2014.1.14: General description. (No. NREL/TP-6A2061019).

Blundell, J., \& Opitz, D. (2006). Object recognition and feature extraction from imagery: The feature analyst ${ }^{\circledR}$ approach. International Archives of Photogrammetry, Remote Sensing and Spatial Information Sciences, 36(4), C42.

Brown, S. J., \& Rowlands, I. H. (2009). Nodal pricing in Ontario, Canada: Implications for solar PV electricity. Renewable Energy, 34(1), 170-178.

Budischak, C., Sewell, D., Thomson, H., Mach, L., Veron, D. E., \& Kempton, W. (2013). Costminimized combinations of wind power, solar power and electrochemical storage, powering the grid up to $99.9 \%$ of the time. Journal of Power Sources, 225, 60-74. 
Caamano-Martin, E., Laukamp, H., Jantsch, M., Erge, T., Thornycroft, J., De Moor, H., .. . Gaiddon, B. (2008). Interaction between photovoltaic distributed generation and electricity networks. Progress in Photovoltaics: Research and Applications, 16, 629-643.

Cameron, C. P., Boyson, W. E., \& Riley, D. M. (2008). Comparison of PV system performancemodel predictions with measured PV system performance. Paper presented at the $200833 \mathrm{rd}$ IEEE Photovoltaic Specialists Conference, 1-6.

Cameron, C. P., Stein, J., \& Hansen, C. W. (2011). Evaluation of PV performance models and their impact on project risk. Paper presented at the PV Rollout Conference, Boston, MA.

CANMET Energy, Mines and Resources Canada. (1991). Photovoltaic systems design manual. Ottawa, Canada.

Carlisle, N., Elling, J., \& Penney, T. R. (2008). A renewable energy community: Key elements National Renewable Energy Laboratory.

Catita, C., Redweik, P., Pereira, J., \& Brito, M. (2014). Extending solar potential analysis in buildings to vertical facades. Computers \& Geosciences, 66, 1-12.

Chow, A., Li, S., \& Fung, A. (2016). Modeling urban solar energy with high spatiotemporal resolution: A case study in Toronto, Canada. International Journal of Green Energy, 13(11), 1090-1101.

Chow, A., Fung, A. S., \& Li, S. (2014). GIS modeling of solar neighborhood potential at a fine spatiotemporal resolution. Buildings, 4(2), 195-206.

Chow, T. T., Chan, A. L. S., Fong, K. F., \& Lin, Z. (2005). Hong Kong solar radiation on building facades evaluated by numerical models. Applied Thermal Engineering, 25(13), 1908-1921.

Chow, T. T., Hand, J. W., \& Strachan, P. A. (2003). Building-integrated photovoltaic and thermal applications in a subtropical hotel building. Applied Thermal Engineering, 23(16), 2035-2049. 
Compagnon, R. (2004). Solar and daylight availability in the urban fabric. Energy and Buildings, 36(4), 321-328.

Crawley, D. B., Hand, J. W., Kummert, M., \& Griffith, B. T. (2005). Contrasting the capabilities of building energy performance simulation programs. Washington, DC: US Department of Energy.

Crawley, D. B., Hand, J. W., Kummert, M., \& Griffith, B. T. (2008). Contrasting the capabilities of building energy performance simulation programs. Building and Environment, 43(4), 661-673.

Daneva, M. (1995). Software benchmark design and use. Re-engineering the enterprise (pp. 2029) Springer.

Dubayah, R., \& Rich, P. (1995). Topographic solar radiation models for GIS. International Journal of Geographical Information Systems, 9(4), 405-419.

Duffie, J. A., \& Beckman, W. A. (1991). Solar engineering of thermal processes (Second Edition ed.). New York: John Wiley \& Sons, Inc.

Duffie, J. A. (2013). In Beckman W. A. (Ed.), Solar engineering of thermal processes (4th ed. ed.). Hoboken, New Jersey: John Wiley \& Sons.

Eloranta, E., \& Crom, S. (1995). Performance indicators. Benchmarking —-theory and practice (pp. 391-396) Springer.

Environment Canada. (2008). In Meteorological Service of Canada and the National Research Council of Canada (Ed.), Canadian weather and engineering data sets (CWEEDS files) updated user's manual. Ottawa, Canada.

Erdélyi, R., Wang, Y., Guo, W., Hanna, E., \& Colantuono, G. (2014). Three-dimensional SOlar RAdiation model (SORAM) and its application to 3-D urban planning. Solar Energy, 101, 63-73. 
Esclapés, J., Ferreiro, I., Piera, J., \& Teller, J. (2014). A method to evaluate the adaptability of photovoltaic energy on urban façades. Solar Energy, 105, 414-427.

ESRI. ArcGIS Help 10.1. (2012a). Understanding solar radiation analysis. Retrieved from http://resources.arcgis.com/en/help/main/10.1/index.html\#//009z000000t8000000

ESRI. ArcGIS Help 10.1. (2012b). How solar radiation is calculated. 2012. Retrieved from http://resources.arcgis.com/en/help/main/10.1/index.html\#/How_solar_radiation_is_calculate d/00 9z000000tm000000/

ESRI. ArcGIS Desktop 10.2 Help. (2012c). Area solar radiation (Spatial Analyst). Retrieved from http://resources.arcgis.com/en/help/main/10.2/index.html\#//009z000000t5000000

ESRU. (no date). ESP-r, multi-platform building energy software tool. Retrieved from http://www.esru.strath.ac.uk/Programs/ESP-r_overview.htm

Folsom Labs. (2018). Shade modeling - HelioScope knowledge base. Retrieved from https://help.helioscope.com/article/54-shade-modeling

Freeman, J., \& Simon, J. (2015). Analysis of Aurora's performance simulation engine for three systems. NREL Technical Report NREL/TP-7A40-64213.

Freeman, J., Whitmore, J., Blair, N., \& Dobos, A. P. (2014). Validation of multiple tools for flat plate photovoltaic modeling against measured data. Paper presented at the 2014 IEEE 40th Photovoltaic Specialist Conference (PVSC), 1932-1937.

Freeman, J., Whitmore, J., Kaffine, L., Blair, N., \& Dobos, A. P. (2013). System Advisor Model: flat plate Photovoltaic performance modeling validation report. NREL Technical Report NREL/TP-6A20-60204.

Freitas, S., Catita, C., Redweik, P., \& Brito, M. (2015). Modelling solar potential in the urban environment: State-of-the-art review. Renewable and Sustainable Energy Reviews, 41, 915931. 
Fu, P., \& Rich, P. (1999). Design and implementation of the solar analyst: An ArcView extension for modeling solar radiation at landscape scales. Paper presented at the Proceedings of the Nineteenth Annual ESRI User Conference.

Green, M. A. (2004). Recent developments in photovoltaics. Solar Energy, 76(1-3), 3-8.

Grewe, V., Moussiopoulos, N., Builtjes, P., Borrego, C., Isaksen, I., \& Volz-Thomas, A. (2012). The ACCENT-protocol: A framework for benchmarking and model evaluation. Geoscientific Model Development, 5(3), 611-618.

Guittet, D. L., \& Freeman, J. M. (2018). Validation of photovoltaic modeling tool HelioScope against measured data. NREL Technical Report NREL/TP-6A20-72155.

Gurupira, T., \& Rix, A. (2017). PV simulation software comparisons: PVsyst, NREL SAM and pvlib. Paper presented at the Conf.: SAUPEC.

Hachem, C., Athienitis, A., \& Fazio, P. (2011). Solar optimized neighbourhood patterns: Evaluation and guide-lines. Proc. of eSim.

Hachem, C., Athienitis, A., \& Fazio, P. (2012). Evaluation of energy supply and demand in solar neighborhood. Energy \& Buildings, 49, 335-347.

Hay, J. E. (1979). Calculation of monthly mean solar radiation for horizontal and inclined surfaces. Solar Energy, 23(4), 301-307.

Hay, J. E., \& McKay, D. C. (1985). Estimating solar irradiance on inclined surfaces: A review and assessment of methodologies. International Journal of Solar Energy, 3(4-5), 203-240.

Hofierka, J., \& Suri, M. (2002). The solar radiation model for open source GIS: Implementation and applications. Paper presented at the Proceedings of the Open Source GIS - GRASS Users Conference, Italy.

Hofierka, J., \& Zlocha, M. (2012). A new 3-D solar radiation model for 3-D city models. Transactions in GIS, 16(5), 681-690. 
Holmgren, W., Hansen, C., \& Mikofski, M. (2018). Pvlib python: A python package for modeling solar energy systems. Journal of Open Source Software, 3(29), 884.

HOMER Energy. (2018). HOMER - hybrid renewable and distributed generation system design software. Retrieved from https://www.homerenergy.com/

Hren, R. (2015, Performance modeling tools overview. Solarpro, 8.5, 12-18.

Huppler, K. (2009). The art of building a good benchmark. Paper presented at the Technology Conference on Performance Evaluation and Benchmarking, 18-30.

Ineichen, P. (2011). Global irradiance on tilted and oriented planes: Model validations.

International Energy Agency (IEA). (2010). Technology roadmap - solar photovoltaic energy. Retrieved from http://www.iea.org/publications/freepublications/publication/pv_roadmap.pdf

International Energy Agency (IEA). (2012). Report T.41.B.3 - solar design of buildings for architects: Review of solar design tools. Retrieved from http://archive.ieashc.org/publications/downloads/T41B3_approved-Jul12.pdf

International Energy Agency (IEA). (2017). PV performance modeling methods and practices results from the 4th PV performance modeling collaborative workshop. (No. IEA-PVPS T13-06:2017).

ISO/IEC/IEEE 24765:2010. (2010). International standard ISO/IEC/IEEE 24765 systems and software engineering - vocabulary. Switzerland.

Izquierdo, S., Rodrigues, M., \& Fueyo, N. (2008). A method for estimating the geographical distribution of the available roof surface area for large-scale photovoltaic energy-potential evaluations. Solar Energy, 82(10), 929-939. 
Jakica, N. (2018). State-of-the-art review of solar design tools and methods for assessing daylighting and solar potential for building-integrated photovoltaics. Renewable and Sustainable Energy Reviews, 81, 1296-1328.

Jochem, A., Hofle, B., \& Rutzinger, M. (2011). Extraction of vertical walls from mobile laser scanning data for solar potential assessment. Remote Sensing, 3, 650-667.

Jochem, A., Höfle, B., Rutzinger, M., \& Pfeifer, N. (2009). Automatic roof plane detection and analysis in airborne lidar point clouds for solar potential assessment. Sensors, 9(7), 52415262.

Kalogirou, S. A. (2014). Solar energy engineering processes and sytems (Second edition ed.). United States of America: Academic Press.

Karteris, M., Theodoridou, I., Mallinis, G., \& Papadopoulos, A. M. (2014). Façade photovoltaic systems on multifamily buildings: An urban scale evaluation analysis using geographical information systems. Renewable and Sustainable Energy Reviews, 39(Complete), 912-933.

Kaygusuz, K. (2009). Environmental impacts of the solar energy systems. Energy Sources, Part A: Recovery, Utilization and Environmental Effects, 31, 1376-1386.

Kemery, B. P., Beausoleil-Morrison, I., \& Rowlands, I. H. (2012, May). Optimal PV orientation and geographic dispersion: a study of 10 Canadian cities and 16 Ontario locations. In Proceedings of the Canadian Conference on Building Simulation, Halifax, NS, Canada (pp. 1-4).

Klise, G. T., \& Stein, J. S. (2009). Models used to assess the performance of photovoltaic systems. Sandia National Laboratories. Technical Report SAND2009-8258.

Klucher, T. M. (1979). Evaluation of models to predict insolation on tilted surfaces. Solar Energy, 23(2), 111-114. 
Kucuksari, S., Khaleghi, A. M., Hamidi, M., Zhang, Y., Szidarovszky, F., Bayraksan, G., \& Son, Y. (2014). An integrated GIS, optimization and simulation framework for optimal PV size and location in campus area environments. Applied Energy, 113(Complete), 1601-1613.

Lalwani, M., Kothari, D., \& Singh, M. (2010). Investigation of solar photovoltaic simulation softwares. International Journal of Applied Engineering Research, 1(3), 586-601.

Lee, G. R., Frearson, L., \& Rodden, P. (2011). An assessment of photovoltaic modelling software using real world performance data. Paper presented at the 26th European International Conference on Photovoltaic Solar Energy, 4339-43.

Li, Z., Han, Y., \& Xu, P. (2014). Methods for benchmarking building energy consumption against its past or intended performance: An overview. Applied Energy, 124, 325-334.

Liu, B. Y. H., \& Jordan, R. C. (1960). The interrelationship and characteristic distribution of direct, diffuse and total solar radiation. Solar Energy, 4(3), 1-19.

Longley, P. A., Goodchild, M. F., Maguire, D. J., \& Rhind, D. W. (2005). Geographic information systems and science. John Wiley \& Sons.

Ma, T., Yang, H., \& Lu, L. (2014). Solar photovoltaic system modeling and performance prediction. Renewable and Sustainable Energy Reviews, 36, 304-315.

Machete, R., Falcão, A. P., Gomes, M. G., \& Rodrigues, A. M. (2018). The use of 3D GIS to analyse the influence of urban context on buildings' solar energy potential. Energy and Buildings, 177, 290-302.

Mainzer, K., Killinger, S., McKenna, R., \& Fichtner, W. (2017). Assessment of rooftop photovoltaic potentials at the urban level using publicly available geodata and image recognition techniques. Solar Energy, 155, 561-573.

Maneva, N., Daneva, M., \& Petrova, V. (1995). Benchmarking in software development. Benchmarking-Theory and practice (pp. 166-175) Springer. 
Mardaljevic, J., \& Rylatt, M. (2003). Irradiation mapping of complex urban environments: An image-based approach. Energy and Buildings, 35(1), 27-35.

Marsh, A. (2003). Ecotect and EnergyPlus. Building Energy Simulation User News, 24(6).

McKenney, D. W., Pelland, S., Poissant, Y., Morris, R., Hutchinson, M., Papadopol, P., Campbell, K. (2008). Spatial insolation models for photovoltaic energy in Canada. Solar Energy, 82(11), 1049-1061.

Merriam-Webster. (2014). Benchmark. Retrieved from http://www.merriamwebster.com/dictionary/benchmark

Meteonorm. (2019). Meteonorm intro. Retrieved from https://meteonorm.com/en

Miguel, J. P., Mauricio, D., \& Rodríguez, G. (2014). A review of software quality models for the evaluation of software products. ArXiv Preprint arXiv:1412.297.

Morris, R. (2016). Final report - updating CWEEDS weather files. ( No. EC contract 3000607888).

Muneer, T., Kambezidis, H., \& Gueymard, C. (2004). Solar radiation and daylight models: With software available from companion web site (2nd ed.) Elsevier Butterworth Heinemann.

Nambiar, R., Poess, M., \& Transaction Processing Performance Council. (2009). Performance evaluation and benchmarking: First TPC technology conference, TPCTC 2009, lyon, france, august 24-28, 2009 : Revised selected papers Springer.

National Renewable Energy Laboratory (NREL). (2020). PVWatts calculator. Retrieved from https://pvwatts.nrel.gov/

Natural Resources Canada (NRC). (2002). Photovoltaic systems a buyer's guide. Ottawa: Natural Resources Canada. 
Natural Resources Canada (NRCan). (2019). RETScreen natural resources Canada. Retrieved from https://www.nrcan.gc.ca/maps-tools-publications/tools/data-analysis-softwaremodelling/retscreen/7465

Nguyen, H. T., \& Pearce, J. M. (2012). Incorporating shading losses in solar photovoltaic potential assessment at the municipal scale. Solar Energy, 86(5), 1245-1260.

Nguyen, H. T., Pearce, J. M., Harrap, R., \& Barber, G. (2012). The application of LiDAR to assessment of rooftop solar photovoltaic deployment potential in a municipal district unit. Sensors, 12, 4534-4558.

Nouvel, R., Zirak, M., Dastageeri, H., Coors, V., \& Eicker, U. (2014). Urban energy analysis based on $3 \mathrm{~d}$ city model for national scale applications. Paper presented at the Presented at the IBPSA Germany Conference,.

O'Bnen, W. T., Kennedy, C. A., Athienitis, A. K., \& Kesik, T. J. (2010). The relationship between net energy use and the urban density of solar buildings. Environment \& Planning B: Planning \& Design, 37(6), 1002-1021.

Oxford Dictionary. (2014). Benchmark. Retrieved from http://www.oxforddictionaries.com/definition/english/benchmark

Pandey, C. K., \& Katiyar, A. K. (2009). A note on diffuse solar radiation on a tilted surface. Energy, 34(11), 1764-1769.

Parida, B., Iniyan, S., \& Goic, R. (2011). A review of solar photovoltaic technologies. Renewable and Sustainable Energy Reviews, 15(3), 1625-1636.

Perez, R., Ineichen, P., Seals, R., Michalsky, J., \& Stewart, R. (1990). Modeling daylight availability and irradiance components from direct and global irradiance. Solar Energy, 44(5), 271-289.

Perez, R., Seals, R., Ineichen, P., Stewart, R., \& Menicucci, D. (1987). A new simplified version of the Perez diffuse irradiance model for tilted surfaces. Solar Energy, 39(3), 221-231. 
Perez, R., \& Stewart, R. (1986). Solar irradiance conversion models. Solar Cells, 18(3-4), 213222.

PVGIS. (2020). Photovoltaic geographic information system (PVGIS) EU science hub. Retrieved from https://ec.europa.eu/jrc/en/pvgis

PVsyst. (2019). PVsyst 6 help - transposition models. Retrieved from https://www.PVsyst.com/help/

PVsyst. (2020). PVsyst - logiciel photovoltaique. Retrieved from https://www.PVsyst.com/

Ramirez-Rosado, I. J., Fernandez-Jimenez, L. A., Monteiro, C., Garcia-Garrido, E., \& ZorzanoSantamaria, P. (2011). Spatial long-term forecasting of small power photovoltaic systems expansion. Renewable Energy, 36(12), 3499-3506.

Redweik, P., Catita, C., \& Brito, M. (2013). Solar energy potential on roofs and facades in an urban landscape. Solar Energy, 97, 332-341.

Reindl, D., Beckman, W., \& Duffie, J. (1990). Evaluation of hourly tilted surface radiation models. Solar Energy, 45(1), 9-17.

Resch, B., Sagl, G., Törnros, T., Bachmaier, A., Eggers, J., Herkel, S., Gündra, H. (2014). GISbased planning and modeling for renewable energy: Challenges and future research avenues. ISPRS International Journal of Geo-Information, 3(2), 662-692.

Rich, P., Dubayah, R., Hetrick, W., \& Saving, S. (1994). Using viewshed models to calculate intercepted solar radiation: Applications in ecology. American society for photogrammetry and remote sensing technical papers. Paper presented at the American Society of Photogrammetry and Remote Sensing, 524-529.

Roberts, J. J., Mendiburu Zevallos, A. A., \& Cassula, A. M. (2017). Assessment of photovoltaic performance models for system simulation. Renewable and Sustainable Energy Reviews, 72, 1104-1123. 
Robinson, D. (2006). Urban morphology and indicators of radiation availability. Solar Energy, 80(12), 1643-1648.

Robinson, D., Haldi, F., Kämpf, J., Leroux, P., Perez, D., Rasheed, A., \& Wilke, U. (2009). CitySim: Comprehensive micro-simulation of resource flows for sustainable urban planning. Paper presented at the Proc. Building Simulation.

Robinson, D., Campbell, N., Gaiser, W., Kabel, K., Le-Mouel, A., Morel, N., Stone, A. (2007). SUNtool - A new modelling paradigm for simulating and optimising urban sustainability. Solar Energy, 81(9), 1196-1211.

Rolstadås, A. (1995). Benchmarking—theory and practice Springer.

Romero Rodríguez, L., Duminil, E., Sánchez Ramos, J., \& Eicker, U. (2017a). Assessment of the photovoltaic potential at urban level based on 3D city models: A case study and new methodological approach. Solar Energy, 146, 264-275.

Romero Rodríguez, L., Nouvel, R., Duminil, E., \& Eicker, U. (2017b). Setting intelligent city tiling strategies for urban shading simulations. Solar Energy, 157, 880-894.

Ruiz-Arias, J. A., Tovar-Pescador, J., Pozo-Vázquez, D., \& Alsamamra, H. (2009). A comparative analysis of DEM-based models to estimate the solar radiation in mountainous terrain. International Journal of Geographical Information Science, 23(8), 1049-1076.

Rylatt, M., Gadsden, S., \& Lomas, K. (2001). GIS-based decision support for solar energy planning in urban environments. Computers, Environment and Urban Systems, 25(6), 579603.

SAM NREL. (2020). Home- system advisor model (SAM). Retrieved from https://sam.nrel.gov/

Santos, T., Gomes, N., Freire, S., Brito, M. C., Santos, L., \& Tenedório, J. A. (2014). Applications of solar mapping in the urban environment. Applied Geography, 51(Complete), 48-57. 
Sarralde, J. J., Quinn, D. J., Wiesmann, D., \& Steemers, K. (2015). Solar energy and urban morphology: Scenarios for increasing the renewable energy potential of neighbourhoods in london. Renewable Energy, 73(Complete), 10-17.

Sharma, D. K., Verma, V., \& Singh, A. P. (2014). Review and analysis of solar photovoltaic softwares. International Journal of Current Engineering and Technology, 4(2), 725-731.

Sim, S. E., Easterbrook, S., \& Holt, R. C. (2003). Using benchmarking to advance research: A challenge to software engineering. Paper presented at the Proceedings of the 25th International Conference on Software Engineering, 74-83.

Skelion. (no date). Skelion featues - sketchup skelion solar design plugin renewable energy. Retrieved from http://skelion.com/en/features.htm?v1.0.0

Stein, J. S., Cameron, C. P., Bourne, B., Kimber, A., Posbic, J., \& Jester, T. (2010, June). A standardized approach to PV system performance model validation. In 201035 th IEEE Photovoltaic Specialists Conference (pp. 001079-001084). IEEE.

St. Denis, G., \& Parker, P. (2009). Community energy planning in Canada: The role of renewable energy. Renewable and Sustainable Energy Reviews, 13(8), 2088-2095.

Strzalka, A., Alam, N., Duminil, E., Coors, V., \& Eicker, U. (2012). Large scale integration of photovoltaics in cities. Applied Energy, 93, 413-421.

Takebayashi, H., Ishii, E., Moriyama, M., Sakaki, A., Nakajima, S., \& Ueda, H. (2015). Study to examine the potential for solar energy utilization based on the relationship between urban morphology and solar radiation gain on building rooftops and wall surfaces. Solar Energy, $119,362-369$.

Teller, J., \& Azar, S. (2001). Townscope II-A computer system to support solar access decision-making. Solar Energy, 70(3), 187-200.

Temps, R. C., \& Coulson, K. (1977). Solar radiation incident upon slopes of different orientations. Solar Energy, 19(2), 179-184. 
Tereci, A., Ozkan, S. T. E., \& Eicker, U. (2013). Energy benchmarking for residential buildings. Energy and Buildings, 60, 92-99.

Tichy, W. F. (1998). Should computer scientists experiment more? Computer, 31(5), 32-40.

TRNSYS. (2019). Welcome TRNSYS transient system simulation tool. Retrieved from http://www.trnsys.com/

Tsoutsos, T., Frantzeskaki, N., \& Gekas, V. (2005). Environmental impacts from the solar energy technologies. Energy Policy, 33(3), 289-296.

Tyagi, V. V., Rahim, N. A. A., Rahim, N. A., \& Selvaraj, J. A. L. (2013). Progress in solar PV technology: Research and achievement. Renewable and Sustainable Energy Reviews, 20, 443-461.

U.S. Department of Energy (DOE). (2013). EnergyPlus energy simulation software. Retrieved from http://apps1.eere.energy.gov/buildings/energyplus/weatherdata_about.cfm

Ukovich, W., \& Zerilli, F. (1995). Modelling techniques for benchmarking in complex administration systems: An approach from a total quality viewpoint. Benchmarking-Theory and practice (pp. 210-219) Springer.

Vieira, M., \& Madeira, H. (2009). From performance to dependability benchmarking: A mandatory path. Paper presented at the Technology Conference on Performance Evaluation and Benchmarking, 67-83.

Voorhees, E. M. (2005). TREC: Improving information access through evaluation. Bulletin of the American Society for Information Science and Technology, 32(1), 16-21.

Wiginton, L. K., Nguyen, H. T., \& Pearce, J. M. (2010). Quantifying rooftop solar photovoltaic potential for regional renewable energy policy. Computers, Environment and Urban Systems, 34(4), 345-357. 
Wilcox, S., \& Marion, W. (2008). Users manual for TMY3 data sets National Renewable Energy Laboratory Golden, CO.

Yates, T., \& Hibberd, B. (2010) Production modeling for grid-tied PV systems. Solarpro, Issue 3.3 Apr/May, 30-56. 